\title{
USING MOBILE APPLICATIONS IN CAREGIVING: THE USER EXPERIENCES OF FAMILY CAREGIVERS OF PERSONS LIVING WITH DEMENTIA
}

\author{
By \\ Angel He Wang \\ BScN, 2015 \\ Ryerson University, Toronto, Canada \\ A thesis \\ presented to Ryerson University \\ in partial fulfillment of the \\ requirements for the degree of \\ Master of Nursing \\ in the Program of \\ Nursing
}

Toronto, Ontario, Canada, 2019

(C) Angel He Wang, 2019 


\section{AUTHOR'S DECLARATION FOR ELECTRONIC SUBMISSION OF A THESIS}

I hereby declare that I am the sole author of this thesis. This is a true copy of the thesis, including any required final revisions, as accepted by my examiners.

I authorize Ryerson University to lend this thesis to other institutions or individuals for the purpose of scholarly research.

I further authorize Ryerson University to reproduce this thesis by photocopying or by other means, in total or in part, at the request of other institutions or individuals for the purpose of scholarly research.

I understand that my thesis may be made electronically available to the public. 


\title{
ABSTRACT \\ USING MOBILE APPLICATIONS IN CAREGIVING: THE USER EXPERIENCES OF FAMILY CAREGIVERS OF PERSONS LIVING WITH DEMENTIA
}

\author{
By \\ Angel He Wang \\ Master of Nursing, 2019 \\ Master of Nursing Degree Program \\ Daphne Cockwell School of Nursing, Ryerson University, Toronto
}

To date, there is no research that explores the user experiences of family caregivers of persons living with dementia on using mobile applications to support caregiving activities. This is of particular concern given that limited understandings of the user experience in designing technology have often led to end-users experiencing barriers in technology adoption and use. Thus, the purpose of this qualitative descriptive study that used photo-elicitation interviewing was to explore the experiences of family caregivers of persons living with dementia on using mobile applications in their caregiving roles. The findings revealed how mobile applications played an important role in the lives of the caregiver, the care recipient, and both together as a dyad. This research advances our understanding of the impact of mobile application use in caregiving and provides direction for future research, policy, education, practice and application development. 


\section{ACKNOWLEDGEMENTS}

My thesis journey would have not been possible without the support of many people, to whom I am deeply grateful for. To my co-supervisors, Dr. Kristine Newman and Dr. Lori Schindel Martin, thank you for pushing me to expand my way of thinking in ways I never thought was possible. My sincere gratitude and appreciation for all that you have done especially all the knowledge and wisdom you have imparted on me. I would also like to thank my committee member, Dr. Jennifer Lapum for her continued support and dedication over the years. Thank you for your kind words, your attention to detail which inspired me to consider the small, yet significant, aspects of the research process, and most importantly, your encouragement for me to be creative. A heartfelt thank you to my thesis committee as this whole journey made me grow not only as a researcher, but also as a person.

My heartfelt gratitude towards all the wonderful faculty at Ryerson University whom I had the opportunity to have been in their classes or work with: Dr. Suzanne Fredericks, Dr. Sherry Espin, Dr. Heather Beanlands, Dr. Jasna Schwind (*heart hugs!*), Dr. Elizabeth McCay, Dr. Cristina Catallo, and Dr. Coralee McLaren. A special thank you to Dr. Oona St-Amant for introducing me to the world of research as she was the first professor that offered me a research assistant position. This important opportunity ultimately cultivated my passion for nursing research. Another very special thank you to Dr. Charlotte Lee for graciously taking me under your wings and showing me how to be an amazing educator during my $\mathrm{MN}$ practicum. It meant the world to me when you gifted me my first recorder for my thesis research. You have not only been a fantastic mentor, but also a great friend. 
I would like to express my deepest gratitude to the participants in this study. Thank you for sharing your time and experiences with me as they have had a lasting impact on me. Thank you for participating in the study with such passion, courage, and enthusiasm.

A special thank you to all the individuals and organizations who helped me spread the word about this research. I sincerely appreciate all the support that you have provided.

I would like to acknowledge and thank the Graduate Studies at Ryerson University, Alzheimer's Society Research Program, Canadian Nurses Foundation, Nursing Leadership Network of Ontario, and Canadian Gerontological Nursing Association for their financial support of this research project.

Lastly, and most importantly, thank you to my friends and family for their unwavering support, patience, and love during this whole journey. Thank you for cheering me on from the sidelines, especially when it was the toughest. 


\section{DEDICATION}

To all the caregivers: thank you for all that you do.

And 我亲爱的母亲 (my beloved mother). 


\section{TABLE OF CONTENTS}

\begin{tabular}{|c|c|}
\hline & Page \\
\hline AUTHOR'S DECLARATION & ii \\
\hline 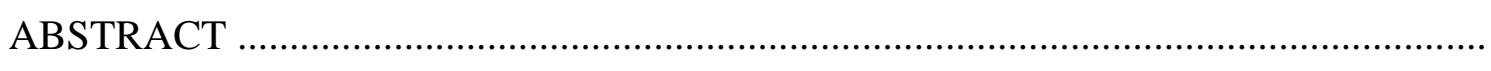 & iii \\
\hline ACKNOWLEDGEMENTS & iv \\
\hline DEDICATION . & vi \\
\hline LIST OF TABLES ................................. & $\mathrm{x}$ \\
\hline LIST OF FIGURES . & $\mathrm{xi}$ \\
\hline LIST OF PHOTOGRAPHS . & xii \\
\hline LIST OF ABBREVIATIONS . & xiii \\
\hline GLOSSARY OF SIGNIFICANT TERMS & xiv \\
\hline LIST OF APPENDICES . & $\mathrm{XV}$ \\
\hline PROLOGUE: MY EXPERIENCES OF CAREGIVING ...................... & 1 \\
\hline CHAPTER ONE: INTRODUCTION ............................... & 8 \\
\hline PART I: BACKGROUND ........................... & 9 \\
\hline 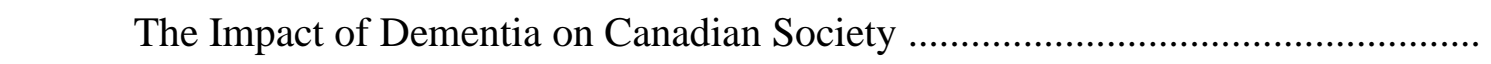 & 10 \\
\hline 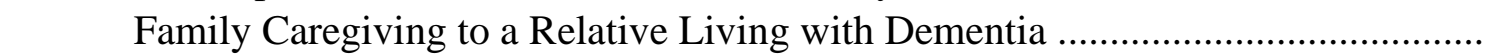 & 12 \\
\hline How Mobile Technologies Have Influences Family Caregiving ........................... & 14 \\
\hline PART II: SITUATING THE STUDY ................. & 18 \\
\hline 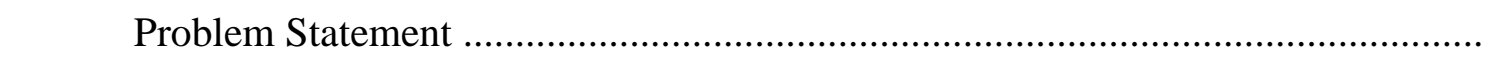 & 18 \\
\hline 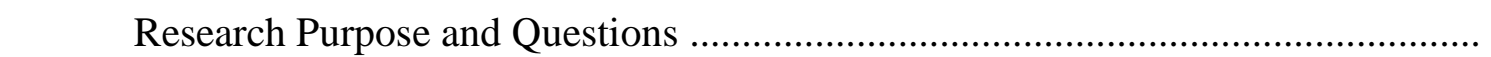 & 19 \\
\hline 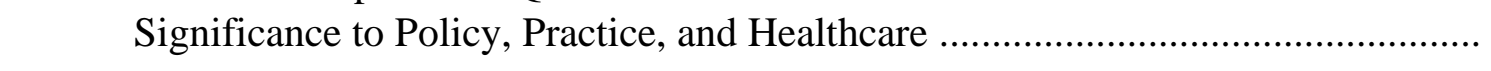 & 20 \\
\hline CHAPTER TWO: PHILOSOPHICAL UNDERPINNINGS .. & 22 \\
\hline 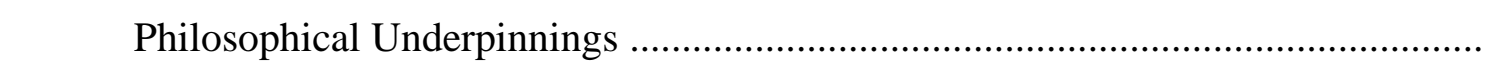 & 23 \\
\hline Situated Philosophical Foundation ............. & 24 \\
\hline The Constructivism Paradigm & 24 \\
\hline Philosophical Assumptions ... & 25 \\
\hline
\end{tabular}

\section{CHAPTER THREE: REVIEW AND SYNTHESIS OF RELEVANT}

LITERATURE

Literature Search Strategy ..................................................................... 31

Literature Review and Synthesis .............................................................. 33 
Background: Family Caregiving

Part I: Experiences of Family Caregivers of Persons Living with

Dementia

Part II: Technology and Caregiving .....

Part III: Understanding the User Experience in the Dementia Context ..... 53

\section{CHAPTER FOUR: RESEARCH METHODOLOGY, STUDY METHODS AND RESEARCH PROCESS}

PART I: RESEARCH METHODOLOGY

Qualitative Description

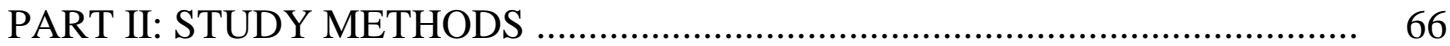

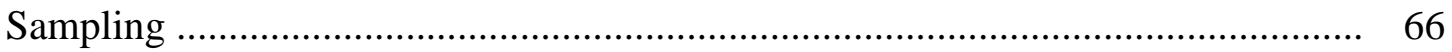

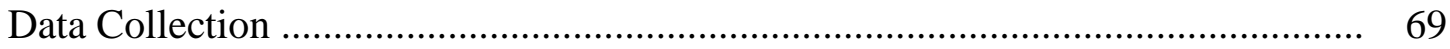

Data Analysis: Thematic Analysis ............................................................... 79

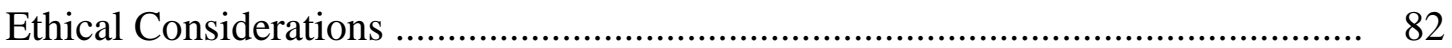

PART III: REFLEXIVITY AND TRUSTWORTHINESS .............................. 83

Reflexivity ................................................................................. 83

Trustworthiness: Establishing Rigour in Qualitative Research ........................... 84

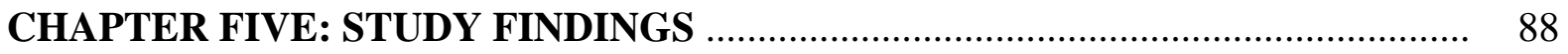

THE PARTICIPANTS …................................................................. 90

PART I: USER EXPERIENCES OF FAMILY CAREGIVERS ......................... 94

Overarching Theme: Connecting to Support Through Mobile Apps in My, Your,

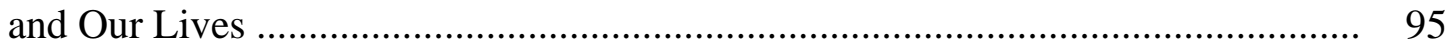

Main Theme \#1: Adapting Mobile Apps to Meet Individual Needs of the Dyad ... 96

Subtheme \#1: Connecting with Others ............................................... 97

Subtheme \#2: Assisting with Care Activities .......................................... 105

Subtheme \#3: Organizing Everyday Living ........................................... 111

Main Theme \#2: Minimizing the Impact of the Condition on the Person and the Family

Subtheme \#1: Promoting the Care Recipient's Personhood ..................... 117

Subtheme \#2: Maintaining Family Routines and Traditions ..................... 123

Main Theme \#3: Determining the Effectiveness of Mobile Apps ....................... 127

Subtheme \#1: Identifying Useful Functions of Mobile Apps ................... 127

Subtheme \#2: Envisioning the Potential of Mobile Apps ......................... 132

PART II: PARTICIPANTS’ EXPERIENCES WITH PHOTO-ELICITATION

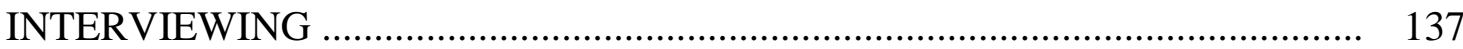

Main Theme: Promoted Reflection and Richer Dialogue ................................ 137 
CHAPTER SIX: DISCUSSION, LIMITATIONS, AND IMPLICATIONS ……….... 146

PART I: MOBILE APP USER EXPERIENCES OF FAMILY CAREGIVERS ... 148

Adapting Mobile Apps to Meet Individual Needs of the Dyad .............................. 149

Minimizing the Impact of the Condition on the Person and the Family ................ 158

Determining the Effectiveness of Mobile Apps ……………………………...... 168

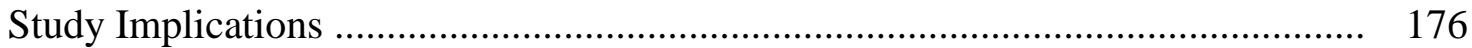

PART II: PARTICIPANTS' EXPERIENCE WITH PHOTO-ELICITATION 182 INTERVIEWING (PEI) .

Implications for Research ............................................................................ 185

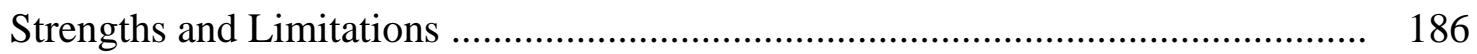

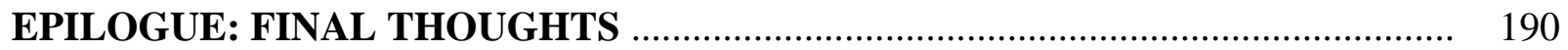

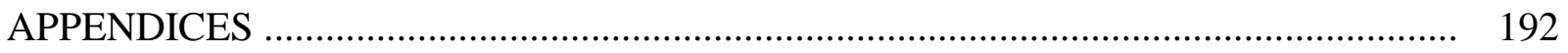

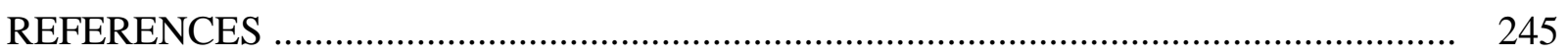




\section{LIST OF TABLES}

Page

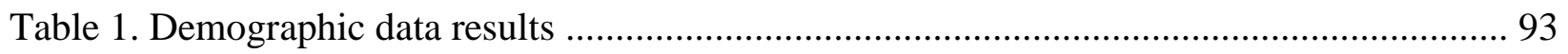




\section{LIST OF FIGURES}

Page

Figure 1. Overview of the interview data collection process employed in this study ............... 70

Figure 2. The photo-elicitation interviewing process for this study ...................................... 74

Figure 3. Visual representation of the study findings .................................................... 95 


\section{LIST OF PHOTOGRAPHS}

Page

Photo 1. "Technology allows me to be still connected to my family and lessen that gap between here and there"

Photo 2. "Staying connected"

Photo 3. "Social media keeps you connected to other people; they don't have to walk in your shoes because they are already walking in ones that are similar to yours".....

Photo 4. "Using a reminder to keep you in check of what has to be done, not just for you but for someone else"

Photo 5. "I am sitting somewhere else and I can take care of him sitting really far away"

Photo 6. "I end up spending a lot more time necessary when I could just be at home and spend more time with my family"

Photo 7. "That's how I kept organized - it was the only way I can mash up my personal life, my work life, and with having to care for grandparents"

Photo 8. "A mother's love"

Photo 9. "For her, everything goes around music"

Photo 10. "You can still bring some new experiences for him, for the person, just with technology"

Photo 11. "I was using mobile technology like Netflix... We wanted him to stay engaged"

Photo 12. "He wanted to get me something for my birthday; he ordered it on the app himself; and I was proud of him"

Photo 13. "We can spend time as a family and enjoy; rather than slaving two days before the actual event"

Photo 14. "She loves to have that kind of togetherness and the memories"

Photo 15. "Integration is really important especially in caregiving so you can stay connected to what you were doing no matter what device you are using"

Photo 16. "I would wish there could be an app to know when she is getting off the bus so that we're sure she is there, and the bus didn't make our lives harder"

Photo 17. "Pictures represent memories and I blurred it; I just see myself kind of in her shoes" 


\section{LIST OF ABBREVIATIONS}

$\begin{array}{ll}\text { ADLs } & \text { Activities of daily living } \\ \text { BPSD } & \text { Behavioural and psychological symptoms of dementia } \\ \text { IADLs } & \text { Instrumental activities of daily living } \\ \text { mHealth } & \text { Mobile health } \\ \text { PDAs } & \text { Personal digital assistants } \\ \text { PEI } & \text { Photo-elicitation interviewing } \\ \text { PICO } & \text { Population, Intervention, Comparison, Outcome } \\ \text { PICo } & \text { Population, Phenomena of Interest, Context } \\ \text { SMS } & \text { Short message service } \\ \text { TAM } & \text { Technology Acceptance Model } \\ \text { TTC } & \text { Toronto Transit Commission } \\ \text { UTAUT } & \text { Unified Theory of Acceptance and Use of Technology } \\ \text { WHO } & \text { World Health Organization }\end{array}$




\section{GLOSSARY OF SIGNIFICANT TERMS}

Family Caregiver In the context of this study, a family caregiver was defined as "an individual who is providing care, without pay or wage, to a family member in the following four domains: (1) care management; (2) assistance with instrumental activities of daily living; (3) assistance with activities of daily living; and/or (4) emotional and social support." Depending on life circumstances and the condition of the care recipient, family caregivers can range from providing one to four of the care provision categories.

Health-Related Mobile (mHealth) Apps

Mobile Apps

Usability

User Experience
mHealth apps is a category of mobile apps that focus on the provision of medical and public health services (WHO, 2011).

Mobile apps are software programs developed to run on mobile devices, such as smartphones, tablets, and laptops, which provide specific services for the user (Merriam-Webster, n.d.).

For the purposes of this study, the definition of usability provided by the International Organization for Standarization (ISO; 2018) was used: "the ability to use a product with effectiveness, efficiency, and satisfaction in a specific context of use."

The definition for user experience put forth by International Organization for Standardization (ISO; 2010) is the most standardized in the literature and thus, have been adapted for the purposes of this study. User experience is defined as "a person's perceptions and responses that result from the use a product, system or service." 


\section{LIST OF APPENDICES}

Page

APPENDIX A: Mobile Apps in the Dementia Context

APPENDIX B: Literature Review Framework

APPENDIX C: Table of Inclusion and Exclusion Criteria Used for Literature Review

Search

APPENDIX D: PRISMA Flow Diagram ………………….................................... 208

APPENDIX E: Literature Review References ……………………............................. 209

APPENDIX F: Recruitment Documents .................................................................. 223

Recruitment Email ...................................................................................... 223

Email Recruitment Message …………...................................................... 224

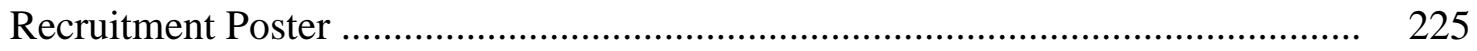

Recruitment Posts on Social Media Platforms ………....................................... 226

APPENDIX G: Telephone Screening Script ……………........................................... 228

APPENDIX H: Consent Agreement …………………….................................... 230

APPENDIX I: Demographic Data Form ……………..................................................... 237

APPENDIX J: First Interview - Interview Guide …………………………............... 238

APPENDIX K: Photography Activity - Information Handout ......................................... 240

APPENDIX L: Second Interview - Interview Guide ………………………………..... 241

APPENDIX M: List of Mobile Apps Used by Participants ................................................. 243 


\section{PROLOGUE}

\section{MY EXPERIENCES OF CAREGIVING}




\section{"Healing yourself is connected with healing others."}

\section{- Yoko Ono}

As I began navigating my thesis ideas, I took some time to reflect on my previous experiences, my beliefs, and particularly, what was important to me. This quote by Yoko Ono persistently surfaced in my reflections as it has been my philosophy and way of thinking since I was young when I first started my caregiving journey. In this prologue, I reflect on my experiences in caregiving when I took on three identities: family caregiver, formal caregiver, and care recipient. These experiences cultivated my passion in conducting research in caregiving, and ultimately led to my thesis study.

\section{My Experiences as a Family Caregiver}

My first family caregiving experience started when I was a teenager and this role continues to this day. I have provided care for loved ones who were experiencing mental and physical conditions, including major depressive and anxiety disorder, diabetes, heart conditions, dementia and final stages of cancer. While I reserve going in-depth with the details of all my caregiving experiences out of respect for the privacy of my family members, I do want to share briefly my story with Lily (a pseudonym). When Lily fell at home and had to be taken to the emergency department, my family and I were informed of the devastating diagnosis of stage four cancer. "We will continue to do more tests and make sure we manage her pain appropriately," Lily's oncologist asserted. While not perfect, I was incredibly grateful for the Canadian healthcare system from diagnosis to palliative care. The oncology care Lily received for months in the hospital as well as the palliative care she received at home in her final days was filled with respect, dignity, and compassion. 
However, as a family caregiver, I often felt silent, invisible, and unacknowledged as a caregiver as I was never asked or consulted about the nature and impact of my caregiving role. This was particularly salient when I was a young caregiver as I received limited professional assistance and was left to provide loved ones with intimate physical and emotional support that was demanding and challenging. Furthermore, my experiences showed me that caregiving is neither linear nor static, but rather, fluid and free-flowing. There are ups, downs, and everything in between. The quote by Yoko Ono elucidates the interdependence between the caregiver and care recipient in a way that strongly resonated with my experiences and my ways of making meaning in life. As the need for not only compassion for others, but also compassion for self, became more evident in my caregiving journey, I adopted a philosophy that emphasized the reciprocity within caregiving: what we want to heal in others, will in the end be a source of healing within ourselves. This philosophy, which blossomed out of my family caregiving experiences, ultimately led me to nursing.

\section{My Experiences as a Formal Caregiver}

After working as a Registered Nurse $(\mathrm{RN})$ for over four years, I have encountered a variety of patients who are unique in their own ways - making our experience together one of a kind. Although the general understanding is that nurses are givers while patients are the receivers, my perception differs as I view caregiving as a reciprocal process. While my experiences as a formal caregiver is wide-ranging given my time in a variety of nursing specialities, four encounters stand out that illuminate that concept of reciprocity in caregiving.

“Will you be in tomorrow?" is a question that I am often asked by patients and one that I always cherish no matter how many times I hear it. At times, the answer is yes and the patients' faces light up, and at times, I reluctantly whisper a no and the patients react in disappointment. 
Even though it is subtle and seen as a small gesture, it is what makes my work as a nurse worthwhile. Yes, the cards, coffee, cookies, and other treats that patients give to the healthcare team as a token of appreciation is always touching, but this question is what hits me the hardest. Always. It is a delicate validation that what I am doing matters and is making a small difference during their most vulnerable time.

The importance of holistic care was a concept that was heavily emphasized throughout my nursing education, but the following experience truly made me understand. I had just arrived on my shift and noticed my patient, who was living with dementia, in two-point soft restraints. "He was pulling out his intravenous lines and indwelling catheter as well as trying to hit us as we were trying to clean him, so we received orders from the doctor to restrain him", the Charge Nurse explained. Throughout the morning, he remained restless and vigilant. Then, a conversation I had with my patient's granddaughter surfaced in my mind. I remember her saying: "My grandfather really loves classical music, I always play it for him when I visit him in the nursing home." I knew that keeping him in restraints could lead to negative consequences and thought that I would try playing him some classical music from my phone to help manage his responsive behaviours. Instantly, I noticed a drastic change in behaviour as he began to focus and bask in the music. Afterwards, I removed the restraints and gave the granddaughter a call to give her an update on her grandfather's status. She was ecstatic that he was no longer in restraints and offered to bring in her iPod which had a playlist of music he likes as well as an audio-book of his favourite novel to keep by his bedside. A biomedical focus often predominates in acute care, but in this occurrence, I was reminded of the significance of considering the psychological, social, and cultural needs of a patient and its impact on patient outcomes. 
The intimacy of providing care is something I experience regularly as a nurse, but it was the following occurrence that put a spotlight on this form of intimacy. I was taking care of a patient who was quadriplegic (paralysis of all four limbs). Just as my evening shift was ending, he called to notify me that he was incontinent. "I am so sorry, I know you are done your shift. This is so embarrassing ... the whole bed is wet ... I hate burdening you guys to help me with everything", the patient said somberly. "It is okay! Don't worry about it. That is what I am here for. I will be right back with all the bedsheets and a brief, and I will change you", I said trying to reassure him. "Wait ... no ... don't. Just change my brief. That's too much work for you. You should go, it's late", my patient exclaimed. As I was changing the bedding and his brief, I could sense his feelings of embarrassment and helplessness - something this patient population unfortunately experiences regularly. I continued to reassure him that he is not a burden and tried to re-frame his way of thinking. As I was leaving, he softly whispered "Sorry that all I can do for you is say thank you." I remember trying not to get too emotional in front of him, and said "That's more than enough."

Behind the label of "drug addict" was an addictions counsellor. As I was getting morning report, the charge nurse stated: "Patient has been admitted with congestive heart failure and he is a previous drug addict ... he used a variety of illegal substances, you can find it in his chart.” This was my first time assigned to this patient. Although it was an acute care setting where majority of the time I am up and running around leaving minimal amount of time to really sit and talk with patients, that day was different. I had some downtime in the afternoon and I decided to check in on this patient. He explained to me his frustrations at the cardiologist and the healthcare team as they continuously focussed on his history of drug addiction during coordination of care. He stated, that side of him is "long gone" and now, he is an addictions 
counsellor hoping to help others who are going through what he did. He shared his journey of addiction, loss, recovery, taking control of his life, and finding meaning in being a counsellor. I asked, "Do the other staff know this?" He chuckled and said, "No, you're the first one. Everyone else just stops at the drug addict part of my life and stays there." Behind the words on the chart is a real human being with a plethora of experiences, and at times, healthcare providers much too often stop at one and attach a label on a patient. This was an eye-opening experience. I can tell from his experiences that he, too, embraced my philosophy of healing yourself is connected with healing others.

\section{My Experiences as a Care Recipient}

I was diagnosed with a chronic condition when I was a teenager. As a family caregiver, my mother took many days off work to take me to specialist appointments, to get my bloodwork taken, to go to the hospital to get diagnostic tests done, and much more. Although she never complained, I knew the stresses she faced while caring for me were difficult. Taking days off work and paying for treatment and medication frequently led to financial challenges. The time required to provide care often left little time for her to rest and care for herself. And the constant worries my mother had over my condition at times overwhelmed her emotionally. As healthcare providers focused on my health throughout the years, I realized that my mother's needs as a caregiver were rarely acknowledged or recognized. I knew too well that feeling of invisibility that my mother was also experiencing. Something that I will always remember was when I had pneumonia and was recovering at home. Pneumonia further complicated my health given my chronic condition, and I was essentially bed-bound for days due to fatigue, weakness, and pain. My mother took a sleeping bag and slept next to my bed on the ground during this time, so she was easily accessible to me whenever I needed assistance - despite her room being a few feet 
away where she could rest more peacefully in bed. Her commitment and love as a caregiver is something I will always cherish. I always say, she taught me everything I know about taking good care of someone!

\section{Unique Perspective}

I am grateful for these experiences as without them, I would not be who and where I am. I have navigated the Canadian healthcare system as both a patient and a caregiver, and experienced firsthand the hardships and beauty in receiving and providing best quality care and treatment. A pervasive theme in my caregiving experience was the notion of feeling invisible. This is not exclusive to my experiences, but instead, a common experience among family caregivers (Applebaum, 2015; Ansello \& Rosenthal, 2007; Carmeli, 2014). Family caregivers frequently feel hidden, isolated, and invisible due to minimal recognition in public policies (Carmeli, 2014; Gray, Robinson, \& Seddon, 2008). Thus, my unique perspective of the current healthcare system as a patient, a family caregiver, and a registered nurse inspired me to explore how to best utilize the many facets of nursing practice to improve service delivery and quality of care. Through this study, I hope to use research to inform and improve care for family caregivers, who are often the "invisible second patient" (Brodaty \& Donkin, 2009, p. 217). 


\section{CHAPTER ONE}

INTRODUCTION: USING HEALTH-RELATED MOBILE APPLICATIONS IN CAREGIVING FOR A FAMILY MEMBER WITH DEMENTIA 
"There are only four kinds of people in the world:

those who have been caregivers;

those who currently are caregivers;

those who will be caregivers;

and those who will need caregivers."

- Rosalynn Carter, 1997

(Former First Lady of United States, Champion for Caregiving, and Founder of the Rosalynn

Carter Institute for Caregiving)

This quote highlights how caregiving is intricately intertwined into our lives. This life experience of caregiving is particularly prominent for those who have a family member living with dementia due to the nature of the condition. In this chapter, I provide a two-part background on the study phenomenon. In Part I, I first, provide an overview of dementia; second, discuss family caregiving in the context of dementia; and last, describe how mobile technologies have influenced the family caregiving experience. In Part II, I situate the study by providing the problem statement, the research purpose and questions, as well as outlining the significance of the study.

\section{PART I: BACKGROUND}

Dementia is a syndrome caused by neurodegeneration and characterized by progressive deterioration in cognitive ability. This deterioration may manifest in symptoms such as difficulties with memory, managing behaviour, problem-solving, judgment and decision-making (Alzheimer's Disease International, 2010; Alzheimer Society of Canada, 2010; Prince et al., 2013). The inexorably progressive nature of the symptoms has a significant impact on an individual's independence, self-care abilities, daily functioning, and general quality of life, 
making dementia a leading contributor to impairment and dependence worldwide (Alzheimer Society of Canada, 2010; Alzheimer's Disease International, 2010; Prince et al., 2013). The most common type of dementia is Alzheimer's disease, while other underlying pathologies include dementia with Lewy bodies, vascular dementia, frontotemporal dementia, Creutzfeldt-Jakob disease, Parkinson's disease, and Huntington's disease (Alzheimer's Disease International, 2010; Alzheimer Society of Canada, 2017c).

On May 29, 2017, a global action plan on the public health response to dementia from 2017 to 2025 was endorsed by delegates at the World Health Assembly (World Health Organization [WHO], 2017). This global action plan's objective is to improve the lives of persons living with dementia, their families, and people who provide dementia care as well as to alleviate the impact associated with disease on communities and countries around the world (WHO, 2017). The action plan provides a comprehensive blueprint for action, covering areas of: lowering the risk of dementia; improving diagnosis, addressing treatment and care; promoting awareness of dementia; implementing dementia-friendly initiatives; advancing research and innovation; and increasing support for caregivers of persons living with dementia (WHO, 2017). WHO asserts that dementia is a public health priority and is committed to mobilizing communities and countries worldwide to develop strategies and implementation plans.

\section{The Impact of Dementia on Canadian Society}

In Canada, there are currently 564, 000 individuals living with dementia, and this number is expected to double in the next 15 years (Alzheimer Society of Canada, 2017b). According to the Alzheimer Society of Canada (2010; 2017b), dementia is the most significant cause of impairment among Canadians aged 65 and older with dementia care costs of $\$ 10.4$ billion each year. Alzheimer's Disease International (2016) reports that the costs for persons living with 
dementia in Canada are estimated to be over five times greater than older people living without dementia. To keep pace with the shifting demographics whereby 1.4 million Canadians are estimated to be diagnosed with dementia in the next few decades, dementia care costs are predicted to increase exponentially to $\$ 293$ billion per year by 2040 if current practices continue (Senate of Canada, 2016).

On June 22, 2017, the passing of Bill C-233, “an act respecting a national strategy for Alzheimer's disease and other dementias", by the Canadian government made Canada the $30^{\text {th }}$ country to launch a national dementia strategy to address the impact of dementia on Canadian society (Alzheimer Society of Canada, 2017d, para. 1). This means that the Canadian government is committed to "actions with definitive timelines, targets, reporting structures and measurable outcomes" (Alzheimer Society of Canada, 2017d, para. 2). Thus, it is expected that current practices will change to incorporate potential components of dementia strategies such as raising awareness, enhancing coordination of care, providing research funding, improving training for healthcare professionals, and sharing best practices (Alzheimer Society of Canada, 2017d). A national dementia strategy will ensure all Canadians living with dementia and their families have access to the same quality of care regardless of their location, thereby preparing Canada to meet the challenges associated with dementia with a "coordinated, focused approach to care and research" (Alzheimer Society of Canada, 2017d, para. 8). In fact, in March 2019, the Government of Canada announced that they will provide $\$ 50$ million over five years to fund Canada's first national dementia strategy which will be used towards targeted campaigns and activities focusing on risk reduction and prevention, development of treatment guidelines and best practices, and investigation of the impact of dementia on Canadian communities (Alzheimer Society of Canada, 2019). 


\section{Family Caregiving to a Relative Living with Dementia}

For the last decade, there have been concerted efforts by policymakers to encourage older adults to age at home, resulting in aging in place initiatives gaining momentum throughout Canada (Lilly, Robinson, Holtzman, \& Bottorff, 2012). Examples of these initiatives include Hospital-at-Home (Leff, 2009), Home First (Giosa, Stolee, Dupuis, Mock, \& Santi, 2014), and Aging at Home (Giosa et al., 2014). The First Ministers' 2003 Accord on Health Care Renewal shifted government funds to support short-term acute home care and end-of-life services through cutbacks to long-term care investments for individuals living with chronic health conditions, such as dementia (Lilly et al., 2012). The role of family caregivers has become an increasingly critical means of meeting the demands of the aging population in Canada in light of this changing healthcare climate, limited health system resources and a focus on moving care to the home (Giosa et al., 2014). There has been a steady decline of formal, paid care utilization, and a growth in relying on family caregiving (Giosa et al., 2014). In 1997, the number of Canadians providing care to family members with long-term health conditions was estimated to be 2.85 million people whereas most recently, this figure has increased to over 8 million in 2012 (Canadian Caregiver Coalition, 2014; Giosa et al., 2014; Sinha, 2013). The cost of care would strain the Canadian healthcare system if not for family members contributing more than $\$ 25$ billion in unpaid labour annually in caring for their older relatives (Hollander, Liu, \& Chappell, 2009).

A high proportion of persons living with dementia require some form of care, ranging from assistance with instrumental activities of daily living to full support with personal care and around the clock supervision (Alzheimer's Disease International, 2010). With persons living with dementia primarily aging in place and residing at home, family members often become the 
main source of support as unpaid, informal caregivers (Brodaty \& Donkin, 2009; Lilly et al., 2012). In Canada, adult children (58\%) and spouses (32\%) most commonly take on the role of an unpaid caregiver of persons living with dementia (Canadian Institute for Health Information, 2018). In fact, up to $90 \%$ of in-home care is provided by Canadian family caregivers for persons living with dementia (Tam-Tham et al., 2016). Family caregivers have been described to be crucial for maintaining persons living with dementia in the community (Brodaty, Green, \& Koschera, 2003). Family caregivers providing care for persons living with dementia have been reported to make up for 7\% of all Canadian caregivers (or approximately 910, 000 individuals), making dementia the fourth most common condition to require family caregiving in Canada (Turcotte, 2013). Currently, there are over 400 million hours in informal, unpaid dementia care provided by Canadians each year (Alzheimer Society of Canada, 2017a).

The long-term, progressive nature of dementia places substantial strain and burden not only on healthcare providers, the healthcare system, and society in general, but most importantly, family caregivers (Alzheimer Society of Canada, 2010). There is a growing recognition that the aging in place movement has led to inadvertent negative consequences for some family caregivers of persons living with dementia (Lilly et al., 2012). Research has also shown that the current Canadian homecare policy environment has not only failed to adequately address the health and wellness needs of family caregivers, but also placed them in more desperate circumstances where they are struggling to receive recognition and adequate services as well as additionally burdened by expectations to look after themselves (Lilly et al., 2012).

A fundamental re-orientation towards the family caregiver is crucial by viewing family caregivers as an essential health human resource in a system that is dependent on their contributions, modifying existing services, and providing new ways of supports to improve 
outcomes for persons living with dementia and their family caregivers (Prorok, Horgan, \& Seitz, 2013; Lilly et al., 2012; Ward-Griffin, 2012). Experts have put forth many recommendations to address the "dementia epidemic" in Canada - one of which is to provide support to family caregivers (Alzheimer Society of Canada, 2010, p. 8). As family caregivers of persons living with dementia have become an integral part of our Canadian healthcare system, attending to their needs has become a key responsibility, one that can improve outcomes for both the individual living with dementia and their family caregivers. One uncharted area of support is related to the use of mobile technologies.

\section{How Mobile Technologies Have Influenced Family Caregiving}

Healthcare technology has been developed in order to enhance and supplement care across the health spectrum, encompassing the body, home environment, community, and caregiving (Center for Technology and Aging, 2014; Gibson, Dickinson, Brittain, \& Robinson, 2015). There has been widespread adoption of technological approaches around the world due to its ability to enhance the quality of care, improve access to healthcare services, and encourage patient participation in the management of their own health (Milani et al., 2016; Siminerio, 2010).

In Canada, the federal government created a comprehensive plan referred to as Digital Canada 150 and committed \$305 million over five years from 2014 to 2019 to help Canadians take full advantage of the opportunities of the digital age by increasing and improving access to internet and cell phone services (Government of Canada, 2015a; 2015b). This plan was

formulated with the belief that digital and telecommunications technologies would better enable Canadians in living more productive and heathier lives (Government of Canada 2015b). In particular, technology in the form of electronic health (eHealth) - "the application of information 
and communications technologies in the health sector" - is viewed as an essential element of healthcare renewal by the Government of Canada (2010). The Canadian government has been making investments in promoting the eHealth agenda with the strong belief that adopting eHealth systems would result in better outcomes for Canadians by improving the accessibility, quality and efficiency of the healthcare system (Government of Canada, 2010). Globally, as advancements in technology continue to progress, more and more developments of powerful and portable computing devices are taking place - warranting major transformations in the field of eHealth (Bhavnani et al., 2016).

One such transformation is the introduction of mobile health (mHealth), defined as healthcare services provided through mobile devices, due to the changing technological landscape in Canada and worldwide. The WHO (2011) further describes mHealth as "medical and public health practice supported by mobile devices, such as mobile phones, patient monitor devices, personal digital assistants (PDAs), and other wireless devices...” (p. 6). The ubiquity and widespread use of mobile phones has enabled researchers and clinicians to use them as a platform for the development of tools and techniques to address geographic barriers, shortage of healthcare providers, high healthcare costs, and most importantly, inequities and disparities in health service delivery and access (Iribarren, Cato, Falzon, \& Stone, 2017). Current evidence indicates that mHealth can improve patient care by enabling greater communication between clinicians, and from clinicians to patients; improving patient knowledge and understanding about their condition; facilitating greater patient engagement and partnership in their care; reducing healthcare costs; increasing access to health services; and monitoring patients remotely for disease management or health maintenance (Gleason, 2015; Moore, 2012; Semple \& Armstrong, 2017; Steinhubl et al., 2013). mHealth widens the potential market of healthcare services to a 
broad spectrum of end-users, from those who are motivated and healthy to those who have chronic conditions (Garcia-Gomez et al., 2014). In particular, it has been proposed to be an efficient and economical method to address issues of caring for individuals with chronic conditions at home (Chiang \& Wang, 2016).

There are several categories of mHealth technologies available including smartphone health applications (mHealth apps) (Bhavnani et al., 2016). Currently, mobile apps are becoming an ideal and predominant platform in mHealth due to their popularity, increased sophistication, and connectivity (Iribarren et al., 2017). Because mobile apps can support added functionalities beyond the basic features of mobile phones (such as short message service [SMS], images, and videos), their potential for real-time data collection, interactivity, graphic feedback, and links to social functionalities make it the primary category of mHealth technologies used in healthcare delivery (Iribarren et al., 2017). It is important to note that a distinction between mobile apps and mHealth apps exist: mobile apps are software programs developed to run on mobile devices that provide specific services for the user (Merriam-Webster, n.d.), whereas mHealth apps is a category of mobile apps that focus on the provision of medical and public health services.

In Canada, the use of mobile devices in the form of smartphones and their apps have become common as their advanced hardware and software capabilities, comparable to those of desktop computers, enables the device to perform complex functions to meet the user's needs (BinDhim et al., 2014; Lieffers, Vance, \& Hanning, 2014). In 2016, for the first time, 75\% of Canadians own a smartphone, and over 30\% have at least one mobile app they use for health and wellness (Canada Health Infoway, 2017; Catalyst, 2016). In particular, one out of every two Canadian adults under 35 years of age have reported using a mobile app to manage their health or well-being in the previous three months (Paré et al., 2017). Users have expressed that using 
mobile apps helps them become proactive in their overall wellness and more equipped when meeting with healthcare providers (Canada Health Infoway, 2017).

Family caregivers of persons living with dementia are beginning to leverage available mobile apps to assist with caregiving designed by researchers, government organizations, healthcare institutions, charities and non-profit organizations in field of dementia. For instance, Alzheimer's Association (2013) provided a list of mobile apps on their website that can help both persons living with dementia and their family caregivers. Similarly, other organizations such as Family Caregiver Alliance (2012), DementiaToday (2016) and Young Dementia UK (n.d.) have also posted comprehensive lists of mobile apps that are useful for family caregivers to manage caregiving duties for their loved ones. In Toronto, the University Health Network (2017) also offers a list of free health and wellness mobile apps for patients and their families. Notably, funded by the Government of Canada's Social Development Partnership Program, Sinai Health's Reitman Centre for Alzheimer's Support and Training has developed a mobile app called "Dementia Advisor". This app is designed to help family caregivers learn how to manage difficult behaviours and improve communication and problem-solving skills through interactive, chat-based role playing (Toronto Central Healthline, 2017). Additionally, more mobile apps in dementia care were found in the literature targeted towards persons living with dementia and their caregivers (refer to Appendix A for more details). While there are increasingly more mobile apps designed for this specific population, little is known about the user experiences of family caregivers integrating mobile apps into their caregiving activities as no research to date has explored this phenomenon. 


\section{PART II: SITUATING THE STUDY}

\section{Problem Statement}

The number of family caregivers is expected to rise considering the increasing prevalence of dementia in Canada. In consideration of the need to support family caregivers, there is a growing effort to leverage mobile apps (a new form of technology) to improve the family caregiving experience in the dementia context. Despite high and increasing developments in mobile app technologies, end-users continue to experience barriers in technology adoption due to poor understanding of the user experience when designing and developing technology platforms

(Cimperman, Brencic, \& Trkman, 2016). There is a shift toward family-centered care as persons living with dementia often rely upon or desire the involvement of family caregivers in care (Kelly \& Wolff, 2015). Thus, it has become critical to incorporate family caregiver perspectives in care delivery as efforts to promulgate engagement in care must also address the needs of family caregivers (Kelly \& Wolff, 2015). Most importantly, family caregivers must be better incorporated in the development and evaluation of technological solutions to support persons living with dementia and their family caregivers (Kelly \& Wolff, 2015). Understanding the process of how and why family caregivers interact with and integrate mobile apps into their caregiving roles plays a pivotal role in successful mobile app implementation and thus, should be at the forefront of mobile app development (Cimperman et al., 2016). By exploring user experiences with current mobile apps, healthcare providers, researchers and app developers can better design future mobile apps to be more effective and accepted among family caregivers (Peng, Kanthawala, Yuan, \& Hussain, 2016).

Nevertheless, researchers have found that family caregivers (who are often the gatekeepers, or in other words, people who have the ability to allow access, for technology 
integration) are often missing during the technology design, development and evaluation process

(Gibson et al., 2015; Kinney et al., 2003; Shreve, Baier, Epstein-Lubow, \& Gardner, 2016;

Zapata et al., 2015). This is of particular concern given that a lack of a holistic and

comprehensive understanding of the user experience in designing technology has often led to a

disconnect between users' expectations and experiences in using such technologies and the

services they provide (Cimperman et al., 2016). Little research has been done to draw a complete

understanding of the family caregivers' user experiences of mobile apps. Specifically, there is no

research to date that explores the perceptions and user experiences of family caregivers of

persons living with dementia on using mobile apps to support their caregiving activities.

\section{Research Purpose and Questions}

The purpose of this study was to explore and describe the user experiences and perspectives of family caregivers of persons living with dementia on using mobile apps to assist with caregiving activities. For this study, the focus was on mobile apps, rather than only the category of mHealth apps. This focus was adopted because mobile apps are all-encompassing and the purpose of this study was to understand family caregivers' use of general mobile apps as a means to navigate the social world, rather than a limiting exploration of mHealth apps as a means to navigate medical and public health services. Additionally, research has shown that technology does not necessarily need to be purposely designed for dementia care as many mainstream technologies can be adapted by persons living with dementia and their family caregivers to address their changing needs (Meiland et al., 2017). In other words, some technologies developed not specifically for dementia can lend themselves well to support this population (Meiland et al., 2017). As such, it was decided to include the use of all mobile apps in this study in order to provide a more comprehensive understanding of how mobile apps can 
address various aspects and needs in the lives of persons living with dementia and their family caregivers.

The main research question is: What are the user experiences of family caregivers using mobile apps to assist in caregiving for their loved ones living with dementia in the community? Research sub-questions include: What are family caregivers' perspectives on the use of mobile apps to support caregiving activities? What are the influences that impact the use of mobile apps for family caregivers? How do family caregivers use mobile apps during their caregiving activities? What are family caregivers' experiences of using photographs as a conduit for explicating their user experiences?

\section{Significance to Policy, Practice, and Healthcare}

The findings of this study offer an understanding of the family caregiver's experiences of using mobile apps to support caregiving for a loved one living with dementia. This in turn can inform future mobile app developments tailored to address caregiver needs of family caregivers. Moreover, the findings are particularly valuable to technology developers, healthcare organizations, policymakers, and healthcare providers as they play a crucial role in developing, facilitating integration, and improving user experiences to maximize use of mobile apps.

Coupled with a comprehensive understanding of family caregivers' user experiences, mobile apps can be designed and employed to promote more effective caregiving and improved quality of life for care recipients and their family caregivers.

Support and information from health and social care professionals have been acknowledged as a key driver of technology use in dementia care (Gibson et al., 2015). However, family caregivers have expressed that they often receive little practical support as well as ongoing information and advice from health and social care professionals which has been 
identified as a major barrier to technology use in this particular group (Gibson et al., 2015; Newton et al., 2016). As a result, family caregivers are left to devise their own ways of integrating the technologies into caregiving practice (Gibson et al., 2015; Newton et al., 2016). Thus, by gaining a better understanding of the interplay of caregiving and mobile apps for family caregivers of persons living with dementia, health and social care professionals can provide informed support and information to facilitate full integration of mobile apps within the caregiver's routines. Successful mobile app implementation has the potential to not only lower healthcare costs (Iribarren et al., 2017), but most importantly, contribute to better health outcomes and quality of care for both the individual living with dementia and their family caregivers (Klimova, 2017). 


\section{CHAPTER TWO}

PHILOSOPHICAL UNDERPINNINGS 
"We do not see things as they are, we see things as we are"

- Talmudic Saying (Rabbi Aaron Parry, 2004, p. 176)

This Talmudic saying resonates strongly with my philosophical beliefs as I believe we, as human beings, see the world not as it is but as molded through the eyes of our prejudices, previous experiences, and preconceived notions. In other words, what we describe of the world around us is a reflection of ourselves, our perceptions, and our paradigms. As such, mirroring my philosophical influences, this study was underpinned by constructivism. In this chapter, I discuss the importance of philosophical underpinnings in research, provide an overview of the constructivism paradigm, and describe the philosophical assumptions of constructivism.

\section{PHILOSOPHICAL UNDERPINNINGS}

Philosophical underpinnings act as the foundation of how individuals think and study social phenomena. Subsequently, these underpinnings shape the methods and procedures for how researchers assemble and dissect data (Corbin \& Strauss, 2008; Creswell, 2013). In other words, these underpinnings provide the context and angle from which to approach a particular phenomenon, including the types of research questions to ask and how to gather data (Creswell, 2013; Crotty, 1998). The philosophical underpinnings of different methodologies employed in the pursuit of knowledge fall on a continuum - with extremes representing two distinct paradigms.

At one end lies the positivist paradigm, which embraces the use of a deductive process of measuring and analyzing phenomena as the way of knowledge acquisition (Streubert \& Carpenter, 2011). This paradigm is represented by quantitative methodologies which have historically been the dominant way of what counts as knowledge due to the heavy emphasis on the quantification of observations in science (Guba \& Lincoln, 1998). On the other end of the 
continuum lies the constructivist paradigm, which posits knowledge generation as a

fundamentally inductive and emerging process - the worldview in which qualitative methodologies are built upon (Streubert \& Carpenter, 2011). In qualitative research, the aim is to understand the human experience by exploring the elements surrounding events with self, other, and the environment. Notably, qualitative researchers recognize the influence of unique perceptions of individuals, the concept that people are active beings who construct their own realities, and the notion that actions, reactions, and interactions are dependent on the views of each individual (Munhall, 2012).

\section{SITUATED PHILOSOPHICAL FOUNDATION}

The Constructivism Paradigm

The philosophical foundation that underscores this qualitative study is naturalistic inquiry within the constructivism paradigm. As it is understood through the works of Guba and Lincoln (1998), constructivism considers all knowledge as reliant on human interactions within a social context and seeks to undertake research in the natural settings of participants (Appleton \& King, 2002). Meaning and knowledge in this worldview is constructed, not discovered (Guba \& Lincoln, 1998). More specifically, knowledge is constructed when an individual interacts with the world, and thus reality becomes a by-product of human perception. Meanings are negotiated socially and historically as they are formed through interaction with others, historical and cultural norms that operate in individuals' lives, and their environment (Creswell, 2013; Guba \& Lincoln, 1998). Subsequently, individuals' understanding of meaning is informed by environmental and social influences that impact their experiences, behaviours, and thinking (Crotty, 1998). Ultimately, this mode of inquiry explores the "labyrinth of human experience as 
people live and interact within their social worlds" and seeks to understand the diverse constructions that people possess (Appleton \& King, 2002, p. 642).

\section{Philosophical Assumptions}

Creswell (2013) specified four philosophical assumptions that shape and guide qualitative research, as well as form the basic belief systems known as paradigms. These assumptions are: ontology, epistemology, axiology, and methodology. Constructivism and its philosophical assumptions are what constitutes my philosophical perspective, and informed every step of the research process of this qualitative study.

The ontological assumption speaks to the nature of reality and its characteristics (Creswell, 2013). In qualitative research, the ontological stance is one that embraces the notion of multiple realities (Creswell, 2013). Similarly, in constructivism, the ontological assumption is relativism, which assumes that realities are socially and experientially based, as well as in the form of multiple and apprehendable mental constructions (Guba \& Lincoln, 1998, as cited in Creswell, 2013). This is consistent with my own ontological assumption which is centered on the multimodality of reality as I believe in the idea of multiple constructed realities. Participants present diverse perspectives and qualitative researchers aim to report such multiplicity. In this study, this can be seen in the use of multiple forms of evidence in themes showcasing words of different participants and presenting varying perspectives.

The epistemological assumption determines what counts as knowledge and how knowledge is known (Creswell, 2013). In qualitative research, knowledge is known through the subjective experiences of people and subjective evidence is assembled based on individual perceptions (Creswell, 2013). Qualitative researchers aim to become immersed in the data, lessen the distance between researcher and participants, and study participants in their natural setting. 
Through the close interaction between researcher and participants, the researcher becomes an "insider" (Creswell, 2013, p. 37) and an active player in the research process. In congruence, the epistemological assumption in constructivism is transactional subjectivism, which sees knowledge as created in interaction between researcher and participants (Guba \& Lincoln, 1998, as cited in Creswell, 2013). The end result is a partnership between the researcher and participants in creating knowledge as the researcher co-creates with the participants. In this study, I aimed to develop close interactions with participants as the participants and I are interlocked in an interactive process where we influence each other. The use of follow-up interviews that involved photo-elicitation interviewing also helped to lessen the distance between the participant and I as this method allowed me to gain a richer understanding of their experiences.

The axiological assumption pertains to the role of values in research (Creswell, 2013). As participants have values and are studied in their natural environments without the control or manipulation from researchers, qualitative researchers acknowledge the value-laden nature of their gathered data (Creswell, 2013). Qualitative researchers often "position themselves" in the study by discussing their values and biases, thereby recognizing their role as a research instrument since their values are intricately woven into the research data, analysis and findings (Creswell, 2013). I recognize that I cannot evade affecting the phenomenon under investigation and that I must acknowledge my positioning as only through subjective interpretation can an understanding of the phenomenon be uncovered. In other words, the study findings were socially constructed not only by the participants, but also by my role and contribution as a researcher. In this study, I acted as the primary data-gathering instrument (Lincoln \& Guba, 1985) and thus, it was imperative that my positionality as a researcher was described in depth in the prologue to 
make my existing beliefs and assumptions overt to myself and the readers (Creswell, 2013; Creswell \& Miller, 2000).

The methodological assumption is the process of research (Creswell, 2013). The approach to qualitative research is characterized as inductive, emerging and iterative (Creswell, 2013). This approach is also markedly shaped by the researcher's experiences in data collection and analysis (Creswell, 2013). The inductive nature allows for assembling data from the ground up as opposed to data being handed down entirely from a theory or the perspectives of the researcher (Creswell, 2013). Likewise, the methodological assumption in constructivism is hermeneutic-dialecticism, a process where individual constructions can be elicited and refined solely through interaction between researcher and participant (Guba \& Lincoln, 1998). The varying constructions are "interpreted using conventional hermeneutical techniques, and are compared and contrasted through dialectical interchange" (Guba \& Lincoln, 1998, p. 207, as cited in Creswell 2013). Thus, flexible emic-focused strategies (i.e. in-depth semi-structured interviews) were used to allow participants to share their constructions of the phenomenon under investigation due to the emergent nature of the study. In addition, an inductive and iterative approach to data analysis was used to promote interpretation of the data from the ground up.

Constructivism seeks to undertake research in natural settings and recognizes the complex interplay that helps to form, develop, and alter a person's constructions of any phenomena (Appleton \& King, 2002). Guided by constructivism, the purpose of this study was not to seek broad generalizable "truths" since many realities exist as individuals attribute their own interpretation and meaning to the phenomenon of interest. Instead, this mode of inquiry gives me the opportunity to understand the variety of constructions that individuals possess, aiming to achieve some consensus of meaning (Appleton \& King, 2002). Through this study, I 
strove to develop and present an understanding of the experiences of family caregivers by accessing the meanings participants ascribe to them. 
CHAPTER THREE

REVIEW AND SYNTHESIS OF RELEVANT LITERATURE 
"What good is the most sophisticated, well-developed technology intervention if the end-user does not use it?"

\section{- Diane F. Mahoney (2010, p. 66)}

Diane Mahoney, a well-known nursing researcher in the field of gerontology and technology (also known as gerontechnology), poses this question in her 2010 paper to emphasize the need to understand and include end-users in the design, development, and evaluation process of technology in order to meet its primary goal of actual use by end-users.

I want to preface this chapter by highlighting that my philosophical perspectives influenced the approach I took to reviewing the literature as I particularly sought out literature that explored experiences of family caregivers through qualitative methods. This is not to say that quantitative studies were not included in the review, but rather, it was important to understand the current state of knowledge of the chosen phenomenon in the qualitative paradigm. Given that majority of the research on mobile apps, technology, and user experiences are predominantly underpinned by the positivist paradigm, this literature review sheds light on the existing gap in the literature with respect to exploring the user experiences of family caregivers of persons living with dementia.

I focused my review and synthesis of the extant literature on three main areas in order to gain a comprehensive understanding of the interplay of mobile apps and family caregiving in the dementia context. Thus, the literature synthesis in this chapter is organized into three sections: (1) Experiences of family caregivers of persons living with dementia; (2) Technology and caregiving; and (3) Understanding the user experience in the dementia context. It was important to first review and synthesize the literature related to experiences of family caregivers unique to the dementia context in order to gain an understanding of how various forms of technologies, 
particularly mobile apps, can support and improve their caregiving experiences. Hence, in the first section, I reviewed recent research to identify salient themes that characterize the experience of family caregiving in the dementia context. In the second section, I reviewed the literature on technology in caregiving in general and then focused more closely on mobile apps in particular. Last, I reviewed and discussed research on the user experiences of family caregivers for technology and more specifically, mobile apps.

\section{Literature Search Strategy}

The search strategy and literature review framework as described by Kable, Pich and Maslin-Prothero (2012) was adapted for the purposes of this study and used to guide the literature search. The complete adapted version of the framework can be found in Appendix B. A critical review of the literature was conducted and focused on the main grounding questions: (1) What is the current state of knowledge on family caregiving for persons living with dementia?; (2) What is the existing knowledge on technology and caregiving?; and (3) What are the user experiences of family caregivers using technology and mobile apps?

In concordance with the framework, a systematic search was conducted which initially included literature from the following databases and search engines: Cumulative Index to Nursing and Allied Health Literature (CINAHL), Evidence Based Medicine Reviews, Medline, Nursing and Allied Health Database, PubMed, and Scholars Portal Journals. IEEE Xplore, an additional database in the field of information systems management was searched afterwards to gather more information on mHealth, mobile apps and user experience. Grey literature that met the inclusion criteria was included to provide a comprehensive body of knowledge.

The inclusion and exclusion criteria were informed by the PICO (Population, Intervention, Comparison, Outcome) and PICo (Population, Phenomena of Interest, Context) 
acronym as defined by the Joanna Briggs Institute (2011) for use in quantitative and qualitative studies, respectively. The inclusion criteria consisted of: English language, full text, studies published from 1997 (inclusive) to present, empirical studies from any geographical location, and articles from peer-reviewed journals. Of note, the literature review was conducted in 2017 and at the time, it was decided to search for articles within the last two decades to account for any seminal works on caregiving in the dementia context; thus, the inclusion criteria of year 1997 to present. Appendix C provides a more detailed outline of the inclusion and exclusion criteria employed for the literature review search. Keywords and subject headings used to search the scholarly databases include family caregiver, carer, informal caregiver, caregiving, dementia, Alzheimer's disease, mHealth, technology, mobile health, mHealth apps, smartphone, mobile applications, user experience, and user perceptions. The Boolean operators, OR and AND, were used to include alternate, synonymous terms and to join two key terms respectively. The search strategy involved screening titles and abstracts for relevancy for the intended study. The relevant articles were reviewed in their entirety along with their respective reference list to capture any additional relevant articles.

The Google search engine was also used to find grey literature by employing the listed keywords. The inclusion criteria comprised of: English language, full text, published from 1997 (inclusive) to present, and published by reputable government, healthcare, or non-profit organizations. Given that it is impossible to screen all retrieved results from Google searches, I relied on the power of relevancy ranking within Google search engines to bring the most relevant results to the top of the list (Godin, Stapleton, Kirkpatrick, Hanning, \& Leatherdale, 2015). I set the number of pages to be screened at five pages to ensure feasibility and consistency across 
searches. The first five pages of each search's hits (representing 50 results) were reviewed, by reading through the title and short text underneath.

The initial search from January 2017 to January 2018 generated 14,041 relevant articles in the scholarly databases and 200 relevant grey literature documents in the Google search. Using the same search strategy, another systematic search was conducted in June 2019 to update the literature review to include any literature that was published from January 2018 to June 2019. This second search generated 5511 relevant articles in the scholarly databases and 200 relevant grey literature documents in the Google search. After both searches, 147 final papers (with 5 from grey literature) were selected by evaluating the degree of relevance to the chosen phenomenon and the quality of the article by using the Critical Appraisal Skills Program (CASP; 2017) checklists. Refer to Appendix D for the PRISMA flow diagram summarizing my search strategy and Appendix E for a chart outlining all the references selected. Last, the reference list was checked for accuracy and web links accessed were updated.

\section{LITERATURE REVIEW AND SYNTHESIS}

\section{Background: Family Caregiving}

Formal caregivers, paid employees or volunteers connected to the social service and/or healthcare delivery systems, were the main acknowledged human health resource until the 1980s when the concept of family caregiver emerged to describe individuals who were caring for family members in their homes (Farran, 2002; Llanque, Savage, Rosenburg, \& Caserta, 2016). Research demonstrated that informal networks - predominantly family members-are the first resort as caregivers when a person encounters declining health (Chappell \& Funk, 2011). It is worth mentioning that informal caregiver, at times used interchangeably with family caregiver in the literature, is a more inclusive term referring to help provided by all non-professional 
providers of care, including family members, friends, and neighbours (Schumacher, Beck, \& Marren, 2006). In contrast, family caregiver refers to care provided within families. Other terms found in the literature that are associated with family caregiving include carers and family carers. For instance, Australia and the United Kingdom use the term "carer" to describe care delivered in a family setting (British Columbia Law Institute, n.d.). However, "family caregiving" remains the term most commonly used in Canada to capture unpaid care provided by family members at home (British Columbia Law Institute, n.d.). Additionally, as compared with the alternatives, the term "family caregiver" is used in this study because what the word "carer" represents has been described to be too broad as British Columbia Law Institute (n.d.) argues that "we may care for many people for whom we are not performing any caregiving activities" (p. 21). In other words, caregiving denotes an action which emphasizes on the actual act of providing care.

Two key reasons that placed family caregiving at the forefront of healthcare are the increasing numbers of individuals living longer and experiencing chronic conditions for a longer period of time as well as the changes in the healthcare delivery system (Farran, 2002). Furthermore, new healthcare reforms focusing on deinstitutionalization and cost-savings combined with new technologies has both significantly increased the number of individuals being cared for at home and also transformed the nature of the care provided (Armstrong \& Kits, 2001). As care recipients remain at home in increasingly more complex conditions, the home setting is transforming into a technologized caregiving environment whereby family caregivers may assume responsibilities typically ascribed to professional providers (Farran, 2002; Scott, 2001).

The types of caregiving and provision of care are described in various ways. Family caregivers provide a wide range of care which can be categorized into two broad areas: 
instrumental care and "invisible" work (Bowers, 1987; Schumacher et al., 2006; Scott, 2001). Instrumental caregiving is commonly recognized to include the traditional definition of caregiving, that is, the hands-on responsibilities (Bowers, 1987). These responsibilities are comprised of: (1) assisting with activities of daily living such as bathing, eating, and going to the washroom (Farran, 2002; Schumacher et al., 2006); (2) helping with activities requiring problem-solving skills, clinical judgment, and technological care such as carrying out treatment regiments and managing home dialysis (Schumacher et al., 2006, Scott, 2001); and (3) supporting of activities requiring organizational and communication skills such as navigating the healthcare and social services systems (Schumacher et al., 2006). Similarly, in terms of what constitutes as "provision of care" in the family caregiving context, Armstrong and Kits (2001) described that the care provided by family caregivers can be delineated into four broad, overlapping categories. These categories include: (1) care management (e.g. identifying and arranging formal care services, and managing finances); (2) assistance with instrumental activities of daily living (e.g. cooking, shopping, and household tasks); (3) assistance with activities of daily living (i.e. direct help with much more personal and medical aspects of care, such as dressing, bathing, eating, and medication administration); and (4) emotional and social support. The number of care provision categories that family caregivers undertake is dependent on life circumstances of the caregiver and the condition of the care recipient.

Bowers (1987) conducted seminal research into the concept of "invisible" work in caregiving to expand on the range of care provided by family caregivers. Bowers found that in addition to the traditional forms of caregiving, family caregivers also engage in "invisible" work which is less recognized and acknowledged. Bowers' study asserted the existence of four more conceptually distinct categories of family caregiving. First, anticipatory caregiving involves 
behaviours or decisions based on the anticipated, possible needs of the care recipient. Second, preventive caregiving includes activities for the purpose of preventing illness, injury, and complications through active monitoring and supervising. Third, supervisory caregiving includes active and direct involvement of the caregiver such as checking up on and arranging care for the care recipient. Lastly, described as one of the most difficult, yet important, types of care provided, protective caregiving's purpose is to protect the care recipient from threats to their self-image and self-perception as competent and independent. Caregivers also frequently attempt to protect the care recipient from becoming aware that they are being taken care of.

Lastly, I want to provide a brief background as to why family caregivers, as opposed to other forms of caregivers, were chosen as the focus of this study. In the dementia context, it is emphasized in the literature that much of the caregiving responsibility falls on family caregivers where the spouse being the most likely, followed by children and siblings (Etters, Goodall, \& Harrison, 2008; Yap, Seow, Henderson, \& Goh, 2005). Most persons living with dementia depend on their family caregivers to maintain in the community (Tam-Tham et al., 2016). Notably, family caregivers of persons living with dementia provide up to $90 \%$ of the care received in the home in Canada (Tam-Tham et al., 2016). As such, family caregivers play a unique and instrumental role in the overall management of dementia (Yap et al., 2005). They are an important source of information for the clinical assessment of the persons living with dementia as decisions made on how to best provide care for the them is often dependent on the information provided by the family caregiver (Yap et al., 2005). Importantly, the health outcomes and well-being of the persons living with dementia and the family caregiver are closely linked (Yap et al., 2005). Particularly, researchers found that kinship ties are associated with caregiver outcomes wherein closer kindship ties lead to increased caregiver burden (Etters et al., 
2008). Furthermore, the type of management strategies adopted by the family caregiver can impact the persons living with dementia's behaviour and well-being (Yap et al., 2005). For these reasons, researchers assert that there are always two clients in dementia care: the persons living with dementia and their family caregiver(s) (Yap et al., 2005).

\section{PART I: Experiences of Family Caregivers of Persons Living with Dementia}

The characteristics of a degenerative disease such as dementia often change the relational context between the caregiver and care recipient as caregivers experience a fundamental change in the loved one they are caring for, including changes in cognitive function and personality (Shim, Barroso \& Davis, 2012). As a result, family caregivers of persons living with dementia describe a wide spectrum of caregiving experiences which are organized in this section as the following themes that emerged from the literature review: (1) Challenging aspects of caregiving; (2) Positive aspects of caregiving; (3) Coping with family caregiving; (4) The influence of type of kinship on caregiving experiences; (5) Interdependency of the family caregiver and care recipient; and (6) Need for support.

\section{Challenging Aspects of Caregiving}

It was contended in the literature that while family caregivers of persons living with dementia possess some of the same characteristics as general family caregivers, there are greater effects on the former group in a variety of important domains (Ory et al., 1999). Clyburn and colleagues (2000) identified three dominant factors that contribute to challenging caregiving experiences unique to dementia: (1) care recipient factors (e.g. physical and cognitive decline, personality changes, and neuropsychiatric symptoms); (2) caregiver factors (e.g. caregiver's personality, cultural beliefs, perceived resources, and coping strategies); and (3) caregiving contextual factors (e.g. lack of social/community resources and family/support networks). 
Persons living with dementia generally require high levels of care associated with long care hours and physically demanding caregiving (Brodaty \& Donkin, 2009). Studies have found that $25 \%$ of family caregivers of persons living with dementia provide 40 hours of care or more per week as opposed to the $16 \%$ for other caregivers of non-dementia related conditions (Brodaty \& Donkin, 2009). Similarly, in Canada, the Canadian Institute for Health Information (2018) found that informal caregivers of persons living with dementia spend an average of 26 hours a week providing care compared to 17 hours for informal caregivers of older adults living without dementia. According to Ory and colleagues (1999), not only are caregivers of persons living with dementia more involved in caregiving with respect to hours of care per week, but also with the number of activities of daily living and instrumental activities of daily living tasks with which they provide support when compared to their other caregiver counterparts.

Given that caregivers of persons living with dementia provide more care and assist with a greater variety of activities than other caregivers, they are affected more negatively by their caregiving role when it comes to employment complications, mental and physical health problems, caregiver strain, family conflict, and decreased time for leisure (Connell, Janevic, \& Gallant, 2001; Ory et al., 1999). In terms of psychological well-being, Schoenmakers and colleagues (2010) found in their systematic review of 14 articles that the prevalence of depression in caregivers of persons living with dementia is significantly higher than in sociodemographically comparable groups of non-caregivers and in caregivers of individuals with nondementia related chronic illnesses or with a psychiatric diagnosis. Data from a more recent, cross-sectional study conducted by Karg and colleagues (2018) also indicated that informal caregivers of persons living with dementia experienced greater levels of caregiver burden, poorer mental health, and lower care-related quality of life when compared to informal caregivers of 
people with other chronic conditions. Similarly, del-Pino-Casado and colleagues (2019) found in their systematic review and meta-analysis of 55 studies that caregivers of persons living with dementia experience greater caregiver burden than those caring for frail older adults or stroke survivors. Confirming the trend in the literature, the Canadian Institute for Health Information (2018) reported that informal caregivers of persons living with dementia are more likely to experience distress than informal caregivers of older adults living without dementia.

Ory and colleagues aptly asserted that the unique experiences of caring for a loved one with dementia is attributed to the greater caregiver strain as they often have to contend with behavioural and psychological symptoms of dementia (BPSD) in the care recipient and the constant anticipation of issues arising in an unpredictable and uncontrollable manner. This is consistent with the findings from the Canadian Study of Health and Aging whereby the care recipient's behavioural disturbance is associated with increased levels of caregiver burden (Hebert, Dubois, Wolfson, Chambers, \& Cohen, 2001). Researchers have found a significantly higher prevalence of interrupted sleep in caregivers of persons living with dementia when compared to other caregivers (Capistrant, 2016). This is also supported by numerous studies as they reported that caregiving in the dementia context is more stressful than caring for an individual with a physical disability (e.g. Brodaty \& Donkin, 2009; Lilly et al., 2012) and caregivers of persons living with dementia were found to suffer increased levels of emotional distress associated with higher behavioural symptoms and cognitive impairment (Crespo, Lopez, \& Zarit, 2005; Mavounza, Ouellet \& Hudson, 2018).

Relative to other family caregivers, family caregivers of persons living with dementia are more vulnerable to social isolation, psychological and physical morbidity, and financial problems as a result of heavy demands of caregiving and challenging behaviours of care 
recipients (Brodaty \& Donkin, 2009; Lilly et al., 2012). Caregivers of persons living with dementia often experience feelings of social isolation due to the lack of social contact and support as they tend to sacrifice their leisure time and pursuits, restrict time with friends and family, and give up or reduce employment commitments (Brodaty \& Donkin, 2009). Furthermore, in the Canadian context, Alzheimer Society of Canada (2010) argued that the hidden unpaid costs borne by caregivers are substantial as indirect costs include hours spent caregiving as well as loss of earnings due to employment reduction or loss.

The research linking caregiving in the dementia context to negative health outcomes has been consistent as strain and burden can manifest as psychological and physical morbidity (Brodaty \& Donkin, 2009; Connell et al., 2001). When compared to other caregivers, caregivers of persons living with dementia experience significantly higher levels of psychological distress and stress as well as significantly lower levels of self-efficacy and subjective well-being (Brodaty \& Donkin, 2009). There are also increased risks for depressive symptoms (Connell et al., 2001), a higher prevalence of clinical depression and anxiety (Connell et al., 2001; Madaleno, Moriguit, Ferriolli, De Carlo, \& Lima, 2019), and increased psychotropic drug use among caregivers of persons living with dementia (Connell et al., 2001). With respect to physical morbidity, caregivers of persons living with dementia are at an increased risk of various health issues such as cardiovascular problems, lower immunity, slower wound healing, decreased engagement in preventative health behaviours, higher levels of chronic conditions, more doctor visits and use of prescription medications, and greater likelihood of poor sleep patterns (Brodaty \& Donkin, 2009; Van Mierlo, Meilan, Van der Roest, \& Droes, 2011). 


\section{Positive Aspects of Caregiving}

Much of the existing caregiving research documented the negative aspects of caregiving, but the positive aspects of caregiving have recently received increased attention in the literature (Hunt, 2003; Lloyd, Patterson \& Muers, 2016; Peacock et al., 2010; Schumacher et al., 2006). In fact, there is emerging evidence suggesting that the adaptation process of caregiving is characterized by the coexistence of both positive and negative experiences (Andrén \& Elmståhl, 2005; Yu, Cheng, \& Wang, 2018). Positive conceptualizations of the caregiving experience include uplifts of caregiving, caregiver satisfaction, caregiver esteem, finding or making meaning through caregiving, feeling a reciprocal bond, spiritual and personal growth, enjoying togetherness, and feelings of accomplishments and mastery (Brodaty \& Donkin, 2009; Hunt, 2003; Lloyd et al., 2016; Peacock et al., 2010). Notably, researchers suggested that seeking meaning from the caregiving situation which may bring feelings of helplessness, anxiety, or isolation is a choice that a caregiver can make (Shim et al., 2012). An increasing number of studies indicated that finding meaning in caregiving, also known as enrichment, is an effective method that leads to more positive experiences (Shim et al., 2012). Researchers have also found that some caregivers find gratification through perceiving their loved one living with dementia as reciprocating simply by the virtue of their continued existence, which often leads to better health outcomes for the caregiver (Shim et al., 2012). In the literature review conducted by Yu and colleagues (2018), four key domains of positive aspects of caregiving were found: (1) a sense of personal accomplishment and gratification; (2) feelings of mutuality in a dyadic relationships, (3) an increase in family cohesiveness and functionality; and (4) a sense of personal growth and purpose in life. Based on what is presented in the literature, perhaps the experiences of family 
caregiving, whether it is positive or negative, should not be considered at bipolar ends on a continuum, but rather regarded as a case of coexistence (Yu et al., 2018).

\section{Coping with Family Caregiving}

To stay productively engaged in caregiving for a prolonged period of time, family caregivers often rely on a complex and dynamic set of cognitive, affective and behavioural coping responses to maintain psychological fortitude, regulate their emotions, and solve or improve the practical problems they face (Gottlieb \& Wolfe, 2002). In a review of the qualitative literature, Lloyd and colleagues (2016) found that experiencing caregiving as positive was influenced by factors such as acceptance of or coming to terms with the situation, adapting a positive caregiving attitude, maintaining commitment to their relative, and drawing strength from various sources and supports. Other studies have shown that caregivers often adopt emotionfocused coping strategies, such as emotional support, acceptance, humor, positive reframing, and religious support, which can lead to improved caregiver outcomes (Cooper, Katona, Orrell, \& Livingston, 2008; Gallagher et al., 2011). In contrast, other researchers have found that problemfocused coping strategies, another form of coping wherein the caregiver acts to change their relationship to the stressor, was associated with caregiver well-being, positive outcomes, and lower psychological distress (Di Matteri et al., 2008; Kneebone \& Martin, 2003). Caregiver coping resources identified in the literature include the individual (e.g. finding meaning, accepting reality), family and friends, and professionals or formal service agencies (Sun, 2014).

\section{The Influence of Type of Kinship on Caregiving Experiences}

Considerable literature addresses how caregiving experiences in the dementia context can differ based on the type of kinship between the caregiver and care recipient. Majority of research focused on the differences between spousal and adult child caregivers. Pinquart and Sorensen 
(2011) suggested that the differences in caregiving experiences can be attributed to the fact that spouses are more likely to be older, married, living with the care recipient, have less competing responsibilities, and possess a different emotional relationship with the care recipient when compared to adult child caregivers. Similarly, Conde-Sala and colleagues (2010a) contended that spouses often regard caregiving as parcel of the marital commitment and thus, the caregiving role is assumed more naturally without resulting in strong feelings of burden. Other researchers also noted that spouses view caregiving as normative and part of their marriage vows (i.e. "in sickness and in health") whereas adult child caregivers experience the burden of role reversal and view caregiving as a significant change to their lives (Chappell, Dujela, \& Smith, 2014; CondeSala, Conde-Sala, Garre-Olmo, Turro-Garriga, Vilalta-Franch, \& Lopez-Pousa, 2010b; Lee \& Smith, 2012).

Although the caregiver relationship to the care recipient is often reported as amongst the potential factors impacting the caregiving experience, findings of studies have been inconsistent and conflicting. In some studies, researchers found that adult child caregivers experienced higher levels of burden due to having multiple demands and competing responsibilities, such as maintaining a job and caregiving for children of their own (Ashwill, Mulhall, \& Johnson, 2015; Chappell, Dujela, \& Smith, 2014; Conde-Sala, Garre-Olmo, Turro-Garriga, Vilalta-Franch, \& Lopez-Pousa, 2010a; Conde-Sala et al., 2010b; Reed et al., 2014; Springate \& Tremont, 2012). The authors noted that spousal caregivers, in contrast, adapt with the incremental changes in disease severity and gradually adjust to this way of living. Conde-Sala et al. (2010b) found that adult child caregivers would experience notable generational differences with regards to the care recipient and may feel more emotionally distant whereas spousal caregivers would be closer, both physically and emotionally, to the care recipient. As a result, their study results showed that 
spousal caregivers had a more positive perception of their quality of life than adult child caregivers.

In contrast, other researchers reported that spouses experience more burden than adult child caregivers (Andrén \& Elmståhl, 2008; Hong \& Kim, 2008; Kim, Chang, Rose, \& Kim, 2012; Ott, Sanders, \& Kelber, 2007; Rinaldi et al., 2005). According to Ott and colleagues, spousal caregivers experienced more sadness and longing, worry and isolation, and personal sacrifice burden than adult child caregivers. Kim and colleagues (2012) explained that higher levels of burden were not unexpected as the spouse tends to live with the care recipient, provide more hours of caregiving, and experience greater physical and emotional closeness to the care recipient. Other researchers suggested that spousal caregivers may have greater perceived burden of care due to their own declining health and aging as they often suffer age-associated chronic illnesses which makes providing care more difficult than an adult child (Hong \& Kim, 2008; Rinaldi et al., 2005). Despite the conflicting findings amongst studies, what is consistent throughout the literature is that caregiving experiences can differ based on the nature of the kinship. As such, the caregiver's relationship to the care recipient should be considered when developing and implementing interventions to improve health outcomes.

\section{Interdependency of the Family Caregiver and Care Recipient}

Family caregiver well-being is a crucial facet of dementia care given that the care of the individual living with dementia is often dependent on the family member who provides the care. Researchers have emphasized that the well-being of the family caregiver can directly impact the health outcomes of the care recipient (Lilly et al., 2012; Lwi et al., 2017; Papastavrou et al., 2007; Quinn et al., 2019). Family caregivers who report higher levels of positive aspects of caregiving experience lower levels of depression and anxiety as well as decreased impact of the 
behaviours of the persons living with dementia on their emotions (Lloyd et al., 2016).

Additionally, those who expressed higher satisfaction with caregiving were also less likely to institutionalize their loved one living with dementia (Lloyd et al., 2016).

On the contrary, due to the extensive care that caregivers of persons living with dementia provide, decreased caregiver health and quality of life often translate to poor outcomes for the family member living with dementia and earlier admission to institutional care (Lilly et al., 2012; Papastavrou et al., 2007; Vandepitte et al., 2018). Research has shown that high caregiver stress was associated with lower quality of life reported by persons living with dementia (Quinn et al., 2019). Lwi and colleagues (2017) also found that caregivers of persons living with dementia oftentimes experience declines in mental health, a factor that predicted greater care recipient mortality even when accounting for key risk factors in care recipients (e.g. diagnosis, age, sex, condition severity). In a systematic review of 81 studies conducted by Stall and colleagues (2019), the findings indicated that informal caregiver distress is commonly associated with important care recipient health outcomes, including elder abuse, worsening behavioural and psychological symptoms, deterioration of quality of life and function, and institutionalization. In keeping with the literature, data from the Canadian Study of Health and Aging showed that severe caregiver burden is significantly associated with institutionalization of the care recipient (Hebert et al., 2001), a finding that is supported by Alzheimer Society of Canada (2010). As such, maintaining the caregivers' health will be crucial to support the needs of this growing population. Efforts to identify and reduce poor caregiver outcomes have become an important healthcare issue that needs to be addressed. 


\section{Need for Support}

It is suggested in the literature that providing caregivers with adequate supports to meet their caregiving needs can expand coping skills, decrease caregiver burden and loneliness, improve attitudes towards the care recipient as well as enhance caregiving competence and confidence - which ultimately can foster positive caregiving experiences and improve caregiver outcomes (Stewart et al., 2006; Van Mierlo et al., 2011). According to Novais and colleagues (2017), through their systematic review of quantitative and qualitative studies, they found that family caregivers' need for support can be categorized into five main areas including: (1) information support on the disease, on pharmacological and non-pharmacological treatment, and on available community-based supports; (2) psychological support, such as psychological and emotional support for the care recipient and the family caregiver; (3) social support, including institutionalization, financial and legal issues, respite and day care, community services related to home support; (4) psycho-educational support, such as coping with behavioural and psychological symptoms of dementia, communication with the care recipient, stimulating and appropriate activities, and caregiver training; and (5) other support needs, including environmental safety, incontinence, medication management, and sexuality and intimacy.

Researchers have indicated that programs and interventions tailored to the caregiver needs and focused on improving the caregiving experiences have resulted in: lower rates of institutionalization for care recipients (Eloniemi-Sulkava et al., 2001); reduced frequency of and reactions to behavioural and psychological symptoms of dementia (BPSD) (Chua \& Pachana, 2016; Hebert et al., 2003); improved caregiving competence (Chiu, Wessen, \& Sadavoy, 2013; Devor \& Renvall, 2008); enhanced stress coping ability (Chiu et al., 2013; Milne, Guess, \& Russ, 2014); decreased caregiver burden (Devor \& Renvall, 2008; Hsu et al., 2017; Tremont, 
Davis, Bishop, \& Fortinsky, 2008); increased disease understanding (de Rotrou et al., 2011); lower psychological morbidity (Hsu et al., 2017); increased awareness of available support services (Milne et al., 2013); and improved mental well-being (Chiu et al., 2013). In addition, researchers have found that such interventions can delay institutionalization for up to 1.5 years, noteworthy when considering the healthcare costs associated with it (Boots, de Vugt, van Knippenberg, Kempen, \& Verhey, 2014).

However, Forbes and colleagues (2008) found that delayed, under-utilization or inappropriate utilization of community-based supports is pervasive theme with caregivers of persons living with dementia in Canada due to an array of barriers. Other researchers echoed the same findings where caregivers reported the following as reasons for non-use: inconvenient hours of operation (Brodaty, Thomson, Thompson, \& Fine, 2005), inaccessibility (Ward-Griffin et al., 2012), financial constraints (Brodaty et al., 2005; Lai \& Surood, 2008; Morgan, Semchuk, Stewart, \& D'arcy, 2002; Ward-Griffin et al., 2012), transportation challenges or costs (Colantonio, Kositsky, Cohen, \& Vernich, 2001), lack of respite (Colantonio et al., 2001), time constraints (Colantonio et al., 2001), and geographic location (Brodaty et al., 2005; Morgan et al., 2002). Further validating existing research, Lewis and colleagues (2010) highlighted that caring for a loved one living with dementia constitutes as another barrier as caregivers find it challenging to leave their family member to attend in-person sessions.

Because of the various barriers mentioned above, researchers, health and social care professionals, and healthcare institutions have started to consider other means of providing supports - such as novel forms of technology - for family caregivers in addition to the traditional care provision methods (Boots et al., 2014; Colantonio, Cohen, \& Pon, 2001; Forbes et al., 2008; Washington, Meadows, Elliot, \& Koopman, 2011). Research suggested that family caregivers 
are open to receiving caregiving support through technological platforms (Peterson, Hahn, Lee, Madison, \& Atri, 2016). More specifically, technology-based interventions have shown to increase access for participation as family caregivers can access supports in the privacy of their own homes without leaving their loved ones alone (Boots et al., 2014). Technology can also reach those who have challenges accessing traditional services (Boots et al., 2014). The use of technology in caregiving is discussed in further detail in the next section.

\section{PART II: Technology and Caregiving}

The growth of research and development in the area of innovative uses of technology in healthcare has illustrated its potential to offer many possibilities to improve health outcomes and quality of life for persons living with dementia and their families (Buettner, Yu, \& Burgener, 2010; Lorenz, Freddolino, Comas-Herrera, Knapp \& Damant, 2019; Smith \& Mountain, 2012; Topo, 2009). For instance, Smith and Mountain discussed the prominence of technologies in a number of life domains for persons living with dementia and their families, including technologies to (1) assist in diagnosis; (2) improve memory function and cognitive simulation and rehabilitation; (3) enhance aspects of safety and security; (4) facilitate access to information and support; and (5) enable activities for daily living. Technology has been identified as a convenient, accessible, and low-cost alternative for delivering interventions, providing support, and meeting the caregiving needs of family caregivers of persons living with dementia (Godwin, Mills, Anderson \& Kunik, 2013). These characteristics of technology-based interventions are particularly valued by family caregivers and significantly influence their decision to use the technology (Mao, Chang, Yao, Chen, \& Huang, 2015).

To evaluate the impact of various forms of technology on caregiving for persons living with dementia, several researchers conducted reviews to assess the benefits of a range of 
technologies on caregiver and care recipient outcomes. After conducting a literature review of technology for caregiving (e.g. telephone- and internet-based interventions), Topo (2009) reported positive findings of increased coping skills and social support as well as decreased caregiver burden and stress. The findings are consistent with what Powell and colleagues (2008) found in a systematic review a year earlier. Powell et al. also highlighted that technology-based interventions have effects on improving caregiver stress and depression, thereby showing potential to alleviate burden of caring and prolong community living.

In a systematic literature review of technology-based interventions used in individuals with Alzheimer's disease, Buettner and colleagues (2010) reviewed ten studies using various forms of technology, including computer touch screens, a combination of a digital clock and memory book, in-home technology support systems, computer-based multimedia programs, motion sensors, interactive video monitoring systems, and computer games. The review outcomes included positive effects for functional behaviours, recall of routes in the environment, recall of appointments, dates, or tasks, cognitive performance, social interaction, and medication adherence. In addition, positive outcomes for the caregivers were also noted, such as increased compassion for the care recipient as well as more free time and peace of mind. Buettner and colleagues asserted that technology-based interventions are an exemplary method to assist family caregivers, reduce healthcare costs, and minimize the need for professional support as well as stimulate activity and functions specifically impacted by dementia, thereby improving neuronal preservation and regeneration.

Similarly, a systematic review of technology-driven interventions for caregivers of persons living with dementia conducted by Godwin and colleagues (2013) reported that caregivers responded positively to technology-based programs to address their needs. The studies 
reviewed showed promising results relating to reductions in burden, improvement in overall mental health, as well as increased competence and confidence in caregiving skills (Godwin et al., 2013). Supporting the trend in previous studies, more recent reviews have also found that technology-based interventions can lead to positive outcomes for the care recipient, including reducing BPSD (Klimova, Valis \& Kuca, 2018), maintaining quality of life (Klimova et al., 2018), promoting social inclusion and well-being (Maresova et al., 2018), prolonging their autonomy (Maresova et al., 2018), as well as for the family caregiver, such as mitigating mental and physical burden (Hopwood et al., 2018; Klimova et al., 2018), and reducing anxiety and depression (Hopwood et al., 2018; Zhao et al., 2019). Further substantiating these findings, additional studies have also emphasized that caregivers are receptive to using technology-based tools to assist with caregiving roles and recognize the potential of caregiving technologies to improve their own quality of life, increase their feelings of being effective as a caregiver, and reducing the burdens and stresses of caring for loved ones (e.g. Benefield \& Beck, 2007; Lapid et al., 2015; Lee, 2015; Shreve, Baier, Epstein-Lubow, \& Gardner, 2016; Torp, Hanson, Hauge, Ulstein, \& Magnusson, 2008).

\section{Mobile Apps, Persons Living with Dementia, and Caregiving}

There is a common assumption that persons living with dementia are not capable, or will encounter much difficulty, learning and using new technologies due to its complexity and the cognitive capabilities needed with technology adoption (Kerkhof et al., 2016; Smith \& Mountain, 2012). However, studies conducted in the last decade showed that persons living with dementia are capable of learning to use new and everyday technologies, such as digital organizers and smartphones (Kerkhof et al., 2016; Nygard, 2008). Nygard and Starkhammer (2007) argued that persons living with dementia at times lack adequate knowledge and 
understanding, as opposed to being fearful or resistant of, new and advanced technologies and have demonstrated a positive attitude towards receiving instructions and learning. Similarly, a phenomenological study conducted by Nygard (2008) exploring how persons living with dementia experience the meaning of their everyday technology showed that technology was highly significant for persons living with dementia. Technology brought about practical and existential meaning for persons living with dementia, as it could assist them in various aspects of daily life (e.g. enabler of social interaction) as well as support how they perceive and present themselves (e.g. technology use as a symbol of normality in contemporary society).

Smartphones, tablets, and mobile devices that possess touch screens, provide a good interface for mHealth purposes as this form of technology is often intuitive, user-friendly, and "forgiving of mistakes" (Kerkhof et al., 2016; Smith \& Mountain, 2012, p. 338). Researchers have found that these characteristics make mobile devices effective tools for older adults, especially individuals living with dementia (Kerkhof et al., 2016; Smith \& Mountain, 2012). Several studies have shown that persons living with dementia were able to learn to use smartphones and tablets as well as its apps; found it user-friendly and valuable; and viewed it as a positive impact on their everyday living, self-management abilities, self-confidence, and involvement in society (Bier, Paquette, \& Macoir, 2018; Imbeault, Langlois, Bocti, Gagnon, \& Bier, 2018; Kerkhof et al., 2016; Kong, 2015). Researchers and clinicians have developed apps for persons living with dementia which have shown to be effective in supporting the five areas of self-management conceptualized by Martin and colleagues (2013): (1) relationship with family, friends and caregivers; (2) active lifestyle; (3) psychological well-being; (4) techniques to cope with memory losses; and (5) information about dementia. The ability for persons living with dementia to use mobile devices independently is important as this can provide meaningful 
activities for them to do while caregivers do other things, which can potentially reduce caregiver burden (Tyack \& Camic, 2017).

Studies have found that family caregivers of persons living with dementia were overwhelmingly receptive towards smartphone-based interventions aimed at reducing the psychological burden and social isolation inherent to caregiving, increasing access to resources and supports, and facilitating their ability to ensure the care recipient's safety and monitor the progression of their condition (Lee et al., 2017; Shreve et al, 2016). According to Lee and colleagues (2017), family caregivers of persons living with dementia, particularly in ethnic minority groups, use smartphones and apps more often than computers. The authors asserted that smartphone-based interventions could address the needs of family caregivers who cannot attend in-person interventions due to caregiving and/or transportation issues. Similarly, other researchers have emphasized that mobile apps can alleviate geographic (e.g. in-person support groups), physical (e.g. transportation and respite care needs), time (e.g. flexible scheduling and no time needed for travel), or financial (e.g. travel expenses) barriers (Silva, Rodrigues, de la Torre Díez, López-Coronado, \& Saleem, 2015), enabling family caregivers to meet their needs in order to enhance their caregiving experiences. In fact, 25 studies were found that discussed the development process of specific mobile apps by researchers to assist persons living with dementia and their caregivers in domains such as care provision, management, quality of life, prevention, and awareness. Refer to Appendix A for more information on all the studies, including a description of the app, the development methods, and significant findings. The studies reported positive results overall and emphasized the potential for mobile apps in dementia care. Specifically, results from the studies suggested that persons living with dementia 
and their caregivers were receptive to the designed mobile apps as well as found them valuable and useful in care provision.

\section{PART III: Understanding the Mobile App User Experience in the Dementia Context}

The definition for user experience put forth by the International Organization for Standardization (ISO; 2010) 9241-110:2010 (clause 2.15) is the most standardized in the literature: "a person's perceptions and responses that result from the use and/or anticipated use of a product, system or service". This formal definition is supplemented by other interpretations. Park and colleagues (2013) contended that user experience is broadly defined as "all aspects of the interaction between a user and a product" (p. 187). Khan (2012) asserted that user experience focuses on a more subjective and holistic view on that interaction. Similarly, Law and colleagues (2009) characterized user experience as dynamic, context-dependent, and subjective. These various interpretations of user experience provided a comprehensive understanding of the concept and were used to inform the study.

It is important to understand the end-users and their user experiences in the complete development process (Davis \& Oakley-Girvan, 2017; Nygard \& Starkhammer, 2007; Smith \& Mountain, 2012; Tamim \& Grant, 2016) as limited knowledge of the end-user can hinder user engagement and intervention effectiveness (Anderson, Burford, \& Emmerton, 2016; Laidlaw et al., 2017; Tamim \& Grant, 2016) and thus, result in technology abandonment rates as high as $75 \%$ to $80 \%$ among end-users (Liu et al., 2015). Described as gaining an understanding of how people interact with products and other people, user experience is an exceedingly critical issue as it can assist health and social care professionals and technology developers to create products that will improve the lives of those who use the technologies (Forlizzi \& Battarbee, 2004). 
After conducting reviews, many researchers have found that only a few studies have used a user-centered design in the development of mobile apps (e.g. Bateman et al., 2017;

Rathnayake, Moyle, Jones, \& Calleja, 2018). In particular, in a systematic review, Bateman and colleagues (2017) found that a large number of studies did not incorporate the end-users' input during the development of mHealth interventions for persons living with cognitive impairment (i.e. dementia). They asserted that this can create potential mismatch between the proposed solution and the end-users' needs as well as highlighted the need to have a strong understanding of the end-users' characteristics, goals, tasks, needs, capabilities and contexts. Thus, the significance of understanding the user experience is especially emphasized in caregiving in the dementia context, as it is imperative to match the technology capabilities to actual needs of caregivers, understand the preferences of how caregivers want to interact with the technology, and learn how it can integrate into caregivers and their care recipient's lives without presenting additional burdens related to technology use (Benefield \& Beck, 2007). After having extensive experience in spearheading research projects aimed to design and develop remote monitoring technologies for older adults and their caregivers, Mahoney (2010) further emphasized the need for technologies to be tailored to the concerns of end-users in order to promote adoption and buyin.

However, only a few studies have explored caregiver needs and preferences with respect to technology in the field of dementia caregiving. In a 2001 study, Colantonio and colleagues, through telephone interviews, explored the use and interest in support strategies such as telephone, newsletters, and computer services, among caregivers of persons living with dementia. The findings showed substantial interest in the use of the three strategies by caregivers. While this study provided valuable insight into the end-user needs of caregivers, this 
study was conducted more than 18 years ago, limiting its ability to reflect the latest trends in caregiver perceptions and technology development.

Years later, a study investigating the perceptions of family caregivers of persons living with dementia regarding a range of technologies through self-administered questionnaires was conducted by Rialle and colleagues in 2008. 270 family caregivers completed the questionnaires, which reflected that technologies most desired were ones that increased the care recipient's safety and caregiver's social connectedness. However, the setting (greater Paris, France area) and the time of the study limits its applicability to the current Canadian context despite its provision of a pioneering insight into the technology needs and preferences of family caregivers of persons living with dementia.

In Canada, Czarnuch and Mihailidis (2011) employed an 85-item questionnaire to explore the needs and the roles of intelligent assistive technology to support family caregivers of persons living with dementia during activities of daily living. It is important to note that assistive technology is the umbrella used to describe "products, equipment, and systems that enhance learning, working, and daily living for persons with disabilities” (Assistive Technology Industry Association, n.d., para. 1) while intelligent assistive technologies are assistive technologies that emerged due to recent advancements in artificial intelligence and have its "own computation capability and the ability to communicate information through a network" (Ienca et al., 2017, p. 1302). Respondents in the study indicated that they preferred intelligent assistive technologies to be autonomous, familiar, simple and unobtrusive; thus, shedding light on family caregiver needs of technology from a Canadian perspective.

In a qualitative study conducted by Rosenberg et al. (2012), spousal caregivers reported an overall positive attitude and readiness toward using technology in their caregiving roles. The 
findings provided more information on the spousal caregivers' conceptions of technology (e.g. utility perspective on technology) and the conditions for incorporation of technology (e.g. experiencing a need, integration in habits, simplicity, and non-stigmatizing). Similarly, Gibson and colleagues (2015) conducted a qualitative study using semi-structured interviews to explore the everyday use of assistive technology by persons living with dementia and their families. Topic areas explored in the interviews include general feelings about the use of technology to assist persons living with dementia as well as current use or non-use of everyday technology. The findings indicated that assistive technologies play an important role in supporting families to continue caring. Persons living with dementia also reported positive feelings towards assistive technology, especially if it can facilitate autonomy and independence. In the same year, Mao et al. (2015) investigated caregivers' perceived usefulness of available assistive technology devices by administrating a questionnaire that allowed them to rate 82 devices (e.g. voice reminders, camera monitoring system) as low or high-perceived usefulness to caregivers. Mao and colleagues found that caregivers were generally receptive to the usefulness of the listed devices and describe accessibility and cost-effectiveness as key factors for influencing their decision to use the device.

More recently, in a qualitative study that explored the experiences of using assistive technology in everyday life among family caregivers of persons living with young-onset dementia, Holthe and colleagues (2018) found various benefits of the technology for the caregiver, especially when the technology is simple and easy-to-use, but also some barriers for successful use. A main finding was that a committed caregiver was integral in giving continual support and ensuring that the technology functioned as intended for the care recipient. The findings also underscored the importance of professional advice and support as well as an 
understanding of the needs, capabilities and preferences of the end-users. In another qualitative study, Ruggiano and colleagues (2019) employed in-depth interviews to explore how family caregivers of persons living with dementia use technologies and how technological functions can best meet their needs in navigating service systems they interact with to engage in caregiving (e.g. family members and healthcare providers). Participants commented on a variety of ways they found technology to be helpful for caregiving and provided suggestions for new methods that technology could be designed to enhance the quality of interactions within the system of stakeholders who are involved with their relative living with dementia's care. The findings highlighted that technologies developed for caregivers need to be effective, easy to use and more widely disseminated.

\section{Conclusion}

Different user groups have diverse user needs, preferences, and experiences; thus, user requirements vary significantly (Topo, 2009). Additionally, the context in which a mobile app is used is critical to its perceived usefulness and user experience. Specifically, contextual factors include the particular activity for which the app is designed, the characteristics of the targeted users (e.g. family caregivers), when the app is mostly likely to be used (e.g. routinely), and where the app will be used (Mao et al., 2015). As such, in line with the constructivism paradigm underpinning this study whereby knowledge is discovered through human interactions within a social context, a portion of the literature review focused on the various aspects of the family caregiving experience unique to the dementia context to gain a better understanding on how mobile apps can support persons living with dementia and their family caregivers. To increase the acceptability and actual use of mobile apps by family caregivers, it is important to understand 
not only their user experiences, but also the specific contexts in which they are used in their caregiving roles.

However, while the studies reviewed provided important insight into the user needs, preferences, and experiences of technology-based interventions from family caregivers of persons living with dementia, no studies to date have explored the user experiences of family caregivers of persons living with dementia on using mobile apps in their caregiving role. Thus, guided by constructivism, the purpose of this study to explore the user experiences of family caregivers is rooted in the fact that user experience research takes a holistic view of users' interaction with technology in social contexts of use, emphasizes the situational and dynamic aspects of using technology, as well as focuses on the dynamics of experience and how technology, characteristics of the individual, and context work together in shaping the experience of use (Bargas-Avila \& Hornbæk, 2011). As a result, the research approach assumed in this study uniquely adds to the current state of knowledge about the interplay of family caregiving and mobile apps in the dementia context, which can inform future mobile app designs and developments by researchers, health and social care professionals, healthcare organizations, and app developers. 


\section{CHAPTER FOUR}

RESEARCH METHODOLOGY, STUDY METHODS AND RESEARCH PROCESS 
"I want to understand the world from your point of view.

I want to know what you know in the way you know it.

I want to understand the meaning of your experience, to walk in your shoes, to feel things as you

feel them, to explain things as you explain them.

Will you become my teacher and help me understand?"

- James P. Spradley (1979, p. 34)

A major strength of the qualitative approach lies in its ability to explore one's

interpretations of the world and their experiences, affording readers to grasp the idiosyncrasies of their experiences. The way Spradley positioned himself as a learner with the participant as the teacher reflects the stance I took while conducting this study. To understand the qualitative approach I employed in the study, I have organized this chapter into three sections: (1) Part I: Research Methodology; (2) Part II: Study Methods; and (3) Part III: Reflexivity and Trustworthiness.

\section{PART I: RESEARCH METHODOLOGY \\ Qualitative Description}

In this study, I employed the qualitative description approach as described by Sandelowski $(2000,2010)$ which is detailed in this section.

\section{Historical Background}

The need to address the limits of traditional science in developing knowledge required for nursing practice led to the development of qualitative description as a research methodology (Sandelowski, 2000; Thorne, Kirkham \& MacDonald-Emes, 1997). Within the quantitative science domain, the gold standard are experiments intended for prediction and control whereas non-experimental designs are often seen as "weak" (Sandelowski, 2000). Furthermore, the use of 
description was often considered to be the "crudest form of inquiry" in quantitative research (Thorne et al., 1997, p. 170) and the lowest rung of the quantitative methodology hierarchy (Sandelowski, 2000). Consequently, researchers followed rigid strategies to ensure data could be quantified and "reduced to mathematical probability logic" in order for descriptive research to be viewed as better data (Thorne et al., 1997, p. 170). Constrained by the positivist paradigm, the data produced through this philosophical assumption were considered "decontextualized" and “devoid of human subjectivity" by qualitative researchers (Thorne et al., 1997, p. 170). As such, the necessity to answer nursing questions regarding the human experience on health and illness led to the conception of qualitative description, an alternative to the traditional quantitative descriptive research method (Sandelowski, 2000; Thorne et al., 1997). Although the historical underpinnings of qualitative description cannot be ascribed to any one individual, Sandelowski $(2000,2010)$ put forth seminal works of the methodology and asserted its value to qualitative research in healthcare.

\section{Theoretical Foundation}

Qualitative description draws from the general principles of the naturalistic inquiry, which acts as the theoretical foundation for the methodology (Sandelowski, 2000; 2010). This orientation to inquiry asserts the commitment to studying a phenomenon in its natural state and free of artifice - to the extent that is possible within the context of the research arena (Sandelowski, 2000). More specifically, naturalistic studies aim to allow the target phenomenon to present itself as it is without any manipulation or interference with the ordinary unfolding of events while under study, leading to a "true understanding" (Colorafi \& Evans, 2016, p. 18). Additionally, the tenets of this methodology include the "recognition of varied shared experiences and the interactive-inseparable nature of human interaction" (Willis, Sullivan- 
Bolyai, Knafl, \& Cohen, 2016, p. 1193). Accordingly, following the tenets of naturalistic inquiry, qualitative description studies aim to provide a comprehensive summary of a chosen phenomenon using the everyday terms specific to the phenomenon itself (Sandelowski, 2000).

Role of Theory. Often described as the "least theoretical" of the continuum of qualitative studies, qualitative description is minimally constrained to "pre-existing theoretical and philosophical commitments" (Sandelowski, 2000, p. 337). This does not mean that qualitative descriptive studies are atheoretical or that theory does not play a significant role, but instead allows the integration of a guiding theory or framework that is most appropriate to meet the needs of the researchers' particular inquiry and chosen phenomenon (Sandelowski, 2000; 2010). As per this methodology, theory does not have to be fully pre-determined but can emerge and shift during the research process; thus, allowing for flexibility in commitment to a guiding theory or framework throughout the study (Sandelowski, 2000; 2010).

\section{Methodology Overview}

In two seminal articles, Sandelowski $(2000 ; 2010)$ described qualitative description as a distinct and well-developed, yet unacknowledged, method which provides findings in straightforward language that clearly illustrates the phenomena of interest. Qualitative description approaches are often eclectic methodologically and have the cardinal feature of supporting the use of many different theories, sampling strategies, and data collection techniques (Colorafi \& Evans, 2016; Neergaard, Olesen, Andersen \& Sondergaard, 2009; Sandelowski, 2010). The overarching goal of the approach is to describe and enhance understanding of human experiences and events that are not commonly described or sufficiently understood at a manifest level (Sandelowski, 2000; 2010; Willis et al., 2016). In particular, Sandelowski (2000) highlighted that researchers conducting qualitative description studies seek descriptive validity, 
referred to as "an accurate accounting of events that most people (including researchers and participants) observing the same event would agree is accurate", and interpretive validity, described as "an accurate accounting of the meanings participants attributed to those events that those participants would agree is accurate" (p. 336).

Sandelowski (2000) characterized qualitative description studies as less interpretative than interpretive description as described by Thorne and colleagues (1997) since findings stay closer to the data obtained. However, Sandelowski argued that "all inquiry entails description, and all description entails interpretation" (p. 335). Sandelowski further asserted that descriptions are always influenced by the describer's perceptions, sensitivities and inclinations. While qualitative description is not free of interpretation, it entails a type of interpretation that is more low-inference than studies within such traditions such as phenomenology and grounded theory (Sandelowski, 2000). It is important to note that the difference between low and high inference approaches is not one of rigour (Colorafi \& Evans, 2016), but instead refers to the amount of "conceptual or highly abstract rendering of data" required (Sandelowski, 2000, p. 335). Qualitative description encourages researchers to stay close to their data and to the surface of words and events, as opposed to other qualitative methodologies (Sandelowski, 2000). In other words, qualitative description studies offer a presentation of the chosen phenomenon in everyday language whereas phenomenological, ethnographic, and narrative descriptions "re-present events in other terms" (Sandelowski, 2000, p. 336). Accordingly, the produced findings are closer to the data as given or "data-near" (Sandelowski, 2010, p. 78), increasing the likelihood of agreement among researchers on the findings of the study (Sandelowski, 2000).

Qualitative researchers have been reluctant to use qualitative description as their choice of research approach due to their concern that this method of inquiry will present similar 
limitations to quantitative description and thus sought "epistemological credibility" (Thorne et al., 1997, p. 170) in four other methodologies: phenomenology, grounded theory, ethnography, and narrative (Sandelowski, 2000). However, Sandelowski (2000) highlighted that researchers often resort to "methodological acrobatics" as some studies that researchers labelled as one of the four methodologies could be often more accurately described as qualitative description studies with narrative, phenomenologic, and ethnographic overtones (Sandelowski, 2000). Sandelowski further asserted that qualitative description is regarded as one of the most commonly employed methodologies. This claim is further supported by Polit and Beck $(2009 ; 2014)$ in their paper which analyzed more than 1,000 nursing studies published in eight journals between 2005 to 2006. The authors found that more than half of qualitative studies were qualitative description (52\%), while the rest comprised of phenomenology (20\%), grounded theory (11\%) and ethnography studies (1\%).

Chafe (2017) expanded on the value of qualitative description by highlighting the research methodology as a well-suited approach in engaging a wide range of stakeholders. The widespread use of qualitative description has been credited to its ability to offer rich descriptions expressed in the words of the participants as well as to generate a focused summary and understanding of participants' experiences within their sociocultural context (Magilvy \& Thomas, 2009; Sandelowski, 2000; 2010; Willis et al., 2016).

\section{Rationale for Choosing Qualitative Description}

More traditional qualitative approaches (e.g. phenomenology, ethnography, and grounded theory) tend to rely on high level interpretation and theory development, moving the data away from the participant's direct experiences which are less useful for program development (Sullivan-Bolyai et al., 2005). Instead, qualitative description offers a comprehensive description 
of a chosen phenomenon using the participants' everyday language. In fact, one of the central tenets of this methodology is the commitment to collecting, analyzing and representing rich descriptions provided by participants using a process that is theoretically informed, but not theoretically bound (Sandelowski, 2000). As a result, the analysis process stays close to the data and the informants' points of view (Neergard et al., 2009; Sandelowski, 2000). Furthermore, given that this study is preliminary and exploratory in nature since the phenomenon is not wellknown, having a guiding theoretical framework was deemed inappropriate for the purposes of this study.

Despite the preliminary nature of this study, it does not mean theory did not play a significant role in its development. Qualitative description enables the integration of theory that is most appropriate to meet the needs of the chosen phenomenon (Sandelowski, 2000). The constructivist paradigm was chosen to guide the research process given that constructivism equates with theory. Indeed, theory is considered to be the "net that contains the researcher's epistemological, ontological and methodological premises" which directs the conducts of the researcher (Denzin \& Lincoln, 2005, p. 13). As such, qualitative description is the method of choice when little is known about a phenomenon and straightforward language provided by participants are needed, making data readily understood and findings interpretable to the general public (Sandelowski, 2000; Sullivan-Bolyai et al., 2005). Qualitative description is also suitable for needs assessments, especially in vulnerable populations, as well as in studies where informants' perspectives and evaluations are desired since the approach allows for the presentation of informants' points of view in their everyday language (Neergaard et al., 2009; Sullivan-Bolyai et al., 2005). 
Another benefit of using qualitative description in my study is that it averts issues associated with an academic lens which do not culturally align with the individuals experiencing the phenomenon of interest (Sullivan-Bolyai et al., 2005). In particular, qualitative description provides a platform for identifying important and constructive suggestions for intervention and program development as well as establishing interventions that are acceptable and understandable to individuals undergoing the specific phenomenon (Sullivan-Bolyai et al., 2005). Since the chosen phenomenon is poorly understood in the literature and qualitative description can identify critical information for developing new programs and interventions, this method is well-suited to understanding the experiences of family caregivers who use mobile apps in their caregiving role in order to inform future mobile app interventions.

\section{PART II: STUDY METHODS}

\section{Sampling}

In this study, I employed a purposive, criterion sampling method, in which participants were chosen based on a predetermined set of criteria (Suri, 2011). This sampling method supports the qualitative description approach as it facilitates the identification of participants who are information-rich; thereby, yielding significant insights and in-depth understanding (Sandelowski, 2000).

\section{Inclusion Criteria}

The inclusion criteria devised for the study were:

(1) Self identifies as an adult child family caregiver (i.e. child or grandchild) of a community-dwelling relative living with dementia; 
(2) Owns a mobile device (e.g. smartphones, tablets, PDAs, laptops with app features) and has prior experience using mobile applications to assist in caregiving for a relative living with dementia within the last 12 months;

(3) 18 to 55 years-old;

(4) Can read, speak, and understand English;

(5) Can provide written consent;

(6) Resides in Ontario.

The first and third inclusion criteria were informed by the following factors. First, as indicated in the literature, differences in caregiving experiences exist based on the nature of the family relationship with the care recipient (i.e. spouses versus adult children) as the two groups differ in terms of their stage of life and relationship with the care recipient. As such, this would produce differences in lifestyle, burden, associated feelings and perceptions of the situation, personal and social relationships, as well as the use of resources among different types of caregivers (Conde-Sala et al., 2010). This generational factor is important to note as better understanding of caregiving experiences of adult child caregivers can enable mobile app interventions to be tailored more effectively towards their needs. Second, with respects to mobile app usage, statistics from Canada Health Infoway show that majority of people who use mobile apps for health reasons are under 35 years old (51\%) and 35 to 54 years old (33\%). Informed by the literature and in consideration of these two factors, the first and third inclusion criteria were developed as such.

\section{Sample Size}

Constructivism stresses the uniqueness of each individual's experience and thus argues that data saturation can never truly be reached (Bradshaw, Atkinson, \& Doody, 2017). Instead, 
an adequate sample size is one that can sufficiently answer the research question by recruiting participants deemed rich in information (Bradshaw et al., 2017). When determining an appropriate sample size, three factors were considered: (1) what is recommended in user experience research; (2) the study design; and (3) the quality of the data. First, in the technology domain with respect to user experience, researchers advise a sample size of five (Nielsen \& Landauer, 1993; Nielsen, 2012; Virzi, 1992) to ten (Faulkner, 2003). Second, my study design called for the use of multiple methods and data collection points that resulted in two interviews per participant, with a total of ten interviews, conducted. Lastly, the quality of data produced in the ten interviews with the use of photo-elicitation interviewing resulted in data that are rich and experiential. Morse (2000) invites researchers to take into account parameters such as the quality of data and study design when determining sample size. Morse further posits that the more useable data (as determined by the quality of the data and the number of interviews per participant) collected from each person, the fewer participants are required. Because of these factors and after careful discussion with my thesis committee upon reviewing the quality of the data collected, I decided that the five participants recruited for this study was an adequate sample size that allowed for in-depth analysis and rich understanding of the end-user experience.

\section{Recruitment}

To gain access and recruit participants, gatekeepers (people or entities that can allow access to resources [Whicher, Miller, Dunham, \& Joffe, 2015]) in the community were contacted to disseminate study posters via email, in-person, and/or social media. They included organizations providing dementia support (i.e. Alzheimer's Society of Toronto) and advocacy (i.e. Thoughts for Dementia Collaborative), outpatient memory clinics (i.e. the Reitman Center at Mount Sinai Hospital), and online support groups for caregivers (refer to Appendix $\mathbf{F}$ for all the 
recruitment documents used in this study). Research has shown that this strategy is particularly successful because of the close relationships that referral organizations and staff have with potential participants (Szabo, Whitlatch, Orsulic-Jeras, \& Johnson, 2016). Social media recruitment strategies were also employed, such as posting in Facebook support groups and asking organizations to re-tweet or tweet recruitment notices on Twitter. However, no in-person recruitment occurred in family caregiver support groups as most organizations, such as local chapters of Alzheimer Society of Ontario, do not allow researchers to go to support group meetings for recruitment purposes to protect and respect the time of their members. On the recruitment material, potential participants were asked to contact the researcher via email if interested in participating in the study. Once a potential participant reached out to the researcher via email, the researcher either emailed or called and screened the individual for eligibility using the screening script (Appendix G).

\section{Data Collection}

Data collection in qualitative descriptive studies aims to discover "the who, what and where of events" or experiences (Sandelowski, 2000, p. 339). In this study, I employed the following data collection methods: (1) demographic form; (2) two one-on-one, semi-structured interviews with each participant using photo-elicitation interviewing in the second interview; and (3) field notes. Figure 1 outlines the data collection process that was used in this study. 


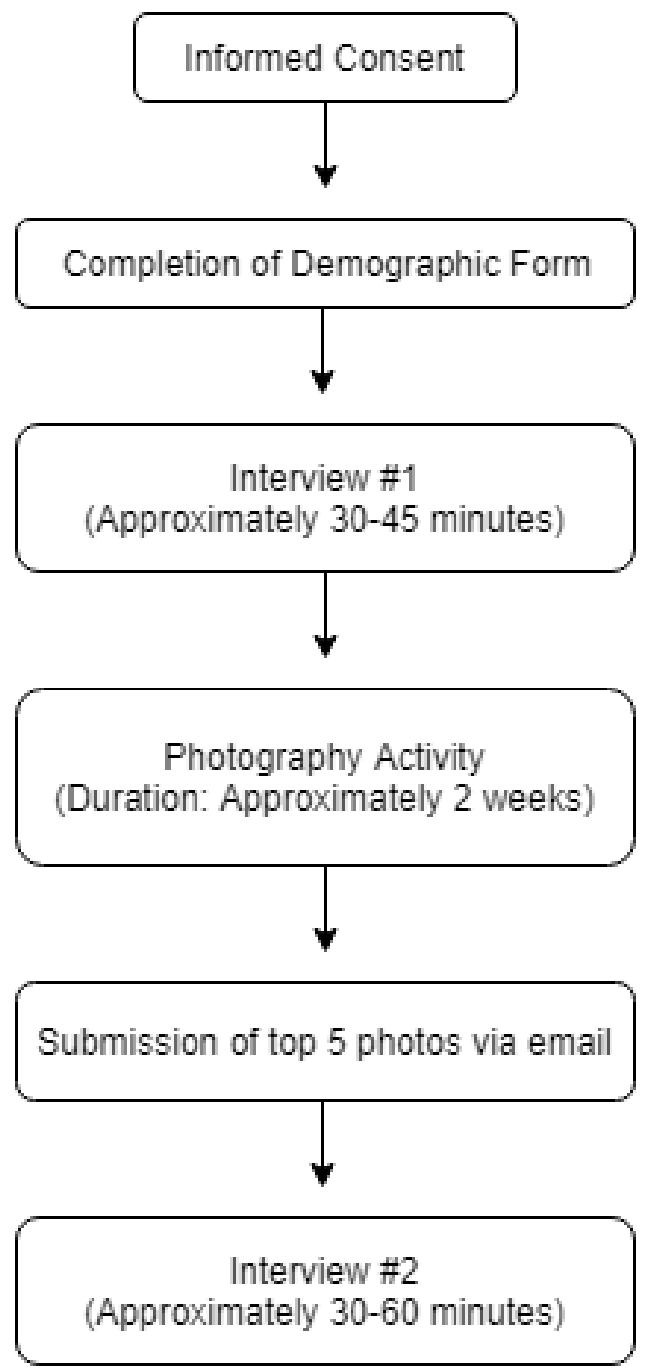

Figure 1. Overview of the data collection process employed in this study.

\section{Demographic Form}

A demographic form was used to describe the study sample. Data collected on this form included information such as: age, sex, city of residence, highest level of education, relationship with their relative living with dementia, and type of mobile device(s) the participant is using (Appendix I). American Psychological Association's (2001) Publication Manual (5 ${ }^{\text {th }}$ edition) highlights the need to provide specific information about participants' characteristics, including their educational level. The inclusion of such information is to reduce the risk of assuming the stance of "absolutism", wherein there is an assumption that the phenomenon of interest is the 
same regardless of ethnicity, culture, and educational level (Hammer, 2011). Provision of detailed information about participant characteristics promotes researchers to move toward a position of "universalism" where there is a recognition that universal psychological processes can manifest differently depending on characteristics of the participants (Hammer, 2011).

Additionally, the use of a demographic form was a method to understand certain aspects of the participant's experiences they may find difficult to immediately disclose as researchers have found that written questionnaires have been associated with low distress (Decker, Naugle, Carter-Visscher, Bell, \& Seifert, 2011). This was important to consider given that at this point in data collection, the participants and I are still in the beginning stages of building rapport and that the demographic information provided by participants will inform the interview questions. Participants were informed of the option of leaving certain areas of the form blank if they were uncomfortable answering a particular question. Ultimately, the goal of collecting the demographic data was to obtain a description of participants which allows readers and researchers to explore to whom research findings relate and allows for comparisons to be made across studies (Hammer, 2011). Furthermore, informed by the demographic data, a more comprehensive understanding of the user experiences of the study sample can be gained.

\section{Interviews}

In this study, I employed semi-structured, individual interviews as the main data collection method. Two interviews were conducted with each participant in which the second interview was informed by photo-elicitation methods. The purpose of the first interview was to gain a rich description of the participant's user experiences of using mobile apps during caregiving activities for their loved one(s) living with dementia. The first interview lasted approximately 30 to 45 minutes. At the end of the first interview, participant briefing about the 
photography activity and a $\$ 10$ gift card as a token of gratitude for their participation was provided. The second interview, which involved photo-elicitation, is detailed in the next section.

An interview guide, with examples of probing questions, was used for each of the interviews in this study (Appendix $\mathbf{J}$ and L). The interview guides were emergent and informed by previous interviews. Any new questions that arose and were added to the interview guide were discussed with the thesis committee to ensure that the questions aligned with the TriCouncil Policy Statement: Ethical Conduct for Research Involving Humans (Government of Canada, 2014) and related to the research questions. Additionally, preliminary analysis of the first interview informed the second interview. The second interview also provided the opportunity to clarify any areas from the first interview with the participant. All interviews were audio-recorded using a digital voice recorder and later transcribed verbatim by the researcher with participants' consent.

Keeping in mind that potential participants are family caregivers who may find it difficult to leave their homes due to caregiving responsibilities and/or other demands, participants were given the option of either in-person or telephone interviews. In this population, time and travel constraints are prominent; thus, offering various options is a strategy to encourage participation and decrease lower refusal rate in the sampling plan (Tao, McRoy, Kovach, \& Wang, 2016; Whitebird et al., 2011). In the study, all five participants opted to have the interviews on the phone. In the next section, I detail the photo-elicitation interviewing (PEI) process.

\section{Photo-Elicitation Interviewing (PEI)}

Photo-elicitation interviewing (PEI) refers to the use of photographs during an interview to encourage dialogue (Shell, 2014). This method was first coined by researcher and photographer, John Collier in 1957 (Harper, 2002). Based on the works of Bateson and Mead 
(1942) who were the first to use pictures as a research tool in their studies during the 1930s and 1940s (Jacknis, 1988, as cited in Shell, 2014), Collier expanded this approach by using photographs as an aid in the interviewing process. Collier described the role of photographs as a means to jog memory, stimulate emotions, and facilitate the progression of the interview in a meaningful way.

Harper (2002) contended that we, as human beings, inherently respond to images and text in different ways. Harper argued that images evoke deeper elements of human consciousness than words because the visual processing components of the brain are older than their verbal processing counterparts. As such, Harper emphasized that PEI does not simply elicit more information but evokes a different kind of information. Similarly, Genoe and Dupuis (2013) highlighted that photographs can "capture greater levels of detail about the emotional meaning of experience than words-only data collection" (p. 4). Hagedorn (1994) further elaborated on the benefits of using photography in research by emphasizing that photographs invite open expression while maintaining concrete and explicit points of reference, encourage participants to discuss their experience, and elicit a unique return of insights that may otherwise be impossible to obtain with other methods. Consequently, photographs become a medium of communication between researcher and participant with a dual purpose: (1) for researchers to use as a tool to expand on questions; and simultaneously, (2) for participants to provide a unique way to communicate dimensions of their lives and experiences (Clark-Ibáñez, 2004).

The use of photographic methods is not new in dementia research. Researchers have used this method in exploring the experiences of spousal caregivers of persons living with dementia (Aubeeluck \& Buchanan, 2006), and persons living with Alzheimer's disease (Evans, Robertson, \& Candy, 2016; Shell, 2014; Wiersma, 2011), as well as leisure in dementia care (Genoe \& 
Dupuis, 2013) and perspectives of persons living with dementia and their caregivers associated with using in-home monitoring (Epstein, Aligato, Krimmel, \& Mihailidis, 2016).

As a method that is participatory, participant-driven and collaborative in nature, PEI was chosen to supplement the qualitative descriptive methodology, to harmonize with constructivist philosophy underpinning this study, and to correspond with the user experience component of the phenomenon under investigation. The PEI framework as described by Bates and colleagues (2017) was followed in this study (Figure 2).

\section{Photo-Elicitation Interviewing Process}

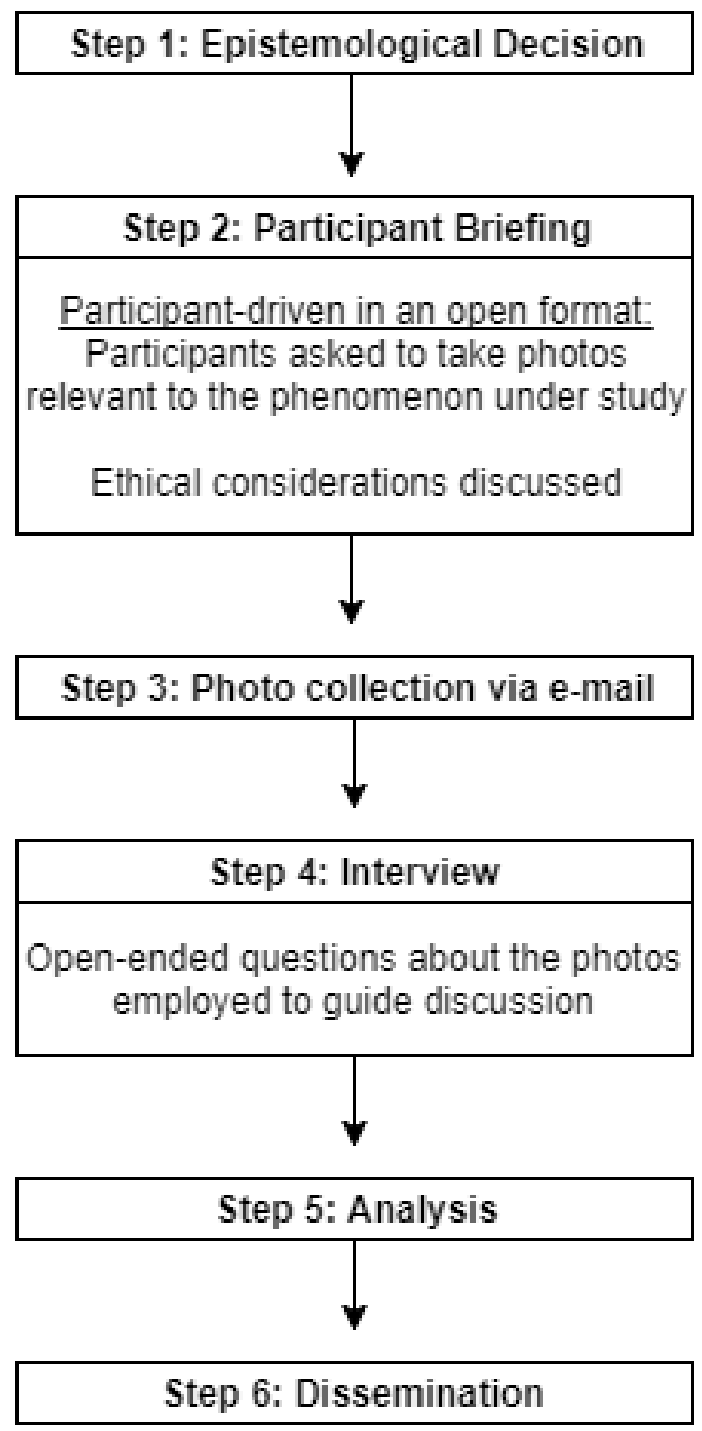


Figure 2. The photo-elicitation interviewing process for this study. Adapted from, "Beyond words": A researcher's guide to using photo elicitation in psychology, by E. A. Bates, J. J. McCann, L. K. Kaye, and J. C. Taylor, 2017, Qualitative Research in Psychology, 14(4), 474. Step 1: Epistemological Decision. Given that PEI is a flexible method in that the guidance and instructions can fit varying epistemological positions, the researcher must choose an approach reflective of their epistemological stance (Bates, McCann, Kaye, \& Taylor, 2017). Since this study is underpinned by constructivism, the inductive "auto-driven" approach of PEI was employed in the second interview wherein the photos taken by the participants guided and 'drove' the interview aimed at exploring the meanings behind their photographs (Clark-Ibáñez, 2004; Shell, 2014; Torre \& Murphy, 2015). Furthermore, the open format of the auto-driven approach was adopted, whereby participants were asked to provide any five photos they felt were relevant to the phenomenon of interest (Bates et al., 2017). This approach was suitable as it encouraged inclusion and active engagement of the participants as well as provided participants an opportunity to reflect on their experiences, resulting in the "development of meaning and insight for both researcher and participant" (Shell, 2014, p. 175). This, in turn, can increase participant-led dialogue and subsequently, rich data; bridge the world of the researcher and the participants; and facilitate rapport building (Bates et al., 2017; Genoe \& Dupuis, 2013).

Step 2: Participant Briefing. Participant briefing was provided at end of the first interview in which a detailed explanation of the photography portion of the study was provided and a copy of the information was given to the participant (Appendix K). Participants were asked to use their own camera-enabled mobile devices for photo-taking. They were asked to take at least five photographs of activities, feelings, values, and ideas that were considered meaningful and representative of their caregiving role as well as their experiences of using 
mobile apps in caregiving. Torre and Murphy (2015) contended that this approach reduces logistical issues that often arise in research involving PEI, such as unfamiliarity with other photo-taking devices. During this stage, ethical considerations were also discussed with the participant.

Ethical Considerations Associated with PEI. Ethical concerns associated with PEI include photo ownership, privacy, and consent. There was a conscientious effort in constructing an ethical research design that took consideration of these concerns. First, how the photographs are going to be used were explained to the participants. It was decided that shared ownership of the photographs taken by participants was appropriate given the participatory nature of the method (as seen in PEI studies conducted by Murray \& Nash, 2017). In terms of privacy, participants were informed that they could take pictures of people, but no defining features as it may invade the privacy of individuals who had not been consented to be part of the study (Aubeeluck \& Buchanan, 2006) such as a photograph of the participant's hand linked with another person's hand. Participants were also advised that the content of photographs should not depict anything illegal or be deemed sensitive in nature (Bates et al., 2017). Participants were told that the selected photos should not contain information that is violating any confidentiality clauses for any individual or organizations such as patient information or industry data (Bates et al., 2017). They were reminded that any photographs that violate an individual or organization's privacy will not be used in the study (Torre \& Murphy, 2015). Consent for the use of the photographs in the interviews and the use in any dissemination activities (e.g. conference presentations, publications, etc) were obtained separately in written format (Appendix $\mathbf{H}$ ) (Bates et al., 2017). 
Step 3: Photo Collection. A two-week period for the participants to collect, compile, and send their photographs to my email were allotted after the first interview.

Step 4: Interviews. Two telephone interviews were conducted for each participant and the procedures for the second interview involving PEI are outlined below:

Second Interview: Using PEI. In the second interview, the photographs served as the basis of the discussion from which I was able to extrapolate a deeper understanding and description of the participant's experiences, and later confirm through discourse (Shell, 2014). Within the interview, photos were used as a stimulus to elicit richer accounts of the participant's user experiences with mobile apps in caregiving (refer to Appendix $\mathbf{L}$ for the interview guide). The second interview lasted approximately 30 to 60 minutes. Once the interview was completed, the participant was given a $\$ 10$ gift certificate as a token of gratitude for participating in the study.

Step 5: Analysis. In the context of this study, the photographs were a conduit and catalyst for discussion at the second interview. The photographs were not coded or analyzed in this study as the associated dialogue served as the data set (Bates et al., 2017; Harper, 2002).

Step 6: Dissemination. Participants' consent was sought after for their photographs to be used in dissemination materials, such as using them in my thesis, conference presentations, journal articles and other dissemination methods. Given the purposes of this study, dissemination of the photographs in this manner can act as an effective method to bring about meaningful education and change (Dupuis et al., 2016) regarding the role of mobile apps in caregiving. Visual arts in the form of photography can provoke sensory experiences which can enable individuals to feel and see aspects of the human condition; thus, illustrating dimensions of the human experience (Lapum et al., 2016). Research has also overwhelmingly indicated that visual 
arts strategies can effectively create interest in and action around substantive areas that require change by directing critical dialogue towards social and dementia issues - something that is challenging to achieve through traditional written forms of social and dementia-related communication (Boydell et al., 2012). Thus, photographs can highlight the complexity and multidimensionality involved in creating new knowledge as well as offer alternative methods of producing and communicating research findings and best practice in healthcare (Boydell et al., 2012). Most importantly, photographs can make knowledge more accessible to diverse stakeholders and multiple audiences (Boydell et al., 2012; Dupuis et al., 2016), which is important when disseminating research findings.

\section{Field Notes}

Field notes were made during the interviewing process to enable the recording of various factors that can inform participant meaning (i.e. non-verbal behaviours such as tone of voice), promote reflexivity, and enhance "transparency of analytic insights and speculations" (Milne \& Oberle, 2005; Phillippi \& Lauderdale, 2018; Willis et al., 2016, p. 1196). The field notes helped recreate the ambience of the interview by noting the non-textual aspects of the interview. I also documented the initial perceptions and insights of each interview as well as my reflections after the interview. Given that I am an instrument within the study, shaping the findings, reflection after each interview encouraged me to assess my performance, biases, and feelings (Phillippi \& Lauderdale, 2018). Tuckett (2005) elaborated that keeping field notes contributes to the credibility of a study as these memos are "analytical in themselves" and contain "immediate and later perceptions and thoughts about the research process" (p. 32). As such, field notes are described as another data source that can contribute to credibility in the context of data triangulation (Tuckett, 2005). 


\section{Data Analysis: Thematic Analysis}

Data collected from the demographic forms were analyzed using descriptive statistical

analysis and qualitative thematic analysis were used to analyze the interviews. The qualitative descriptive approach aims to present a description of the chosen phenomenon; thus, in congruence to this approach, qualitative thematic analysis as described by Braun and Clarke (2006) was chosen. Thematic analysis is described as "a method for identifying, analysing and reporting patterns (themes) within data" (Braun \& Clarke, 2006, p. 79). Thematic analysis supports qualitative description as it is a dynamic, iterative and recursive method of analysis that promotes researchers to immerse themselves in the data and provides a detailed and nuanced account of the data (Braun \& Clarke, 2006; Vaismoradi, Turunen, \& Bondas, 2013). Thematic analysis also describes the data by adopting a low level of interpretation (Vaismoradi et al., 2013), making it congruent with the purposes of the qualitative descriptive methodology (Sandelowski, 2000). Data collection and analysis were conducted concurrently to add to the depth and quality of data analysis (Vaismoradi et al., 2013). The analysis also did not occur in a linear fashion, but instead, in a recursive manner with frequent reviews (Braun \& Clarke, 2006; Vaismoradi et al., 2013).

Braun and Clarke's (2006) thematic analysis method is an interpretive process that involves six phases of analysis which is described below.

\section{Phase 1: Familiarizing Yourself with the Data}

The aim of this phase is to become familiar with the depth and breadth of the content through immersion in the data. To achieve this, I actively engaged with the data by transcribing all interviews verbatim and reading the transcripts multiple times in an active way wherein I 
searched for meanings and patterns. Throughout this process, I also made notes of my initial thoughts and impressions prior to the formal coding process.

\section{Phase 2: Generating Initial Codes}

In this phase, I read the transcripts word by word to derive codes by first highlighting exact words from the transcript that appear to capture key thoughts or concepts. I systematically worked through the transcripts by giving full and equal attention to each line. A variety of methods through Microsoft Word were used to illuminate data segments or codes into meaningful groups, such as multi-coloured digital text highlighting and comment boxes. I coded as many patterns as possible to ensure every meaningful segment of data is identified. I coded extracts of data to ensure that the context is included. I also approached the text by making notes of my first impressions, thoughts, and initial analysis using comment boxes on the side of the margin of the document.

\section{Phase 3: Searching for Themes}

Once all the transcripts were initially coded and collated, I began to sort the different codes into potential themes. To accomplish this, I collated all the codes into a separate Microsoft Word document in a table format to organize similar codes together. Throughout this process, I analyzed the codes and how relevant coded data extracts may combine to form a broader, overarching theme. Visual representations were used to help sort the different codes into themes by using tables and diagrams. A preliminary thematic map was developed after analyzing the connection and relationship between codes, between themes, and between different levels of themes. In this phase, a collection of main themes and subthemes was created. A section titled "miscellaneous" was used to house codes that do not seem to fit into the main themes. 


\section{Phase 4: Reviewing Themes}

The main themes and subthemes created in the previous phase were reviewed, revised and refined in this phase. In order to do so, internal homogeneity and external heterogeneity were used to evaluate main themes and subthemes. This was done by ensuring the data within themes should cohere together meaningfully, while clear and identifiable distinctions between themes also exist. Afterwards, necessary changes to the thematic map and organization were made to ensure data within themes are consistent and form a coherent pattern while themes are distinct from each other. To enhance trustworthiness, the codes, subthemes, and themes were reflected on and discussed with the thesis committee throughout the analysis process, resulting in consolidation of the findings. In any instances of incongruence, further analysis was conducted to evaluate whether the theme is not appropriate or the data extracts within the theme do not fit. After analysis, I either reworked the theme, created a new appropriate theme, moved the data extracts that do not currently work into another main theme, or removed it from analysis. Segments of data were rearranged within themes until they are contained within a relevant theme. Lastly, the appropriateness and validity of individual themes were considered in relation to the complete data set to see whether the thematic map accurately reflects what is portrayed in the whole data set. The data set was reviewed in its entirety to ensure if the main themes and subthemes fit with the whole of the data.

\section{Phase 5: Defining and Naming Themes}

In this phase, the main themes and subthemes were defined and further refined as I considered the essence of what each theme is about and organized data extracts into a coherent and internally consistent narrative. During refinement, I kept in mind any possible overlap between themes and how each theme related to other themes. Themes were checked if they are 
internally coherent, consistent and distinctive. I also considered how each theme connects to the data as a whole and how it fits into the broader overall 'story' of the complete data set. Once clear definitions for each theme were generated, each main theme and subtheme was given a "concise, punchy" name to immediately give the reader a sense of what the theme reflects (Braun \& Clarke, 2006, p. 93).

\section{Phase 6: Producing the Report}

In this final phase, vivid, compelling extract examples were selected to provide sufficient evidence of the themes within the data and to capture the essence of the story the data tells. An analytic narrative was written to illustrate the story the data tells in relation to the research questions with illustrative extract examples embedded within.

\section{Ethical Considerations}

The following three principles provided the organizing framework regarding ethical considerations for this study: (1) beneficence, referring to the notion that participants must not be harmed; (2) autonomy, pertaining to the fact that informed consent must be obtained and participation must be voluntary; and (3) justice, regarding the need for researchers to uphold participant confidentiality and anonymity as well as to treat participants with dignity and respect (Streubert \& Carpenter, 2013). To ensure informed consent, participants were given adequate information regarding the study and the researcher's role as well as told that their participation is completely voluntary, and they are free to withdraw at any time without penalty (Streubert \& Carpenter, 2013). Participants were provided the consent form at least a week before the scheduled interview through email. They had the opportunity to review and ask any questions relating to the consent form. On the scheduled day of the interview, I reviewed the consent form with the participants again and answered any additional questions. They were reminded that 
participation is completely voluntary and that they could leave the study at any time without consequences. Written consent for the interview, audio-recording, and the use of photographs taken by participants in dissemination materials, were obtained before conducting the interview through email. Participants also received a copy of the consent form. Process informed consent was employed, where at varying points in the research process, participants' consent to participate was re-evaluated (Streubert \& Carpenter, 2013). No participants withdrew consent in the study. However, it was explained to participants that in the case should a participant withdraw consent, they would not be participating in subsequent study activities; and they may also request to have previously obtained information removed prior to when data analysis will take place.

Participants were instructed that they do not have to provide a response for any questions they do not feel comfortable answering. Strategies used in this study to ensure participant confidentiality and anonymity included storing all data in a secure locked location, removing identifier components, and assigning participants a study ID number (Sanjari et al., 2014). Recordings of the interviews were destroyed right after transcription. All electronic data were stored using password-protection and encryption. In addition to myself, only members of the thesis committee had access to study data. Ethics approval was obtained from Ryerson University's Research Ethics Board prior to the commencement of the study. All data will be destroyed one year after the publication of my thesis in case any audits arise after publication.

\section{PART III: REFLEXIVITY AND TRUSTWORTHINESS}

\section{Reflexivity: "Researcher as an Instrument"}

Reflexivity requires researchers to make their position explicit by being conscious of the biases, values, and experiences that they bring to the study and by describing their existing 
beliefs and assumptions early in the research process as a means to make one's positioning overt to self and readers (Creswell, 2013; Creswell \& Miller, 2000). Since researchers are described as the primary data-gathering instruments in qualitative research (Lincoln \& Guba, 1985), Primeau (2003) asserted that "reflexivity enhances the quality of research through its ability to extend our understanding of how our positions and interest as researchers affect all stages of the research process" (p. 10). In other words, this allows readers to understand the positions of the researcher (Creswell \& Miller, 2000). This form of reflexivity was incorporated into the prologue in a narrative account.

Field notes, which consisted of reflective memos and a research diary, were also kept to foster reflexivity in this study. The research diary promoted ongoing self-critique and a "selfcritical account of the research process" (Rolfe, 2006; Tobin \& Begley, 2004, p. 392). In the research diary, I recorded my decision-making processes, what influenced my interpretation of the data as well as my relationship to the research topic and the participants (Jootun, McGhee, \& Marland, 2009). The interviews were recorded and transcribed by myself in order to gain awareness of the subtle ways in which I may have been influencing the data collection (Jootun et al., 2009).

\section{Trustworthiness: Establishing Rigour in Qualitative Research}

Building on the seminal works of Guba and Lincoln (1981), Sandelowski's $(1986,1993)$ framework for establishing rigour was chosen to guide this study to maintain congruency to the chosen methodology of qualitative description as described by Sandelowski (2000). While I acknowledge that additional perspectives and frameworks exist for establishing rigour in qualitative research, the decision to use the framework described by Sandelowski (2000) was a result of a thoughtful process. First, consultation was sought from two Master of Nursing 
graduates from Ryerson University who used the same methodology and rigour framework for their thesis work. They explained that maintaining congruency throughout their study was important. Second, the paradigm assumptions underlying the research should govern the choice of rigour procedures (Creswell \& Miller, 2000). Thus, certain typologies of rigour were deemed inappropriate not only for the methodology employed, but also the paradigm underpinning the study given the framework's philosophical and theoretical assumptions. Last, I also took in consideration that I was implementing a methodology described by Sandelowski (2000) in this study and the fact that she is a researcher in the nursing context. Because of those three main reasons, I felt that using her framework for establishing rigour was appropriate and well-suited. Sandelowski’s (1986) conceptualization presented four factors in establishing methodological trustworthiness in qualitative research: credibility, fittingness, auditability, and confirmability. Additionally, the strategies I chose to ensure trustworthiness were informed by my philosophical perspectives which are discussed further throughout the next section.

\section{Credibility}

Credibility in qualitative inquiry is "subject-oriented rather than researcher-defined" as the truth value of the research is found in discovery of human experiences as they are lived and perceived by participants instead of verification of a priori conceptions of such experiences (Sandelowski, 1986, p. 30). As such, five strategies were used to foster credibility which reflects my epistemological assumptions wherein I aim to become immersed in the data, lessen the distance between researcher and participants, and study participants in their natural setting. First, I established a close researcher-participant relationship to increase direct access to the participant's experiences, but maintained an appropriate distance for personal reflections (Sandelowski, 1986). Second, I ensured participant-driven data by probing for depth during 
interviews to promote richness (Milne \& Oberle, 2005). Third, I obtained validation from the participants themselves (Sandelowski, 1986) by probing to clarify what participants said during the interviews; summarizing the major points at the end; as well as giving participants the opportunity to reflect on the discussion while still in the context of the interview and afterwards in the follow-up, second interview to indicate if they agree with the summary provided (Milne $\&$ Oberle, 2005). Fourth, throughout the research process, I shared my way of thinking, ideas, and interpretations with my thesis committee for their feedback and questioning. I presented and justified my analysis and continuously illustrated how the study findings were grounded in the data. Last, I triangulated across data sources and data collection methods (i.e. interviews, photographs and field notes) to evaluate the congruence of findings among them (Sandelowski, 1986).

\section{Fittingness}

Fittingness is achieved when the findings fit into contexts externally from the study and are meaningful to another group (Sandelowski, 1986). Additionally, fittingness also refers to findings fitting the data from which they are derived and grounded in the experiences of participants studied (Sandelowski, 1986). Fittingness was attained in this study by using the following three methods: (1) using purposive sampling to ensure participant representativeness of the phenomenon; (2) iteratively checking for representativeness of data in the data analysis process (e.g. representativeness in the themes and examples used to reduce and present the data); and (3) richly describing the data, the typical and atypical elements, and the contexts in which the phenomenon was experienced by participants (Sandelowski, 1986). Overall, fittingness was achieved by providing an accurate and rich description of the research findings. 


\section{Auditability}

Auditability is established when the "decision trail" used by the researcher is made transparent so others could follow and understand the decision-making process (Sandelowski, 1986, p. 33). In this study, a clear decision trail from its beginning to its end is available for external perusal by richly describing, explaining, and justifying each component of the research process in detail (Sandelowski, 1986). For example, in terms of data analysis, data display charts indicating coding procedures and the process of grouping data into themes is available. An audit trail of decisions and personal reflections were also preserved in memos through the course of the study in the form of field notes and the research diary (Willis et al., 2016).

\section{Confirmability}

Confirmability was attained in this study by establishing credibility, fittingness, and auditability as well as by valuing subjectivity and seeking for truth by interacting with, rather than disengaging from, the phenomenon being investigated (Sandelowski, 1986). Additional strategies that were used to ensure confirmability include a description of the demographics of the participants as well as findings representing the data gathered, as evidenced by the inclusion of direct quotes from participants (Bradshaw et al., 2017). Most importantly, to reflect my axiological assumptions of positioning myself in the study, confirmability was addressed through awareness and reporting of personal assumptions and potential bias in the prologue (Colorafi \& Evans, 2016). My biases, assumptions, and positioning were considered throughout the research process; particularly as I reflect on my analytic and interpretive insights when collecting and analyzing the data. 


\section{CHAPTER FIVE \\ STUDY FINDINGS}


"In photography there are no shadows that cannot be illuminated."

- August Sander

(German Portrait and Documentary Photographer)

\section{INTRODUCTION}

In this chapter, I begin with a general overview of the participants in the study. This is followed by a detailed description of the study findings in two sections. In the first section, the findings addressing the main research question and the following sub-questions are presented:

(1) What are the user experiences of family caregivers using mobile apps to assist in caregiving for their loved ones living with dementia in the community? (main research question); (2) What are family caregivers' perspectives on the use of mobile apps to support caregiving activities?;

(3) What are the influences that impact the use of mobile apps for family caregivers?; (4) How do family caregivers use mobile apps during their caregiving activities? In the second section, the findings addressing the following research sub-question pertaining to the study methods are discussed: What are family caregivers' experiences of using photographs as a conduit for explicating their user experiences?

Throughout the presentation of the main themes and their affiliated subthemes, specific participant quotes and participant-captured photographs have been selected to exemplify and to describe each theme and subtheme. In order to provide a balanced representation of the participants, the selected quotes and photographs fairly represent the experiences of each participant. Of note, each photograph captured by the participant is named after a poignant or meaningful segment of the discourse that they attributed to the photograph. This was done to further highlight the participant's experiences in their own words, and to stay closer to the data; thus, aligning with the chosen methodology (Sandelowski, 2000; 2010). 


\section{THE PARTICIPANTS}

All five participants identified as female and between the ages of 18 to 35 years old. They identified themselves as a family adult child caregiver to either a parent or grandparent living with dementia. As per the specified inclusion criteria, all the participants identified as living in Ontario and having used mobile apps in their caregiving role within the last 12 months. To safeguard the identities of the participants, pseudonyms were assigned to each participant. The process of selecting the pseudonyms was a deliberate process as I chose names that reflect the emotions and feelings I had towards each participant's narratives.

Participant One was named Abigail which is derived from a Hebrew name meaning "my father is joy" (Behind the Names, 2018). I chose this name because she has a father living with Young Onset Dementia; and from her stories, the strength of their relationship with each other shined through. In particular, the story of how her father learned to use a mobile app to order a cake for her birthday spoke volumes of their bond with each other. Abigail recently graduated from university and is currently working full-time. She lives in the same household as her father and her mother. She acts as the primary caregiver for her father along with her mother. Her siblings currently reside outside of the country. She uses the following platforms to access mobile apps: her iPhone and MacBook Pro.

I chose to give the name Cassandra for Participant Two because it is derived from a Greek name meaning "to excel or to shine" (Behind the Names, 2017a). What had the biggest impact on me during our discussions together was Cassandra's reflection upon how she felt that she lacked the skills and knowledge for care provision when she compared herself to her aunt, who is a Registered Nurse. Despite feeling this way, she stated that she could still excel and shine as a caregiver if she did the best that she could within her abilities. Cassandra has a 
paternal grandmother living with dementia who lives with her aunt, the primary caregiver.

Cassandra is currently enrolled full-time in university. She visits her grandmother regularly, usually on the weekends. The platforms that she uses to access mobile apps are her iPhone and her grandmother's iPad.

Participant Three was given the name Sierra which is the Spanish word for "mountain range" (Behind the Names, 2019a). For the study, Sierra took a photograph of trees, leaves and branches that looked like somewhere obscure to symbolize the feelings of confusion, chaos and isolation associated with being a long-distance caregiver. Of note, Sierra was distinct from other participants as she was the only one who resided in another city from the care recipient during the time of the study. For me, that photograph not only vividly illustrated her feelings, but also helped me to better understand her experiences. As such, congruent to her metaphor using nature, I chose a name that represented those feelings of distance and remoteness in the form of a mountain. Sierra has a mother living with Young Onset Dementia who requires considerable assistance with her activities of daily living due to the stage of her condition. Her mother lives with her father and siblings in the same household. They have access to homecare where personal support workers visit them twice a day to help with care. Sierra is currently enrolled full-time in university. Given that her university is in a different city from home, she lives on campus and visits her mother regularly, usually during weekends. She accesses her iPhone and MacBook to use mobile apps.

Nadia was the name I chose for Participant Four due to its meaning of "hope" in many Slavic languages (Behind the Names, 2019b). The importance of hope was a salient element of Nadia's story of caregiving for her maternal grandmother living with dementia. Majority of her mobile apps use in her caregiving role was to bond with her grandmother through music played 
on those apps. What stood out to me the most was Nadia's story of how music helped her grandmother when she was hospitalized and critically ill; thus, Nadia described music as being her grandmother's hope. Nadia's grandmother lives alone in her own apartment. Her grandmother also possesses her own business and has been described by Nadia as relatively independent with her activities of daily living. According to Nadia, her mother acts as the primary caregiver for her grandmother. She recently graduated from university and is currently working full-time in the healthcare field. She accesses mobile apps on her iPhone, iPad and laptop.

Lastly, I chose to give Participant Five the name Sophia as it means "wisdom" in Greek (Behind the Name, 2017b). Through our discussions, I came to learn that Sophia had a wealth of experience and knowledge regarding caregiving as she has been a caregiver for all of her grandparents for many years. As the oldest participant and the one who has been a family caregiver the longest, I felt the name Sophia, referring to wisdom, was the most fitting. Sophia has a maternal grandfather living with dementia who lived with her grandmother in the same household. She graduated from university a couple of years ago and is also currently working full-time in the healthcare field. Her android phone, android tablet, laptop and smartwatch are the platforms to which she accesses mobile apps.

A general overview of participant characteristics is provided in Table $\mathbf{1}$ based on what participants reported in the demographic form as well as my interactions with them during the data collection process. 
Table 1. Demographic data results

\begin{tabular}{|c|c|c|c|c|c|}
\hline Participant & Abigail & Cassandra & Sierra & Nadia & Sophia \\
\hline Age & $\begin{array}{l}22 \text { to } 25 \\
\text { years-old }\end{array}$ & $\begin{array}{l}18 \text { to } 21 \\
\text { years-old }\end{array}$ & $\begin{array}{l}22 \text { to } 25 \\
\text { years-old }\end{array}$ & $\begin{array}{l}22 \text { to } 25 \\
\text { years-old }\end{array}$ & $\begin{array}{l}30 \text { to } 35 \\
\text { years-old }\end{array}$ \\
\hline Gender & Female & Female & Female & Female & Female \\
\hline $\begin{array}{l}\text { City of } \\
\text { Residence }\end{array}$ & Toronto & Toronto & Waterloo & Toronto & Toronto \\
\hline $\begin{array}{l}\text { Highest } \\
\text { Level of } \\
\text { Education }\end{array}$ & $\begin{array}{l}\text { Post- } \\
\text { secondary }\end{array}$ & $\begin{array}{l}\text { High School } \\
\text { Diploma }\end{array}$ & $\begin{array}{l}\text { High School } \\
\text { Diploma }\end{array}$ & $\begin{array}{l}\text { Post- } \\
\text { secondary }\end{array}$ & $\begin{array}{l}\text { Post- } \\
\text { secondary }\end{array}$ \\
\hline $\begin{array}{l}\text { Relationship } \\
\text { with Care } \\
\text { Recipient }\end{array}$ & Child & Grandchild & Child & Grandchild & Grandchild \\
\hline $\begin{array}{l}\text { Gender of } \\
\text { Care } \\
\text { Recipient }\end{array}$ & Male & Female & Female & Female & Male \\
\hline $\begin{array}{l}\text { Platforms } \\
\text { Used to } \\
\text { Access } \\
\text { Mobile Apps }\end{array}$ & $\begin{array}{l}\text { iPhone, } \\
\text { MacBook Pro }\end{array}$ & iPhone, iPad & $\begin{array}{l}\text { iPhone, } \\
\text { MacBook }\end{array}$ & $\begin{array}{l}\text { iPhone, iPad, } \\
\text { laptop }\end{array}$ & $\begin{array}{l}\text { Android } \\
\text { phone, } \\
\text { Android } \\
\text { tablet, laptop, } \\
\text { smartwatch }\end{array}$ \\
\hline
\end{tabular}

Given that a generation is a group of individuals who are in the same age range and have experienced similar events, their similar experiences often influence their views of the world (Statistics Canada, 2012). Hence, in order to better understand the context of the participants' experiences with technology overall, this section explicates the characteristics of the generations in which the participants grew up in. In this study, all the participants were between the ages of 18 to 35 years old. Thus, they belonged to either Generation Y/Millennials (born between 1977 and 1995; 24 to 42 years of age) or Generation Z/Centennials (born 1996 and later; 23 years old and younger) (Karr, 2017). These generations are characterized by rapid technological changes wherein they grew up in a technology-filled and online-driven society (Karr, 2017; Statistics Canada, 2012). In Abacus Data's 2018 Canadian Millennials Report, survey results showed that 
people in the Millennial generation's coming of age experience was influenced heavily by technology (Kishchuk, 2018). For instance, the average age millennials began using a computer was at 12 years-old and began adopting laptops and smartphones as early as 13 years-old (Kishchuk, 2018). Compared to other generations, such as Baby Boomers (born 1946 to 1964) and Generation X (born 1965 to 1976), participants in this study who are part of either the Millennials or Centennials generation are more likely to own and use mobile devices and accessories, including smartphones, smartwatches, and tablets, as a result of growing up in a technology-driven society (Karr, 2017).

\section{PART I: USER EXPERIENCES OF FAMILY CAREGIVERS}

In this section, the findings presented address the main research question as well as the research sub-questions related to user experiences of mobile apps. First, the overarching theme that arose from the data, Connecting to support through mobile apps in my, your, and our lives, is described in detail. Afterwards, the three main themes and their affiliated subthemes considered central to the user experiences of family caregivers are discussed. These three main themes are (1) Adapting mobile apps to meet individual needs of the dyad; (2) Minimizing the impact of the condition on the person and the family; and (3) Determining the effectiveness of mobile apps. The following figure is a visual presentation of the three main themes and their affiliated subthemes, which represents the participants' experience with using mobile apps in their caregiving role as a whole (Figure 3). 
Figure 3. Visual presentation of the study findings.

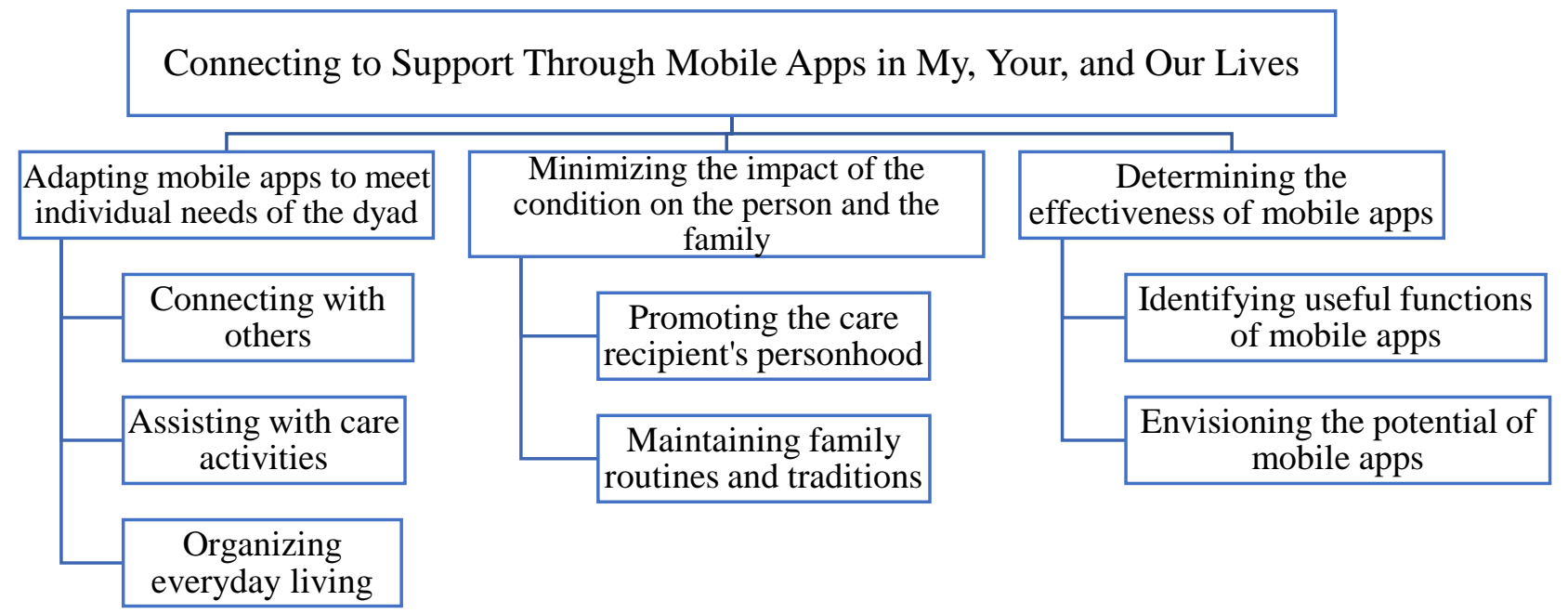

\section{Overarching Theme:}

\section{Connecting to Support Through Mobile Apps in My, Your, and Our Lives.}

While each participant's app use depended upon individual reasons and individual contexts, all participants turned to mobile apps for support on the various challenges they faced in their caregiving role, as well as in their day-to-day life. Accordingly, this theme is identified as the predominant, overarching theme and is evident across all of the three major themes. The overarching theme describes how mobile apps play an important role in the lives of the caregiver, the care recipient, and both of them together as a dyad. In other words, the role of mobile apps can shift from meeting the individual needs of the caregiver, to the care recipient, or to them as a dyad. The theme also emphasizes how the impact of mobile apps for both the caregiver and care recipient are inextricably linked as the use of apps by family caregivers can indirectly benefit the lives of the relatives living with dementia, and vice versa.

The use of the word "connect" in this theme is purposeful to highlight the user experiences of participants. "Connect" has multiple meanings and connotations in regard to 
relationships and technology which both played an important role for participants. In terms of relationships, mobile apps supported participants to connect - to link, to feel an affinity, to join together - with their care recipient in various ways to promote personhood, to facilitate displays of love and affection, and to strengthen their relationship with the care recipient. At the same time, in the technology domain, "connect" or "a connection" refers to the successful completion of necessary arrangements so that two or more entities (either programs, systems, or people) can communicate or transfer information at a distance to meet a certain goal or task (Rouse, 2005). At the root of both uses of the word is the fundamental concept of coming together and forming a relationship which is of particular significance to the participants when describing their user experiences with mobile apps. In addition, the word connect is denoted with a suffix of -ing to demonstrate that it is a present participle which means it is currently happening. This is done to reflect how participants are still actively using mobile apps in their caregiving roles and that it is an ongoing act. As such, the other main themes and its subthemes also begin with a present participle to closely align with the participants' user experiences.

\section{Main Theme \#1: Adapting Mobile Apps to Meet Individual Needs of the Dyad}

Caregivers often adapt available mobile apps to meet the needs of both themselves and the care recipient as many apps are not specifically developed for caregiving purposes. Of note, available mobile apps are mostly for commercial use - designed and developed without caregiver involvement. Consequently, the mobile apps participants use are ones that they have adapted to address their caregiving needs, as saliently illustrated by Sierra: "I feel like if you're a caregiver, you are used to adapting to everything, right?” Accounts of mobile app use predominantly focused on the benefits they brought to not only the participants and their caregiving roles, but also to the care recipient as reflected in three subthemes. In the first 
subtheme, Connecting with others, participants described the various ways they used mobile apps to communicate and connect with family members, friends, and other caregivers around the world. Through effective communication enabled by the use of mobile apps, participants were able to coordinate care among family members and assist with care activities more efficiently. In the second subtheme, Assisting with care activities, participants shared how they used mobile apps to help with certain care activities that they perform regularly as a caregiver. In the last subtheme, Organizing everyday living, participants discussed using mobile apps to help not only organize their caregiving activities, but also other facets of their everyday life beyond caregiving such as school, personal lives, and work commitments.

\section{Subtheme \#1: Connecting with Others}

Participants identified communicating and connecting with others as a crucial element in their caregiving experiences. As such, having various mobile apps that enabled participants to connect with others in varying ways was perceived as essential to them as a caregiver. Specifically, through mobile apps, participants were able to garner social support from family members, friends and other caregivers in a convenient and accessible manner. In doing so, mobile apps were able to fulfill their caregiving need of building a connection with other people, as illustrated by a statement made by Sierra:

I think a lot of them [apps] fulfill connection so my connection especially when I am away from home, I am able to connect in with my family but also connect with other caregivers and people I don't know and my peers and help them understand the situation.

Family members were not only viewed as an integral part of the caregiving team, but also seen as a critical source of social support for participants. For this reason, all participants were keen to use communication apps, such as FaceTime ${ }^{\mathrm{TM}}$, WhatsApp ${ }^{\mathrm{TM}}$, and WeChat ${ }^{\mathrm{TM}}$, to keep in contact and to coordinate care among family members. Particularly, participants emphasized the 
importance of effective communication among family members when it comes to caregiving. For instance, Sophia noted, "We have to call each other and communicate with each other".

Therefore, the availability of communication apps to help participants keep in contact with family members and the care recipient was especially valuable when they are apart from them and/or caregiving from a long distance. Abigail noted the use of WhatsApp ${ }^{\mathrm{TM}}$ and Facebook Messenger ${ }^{\mathrm{TM}}$ to communicate with her sisters who reside outside of the country: "It helps very much, especially with my sisters because I talk to them almost every day and I give them updates about my dad too on a weekly basis... WhatsApp, Facebook Messenger, these two are used very often.” For Cassandra, she would assist her grandmother in using FaceTime ${ }^{\mathrm{TM}}$ to connect with other relatives: "She uses FaceTime - well, I help her use FaceTime whenever she wants talk to my dad, or other family members." Similarly, Sophia would use WeChat ${ }^{\mathrm{TM}}$, a Chinese communication app, to not only let her, but also her grandfather living with dementia connect with other relatives across the world: "Sometimes we do WeChat with my aunt in China. We would call her and stuff like that. We would WeChat. Also have a cousin in New Zealand and we would WeChat with him too so that he would see the grandparents and stuff." Additionally, as a caregiver who is away from home for university, Sierra described using an app that allowed for long-distance phone calls through her smartphone using her landline phone. This helped reduce the financial burdens associated with making long-distance calls through a smartphone:

The easiest thing I can think right now is text messaging to keep in contact with my family especially like I am caregiving away from home as well. And just checking in and being able to talk to them is really important. I also have this app that that allows me to make long-distance phone calls but using like our landline phone instead of my cell phone since it will be a larger charge... Since we already have a landline with long distance minutes versus on my phone, I don't have that on my plan so that's really helpful because it's easy for me to follow, to talk to my dad or my sister. 
Participants also evaluated that the forms of communication apps they used (e.g.

FaceTime $^{\mathrm{TM}}$ ) allowed for conversations that are rich in social cues as it enabled participants to see the care recipient's face and vice versa. Communication apps offered many of the characteristics of in-person, face-to-face communication, such as providing visual and auditory cues. Ultimately, these apps promoted the ability to have face-to-face social contact in a virtual manner not afforded through an audio-only phone call. Participants felt that this was important when connecting with their loved ones living with dementia as it enabled them to see social cues as well as non-verbal behaviours and facial expressions. For instance, Nadia appreciated the ability to have a virtual face-to-face conversation with her grandmother through the use of FaceTime $^{\mathrm{TM}}$ as it promoted authenticity, immersion and a sense of presence similar to being in the same room:

When my brother and I were in university. We definitely would use it because I would FaceTime my parents all the time and everything. So yeah sometimes she would be in the background. And she would be so excited, like she couldn't believe that she could see my face and talking to me. Because she is not quite used to that. She forgets that you could do that. So everytime I would FaceTime her again, she would get really excited... Yeah, I think it helps. It helps me, you feel like you're actually in the room with the person that I'm not just listening to their voice, I can see their facial expressions or their little mannerisms that you might miss when you are just on the phone with someone so yeah, I think it's a lot nicer, it connects you both a little better.

Sierra described similar sentiments of the significance of being able to give and receive visual cues, as she asserted the value of using FaceTime ${ }^{\mathrm{TM}}$ to connect with her mother living with dementia:

And I obviously use FaceTime to talk to my siblings. We used a lot too in the beginning to talk to my mom since she would ask them for me. She was able to see my face and talk to me which would help because sometimes she would be confused about where I was so that is a big one.

Not only did the use of communication apps provide reassurance and comfort to the care recipient, but there was also a reciprocal component. Participants were also reassured when they 
were able to see for themselves that their family member living with dementia was safe, supported, and taken care of. This was especially prominent when participants were caregiving from a long distance and did not live in the same household as the care recipient. For instance, Sierra explained that using communication apps like FaceTime ${ }^{\mathrm{TM}}$ gave her a sense of connectedness and closeness to her mother and the opportunity to see how she is doing in a convenient manner:

I know that I have said that a million times already but being away from home is difficult so that form of technology really helps stay connected and know what's going on at home. It makes it more convenient to kind of check in because I could also do a video call or something like that. So I can actually see what's going on and see my mom and see how she is and talk to her in a more personal way instead of just over the phone because she like doesn't understand the phone that well.

Moreover, Sierra further stated that a lack of communication throughout the family was described to be a substantial issue, as it can cause confusion, chaos and stress. By overcoming certain barriers to communication through the use of mobile apps, Sierra felt that it minimized any miscommunication and reduced the feeling of being distant from the care recipient and her family. The significance of connecting to her mother and her family through mobile apps was encapsulated by the following photograph and the additional comments shared by Sierra: 


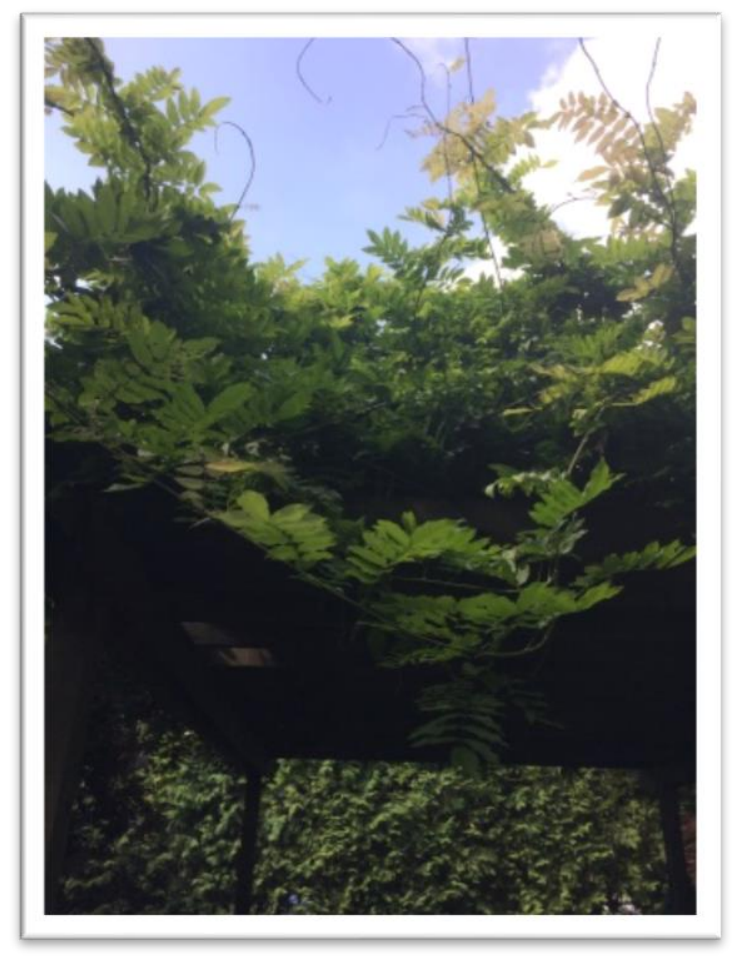

Photo 1. "Technology allows me to be still connected to my family and lessen that gap between here and there" - Taken by Sierra.

Kind of to portray like caregiving from afar because it is like you don't really know what's going on. It's like everything is kind of chaotic in the picture like the leaves are everywhere and stuff. But also, it also feels like a whole like other world away. And then, what I was also thinking is like the way that like technology allows me to feel, to be still connected to my family and like kind of lessen that gap between like here and there. But the photo was mostly to portray like how different of a place that it feels sometimes - the disconnect between communication sometimes, like my family, like being able to connect with them which is very hard because they are very busy caregiving and like work and everything that it's sometimes hard to maintain communication. So sometimes, I feel like I don't know what is going on when I am home, like when I am in [city], I don't really know what is going on at home. I have to wait until I come back to kind of be caught up on how my mom is doing and stuff like that... Like FaceTime, it kind of helps with that like disconnect and feeling like a whole different world because you can actually like see the person so like having that, it's almost like a face to face conversation and I feel like sometimes, it's like more pressing - then like a phone call if you can see them. It kind of helps to lessen that and it is also because it is like face to face, you can see people's like reaction and everything. And it can help with that miscommunication which can lead to like chaos and everything. 
Communication and social media apps also enabled the care recipient to stay connected with others. Relational interactions were identified by participants to be crucial toward helping their loved one living with dementia feel a sense of connectedness to others and the world. For instance, Nadia described using these apps to prevent social isolation for her grandmother as her condition limited her ability to connect with others in person. Therefore, Nadia's family would not only use FaceTime ${ }^{\mathrm{TM}}$ to connect with each other, but also the Facebook ${ }^{\mathrm{TM}}$ app to let Nadia's grandmother connect with their family friends who lived far away. Nadia's family achieved this by helping her grandmother view photos of family friends as she is unable to travel that far to visit in person. Nadia presented the following photograph of her iPad to emphasize how it is used on a regular basis to help her grandmother feel connected to others:

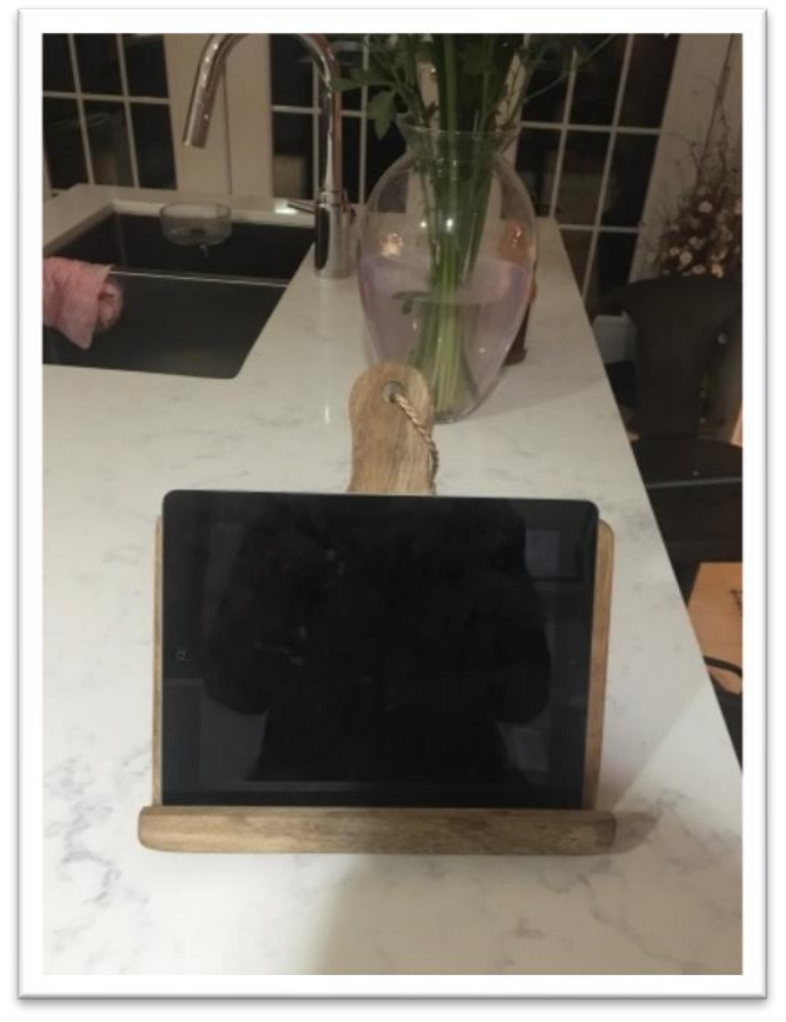

Photo 2. "Staying connected" - Taken by Nadia.

The iPad is usually what my mom would use with her to FaceTime my brother and I at school or to listen to music every time she is over. We would use that because it's a 
bigger screen than our phones. So we can show her what's on there. We'll show her pictures and things from past vacations that we've been on and graduation photos, stuff like that. So we use that with her a lot... Sometimes, my mom will go on the Facebook app and show her pictures. So just context, background: we have a cottage up on [location name] and it's an island and they have a bunch of friends that are up there and my mom and my grandma has known them forever like their kids grew up together kind of thing. So my mom will show from Facebook pictures of them from the island. So like that they can connect sort of because my grandma won't see them, she doesn't like to come up to the cottage too much because it's far away. It's not that comfortable to be away from home and whatnot. So my mom will show her pictures of friends and people that she hasn't seen for a while. Yeah, that's good, staying connected.

In addition to keeping connected with family members and the care recipient, one participant was also keen to use social media apps as a way to connect with peers and other caregivers. Sierra stated that she would share her experiences as a caregiver on social media apps in the form of pictures and videos to promote dialogue among peers and to help them better understand what her experiences are as a caregiver of a person living with dementia:

I use like social media: Facebook, Twitter, mostly Instagram to share pictures and it's like, not something that my peers can relate to but when I share a picture of my mom and have a little caption - it's easier for them to understand I find. It's a better way to have that conversation because I'm the one that is opening up and starting like the dialogue there so that's been really helpful, a way for me to connect with my peers.

In this context, the use of social media apps worked as a way of finding individuals in similar circumstances. Sierra further explained the value in having a support system consisted of people who understand what she is going through: “...connecting with other people and how having that kind of support system is important and there are people that understand, you don't have like to explain everything to." This in turn helped her gain social support from other caregivers globally given that social media platforms enabled her to connect with others all over the world. Essentially, social media apps were described by Sierra as a feasible means of creating virtual access to reach out to other caregivers. To expand on this experience of connecting with other 
caregivers through mobile apps, Sierra captured the following photograph to symbolize feeling connected to others:

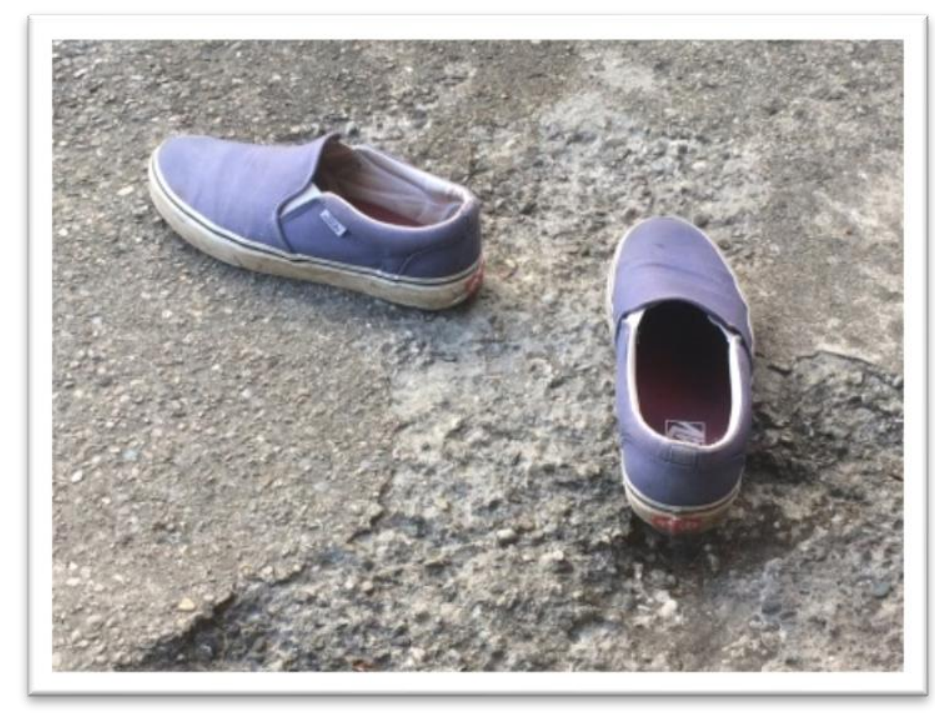

Photo 3. "Social media keeps you connected to other people; they don't have to walk in your shoes because they are already walking in ones that are similar to yours" - Taken by Sierra.

What I was kind of going for was like how social media keeps you connected to other people, other caregivers that you may not know or that you would have never met in real life and get you connected to those people. And I knew that I wanted to have something to symbolize the connectedness... they don't really have to walk in your shoes because they are already walking in ones that are similar to yours. And so, they can easily understand your experiences through their own experiences and you know, not everyone is going to be the same, but they can give suggestions as to what they did, and what worked for them and then you can kind of cater that for yourself. And so, that photo is kind of to show connecting with other people but that who knows what it is like.

Overall, participants used mobile apps to help with communicating and engaging with others as a way to foster social support from family, friends, and other individuals fulfilling the role of a family caregiver. Given that family members are described to be a significant source of social support, participants used mobile apps to enable them and their families to overcome practical barriers to communicating with each other. The photographs participants took conveyed the use of mobile apps in connecting with others and their social significance in how they enabled caregivers and the wider family to be involved in coordinating care. Accordingly, it is 
important to note that as a result of this sense of connectedness within the family due to effective communication is the ability for family members to plan appropriately when it comes to care provision for their loved one living with dementia. With the next subtheme, I discuss how participants used mobile apps to help with care activities in their caregiver role.

\section{Subtheme \#2: Assisting with Care Activities}

Participants described turning to mobile apps to assist with various care activities. Specifically, they used mobile apps to address two main areas of care provision: (1) activities of daily living [ADLs], defined as self-care tasks such as feeding and eating, resting, and toileting; and (2) instrumental activities of daily living [IADLs], defined as more complex skills needed to successfully live independently such as shopping, cooking, transportation, and managing medications and appointments with healthcare providers (Kim, Liu, Nakaoka, Jang \& Browne, 2018). Three key types of apps were used by participants to address those two areas of care provision including: reminder apps to help with both ADLs and IADLs; grocery- and fooddelivery apps to ensure grocery shopping and nutritional needs were met; and ride-sharing and car-renting apps to meet transportation needs.

Reminder Apps. Most participants expressed that using reminder apps or the reminder feature of apps helped them with both ADLs and IADLs for the care recipient, including managing medication administrations, making and keeping appointments with healthcare providers, and ensuring personal care needs (i.e. eating, resting, and toileting) were met.

Managing medication administrations was a caregiving activity that participants identified to be enhanced through the systematic use of reminder apps. For example, Cassandra described the use of reminder apps to help with managing medications for her grandmother: "So when she takes medication, she has little reminders on her iPad of what kind of medications she 
has to take and when." Likewise, Sierra also reported her family using a reminder app on their iPad for medication administration: "My dad has an iPad and he just leaves it in the house so usually we hear it go off and it has a medication reminder for my mom." Sierra further underscored the importance of reminders to family caregivers by stating "having reminders is a simple thing but it can help people so much, you know?" For Abigail, she began using the reminder feature of the Google Calendar ${ }^{\mathrm{TM}}$ app to ensure the safety of her father. As his memory worsened, he began to lose track of whether he took his medications or not. Thus, she decided to use reminders to ensure he got the correct dose of medication at the right time:

Because he would forget and then that's when I just start to [use reminder apps]. Because that would just make life easier because I wouldn't know if he actually ate it or not because he wasn't in front of me the whole time. So I told him that it would be better if I just hand it to him myself.

Participants also highlighted the importance of reminders to keep them on track as well in case they forgot. Thereby, Cassandra emphasized:

If she doesn't have her reminder app, I think I would forget too... I feel like that she is taking a lot of medications that it is kind of important to remind her and remind me so that she doesn't miss anything.

Managing appointments was also identified as an important function that could be strengthened by use of apps with the reminder feature. For instance, when asked how she organizes her grandfather's appointments, Sophia stated: “Oh yeah, Google Calendar!” Abigail also shared how she would use the Google Calendar ${ }^{\mathrm{TM}}$ app to keep track of her father's doctor appointments as it was more convenient than other methods:

I would say, I probably use apps up to three times a week and Google Calendar - two times a week because I just keep checking it and updating it. So let me give you an example, two days ago, a doctor called, he had an appointment scheduled for October $17^{\text {th }}$ and that's pretty far from now. And I will forget if I hadn't written it down so I usually put the doctor on speaker and say, "Hey can you just wait? Let me just put it on my calendar." Then it's already there so I know that no matter what, that's not getting touched. And I will put the appointments in immediately. 
Lastly, assisting with personal care needs and self-care tasks, such as taking a nap, eating and going to the bathroom, was another area of care provision that participants used reminder apps for. For instance, Cassandra used reminders for not only medications, but also to remind her grandmother when she should take a nap: "I would say that the ones we have been using, it works well for her, especially the reminder apps like when she should take her medicine or when she is suppose to take her nap. I think that works really well." Similarly, for Sierra, she also uses reminders for ADLs, such as eating and going to the washroom, for her mother who can no longer communicate her needs verbally due to the stage of her dementia. When sharing her photograph of a reminder notification on her phone, Sierra discussed in detail how she uses the reminder app in her caregiving role within the context of performing ADLs:

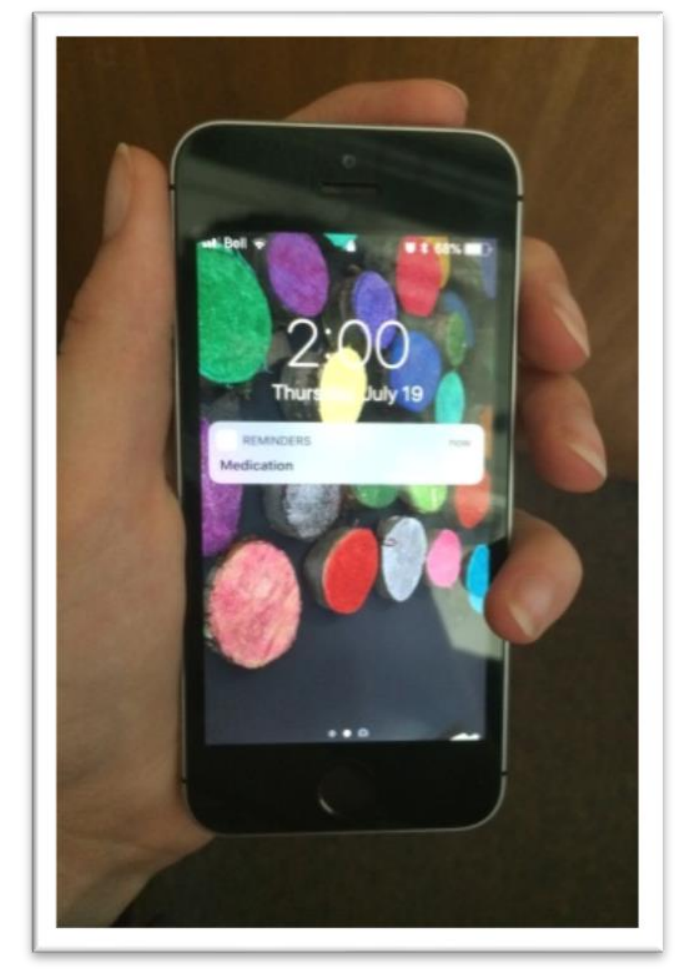

Photo 4. "Using a reminder to keep you in check of what has to be done, not just for you but for someone else" - Taken by Sierra.

So this, it's kind of to show how technology, how you can easily have reminders that help you remember things because I feel like it's always easier for me to remember taking my 
own medications than remembering giving my mom her medications. And so it's something that you may not think about sometimes - like having those reminders for another person is really helpful... Using a reminder to keep you in check of what has to be done, not just for you but for someone else because I think that it is harder to remember what someone else needs when they can't tell you. So another thing that I guess would help portray that even more - it's sometimes I don't realize "oh I need to take my mom to the bathroom" because she hasn't gone and it's noon or something and she hasn't gone since the morning but I would never think that she needs to go to the bathroom unless she told me and she can't really tell me anymore. Or same with like I don't realize that maybe she is hungry since she hasn't eaten since breakfast and it's almost lunchtime but it's hard for me to remember that she could be hungry because I am not hungry, and she can't tell me that.

Grocery-Delivery and Food-Ordering Apps. Nutritional needs were met through the use of grocery-delivery and food-ordering mobile apps by participants. Participants discussed the use of grocery-delivery apps, such as InstaCart ${ }^{\mathrm{TM}}$, and food-ordering apps, such as Uber Eats ${ }^{\mathrm{TM}}$, to order meals for the care recipient. Cooking is a challenge and a safety concern for their relative living with dementia and thus, participants often opted to order take-out meals when they are unable to cook for them or are not under the same roof. For example, Sophia reported using Uber Eats ${ }^{\mathrm{TM}}$ a couple of times for her grandfather: "I was going to say, I ordered Uber Eats a couple of times - I did sushi and Thai food." On the other hand, Abigail described using groceryand food-ordering apps on a more regular basis for her father:

I use the Instacart app every week! Apps that I use every week, on a regular basis, would be Instacart, Uber Eats, SkipTheDishes, and I recently started using something called DoorDash because it is just so easy to get things to my house if I am somewhere else and I want to make sure he is okay.

When presenting the following photograph, Abigail further elaborated on her use of the Kijiji $^{\mathrm{TM}}$ and TD Canada Bank ${ }^{\mathrm{TM}}$ app on her phone to order cultural food for her father that he particularly liked. By using the apps, it allowed her to tailor food to his specific personal preferences - even when she was away from home: 


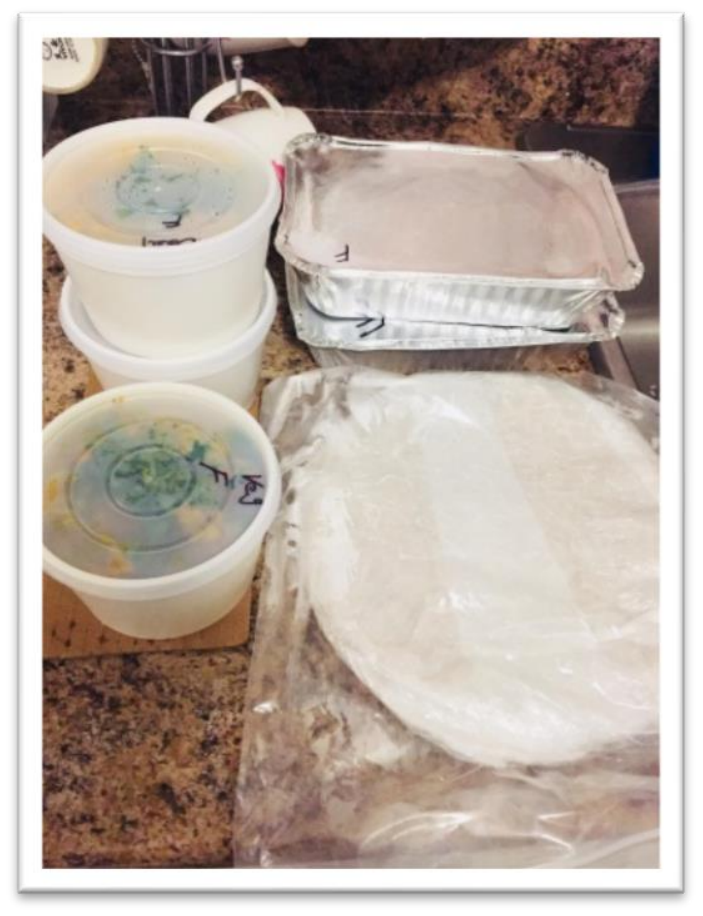

Photo 5. "I am sitting somewhere else and I can take care of him sitting really far away" - Taken by Abigail.

[Food] is probably the number one important thing in our family so I ordered it sitting from my friend's house... I was pretty happy, you know, I am sitting somewhere else and I can take care of him sitting really far away even... I used Kijiji because this is not even store-bought, well it is bought, but I don't know if you can tell, it's actually home cooked food... So I know my dad is very picky when it comes to outside food so I contacted five or six ladies and I kind of have their numbers on my phone but I found her through Kijiji. She doesn't live too far, she lives 15 minutes away and she's also from where I am so she told me that you know, whenever you need it, you call me and I e-transfer her, oh that's another mobile app I use!! So I e-transferred her 70 dollars and she'll deliver this food, so I guess I use TD and Kijiji for that.

Transportation Apps. Participants responded to transportation needs during care provision with the use of transportation-related apps. For example, Abigail's father can no longer drive due to his condition which made it difficult for him to travel to places. However, when Abigail wanted to celebrate her father's birthday by taking him to a restaurant, she opted to use a ride-sharing app called Uber ${ }^{\mathrm{TM}}$ to make it possible for both of them to do so: "I used Uber to get there and tried to get him a treat." On the other hand, Sophia used car-riding and car-renting apps 
to take her grandfather to medical appointments. Sophia recognized the challenges associated with taking her grandfather to medical appointments and sought out help from mobile apps such as Uber $^{\mathrm{TM}}$ and Car2Go ${ }^{\mathrm{TM}}$. She explained that Car2 $\mathrm{Go}^{\mathrm{TM}}$ would provide cars that were more comfortable and easier for her grandfather to get in and out of:

I would use Car2Go a lot. And because the car was a smart car, it was a smaller, shorter car. It was a little bit easier for them to get in and out of than a minivan which was what we had. We have a minivan and my grandfather had a hard time climbing up into the minivan. So it was easier for him to sit down into a smaller, shorter car. And so Car2Go was, it was a lifesaver for me too, to take them to appointments... I don't use Uber very much but maybe once or twice.

Additionally, this method saved her money which she valued as caregiving can lead to many expenses:

It would be cheaper to take a Car2Go because I didn't have to pay for parking. So I would get a Car2Go sometime to take them to their appointments... I got to find a way to save money... because I have to do it not just for my grandfather but my other two grandmothers. It adds up.

Most participants were keen on using various types of mobile apps to respond to the two main areas of care provision. In contrast, Nadia did not describe using mobile apps for caregiving in the areas of ADLs and IADLs as the current condition of her grandmother did not require her to do so. Nadia explained that not only is her grandmother relatively independent with her ADLs and IADLs, but her family members work with her at her business, so they are around on a daily basis to provide assistance if needed:

She's pretty independent. Basically, she goes to work every single day. She owns a building down on [street name]. And my family and her, they own a small business there... I just haven't been in that situation where we felt like we needed to use anything like that [mobile apps] especially since that a lot of our family members are always around.

However, Nadia stated that she would turn to mobile technology if her grandmother's condition worsened and if her and her family required more assistance with her care: "I'm definitely for 
technology. For sure. I think it's helpful. I think it is kind of where the world is going - that's the direction that it's going in. So I think I embrace it for sure."

To summarize, in the role of a family caregiver, participants sought out mobile apps to help with care activities, ranging from assisting with personal care needs to addressing transportation needs. While the use of mobile apps is prominent when participants take on the role of a caregiver, they also described using mobile apps when addressing their other roles and responsibilities beyond caregiving which is discussed further in the next subtheme. Participants noted that by keeping other aspects of their lives organized, such as personal, school and work, they felt more equipped to take on the caregiver role.

\section{Subtheme \#3: Organizing Everyday Living}

While all participants have the same amount of time in a day, how one spends and interprets this time differed and depended on numerous factors such as personal, school, and work demands. Participants recognized that time is finite and limited, and thus, required effective organization of all aspects of their everyday living. This was especially pertinent as in addition to managing school, work, and/or personal commitments, participants also had the role of a caregiver. Essentially, participants felt that balancing their multiple demands required effective organization and time management. As such, participants used mobile apps to not only organize the activities associated with being a caregiver, but also tasks pertaining to being in school, working full-time, and/or managing their personal lives. In this subtheme, participants discussed how they used mobile apps for areas beyond their caregiving responsibilities and how by doing so, they were able to be more prepared to take on the caregiver role.

Participants appreciated how mobile apps enabled them to complete tasks on the go due to its convenience and accessibility given that it can be accessed whenever and wherever on their 
mobile devices. By being able to complete such tasks in this manner helped them manage their multiple demands more efficiently. For example, Sierra expressed difficulty in managing the multiple aspects of her everyday living as well as her role as a caregiver:

Having a place, where little things that pop into your head, things that you need to do or things for life and everything that like you have to go buy this or little notes and stuff. I think feel like those easily get lost because there is always something you have to do like someone is always asking "do this" or like "can you grab this for mom" like "can you go get her clothes", "can you get her a sweater", "can you feed her". There is always something happening, you are always getting so much information from everyone else that sometimes your own things slip through.

Sierra further elaborated how mobile apps helped her organize the responsibilities associated with her everyday living as well as her role as a caregiver: "And also helping taking care of myself... that balance between what I need to do and what I need to do for my mom... And organizing my life as well." Sierra provided an example as to how mobile apps assisted her in organizing her daily life by describing how she could work on tasks using the Notes app on her phone when she is on the go:

Being able to save time as well so like when I am out, when I am taking the bus home, I don't always want to pull out my whole laptop if I think of something that I need to do or something. So just being able to write it on my phone and have it like sync to it a note on my computer as well so that when I get home and I pull out my computer, I see like oh yeah, I have to do this and get this ready or something. I guess it also goes with not losing your thought and being able to put it in and stuff when you're on the go.

In the same vein, Abigail also described the benefits of her using the commute time from work to home to complete tasks on the go via mobile apps: "I get a lot of things done on the TTC because that's 3 hours a day". Abigail further explained how mobile apps helped her manage the multiple demands she has by enabling her to complete tasks in any setting:

It made me feel useful too I guess in a way because it's like, you know, I have a really busy schedule so for me to be a caregiver at the same time, it's not easy. So the fact that I can be useful sitting at work, being outside constantly, I am a very, very busy person these days like I am gone from like $6 \mathrm{am}$ to $6 \mathrm{pm}$ and then I come home and sometimes I am gone again usually so the fact that I could do things made me feel definitely very useful. 
As a result, mobile apps helped participants save considerable amounts of time by allowing them to complete tasks in a timely and convenient method. For instance, Abigail stated that the time saved was especially valuable as this meant additional time can be allocated to providing care and spending quality time with her father. In particular, she indicated that despite the additional costs associated with ordering groceries via an app, the substantial time saved was worth it as it could be spent providing care and spending quality time with father and family:

It makes a big difference... And with the app, it would take me five minutes on the app, and I would have that hour and 30 minutes that I wasted on actually doing the grocery on taking care of him or spending time with him just because that is something that seniors usually like so in that way, it really helps. That app, like, is my life-saver. The InstaCart one... Yes, there is a fee. There is usually like a $\$ 3.99$ delivery fee and you pay service tax on top, and I usually always tip the person. So for me, total, it cost like per week, maximum, it's usually $\$ 15$. I think it's really worth it.

Abigail presented a photograph of a grocery store and explained in additional detail on how a grocery-delivery app freed up more of her time:

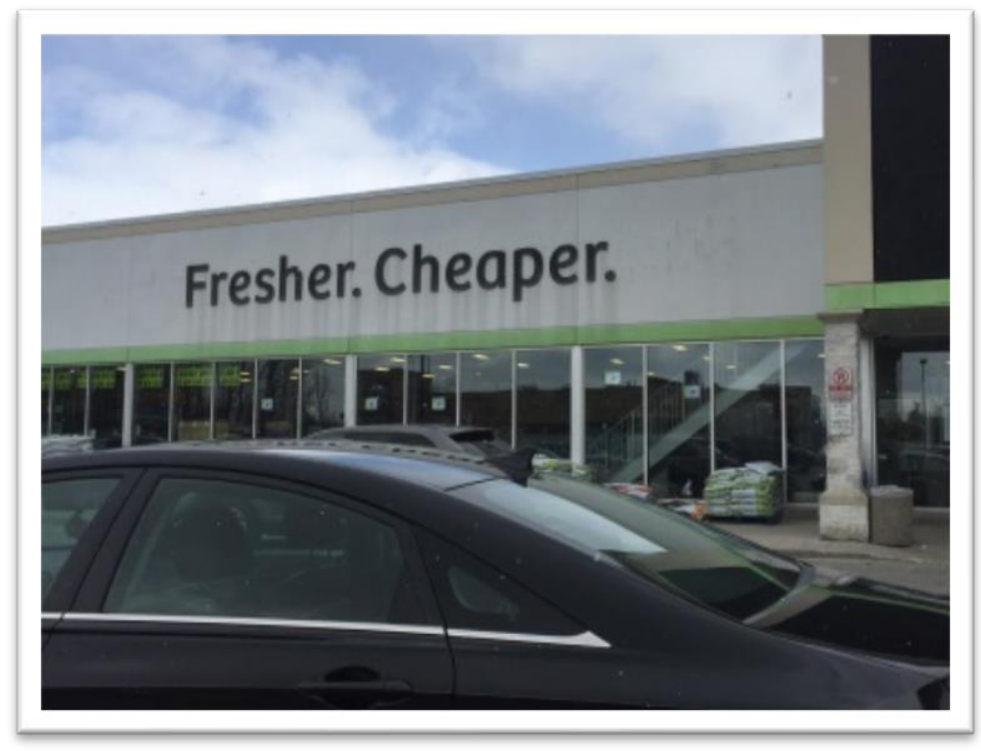

Photo 6. "I end up spending a lot more time necessary when I could just be at home and spend more time with my family" - Taken by Abigail.

I just kind of took that because I went on my way to work to FreshCo because I realized that I had to pick a couple things up, two or three. I said you know what, I am not going 
to pay $\$ 15$ to deliver two or three items. I went in there and I was instantly regretting it because I ended up wasting so much time and it reminded me kind of how important online delivery is for me because I became so used to getting groceries delivered that actually doing them was so hard. It may have been two or three items but I still spent 40 minutes in there so I was like you know what I am not doing this again... I always go for one thing and I end up spending a lot more time necessary when I could just be at home and probably spend more time with my family that way.

Participants also shared that organization-related apps, such as Google Calendar ${ }^{\mathrm{TM}}$ and reminders, not only helped with care provision for the care recipient, but also with organizing their other responsibilities. Accordingly, Abigail highlighted how she balances various tasks in her daily life through reminders: "The best way I try to balance things is reminders, I think I would be completely lost without them. I have reminders for everything." Further, Sophia also emphasized on the need to use mobile apps to stay organized with her daily activities: "I had to stay organized somehow and that was one way to do it: Google Calendar... Apps help me stay organized, stay connected." This was especially important given that they are often juggling the role of being a caregiver as well as other responsibilities, such as school, personal lives and work commitments. Not only do these apps help organize the care activities required for the care recipient, but also the responsibilities that the caregiver may have in their other roles. For Sophia, she presented a photograph of her Google Calendar ${ }^{\mathrm{TM}}$ app on her smartphone and her smartwatch to highlight the importance of having apps help her organize all of her daily activities, particularly those beyond caregiving: 


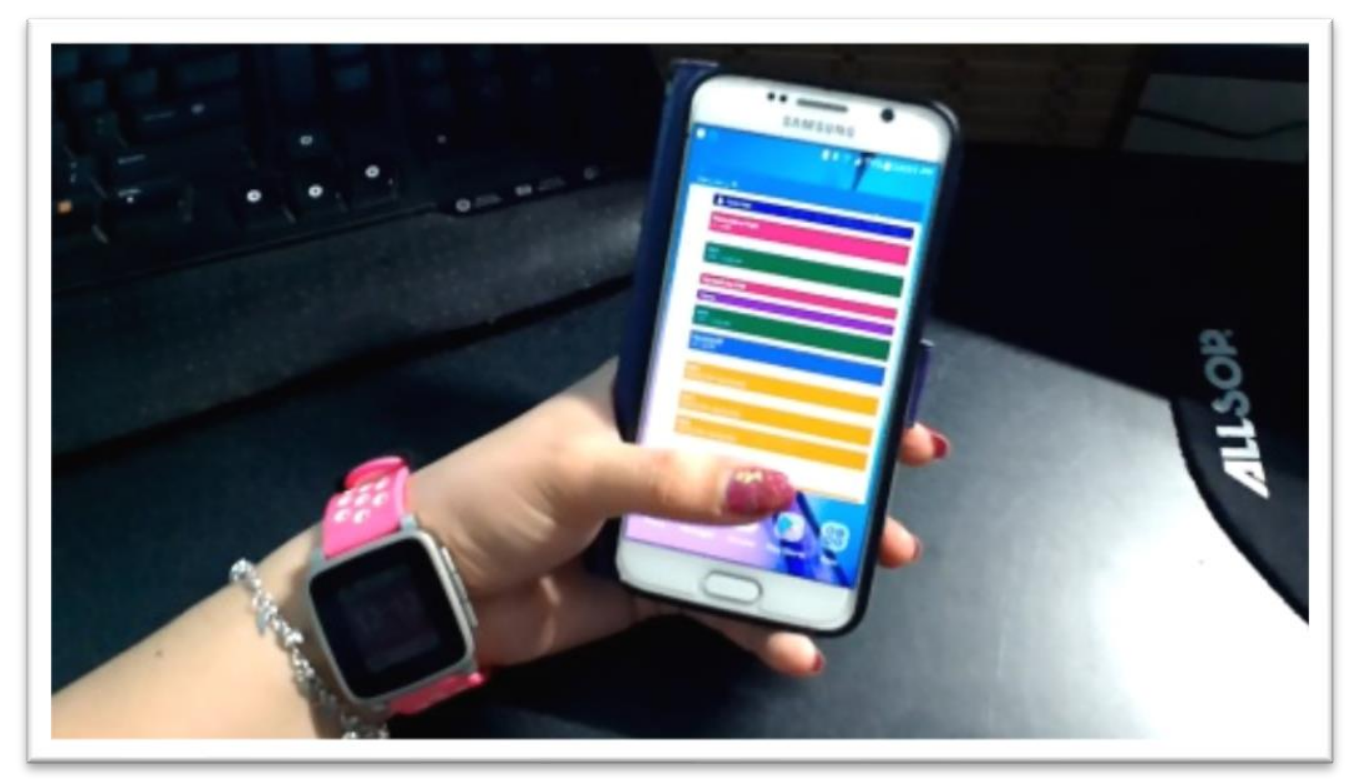

Photo 7. "That's how I kept organized - it was the only way I can mash up my personal life, my work life, and with having to care for grandparents" - Taken by Sophia.

On the topic of speaking of apps and technology and shows, I chose to show my calendar, my list of activities that I have listed and it never ends. Time never ends. There's always an endless amount of things to do and you can see the different colours. Each colour represents a different type of activity. And they're just all squished in one after the other. And they all represent something that I had to do. And that's how I kept organized - it was the only way I can mash up my personal life, my work life, with having to care for... I have more than one grandparent. I wasn't just caring for my grandfather. I was caring for my two grandmothers too. So with three grandparents and their schedule and their appointments plus my own personal appointments plus my other like things that come with life... Because like let's all be honest here like how would you have managed it without technology. Take technology out of the equation, I would be carrying an agenda around trying to and match some schedules onto a page of paper and if things change, I would have to remember to erase it and move it. And it would be a mess. And that piece of paper, that agenda is not going to beep at me and remind me.

Overall, most participants embraced having mobile apps in their life as a way to stay

organized in all the facets of their lives. Particularly, organization-related apps enabled them to efficiently manage other aspects of their lives beyond their caregiving role. In addition, various apps that participants used allowed them to complete tasks and activities in any setting, especially while they are on the go (e.g. during commute times). This in turn resulted in substantial time saved for the participants which they valued as managing the many roles they 
hold (i.e. caregiver, student, full-time employee) required considerable amounts of time. Although the use of apps in this context speaks to participants' need for organization in other aspects of their lives beyond caregiving, participants also recognized that the various roles they hold are inextricably intertwined. If they have one aspect of their lives well organized, it can influence their caregiving role. Ultimately, by effectively organizing the multiple demands that participants have, they can allocate more time and resources towards their caregiver role.

\section{Main Theme \#2: Minimizing the Impact of the Condition on the Person and the Family}

Participants described how dementia caused a disruption and change in the lives of their loved ones living with dementia as well as their family as a whole. Thus, in the face of their loved one's condition, participants spoke about the significance of upholding their loved one's sense of personhood by focusing on their identity, needs, and values. Additionally, they also felt that routines, traditions, and meaningful engagement were ways of maintaining stability and continuity - which was of fundamental value for the participants. By using mobile apps, participants found creative ways to address the inevitable disruptions and changes in their lives. Accordingly, this major theme consists of two subthemes. In the first subtheme, Promoting the care recipient's personhood, participants described ways in which successful uptake and use of mobile apps can support their relative living with dementia to maintain their sense of personhood, to cope more independently, and to increase individual autonomy. In the second subtheme, Maintaining family routines and traditions, participants discussed how mobile apps also helped maintain family routines and traditions by helping the family address the barriers that challenged upholding that routine or tradition. 


\section{Subtheme \#1: Promoting the Care Recipient's Personhood}

Participants spoke to seeing the person, not just the condition. Instead of persons living with dementia being defined by their condition, they are people and the condition is only one aspect of their lives (Kitwood, 1997). Participants recognized the need to celebrate who the person was, and who the person is now. Participants addressed the need to maintain personhood against a background of losses and restrictions that their loved one experiences, such as cognitive and physical challenges. For example, Nadia asserted the need to view persons living with dementia first and foremost as people:

Like of course it's important to be there as her caregiver but at the same time, I don't want her to lose the sense of her like her independence and her individuality... She's a mother. She is a grandmother. She's all these things. She owns a company. Yeah. There's lots to her for sure.

Similarly, Cassandra presented a photograph of her aunt to further illustrate this point and stated:

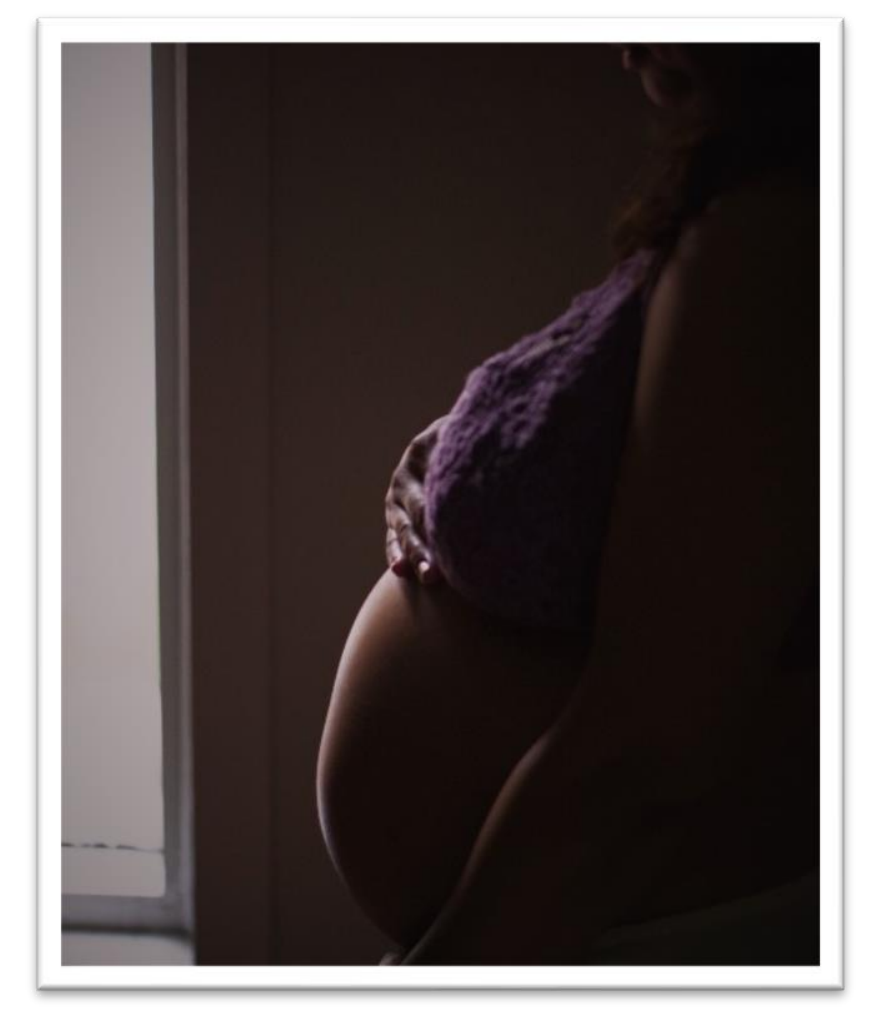

Photo 8. "A mother's love" - Taken by Cassandra. 
That is actually a picture of my aunt and I wanted to interpret in a way where that was my grandma long, long, long time ago. So it depicts a really strong woman who loves her children very much like she birthed three children and now she has so many grandkids, right? When I look at that picture, I just think of a mother's love and that's like how I would describe it. And so yeah, how I said, she... like back then, she really cared about her children and she was always taking care of them and it's kind of just like saying how now we are taking care of her too.

Participants believed that an important way for their loved ones living with dementia to preserve their sense of personhood was through maintaining meaningful relationships and activities. With an understanding of the importance of tailoring their care around their loved one's needs, preferences, values, beliefs, life history and other aspects that are important to them, participants were keen to turn to mobile apps to support their care. Participants were particularly interested in mobile apps that could sustain habits as well as support the care recipient to cope better, to act individually, to be independent and to engage in meaningful activities. Thus, participants commented that their relatives were still able to maintain previous hobbies, interests, and activities through creative means in the form of an app. For instance, Cassandra's grandmother uses colouring and puzzle apps to stay engaged and to help with her anxiety:

And for the colouring and puzzles, I think it kind of helps her calm down, kind of help her with I guess her anxiety kind of because she tends to get very irritated and I find that when she's using those apps, she is really calm and really quiet.

Cassandra's grandmother also used to enjoy gardening and can no longer do so due to her condition. However, the dyad was able to leverage a gardening app where she can continue to garden and plant - virtually: "There is also this one app where it is like a gardening app and she can plant, like she plants like virtual plants... And so, like she will plant them virtually and it makes her really happy." The use of an app not only helped Cassandra's grandmother manage her anxiety, but it also gave her a strong sense of mastery and self-efficacy which the participant felt was beneficial when promoting her independence and sense of personhood: 
I think the most helpful one would be the puzzles but obviously, not too complicated puzzles. Say like a picture of a flower. Yeah so, I think that would like really help.

Because it kind of, you know how the puzzles are not together yet but she kind of put the pieces together. It kind of gives her a really, I don't really know that word; it's like a feeling of... [accomplishment]. It's like when she sees the final picture, it's like "oh my gosh, yeah, I did that!" And it kind of helps the brain I guess.

Moreover, for Nadia, she illustrated the passion that her grandmother has for music

through a picture of a guitar and vividly described an event that happened when her grandmother was recently hospitalized to which music played a huge role:

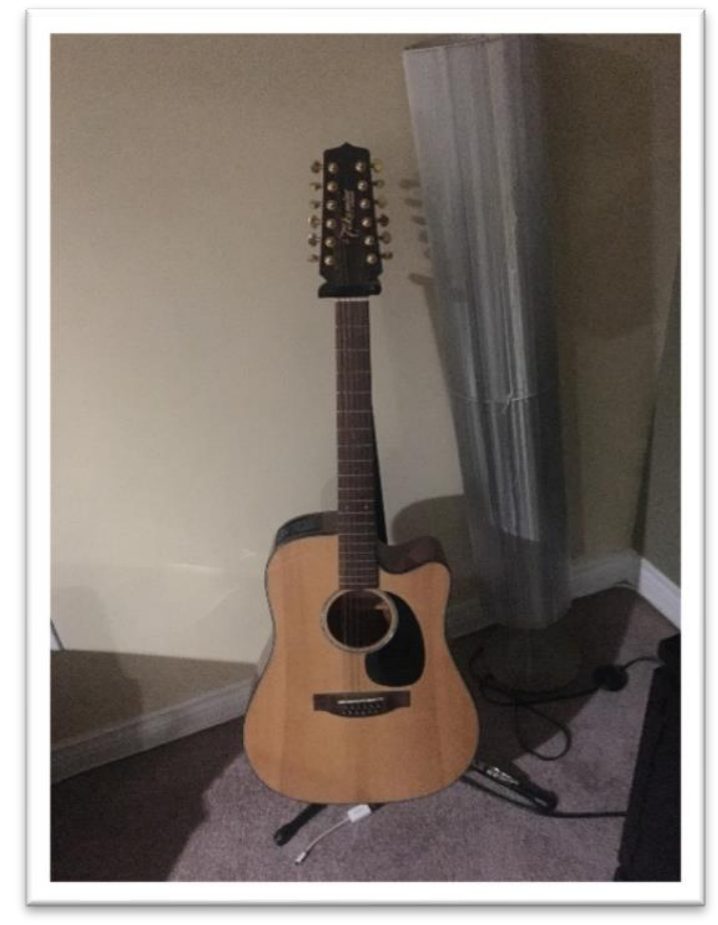

Photo 9. "For her, everything goes around music" - Taken by Nadia.

So the guitar, so music as I said, actually for her, everything goes around music pretty much... When she was in ICU, my one uncle... he came to the hospital and he did kind of like a song for her and my other uncle participated in it too. And I honestly don't know if it was just luck of the draw or whatever but having people in there singing, that's when she started improving. She was ventilated and they thought that she was definitely not going to make it through. And she was out the next day to the cardiac unit so I don't know what it was but I think something about it kind of gives her hope: the music.

Thus, recognizing the key role that music plays in her grandmother's life, Nadia went on to explain that she would use the YouTube ${ }^{\mathrm{TM}}$ app to play songs that her grandmother would enjoy 
whenever she would visit her throughout the last five years. By engaging and connecting through music via the YouTube ${ }^{\mathrm{TM}}$ app, Nadia and her grandmother were able to further build a stronger bond with each other:

I think it really brings her back to a place where she is focused and interested and happy. I think it brings her a lot of enjoyment to look at pictures and listen to music... I think it definitely, definitely brings up her mood, makes her happier. In terms of how it affects our relationship, I think it's really, really nice to get to bond through those little acts because she's just so happy when she's using them.

From their narratives, it was evident that participants understood the importance of not only caring for the physical aspects of their relative, but also to cultivate their sense of personhood and enjoyment. Even when severe cognitive and physical decline was apparent, participants tried to respond to their relative's needs and preferences using mobile apps so they can continue to engage in activities which were still meaningful to them. For instance, Sierra described that while the stage of her mother's condition limited activities that she could participate in, she would still turn to her mobile devices, such as her smartphone or laptop, to watch videos or view photos with her mother in an effort to engage her in meaningful activity: "Sometimes, I would just pull it [phone or laptop] out to show her the video or a photo, or pull my computer and watch a show on it."

Similarly, in the face of her grandfather's significance decline, Sophia discussed that the need to maintain a sense of enjoyment and engagement in her grandfather became increasingly important:

He is still living in his own way. He is just not physically able to and it doesn't mean that he can't live... There are other ways to care for somebody and it isn't always making sure that they have eaten for the day and that they are washed and ready for bed and comfortable. There are the mental aspects that you have to take care of too - even though they're not mentally there. 
As exemplified in the following statements, when her grandfather was hospitalized, Sophia described in detail how she used the YouTube ${ }^{\mathrm{TM}}$ and Netflix ${ }^{\mathrm{TM}}$ app on her tablet to play videos that he enjoyed. Made possible by mobile apps, she was able to cater to his preferences and provide entertainment in a personalized way. Sophia presented two photographs to signify the importance of promoting her grandfather's sense of personhood by tailoring her care around his preferences and by providing meaningful activities for him during his hospitalization:

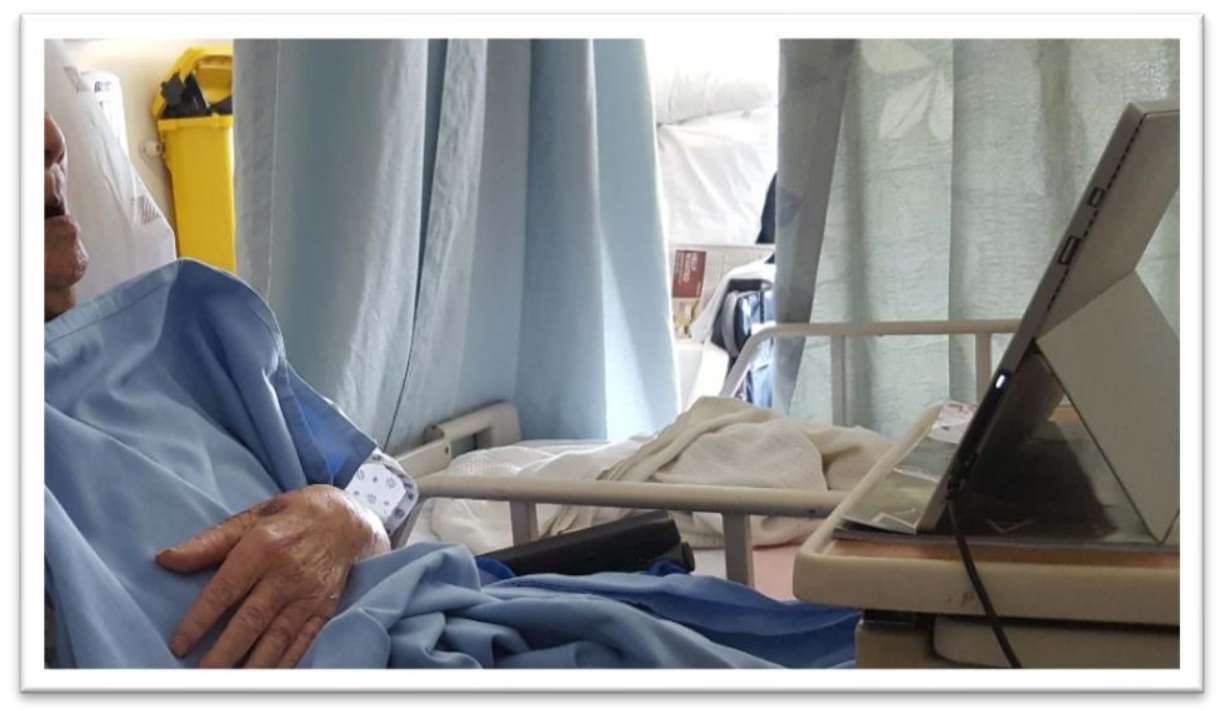

Photo 10. "You can still bring some new experiences for him, for the person, just with technology" - Taken by Sophia.

That's my laptop tablet. And I was playing a video for him... It's a picture of him watching YouTube. And he's watching that World War documentary that I told you about. He loves watching War documentaries and watching about the Nazis and the Japan war stuff... You can still bring some new experiences for him, for the person, just with technology... I wanted to show that this was possible because I had a) the Internet; b) a portable device that I can show him these things on. And see that the information was actually out there to bring to him to see that... He still couldn't walk, he couldn't run around and it's not like he can climb Mount Everest. But at that time, the least I could have done was give him something that he personally enjoyed and that he personally loved... It was very personalized. 


\section{NETFLIX}

Deady to Cast

目

Photo 11. "I was using mobile technology like Netflix... We wanted him to stay engaged" - Taken by Sophia.

I wanted to show that I was using mobile technology like Netflix. I was able to put it up on a screen to show different videos or shows like make it visible for him... We wanted him to stay engaged. We didn't want his last moments of life to be slumped over in a bed and staring at four walls until his mind went completely nuts. I think for any living person whether or not it was 100 percent alert and oriented versus confused and forgetful, for any person who is still able to hear, see, talk, feel, touch, you shouldn't have to sit there and do nothing. Do you understand? Like if you still can live, you should live in whatever way you can live. If it's whether through other people's experiences which is through a screen or pictures maybe you can't physically do it yourself but you can still live it.

It is through the involvement in familiar and meaningful activities that the family

member living with dementia is able to gain pleasure and inclusion as well as maintain a sense of continuity and identity, which are critical for establishing personhood (Kitwood, 1997). As such, participants played a key role in promoting their loved one's involvement in meaningful activities through the use of mobile apps as a way to sustain their personhood and well-being. In this subtheme, the significance of meaningful activity is discussed on an individual level, but the study data lent support to the notion that activity can also matter deeply at a family level which is further explored in the next subtheme. 


\section{Subtheme \#2: Maintaining Family Routines and Traditions}

While familiar and meaningful activity for the family member living with dementia is important on an individual level, participants noted that activities in the form of family routines and traditions were also valuable to the family as a whole. When a member of the family is living with dementia, family routines and traditions can be disrupted as well. By continuing to engage in long-established, shared family routines and traditions, it can promote a sense of stability and continuity in the lives of the participants and their families. Knowing this, participants described their ability to be resourceful and seek out mobile apps that can help them overcome a specific barrier when maintaining family routines and traditions. It was in this context that some photographs captured by participants became particularly poignant.

One salient example was when after teaching him how to use mobile apps, Abigail's father was able to order a birthday cake for her to celebrate her birthday through the use of an app called Uber Eats ${ }^{\mathrm{TM}}$. When sharing a photograph of the birthday cake, she described how without the app, it would have been challenging, or even impossible, for her father to get her a cake for her birthday since he no longer can drive due to his condition. This family was able to uphold the tradition of celebrating birthdays with cake as the use of the mobile app empowered the father to give his daughter a special gift for her birthday. This event further demonstrates that not only are the caregivers using mobile apps to express love and appreciation towards their loved one, but also vice versa. 


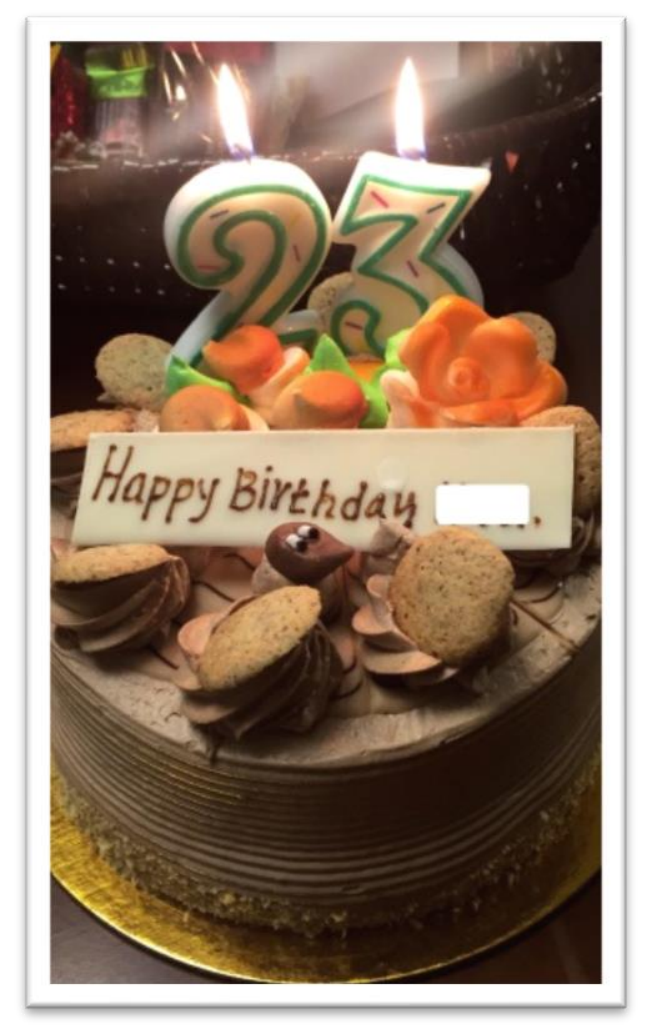

Photo 12. "He wanted to get me something for my birthday; he ordered it on the app himself; and I was proud of him" - Taken by Abigail.

He kind of really wanted to get me something for my birthday but he couldn't because right now, because of his Alzheimer's, his license has been temporarily revoked ... So what he did was, which was so sweet, he went on Uber Eats and he ordered a cake from there... I was proud of him. He actually did that for me. He was like you know what? I can't do anything myself safely. So that's why I took that picture. I was just like shocked that he actually went and did that... I use it for food but I realized that you can even order cakes. You can practically order anything now.

Abigail also went on to describe how she used another food-ordering app called

SkipTheDishes ${ }^{\mathrm{TM}}$ to reduce the burden of preparing for Eid celebrations, a family cultural tradition that occurs every year. She ordered catering from the app for up to 45 guests, so they can still celebrate with friends and family in a big gathering despite the challenges of caregiving and her father's condition. By using the app, Abigail articulated that it would result in "spending time as a family and enjoy, rather than slaving two days before the actual event". As illustrated by Abigail in the following photograph and ensuing dialogue, the use of mobile apps opened up 
possibilities to maintain family celebrations in a way that would not have been possible and too burdensome for the family:

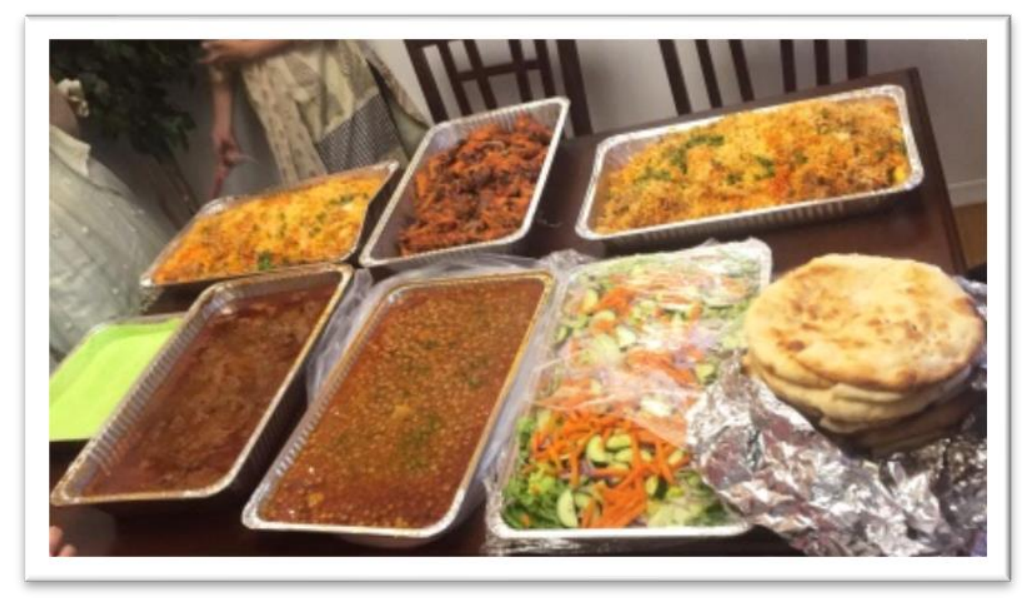

Photo 13. "We can spend time as a family and enjoy; rather than slaving two days before the actual event" - Taken by Abigail.

We are Muslim so Eid passed this week so we had a party of I think 45 guests and I got the catering done from SkipTheDishes. So all of this is food related so I realized it may be a little bit repetitive. But I used a different app... I used a different app this time so even I was surprised that I can get catering done so through an app. Before, I used to think that I would have to place an order weeks ahead or something. I ordered it two days before and I actually called them to make sure because I was like I don't know, do they really do catering through an app? But they do!

For Sierra's family, they have a routine of taking photos and videos of everyday life and of significant events using the camera app on their smartphones. She went on to describe the importance of documenting her mother through pictures and videos in particular. By doing so, it gave her the opportunity to capture various moments in her life to reflect and reminisce on:

I'd say probably the camera and the photos app I look at and I document times with my mom... Like to use the camera app on my phone to take pictures and especially taking videos of like my mom. It is really helpful for me because it's something for me to look back on. I get to see what happened before and see the progression of everything.

Sierra further expressed how apps helped her family uphold this family routine by allowing them to share photos and videos with each other conveniently: "A lot of times my sister would be like pull out her phone and take a picture and I would like oh send that to me!" 
Similarly, Nadia and her family also have a longstanding tradition to consistently document family events and other memories through pictures and videos. This was particularly important for the participant's grandmother as this tradition has been an integral part of their family life for many years. Capturing pictures and videos, now through a mobile app, remained an important activity that allowed the family to be involved in a familiar routine, thus providing a sense of continuity. Nadia shared a photograph of her previous digital camera to exemplify the significance of upholding this tradition within her family:

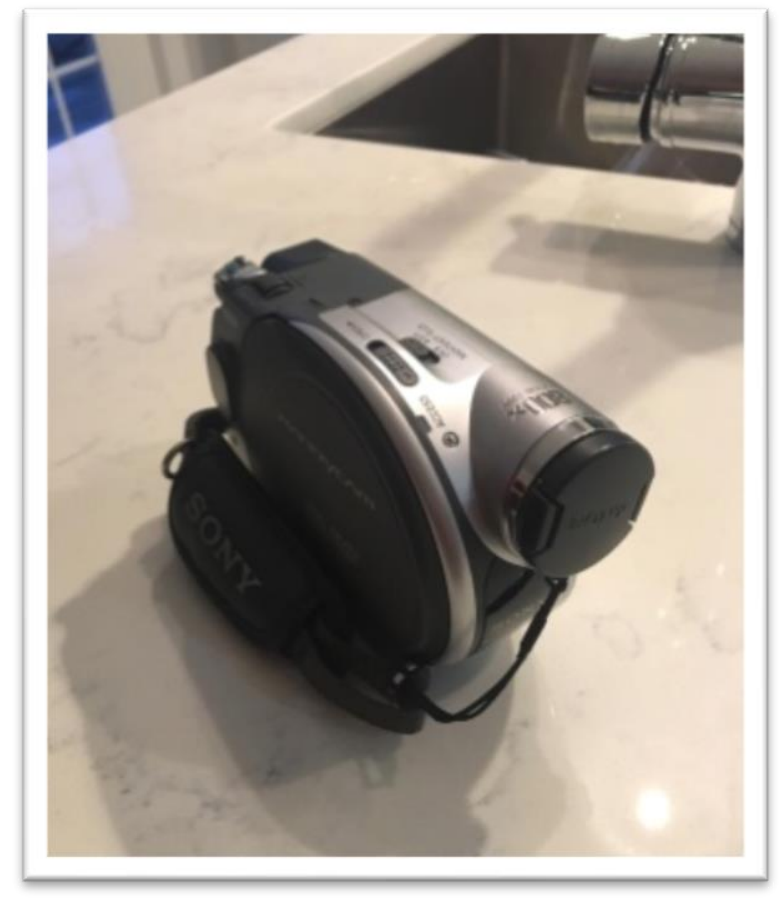

Photo 14. "She loves to have that kind of togetherness and the memories" - Taken by Nadia.

So the video camera, you don't see those too, too often. We don't necessarily use that as much but what I do remember is that my dad and my grandpa so my grandma's husband, he's passed away, but when he was alive, he used to take videos of everything with his video camera. My dad and him as well. So that's what kind of reminded me of it because my grandma always would like "oh take a video of this, take a video of that. We have to have this." So she loves to have that kind of togetherness and the memories. So again, the videos that we showed her from when we were children or when her kids were young. And she gets so happy and sometimes she can't believe it. She has old pictures hanging up in her front foyer of her house. So she really likes that. She has a stack of old photos just hidden away in boxes in the back of her house. So for her I think like video cameras 
and cameras and memories like that, that's what is most important to her... Now it's switched to our phones for the video camera... it's definitely easier to use a phone now.

Continuity within the family context through involvement in shared family activities was described to be deeply important to participants. Although the condition of the care recipient may result in many changes to their lives, participants were able to navigate those changes by accessing mobile apps to help maintain family routines and traditions. By using the mobile apps, they built a connection to the usual family life they had led with their family members, and as such, created a sense of continuity with the past.

\section{Main Theme \#3: Determining the Effectiveness of Mobile Apps}

A central issue that was the biggest driver for uptake of mobile apps for all participants was whether or not the app was effective in helping them address an unmet need. Accounts from participants suggest that during their process of determining the effectiveness of mobile apps, they explored what works, what doesn't and what's to come - and the following two subthemes reflect the results of that exploration. In the first subtheme, Identifying useful functions of mobile apps, participants described characteristics and functions of mobile apps they found useful and how they promoted continued use. On the other hand, in the second subtheme, Envisioning the potential of mobile apps, participants illustrated areas that mobile apps could improve on as well as the potential of mobile apps in addressing some of their challenging caregiving needs.

\section{Subtheme \#1: Identifying Useful Functions of Mobile Apps}

Participants discussed reasons why they would use a certain mobile app in their caregiving role. In doing so, they outlined several key characteristics and functions that mobile apps must possess in order to be perceived as useful and to be continually used in their caregiving role. The main areas of mobile apps participants identified as useful to them as 
caregivers are grouped into three general categories: Simplicity, Easy Navigation, and Interactivity and Integration.

Simplicity. All participants valued simple, practical and easy to use functionalities in mobile apps. Specifically, they appreciated when an app has a simple interface (how the mobile app looks) where it is more intuitive to understand how to use the app. In their descriptions, a simple interface included aesthetics that are appropriately styled and attractive yet not overwhelming as well as a format that is simply laid out. For an app to be perceived as "simple", it must include a minimalistic design where there is reduced clutter, the content is kept to a minimum of what is relevant by presenting the user with only what they need, and the use of simple language is supplemented with visuals. The app should not be text-heavy and should have minimal jargon and technical terms. For instance, Sierra illustrated that simplicity and minimalistic design are qualities she valued in the mobile apps she uses regularly: "More simple and also like minimalistic-like interface as well. I only see what I need to see." In accordance to Sierra, Nadia also stated the main reasons she uses the mobile apps that she does is because of their easy to use and low effort functionalities and as such, she would recommend those apps to others to use: "It's definitely really easy to use. Yeah so that's a plus for sure. I would recommend it to others - Definitely! I think it's a go to app for a lot of people. Right? YouTube, FaceTime - easy, simple and quick."

Easy Navigation. Participant feedback also highlighted the importance of navigating through apps easily, such that they are obtaining maximum useful outcomes from an app with the minimal amount of input necessary. Participants stated that finding key features and the navigation of the various aspects of the app needed to be easy and straightforward. For participants, this is often achieved by offering clarity in instructions, meeting the user's goal with 
as few steps as possible, having navigation of the app be logical, inituitive and self-explanatory, as well as presenting content in a natural and consistent manner that suit the purpose of the app. For example, Abigail illustrated the ease behind navigating her reminder app: “The reminder app, obviously, that's there and the accessibility, just about opening it very easily." Similarly, both Nadia and Sophia discussed how easy it is to navigate and achieve their goals through the apps they use. They alluded to the inituitive manner of their apps in which navigation is easy and straightforward. Nadia stated, "YouTube is just easy because you can basically search anything, and you'll get a result and then FaceTime also quite simple because it is just like an outgoing phone call basically except with the visual piece." Likewise, Sophia spoke of similar sentiments about YouTube ${ }^{\mathrm{TM}}$ and Netflix ${ }^{\mathrm{TM}}$ : "It's easy, straightforward...And the amount of content that we can access. The variety."

Furthermore, Cassandra highlighted the importance of these functions when she described a colouring app that her grandmother uses and explained why this app was perceived as more useful than other similar apps they tried. Cassandra emphasized the need for apps to be simple and straightforward with minimal text in order for her grandmother to use:

For the colouring one, when you open the app, it doesn't give you any instructions. It's just the paint brush is there and the colours are there and she can just click on the colour and put it on the picture so it's not really "oh you have to do this and then at this time". Because the [App name] one, it was like timed and it was giving a lot of instructions as to how you should do it and when you should do it. So with the colouring one, you could just do, it's really easy.

It is evident that for participants, if an app was perceived as too complex, the possibility of mastery of the app reduces and as such, successful uptake and use diminishes rapidly. This was especially pertinent when the app was intended for the care recipient to use.

Interactivity and Integration. Certain mobile apps allow the option for interactivity whereby users can enable the sharing of information to others. For Abigail, this feature of a 
calendar app she uses was a function she found useful as a caregiver given that it allowed her to share her calendar with her family members in order to update them about her father's activities and to coordinate care. Abigail further elaborated that this opportunity to share information was especially valuable when the family members reside in far away area:

A feature that I do like to use is the fact that I can share things and invite people through the Calendar. There are times where I might actually have to show my sister something, like I share the event, the calendar, everything with her even though she doesn't live here. So that's really amazing that she doesn't have to live here; she can live far away and still see everything. I like the sharing aspect.

Given that mobile apps have the ability to be integrated and available on different mobile devices, including laptops, phones, and tablets, users could easily access the app through any device. For Sierra, she stressed that this capability of apps where one could access the same app and features through various devices was important due to the convenience and accessibility that it provides. As a result, she can choose to complete tasks on mobile apps through whichever device that is suitable at the time (e.g. smartphone on the bus versus laptop when at home):

Like not losing anything, continue working on it... So I think a lot of it is not forgetting things and having multiple ways to remind me and being able to like to sync calendars and stuff like that between my devices is really important and then I think it saves time as well because you can work on something on the go on your phone and then later, pick it up on your laptop when you're like at home or stationed somewhere.

To expand on her experiences, Sierra captured the following picture to symbolize the impact of this mobile app function on her as a family caregiver considering the "busy and chaotic" nature of caregiving: 


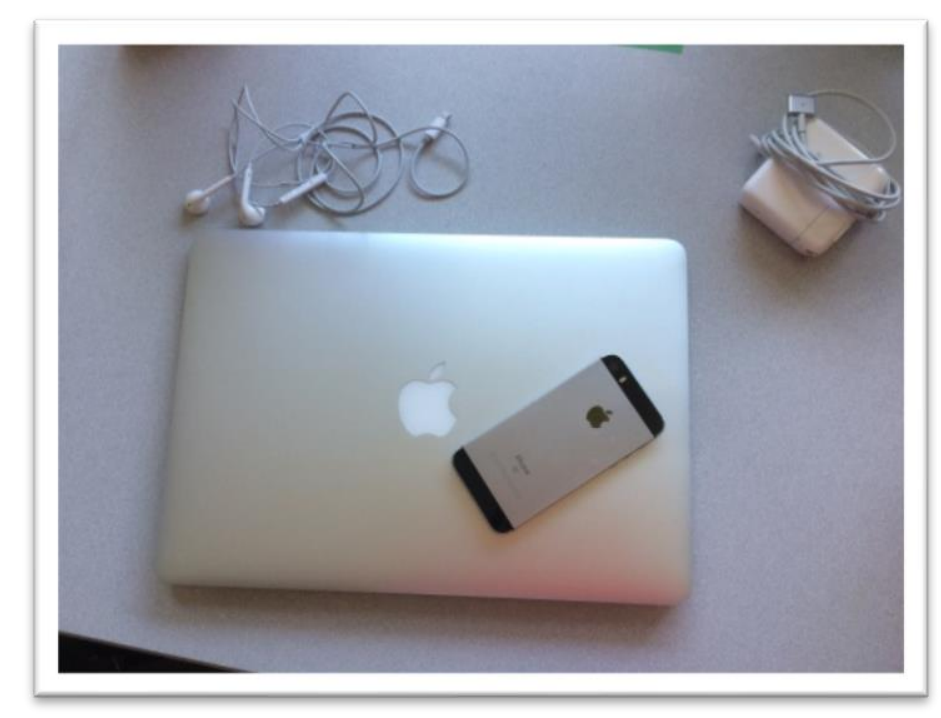

Photo 15. "Integration is really important especially in caregiving so you can stay connected to what you were doing no matter what device you are using" - Taken by Sierra

This one was more about the integration and how for me, that's really important especially like caregiving - if I can start a task on my phone when I am like on the bus home and then finish it on my computer once I actually get home because like saving myself time is really important. So if I think of something that I need to put in my calendar, it's nice to be like able to put it in my phone when I am out and then have it sync to like my computer right away. And so that photo was a lot about integration and not just between my like Apple products but also like having mobile apps that you could like also access through like websites as well so you can like stay connected to what you were doing no matter what device that you are using... I think that's really important because everything is so busy and chaotic like as it is and as soon as I get home, there is always something I need to do right then for my mom or for my family.

Participants revealed that simple, practical apps with immediate benefits, are most likely to be implemented by them in their caregiving role. They also felt that clear, intuitive navigation was important, with the ability to easily find data and achieve desired outcomes within the app. In terms of simplicity and easy navigation, participants emphasized that these two characteristics are required in order for their loved ones to use the mobile app. If the app is too complex, then the care recipient is unable to use it independently, leading to discontinued use. Lastly, participants discussed the importance of interactivity and integration as these features allowed them to share relevant information with other family members as well as access to the same app 
on various mobile devices. For them as family caregivers, these two functions of mobile apps provided two main benefits: (1) ability to provide updates and coordinate care with family members in a convenient manner; and (2) ability to complete tasks on an app using whichever mobile device suitable for the context and setting. While participants discussed the useful functions of apps that promoted continued use, they also described areas in which apps can improve on and the potential of apps in addressing some of their caregiving needs. This is further explored in the next subtheme.

\section{Subtheme \#2: Envisioning the Potential of Mobile Apps}

Participants discussed a wide range of possibilities for mobile apps in the context of addressing the many, yet diverse, challenges they face regularly as caregivers. Given that each participant has different caregiving experiences, the ideas they provided varied according to their perceived needs. Participants mainly focused on two areas, the functionality of the app and the purpose of the app, when describing what type of apps would be beneficial for family caregivers.

In regard to the functionality of the app, Abigail and Sophia both provided some insight. Abigail suggested developing apps that could act as a "one-stop-shop" where an app could have multiple functions for increased convenience and to decrease the clutter when one has too many apps with one singular role or purpose:

The one thing I would do better is kind of integrate a bunch of things to one app. For example, I have three different apps doing three different things. So like an app where I can not be too stressful, maybe like do groceries and then book appointments and speak with doctors, like something with an integrated network. Or something like that. That would be helpful.

This way, an app can address the many and complex caregiving needs of family caregivers within a single app, rather than relying on a number of apps with singular features and functions. Sophia commented that language barrier prevented the use of mobile apps for her grandfather and therefore, she suggested the development of apps with the option of having other languages: 
"You can say language barrier because my grandparents can't use it." Sophia highlighted the need for mobile apps to cater to individuals with varying culturally backgrounds who speak different languages.

Upon reflection of the caregiving needs that they face, many participants described how mobile apps could be designed with the purpose of addressing one of those needs. For Sierra, she offered three ideas she hopes that can be materialized into an app. These ideas cultivated as a result of the many challenges she faced as a family caregiver to her mother who is at a later stage of dementia. First, since Sierra's mother receives homecare, she proposed the design of an app that would allow family caregivers to communicate with healthcare providers more efficiently. She explained that this would facilitate the involvement of the family into the coordination of care activities which would ultimately, promote more family-centered care:

I think the biggest thing might be especially because we have all these PSWs that come in, especially when there are people that haven't been here before. Like someone went on vacation, like a regular person that has been here before, and a new person is coming in. I wish there was a platform where we could put in the tasks they would be doing or where they could find things in our house so you don't have to kind of hovering around and making sure they are doing things right or in the right order in the same routine as the usual people. And having that as a space where they can check in and also add in things and then we can add in certain stuff and that could be helpful for them as well. I think what's missing is that communication between this is how we do it in our family and this is how they do it with their other clients.

Second, another suggestion provided by Sierra is an app that organized the care recipient's activities of daily living. In this case, similar to what Abigail suggested, Sierra wanted an app that is multifunctional where it can possess many functions related to managing aspects of everyday activities, such as tracking food and fluid intake and monitoring toileting. Given that her mother is in the later stages of dementia where she is no longer able to communicate her needs verbally, Sierra asserted the benefits of having a mobile app assist her in managing her mother's activities of daily living: 
So even putting in a reminder like oh a 10:30am snack reminder and then that would really help to remind me of what I have to do for her when she can't. Like that might be something that she needs but she can't tell me. Having structure and routine is also important too. And that can be easily made through reminders and stuff like that too. You would have to put in a lot of like information but say, you know the rough estimate of how much liquids they're drinking in a day or how much they should drink in a day or how many meals they would like to eat in a day, and you put that information in and so it all reminds you if you haven't input it recently. Oh like they need to drink more water or they're drinking a lot of water today, maybe they should go to the bathroom and things like that - it's like the app can kind of keep track of information and put reminders out, maybe not on set schedule all the time, but as needed.

Lastly, Sierra described that her mother would go to a day program on weekdays with the help of a transportation company that caters to individuals that travel in a wheelchair.

Unfortunately, because of the uncertainty of when her mother would arrive after she is done at the day program, this often meant her and her family had to make sacrifices in order to wait at the house for her mother's arrival on a regular basis. She stated that she would value an app where she can track the times of the bus and when it will arrive at home, similar to an app available commercially by the Toronto Transit Commission (TTC):

We have that [the TTC app] in [city] and it was when I was taking the photo, it is such a simple thing but it is like we don't have that and it would be so easy to create and yet, without it, it causes a lot of problems and increased stress and everything.

Sierra took the following photograph to symbolize this experience and to further emphasize the potential of mobile apps to address this particular challenge which causes a lot of stress for her and her family: 


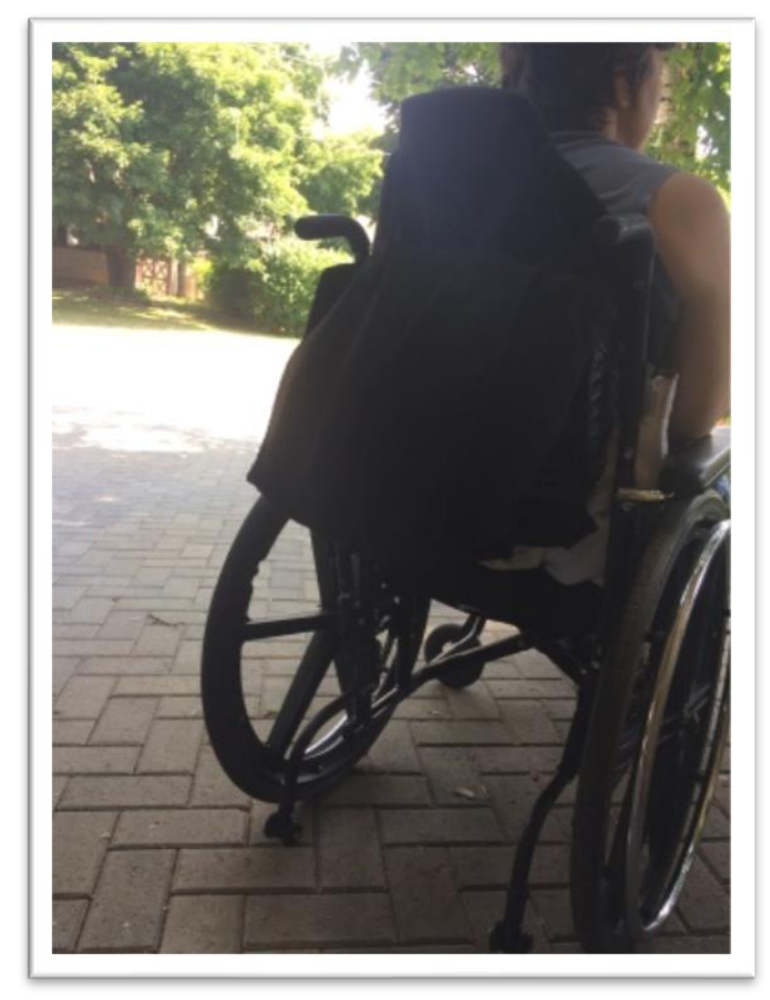

Photo 16. "I would wish there could be an app to know when she is getting off the bus so that we're sure she is there, and the bus didn't make our lives harder" - Taken by Sierra

So this one is kind of about what like I wish there was... when my mom gets off the bus, we don't really know exactly what time she is coming and that can cause a lot of problems with my family because it is hard to plan around that. Like make sure my dad is going to get home from work or if my sister has to get home earlier or things like that. Especially when we have a family dinner on Fridays at my grandparents' house so we have to eat quickly and rush back even though she might not be there until an hour later. So sometimes we are rushing when there is no need to be so this photo was kind of like to represent like her sitting alone because like I guess they would just leave her... Because I sometimes feel like she is alone and she can't, if we are not there, then what is she going to do? Like I said, with the technology piece tying in, it was what I would wish there could be an app to know when she is getting off the bus so that we're sure she is there and the bus didn't make our lives harder... Or we are like constantly waiting for her looking outside and they're not like coming by... Usually like picking her up to go in the morning, they're always like on time. Sometimes, they're a little bit early but it doesn't matter as much but they're never late. But coming home, she could be dropped off like anywhere from like five to seven.

This idea by Sierra underscored the notion that family caregivers are not seeking cutting-edge apps but instead, already available solutions might be just as important in addressing their caregiving challenges. 
Commonalities were found among a couple of participants where they stated safetyrelated concerns as an area of caregiving in which they hope mobile apps can support. Specifically, Nadia described concerns regarding forgetting to turn off appliances and falls at home which she hoped mobile apps could provide assistance with:

I think the thing that I would be most worried about would be her leaving the oven or the stove on. Yeah, that's what concerns me. Or if there is a fall, or if she falls or something which doesn't happen often but yeah. And then no one being there to help her out. I think those would be what I would be most concerned about.

Sophia had similar concerns and explained that she would like a mobile app that could provide support during incidences where her grandparents had a fall or another form of emergency: "Fall or emergencies because there were two incidences where they had an emergency and they didn't know how to call the police". She further elaborated that a mobile app that can help her track her grandfather when he is wayfinding would be beneficial: "He's a wanderer as a dementia patient. And I know that there's something like that already starting to come out. You can track them. It would be nice to be able to track him.”

In conclusion, participants discussed their vision of how mobile apps could potentially address some of their current caregiving needs. They provided some suggestions to improve the functions of apps to make them more effective and useful, such as having multiple language options and being multifunctional. They also offered some ideas of specific caregiving needs an app could address, including communication with healthcare providers and management of safety-related issues (e.g. falls and emergencies). 


\section{PART II: PARTICIPANTS' EXPERIENCES WITH PHOTO-ELICITATION INTERVIEWING (PEI)}

In this section, the findings address one of the research sub-questions which pertained to the study methods of using PEI as a data collection method: What are family caregivers' experiences of using photographs as a conduit for explicating their user experiences?

Particularly, this section focuses on the participants' experiences of engaging in PEI. All participants responded to the photography activity positively, produced multiple thoughtful photographs, and discussed them actively. All participants took five photographs, with two participants choosing to submit more than five. In all, a total of 28 photographs were submitted. All participants comfortably discussed each photograph, except in one instance when one participant who submitted more than five photographs decided to withdraw one photograph. Based on their descriptions of the experience of engaging in the photography activity and the use of photographs in the second interview, the main theme, Promoted reflection and richer dialogue, was identified. This main theme describes how the opportunity to take photographs over a period of time enabled participants to fully explore and reflect upon their experiences. Additionally, by using those photographs in the follow-up interview, it encouraged richer dialogue between the participants and the researcher.

\section{Main Theme: Promoted Reflection and Richer Dialogue}

For all of the participants, taking the photographs provided a greater sense of perspective of their experiences. Participants embraced the photography activity and found it to be beneficial as the reflection they experienced cultivated more awareness towards their caregiving role, their relationship with the care recipient, and their use of mobile apps for support. The photographs 
enabled the participants to describe their experiences and the meanings they ascribed to them in more detail than had been accomplished in the first interviews.

Participants expressed that by engaging in the photography activity, it promoted reflection of their caregiving experiences which they often do not get the chance to do. For instance, Abigail appreciated the opportunity afforded through the photography activity to reflect on her role as a caregiver as well as the impact of mobile apps on her caregiving experiences:

I was doing all these things that were in the pictures on a weekly basis anyways but I never actually got time to reflect on what I was doing until I took the pictures. I was like oh! I didn't even realize this was what I do every week. It gave me a chance to kind of reflect and give me an opportunity to see what I am actually doing... It kind of made me fully reflect.

It was evident that the photography activity allowed participants to elaborate on different aspects of their caregiving experiences that perhaps would have not been raised in a verbal-only interview alone. For example, as illustrated in the following exchange, Sophia recognized that the follow-up interview, which allowed for photograph-oriented discussion, encouraged richer dialogue - a result she did not anticipate. Sophia remarked, "I think we have gone into it a lot deeper than I thought I would." She elaborated that "some of the questions really made me touch base on it. Like anything in life, right? If it's not questioned, we really don't think about it. ... it's very reflective."

In the follow-up interview, participants discussed what was in the photograph and where, when and why they took the pictures, allowing them to reflect on a deeper level on the symbolic significance of the photographs. For Sierra, she stated that by having the opportunity to discuss her feelings and emotions in the photo-elicitation interview, it allowed her to explore her reflections in a more in-depth manner:

I think like for me, I like to talk through problems and I think when you're like reflecting on something within yourself, you don't always get the opportunity to talk about how you 
feel because like it's yourself. Like you're just talking with yourself so you're reflecting within yourself. And you don't get to. But for me, talking out loud helps me work through problems and I think that's why I was able to learn a lot more and explain myself like my vague idea more concise and clearly and get my point across easier when I was talking and I felt like I was able to share more of like how I felt like in words.

Participants also gained a richer insight into their emotions and feelings associated with their caregiving experiences. The photography activity appeared to encourage participants to reflect more deeply about their experiences as opposed to reflecting as part of a one-off interview. For instance, the photography activity enabled Cassandra to explore and reflect upon her experiences as a family caregiver as opposed to a professional caregiver like her aunt who is a Registered Nurse. Cassandra took a photograph of a page in a book (this photograph is not shown due to copyright infringement concerns) depicting a highlighted quote by Rath (n.d.): "You cannot be anything you want to be - but you can be a lot more of who you already are". She explained how even though she is not a "professional", she recognizes that she can still be a caregiver that provides quality care for her grandmother:

I literally just turned to one page, like a random page, and that was the quote that like came up. That was the first thing that I read because it was highlighted. And I thought it kind of related to my situation, right? So I just thought that yeah I'm not really a natural... professional caregiver and I'm not like the one that's always taking care of her and everything. And I can't be there but I am also like I am okay with it. Because I know that deep down, I can just be like there for her and just care for her.

For Sierra, by taking photographs to explain her experiences, she felt that she was able to see things she had always known in a new way. She further described how the photography activity encouraged her to see and reflect upon her experiences in a different light with regards to her experiences as a long-distance caregiver. As shown in the following dialogue, Sierra elaborated on the impact of being a caregiver on various aspects of her life, such as relationships with her mother, family and friends: 
I think just being able to like to reflect on my experience like in a different way... like seeing how things around me influence like caregiving for my mom like how little things. So like when I was away from my mom, I took some photos too like when I wasn't with her because I was trying to portray like the "being away from home" and stuff. That kind of like aspect of like caregiving from afar. And I think like it was beneficial to reflect on how it affects me when I am not at home too. So I think, that's something I don't often think about.

Furthermore, the use of photographs supported participants to talk about difficult areas of their caregiving experiences. In particular, Sophia opened up about her struggles with receiving peace of mind when her and her family made the decision to place her grandfather in palliative care when his health was declining: "I reflected on peace of mind like I wanted to feel that he was okay with what we did for him." She further explained how the photography activity promoted her to reflect in more depth on this challenging aspect of her caregiving experience and in doing so, she recognized that her and her family did what was best for her grandfather: "When I look at the photos and I look at the activity, I think what was available at that time. What we could have done for him to improve his quality. We did. I felt like we did it to our best."

In addition, the photography activity also helped participants realize and reflect upon their experiences with the changes that have occurred as a result of dementia. For example, Cassandra explained the feelings and emotions associated with navigating those changes as she discussed the meaning behind this following photograph: 


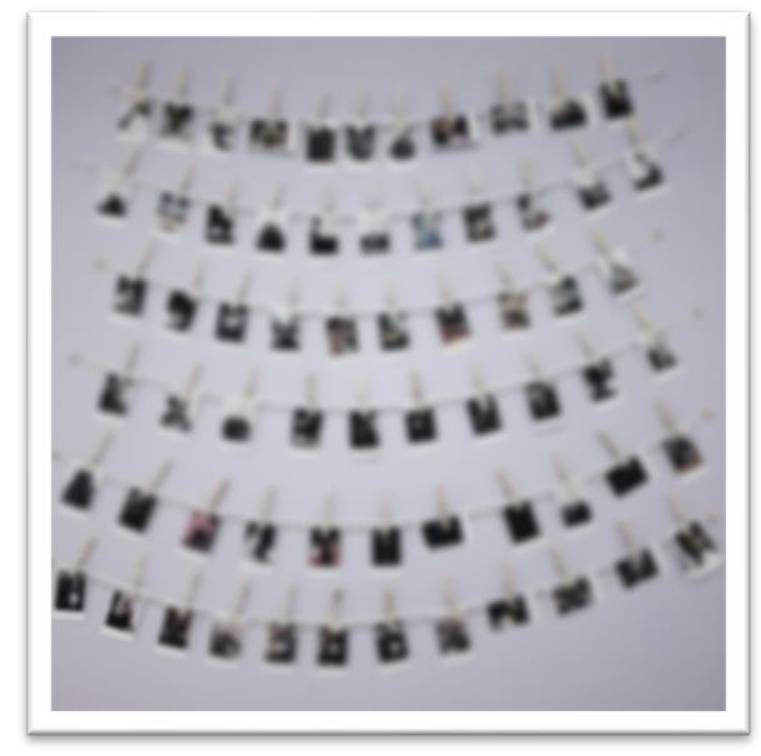

Photo 17. "Pictures represent memories and I blurred it; I just see myself kind of in her shoes" Taken by Cassandra

It's basically just pictures of a bunch of Polaroids and a few of my pictures really represents things that have happened in your life. So like memories and everything. And I blurred it, like I edited it, because it kind of symbolizes that she knows that she has children, she knows that she has grandkids and everything and family. And you know how pictures represent like memories right?... She doesn't really remember much of it. So let's say she looks at a photograph of something in her past, she will remember it... but like very vaguely. Like she'll forget... Well, when I look like at it, I just like see myself kind of in her shoes but not to, like be extreme, right? Because I don't really know what happens with her, but I see that when I take care of her, she really does get frustrated and just like not her usual self.

Participants recognized that although grief and loss is a natural part of navigating such a change, there is also an opportunity to acknowledge the celebration of who the person was, and who the person is now. For instance, Cassandra described the photography activity as a "time of reflection" about the impact of dementia on her grandmother and their relationship as well as to celebrate who her grandmother is now:

When I was little, I remember she would take me to school, get me dressed, and everything. And seeing how much that changed, it really affects me but you know, it's life, it happens. So we kind of just have to accept it and just like work with it... It makes me so much more thankful that she is still here with us and like she still remembers us. I 
mean one day, I know she will forget but just right now, we just like want to cherish her and everything.

In the same vein, Nadia also described how the photography activity promoted not only her reflection of, but also her appreciation of who her grandmother was, and who she is now:

It kind of makes you think. This whole activity is just you know I kind of remember her for how she was a few years ago or it was kind of, it was a gradual but kind of a quick decline... taking the pictures let's say five years ago, they might be completely different than what I'm taking now so that was kind of something where I mean I maybe felt a little saddened about but that's not, not too much. I mean I love who she is now and I loved who she was back then... I think it was nice to kind of look at her in different lights like this. I think it's nice to think about her like through this lens, through photography and everything like that.

With regards to user experiences with mobile apps, the photography activity and the follow-up discussion offered the opportunity for more in-depth exploration which appeared to give participants a new sense of appreciation of the impact mobile apps have in their caregiving role and its future use. For instance, Abigail asserted that she will now be more cognizant of her use of mobile apps and try to further leverage them in her caregiving role:

I am going to be thinking more about what I actually use on a day to day basis. It's kind of like created awareness so that way, before I would just do things and not think about it. But now, I am going to put more attention about what I am doing. I am actually, probably, going start to use apps more if anything and research different apps. Because I haven't, because I know the app store is very large so I am sure I can find one or two that are even more helpful.

Similarly, Nadia felt that the follow-up discussion encouraged her to reflect deeper on her mobile app use to assist with her caregiving activities and on how she could explore that aspect of her caregiving experiences further in the future:

It definitely got me to reflect on things and look at different options with mobile apps and technology that I just didn't even think about. I kind of just assumed it would be us with her, taking care of her and whatever. But there's also handy aids that are out there that you don't even know about. So that's definitely changed it. I didn't even think of it to be honest before we had these conversations. 
It was noted that the follow-up interview, somewhat expectantly, highlighted the aspects of their caregiving experiences and their use of mobile apps that were often overlooked. For instance, after reviewing the first transcript and gaining a better understanding of my discussion with Abigail, I began the follow-up interview inquiring about the social supports she has in her life as this was not discussed in detail in the first interview. As evidenced by the following dialogue, this discussion led Abigail to recognize an aspect of her caregiving experience that went unnoticed: using mobile apps to communicate and connect with family members to garner social support.

Interviewer: Okay, so you said you use Whatsapp to connect with your friends and family, would you say that using these apps, or other social network platforms such as Facebook, Twitter, Whatsapp, it helps you socialize with others without having to sacrifice caregiving in person?

Abigail: Oh yes, for sure! It helps very much. Especially with my sisters because I talk to them almost every day and I give them updates about my dad too on a weekly basis.

Interviewer: Is Whatsapp your go-to or do you use any other apps to...

Abigail: Whatsapp, Facebook messenger, these two are used very often.

Interviewer: Okay, so what I am hearing you say is these apps that we didn't talk about before...

Abigail: Oh my god, I didn't even think those apps for caregiving, yeah.

Interviewer: Yeah, I was reflecting about our previous discussion and you were talking about having difficulty to grocery shop and time away from your father and I made this link.

Abigail: I didn't tie that to dementia but I just realized that it could totally be important to that.

Further on in the interview when discussing a photograph she took, the same form of revelation occurred once again when Abigail recognized how the photograph helped her to acknowledge her use of banking apps to assist in caregiving activities which she never considered: "I am 
actually surprised right now at myself that I didn't even realize that I am using, that even the TD Bank app can tie into this." Sierra expressed similar sentiments as she recognized, through the sharing of photographs and dialogue, the apps that she used to address her caregiving needs that went unnoticed: "I only just realized this as I am talking to you and thinking about it... I have never thought about it before."

Overall, by participating in the photography activity, participants noticed that they were able to express feelings and experiences in a different yet deeper manner which required further thought and reflection on their part. The photographs also helped build rapport and trust between the researcher and participants by stimulating more in-depth discussions. In particular, there were many occasions where seemingly mundane images, such as trees in a backyard and take-out food, revealed complex narratives relating to the use of mobile apps in caregiving. The interaction between the photographs and the associated conversations was important in understanding what the participants wanted to express. As such, PEI facilitated richer dialogue to better understand the participant's message about their experiences. The use of photographs also prompted participants to talk about difficult or abstract concepts as well as gave them the opportunity to consider what they wanted to talk about at the interview. As a result, the photographs prompted emotional connections to memories, promoted in-depth reflection, and provided more meaningful accounts - which ultimately enhanced the richness of the narratives.

\section{Conclusion}

In this chapter, I have presented the findings of the study in which three main themes and the affiliated subthemes were identified that represented family caregivers' user experiences with using mobile apps in their caregiving role. Of note, I have compiled a complete list of the mobile apps and their descriptions that participants mentioned in the study in Appendix M. I have also 
presented the findings on how the use of PEI led to more comprehensive data through the promotion of reflection among participants and richer dialogue during the follow-up interviews. The overall findings provide a larger framework on how mobile apps should be conceptualized so that it is relevant to the complex nature of caregiving in the dementia context. In the next chapter, I present a discussion of the findings in relation to the current literature, the study implications, and the strength and limitations of the study. 
CHAPTER SIX

DISCUSSION, LIMITATIONS AND IMPLICATIONS 
"When creating content, be empathetic above all else. Try to live the lives of your audience."

\section{- Rand Fishkin}

\section{(American Author) \\ INTRODUCTION}

In this chapter, I present a discussion of the research findings and how these situate within the current literature and theoretical understandings of the family caregiver experience in the dementia context. In the first section, I address the main research question and the research sub-questions relating to user experiences of family caregivers that guided this study: What are the user experiences of family caregivers using mobile apps to assist in caregiving for their loved ones living with dementia in the community? (main research question); What are family caregivers' perspectives on the use of mobile apps to support caregiving activities?; What are the influences that impact the use of mobile apps for family caregivers?; How do family caregivers use mobile apps during their caregiving activities? In addition, I also comment on how the findings contribute to existing literature, and how the novel findings from this study can inform how family caregivers use mobile apps in their caregiving role. In the second section, I discuss the findings in terms of the research sub-question regarding photo-elicitation interviewing (PEI): What are family caregivers' experiences of using photographs as a conduit for explicating their user experiences? In this section, I explicate the impact of including participant-taken photographs in the interviewing process from the participants' perspective. At the end of each section, I outline the implications of the study findings. Lastly, at the end of this chapter, I discuss the strengths and limitations of the study. 


\section{PART I: MOBILE APP USER EXPERIENCES OF FAMILY CAREGIVERS}

Exemplifying the overarching theme, Connecting to support through mobile apps in my, your, and our lives, the findings reflected how and why participants used mobile apps to support the various challenges they faced in their caregiving role. The findings further revealed that the role of mobile apps often shifted from meeting the individual needs of the caregiver, to the care recipient, or to them as a dyad. Thus, highlighting how the impact of mobile apps for participants and their loved ones are inextricably linked.

In this section, I present a discussion of the three main themes and their affiliated subthemes: (1) Adapting mobile apps to meet individual needs of the dyad; (2) Minimizing the impact of the condition on the person and the family; and (3) Determining the effectiveness of mobile apps. Under the first theme, the discussion revolves around family caregivers' experiences of adapting available mobile apps to meet the individual needs of the care recipient as well as themselves in three main areas: connecting with others, assisting with care activities, and organizing everyday living. The concept of biographical disruption is explored under the second theme to better understand how and why participants are using mobile apps to minimize the impact of the condition on the care recipient as well as their families as a whole. Lastly, for the third theme, the discussion focuses on the areas that promoted uptake and presented as barriers towards continued use of mobile apps as well as how the findings are positioned within existing research regarding technology use and acceptance.

Ultimately, the discussion illustrates the complex, multifaceted and nuanced caregiving experiences of participants as well as presents rich information on family caregivers' perspectives, the influences that impact their use of mobile apps, and how and why mobile apps are used as a form of support in participants' caregiving roles. In this section, I conclude with the 
implications of these findings in relation to education, practice, future app development, policy, and research.

\section{Adapting Mobile Apps to Meet Individual Needs of the Dyad}

\section{Connecting with Others}

In this study, social support has been described as a central component of the lives of participants and their family members living with dementia. Participants used various social media and communication apps to communicate and connect with family, friends, and other caregivers. This finding is expected given that increased social support has been found to reduce social isolation and depression (Hensel, Parker-Oliver, \& Demiris, 2007; Mickus \& Luz, 2002), have a positive impact on health and life satisfaction (Hensel et al., 2007; Mickus \& Luz, 2002), as well as increase the capacity for resilience-building for family caregivers of persons living with dementia (Parkinson, Carr, Rushmer, Abley, 2016). Moreover, the importance of family caregivers having the ability to connect with family, friends, and other caregivers in an accessible manner through mobile apps are also demonstrated in other studies involving family caregivers. For instance, in a study exploring the user experiences of family caregivers of children with acute lymphoblastic leukemia who used a mobile app designed by researchers in China, Wang and colleagues (2016) found that participants valued the function of the app where they were able to connect and share with other caregivers. By doing so, the participants described being able to find social support and gain confidence in caring for their children. The participants also suggested adding communication tools such as QQ and WeChat, the most popular social networking platforms in China, to extend their access of social support to family and friends. Similarly, people living with Cystic Fibrosis (CF) in a mixed-methods study 
conducted by Hilliard and colleagues (2014) also contended that social networking apps can increase social support opportunities for families and friends of people living with CF.

In particular, participants described connecting to other family members to coordinate care and receive support as well as communicating with the care recipient as a crucial element of their caregiving experiences. Family contact provided an important source of social support for participants and their loved ones living with dementia. In response, participants in this study described how mobile apps facilitated communication and contact in cases where distance is traditionally a barrier to face-to-face interaction. They also emphasized the benefits of these apps as they allowed for the ability to give and receive visual cues not afforded through an audio-only telephone call. For participants, the video component of the communication enhanced the quality of the social interaction and increased the perception of social presence. Indeed, researchers have found that technology that enables both visual and verbal communication to be more beneficial when compared to telephones (Hensel et al., 2007). Mobile apps, such as FaceTime ${ }^{\mathrm{TM}}$, can not only accommodate for spoken words but also visual nonverbal cues such as facial expressions, gestures, and posture. Physical appearance cues, such as clothing and hairstyle, as well as one's surrounding environment can also be communicated (Hensel et al., 2007). As a result, a greater sense of social presence is achieved. These findings have significant parallels with Short and colleagues' (1976 as cited in Hensel et al., 2007) Social Presence Theory which posits that different telecommunication technology accommodates varying degrees of social presence, thus influencing the interaction between individuals. They found that video with audio communication has a higher degree of social presence than audio-only communication due to its capacity to transmit visual nonverbal cues. 
In the context of caregiving, the visual component allowed participants to better evaluate how the care recipient was doing and vice versa. They felt as though it was almost like being in the same room as the care recipient. The video component enabled participants to feel a part of the other person's environment and to experience the setting in ways that would not be possible in an audio-only communication method. This is line with previous research which has demonstrated communicating using technology using video and audio communication, such as videophones, was valuable for care recipients and their family caregivers as they enhanced the quality of the social interaction (e.g. Mickus \& Luz, 2002; Tsai \& Tasi, 2010). In a study conducted by Sävenstedt and colleagues (2003) which explored the use of videophones by residents living with dementia in a nursing home, findings showed that the interaction achieved by the videophones increased the attention and focus of residents as well as gave family caregivers a greater sense of involvement. Moreover, in a case study Hensel and colleagues (2007) conducted involving a nursing home resident and a family caregiver, the videophone technology was found to enhance social presence as the participants expressed that the experience was almost like being in the same room. Additionally, the participants highlighted the benefit in using the videophone for assessing how the other was doing. These experiences support findings by Demiris and colleagues (2008) as participants appreciated being able to see their family members' facial expressions, to have sense of closeness, and to obtain the opportunity to assess the care recipient's condition. Overall, participants found this form of communication through videophones more personal and comforting as well as contributed positively in reducing their feelings of isolation and loneliness (Demiris et al., 2008).

The study findings suggest that, with the added visual component, there are additional benefits of video-based communication as compared to audio-only communication, particularly 
enhanced perceptions of social presence. This in turn helped participants maintain meaningful relationships with other family members and the care recipient. Of note, research has shown that the quality of family relationships and social support can impact the family caregivers' desire to institutionalize the care recipient (Spitznagel, Tremont, Davis, \& Foster, 2006). Perhaps given that the current study revealed that communication apps can have many benefits that ultimately enhance the quality of family relationships and promote social support among family members, further exploration is required to examine if the use of communication apps can influence the outcomes of the care recipient (e.g. a family caregiver's decision to institutionalize). It is also important to recognize that in contrast to other studies, participants in this study used mobile apps that were free of cost and easily accessible to them through their mobile device. On the other hand, videophones - although described as low-cost - are more costly than mobile apps (e.g. can cost up to $\$ 150$ according to Demiris et al., 2008). As such, mobile apps have an additional benefit of being more affordable than other alternatives which can be valuable for family caregivers.

Furthermore, it was found in this study that mobile apps helped participants facilitate their loved ones' engagement in social activities despite changing functional abilities and thus, preventing feelings of loneliness and social isolation. Researchers have indicated that persons living with dementia tend to be isolated with few opportunities to communicate and to form connections with others (Yasuda, Kuwahara, Kuwahara, Morimoto, \& Tetsutani, 2013). Hence, feeling connected to family, friends and the wider community is significantly valuable for this population (Han, Radel, McDowd, \& Sabata, 2016; Roland \& Chappell, 2015). Given the many benefits, social activities have been found to be strongly linked to well-being among persons living with dementia as well as their family caregivers, suggesting the need for greater 
accessibility to social opportunities (Giebel \& Sutcliffe, 2018; Giebel, Challis, \& Montaldi, 2016). Thus, providing opportunities to communicate with family and friends via mobile apps is one of the most important interventions for persons living with dementia (Kerkhof, Bergsma, Graff, \& Droes, 2017). Aligned with this, participants described using social media and communication apps to help the care recipient stay connected with others, particularly with family members and friends. These experiences are similar to those who participated in Asghar and colleagues' (2018) study on exploring the experiences of persons living with dementia who use different assistive technologies for performing their daily activities. The participants believed that assistive communication apps were crucial as it decreased their loneliness and resulted in more socialization and interaction with family and friends. Notably, in another study conducted by Kerkhof and colleagues (2017), participants, which comprised of persons living with dementia and their informal caregivers, particularly valued apps that could stimulate social contact and communication between them and their caregivers. These apps were reported as helpful in giving persons living with dementia access to the world and thus, provided them a sense of social inclusion. Interestingly, the authors emphasized that apps such as Skype ${ }^{\mathrm{TM}}$, FaceTime $^{\mathrm{TM}}$ and WhatsApp ${ }^{\mathrm{TM}}$ - apps that participants in the present study described using - are desirable for persons living with dementia and their caregivers given that it supports them in being socially active. Overall, the findings discussed in this subtheme add to a growing body of research that demonstrates the benefits of mobile apps in supporting persons living with dementia and their family caregivers in garnering social support from family, friends, and other caregivers. 


\section{Assisting with Care Activities}

In this study, participants indicated that the changing functional and cognitive capacity of the family member living with dementia had an impact on their daily activities. Thus, participants began taking on more responsibility for daily activities, and their loved ones relied increasingly on their help. Given the increase in responsibilities, participants turned to mobile apps to assist with their caregiving role. The findings shed light on how participants used mobile apps to assist with ADLs and IADLs of their loved ones through three types of apps: (1) reminder apps to help with managing medication administrations, making and keeping appointments with healthcare professionals, and meeting personal care needs; (2) grocery-and food-delivery apps to meet grocery shopping and nutritional needs; and lastly, (3) ride-sharing and car-renting apps to address transportation needs. Of particular interest and relevance is the finding that the use of mobile apps mainly focused on initiating the act of performing ADLs and addressing IADLs. For example, reminder apps were the only form of apps that helped participants with ADLs by having the mobile app prompt them when these tasks needed to be completed so it will not be forgotten. This was especially important when their loved ones are at a stage of dementia where they have difficulty in verbally communicating their self-care needs. In this context, mobile apps were used to prompt and help participants initiate the care activity. This is supported by previous research that indicated deficits in the initiation of activities can be relatively easily addressed with the use of technology, in providing reminders and thus effective triggers (Arntzen, Holthe, \& Jentoft, 2016; Giebel \& Sutcliffe, 2018).

Furthermore, mobile apps used by participants in this study predominantly focused on addressing care activities related to IADLs. The focus on IADLs as opposed to ADLs may be due to the complexity of the former. Researchers explained that completion of IADLs often 
require a complex set of behaviours and cognitive functions such as organizational ability, future planning and prioritizing, and tend to decline in the early stages of dementia (Giebel, Sutcliffe, \& Challis, 2015; Giebel, Challis, \& Montaldi, 2015; Ha \& Kim, 2014). Thus, assistance from family caregivers in this area of daily activities are prevalent and particularly appropriate for mobile apps as evidenced by the findings of the current study. The specific areas of IADLs that participants used mobile apps for assistance were found in the literature to be prominent tasks that persons living with dementia face difficulties with. In a study by Pérès and colleagues (2008), using transport and adherence to medications were significantly impaired in persons living with dementia. In an ecological study involving direct observation, Bier and colleagues (2013) found grocery shopping and meal preparation to be more impaired when compared other activities. Notably, the findings of the current study correspond to results of earlier studies wherein persons living with dementia and their family caregivers reported the following areas in which assistance for the care recipient was most frequently required: grocery shopping, food preparation, medication management, and transportation (Asghar et al., 2018; Giebel, Sutcliffe, \& Challis, 2017; Miranda-Castillo, Woods, \& Orrell, 2013; Van der Roest et al., 2009).

Importantly, the findings unveiled that mobile apps can effectively assist family caregivers with IADLs such as ordering culturally-appropriate meals. A salient example is how Abigail used mobile apps to order culturally appropriate meals for her father when she was unable to cook for him. This was important as her father was described to be a "picky-eater". Researchers have indicated that improving food intake among persons living with dementia is imperative as they are likely to have poor nutritional states and thus, they should be offered food that caters to their culture and preferences (Hanssen \& Kuven, 2016; Kelly, 2016; Prizer \& Zimmerman, 2018). Given this emphasis, food-ordering apps assisted Abigail in meeting her 
father's nutritional needs. Another prevalent example is the use of reminders to assist with medication management, which was common in the current study. In a qualitative study conducted by El-Saif and colleagues (2018), they also found that the use of reminder apps were embraced by family caregivers when managing medication adherence challenges in their relatives living with dementia. Family caregivers in another qualitative study by Ruggiano and colleagues (2019) also reported using mobile apps to manage appointments and medications for their loved ones living with dementia. As such, there is growing evidence, consistent with the current study, that family caregivers are presently use mobile apps to assist in care activities.

Overall, addressing these areas of daily activities is of particular importance as research has shown that poorer IADL and ADL performance are significantly associated with reduced well-being and quality of life in persons living with dementia and their caregivers as well as heightened levels of caregiver stress and burden (Giebel et al., 2014; Giebel, Challis, \& Montaldi, 2016; Giebel \& Sutcliffe, 2018). In fact, researchers suggested that the use of technology in assisting with ADLs and IADLs could reduce the responsibilities and burden placed on the caregiver (Giebel \& Stucliffe, 2018; Klimova et al., 2018). As described by the participants in this study, by using appropriate mobile apps, they can offer the necessary support they require to effectively provide assistance in their loved ones' daily activities. Consequently, reinforced by the literature, supporting care recipients in their daily activities can result in positive outcomes for both the person living with dementia and their family caregivers.

\section{Organizing Everyday Living}

Participants described the challenges of occupying multiple roles in addition to their caregiver role. Mirroring the findings of this study, research has indicated that adult child caregivers are typically at a stage in their lives where they often struggle to divide their time and 
resources between caregiving for their parents or grandparents and their other roles and responsibilities, including being a spouse, parent, employee and student (Bastawarous, Gignac, Kapral, \& Cameron, 2015; Cohen, Cook, Kelley, Sando, \& Bell, 2015; Wang, Shyu, Chen, \& Yang, 2011). In fact, the needs of adult child caregivers who may simultaneously balance work, child caregiving, and school obligations are distinct from those of spousal and other types of informal caregivers (Cohen et al., 2015). Unsurprisingly, many adult child caregivers experience challenges in reconciling the multiple roles they hold, and this often results in them sacrificing their leisure time, keeping a tight schedule, and reducing work hours or withdrawing from the labour market overall in order to maintain their caregiving role (Wang et al., 2011).

The manner in which participants in this study described their understandings of their experiences with holding multiple roles is similar to the tenets of the depletion/role strain perspective. It is contended in the depletion/role strain perspective that individuals have a finite amount of time and resources that can be allocated across roles (Bainbridge, Cregan, \& Kulik, 2006; Rozario, Morrow-Howell, \& Hinterlong, 2004). This view underscores the notion that these fixed resources force trade-offs whereby a commitment to one role will reduce the resources that could be allocated to another (Bainbridge et al., 2006; Rozario et al., 2004). In this lens, an individual can experience stress created by the conflicting demands for limited resources, which impacts the functioning in each role (Bainbridge et al., 2006). This principle resonated with participants as they described the fact that they have a fixed amount of time and energy. They also recognized that the multiple roles they held are inextricably intertwined and thus, if one role is not well organized and managed, the impact can spill-over into the other roles. As such, participants described how they turned to mobile apps for assistance in organizing the tasks and responsibilities associated with their multiple roles. In doing so, mobile apps enabled 
participants to exhaust less of their time and resources into their other roles by helping them organize their daily activities as well as by offering them the ability to complete tasks on the go (i.e. during commutes). As a result, this left them with more time and energy which can then be allocated to their caregiving role.

\section{Summary}

Ultimately, the findings in this theme highlight an important message, which is threefold, about the experiences of family caregivers who adapt mobile apps to meet the needs of the dyad. First, given the potential for social isolation associated with caregiving and dementia described by participants, it is unsurprising that app-based socialization and connection with other people, including family, friends and other caregivers, was a major focus for all participants. Second, the use of mobile apps greatly supported participants in assisting with their loved ones' ADLs and IADLs, which they described as helpful in reducing the burden placed on them. Lastly, unique to the adult child caregiver population are the multiplicity and combination of the roles they occupy. This finding, in conjunction with past literature, indicates that adult child caregivers may require support with managing the multiple demands (Bastawarous et al., 2015). Bearing this in mind, participants in this study found mobile apps as an effective method to support the management of multiple roles and their associated responsibilities, across generations of caregivers.

\section{Minimizing the Impact of the Condition on the Person and the Family}

In this main theme, participants in the study described how they used mobile apps to respond to the inevitable disruptions and changes that came into their lives as a result of dementia. Dementia can cause disruption and change in not only the individual's life, but also the lives of their family members (Hasselkus \& Murray, 2007). For the individual, dementia can 
impact their ability to continue involvement in familiar and meaningful activities. This form of disruption on an individual level can be best understood by Bury's (1982) concept of "biographical disruption" which illustrates how one's sense of self and social world is affected by chronic illness. Bury (1982) postulated that chronic illness can cause people to experience a discrete rupture in their ability to enact familiar routines and activities as "the structures of everyday life and the forms on knowledge which underpin them are disrupted" (p. 169). In the first subtheme, participants highlighted the disruptions caused by the condition on their family member's ability to maintain involvement in meaningful activities. Nonetheless, recognizing the importance of meaningful activities in order to retain one's personhood, participants in the study used mobile apps to promote their loved one's engagement in previous interests, hobbies, and activities. The concept of personhood is explored under this subtheme with specific focus on the intersection of the findings with the writings of Kitwood (1997) and other research pertaining to meaningful activities.

This study moved beyond exploring disruptions at an individual level as the findings also showed that while the condition is not their own, its presence can cause disruption in the lives of the family as a whole and force major changes in the routines and traditions that constituted as family life. These experiences support an earlier finding by Zimmermann (2013) where the author found that adult child caregivers for persons living with dementia also experience a disruption in their lives as they witness the progression of the condition on their loved ones. Participants in the current study underwent what Phinney and colleagues (2013) referred to as a "collective biographical disruption" which is built on the work of Bury (1982) to further explicate how biography can be impacted on a family level as the focus of biographical disruption is largely on the individual and not on the family as a whole. In the second subtheme, 
participants discussed how they strove to minimize the collective biographical disruptions by using mobile apps to help maintain shared family routines and traditions.

\section{Promoting the Care Recipient's Personhood}

An enlightening finding from this study was that participants asserted the importance of seeing their relatives living with dementia first and foremost as people. Participants recognized that their loved ones are experiencing biographical disruption caused by dementia which can affect their sense of identity and how they see themselves (Bury, 1982). Despite the context of this study and participant groups being very different, a close comparison with the biographical disruption described by Bury can still be drawn. For instance, Bury identified the need to try and maintain normal activities as a key feature of biographical disruption but this normality was altered for his participants given that activities had to be restricted in the face of limited ability due to chronic illness. In the same way, in the face of declining cognitive and physical abilities of the care recipients, participants in this study spoke candidly of how they addressed those biographical disruptions by celebrating who the care recipients were, and who they are now. Additionally, they understood the need to maintain a sense of identity and normality for their loved ones and felt that this can be best achieved through engagement in meaningful activities that are based on their loved one's needs, preferences, values, beliefs and life history. Closely aligned with Bury's work, Atchley (2000, as cited in Menne, Kinney, \& Morhardt, 2002) further explained this experience of needing to maintain involvement in previous activities in his continuity theory. Atchley argued that "people will attempt to preserve and maintain the longstanding social patterns of living and coping that they identify as being uniquely their own" (p. 143). In other words, people will strive to continue to engage in activities in which they view 
are a part of their identity, which is consistent with the study findings as illustrated through the narratives of the participants.

The notion of meaningful activities as central to one's well-being in persons living with dementia is well-documented in dementia research. Activity theory has long posited that older adults who remain engaged in activities, particularly meaningful activities, experience improved psychological and physical well-being than their counterparts who are less involved (Masbach, Mace, Clark, \& Firth, 2017; Roland \& Chappell, 2015). In fact, there is a substantial body of evidence linking involvement in meaningful activities and positive health outcomes for both persons living with dementia and their family caregivers (see, for example, Genoe \& Dupuis, 2014; Phinney, Dalke, \& Purves, 2007; Roland \& Chappell, 2015). What encompasses activity is diverse, comprising of household chores, recreation, work-related endeavours and social involvement (Vernooij-Dassen, 2007). In contrast, what makes it become meaningful is when the activity promotes a sense of pleasure, connection, belonging, and identity (Masbach et al., 2017; Vernooij-Dassen, 2007). It is important to note that persons living with dementia can also, and often do, find meaning in activities previously considered as ordinary (Genoe \& Dupuis, 2014). However, escalating cognitive and physical impairments experienced by the person living with dementia can restrict their engagement in meaningful activities (Roland \& Chappell, 2015; Vernooij-Dassen, 2007). Several studies have found that community-dwelling persons living with dementia and their informal caregivers reported having a lack of meaningful activities for persons living with dementia to participate in as a prominent unmet need (Black et al., 2013; Miranda-Castillo, Woods, \& Orrell, 2013; Van der Roest et al., 2009).

In this study, the participants reconciled the changes associated with dementia, drew upon the support of mobile apps, and subsequently felt more in control of their situations. Participants 
recognized the importance of offering activities through mobile apps that reflected the care recipient's past experiences, interests, hobbies, and remaining capabilities. In doing so, they provided a sense of continuity with their previous lifestyle and long-held beliefs for their loved ones by allowing them to feel that they can still do things that matter most to them; as a result, care recipients were able to sustain their sense of self (Han et al., 2016; Phinney et al., 2007). Specifically, participants promoted the involvement in meaningful activities for care recipients by using mobile apps that allowed care recipients access to previous interests and hobbies, such listening to music $\left(\right.$ YouTube $\left.^{\mathrm{TM}}\right)$, watching TV shows (Netflix ${ }^{\mathrm{TM}}$ ), enjoying nature (gardening apps), and being creative (colouring and puzzle apps). These findings mirror the results from a study by Kerkhof and colleagues (2017) wherein participants who are persons living with dementia and informal caregivers indicated that they preferred using apps that matched their personal interests and hobbies, such as creative activities (e.g. knitting and drawing), being in nature (e.g. sitting in the garden), listening to music, and watching TV. It is apparent that there is emerging evidence suggesting that both persons living with dementia and their caregivers are not only receptive, but actively engaging in the use of apps for completing meaningful activities.

Participants specifically chose to use mobile apps as a strategy to create opportunities for the person to be involved in meaningful activities as it made the activities easier for them to complete. This was particularly evident when Cassandra chose a colouring and gardening app for her grandmother that she described was easy to use and follow. By doing so, her grandmother was able to continue to enjoy the meaningful activities that she used to, such as colouring and gardening. Phinney (2006) describes this strategy for supporting involvement in meaningful activities by family caregivers as "reducing demands" whereby family caregivers create an environment that affords involvement by making the activities easier. Family caregivers 
implement this strategy in order to support their remaining abilities (Phinney, Dahlke, \& Purves, 2013) and to encourage the loved one to continue to enact what is most important for their sense of personhood (Phinney, 2006). In addition, participants in the current study sought help from mobile apps and adjusted the activity in order for their loved ones to continue to enact the activity. Although the nature of the activities was different (for example, in the case of Cassandra's grandmother, she is gardening virtually instead of completing it in person), its meaning and value were retained. The act of seeking help and altering the activity is also described in a study by Genoe and Dupuis (2014) wherein persons living with dementia sought help from family, friends, and formal service providers as well as altered their activities to match their new capacities in order to prolong engagement in meaningful activities. Other strategies reported in the literature include reminders, instructions, and accompanying the person living with dementia to ensure success in the activity (Phinney, 2006). The type of strategies in which family caregivers would enact to promote successful involvement in meaningful activities using mobile apps is an area that would warrant further exploration.

The findings further revealed that family caregivers have specific knowledge of their relative living with dementia that can enable them to consider their previous interests, hobbies, values and beliefs when promoting engagement in meaningful activities for the care recipient. Participants were instrumental in supporting activity engagement and as the findings suggest, activity can also be especially meaningful when it is shared between the care recipient and their family caregiver. In the instance where Nadia described enjoying music through YouTube $^{\mathrm{TM}}$ with her grandmother together, it demonstrates how an activity can become even more meaningful through togetherness. Nadia indicated that by engaging in this form of activity together that they both felt meaningful had a positive impact on their relationship, supporting the 
argument that meaningful activity enacted by the family caregiver and care recipient can contribute to higher levels of relationship satisfaction and happiness (Genoe \& Dupuis, 2014; Roland \& Chappell, 2015). In addition, the findings indicated that participation in meaningful activities can also positively influence one's sense of self-worth. For instance, by using mobile apps to colour or to complete puzzles, Cassandra stated that it gave her grandmother a sense of confidence and self-worth, and particularly, the opportunity to feel good about herself. Overall, by successfully involving their loved ones in meaningful activities, participants identified many positive outcomes, such as a strengthened bond with each other, increased feelings of self-worth, independence, and pleasure, as well as enhanced sense of continuity, stability and identity.

These experiences illustrated by participants can be best understood through Kitwood's (1997) description of personhood. Central to personhood is the recognition that the person living with dementia has a self with a range of psychosocial needs including comfort, attachment, inclusion, occupation, and identity (Kitwood, 1997). Involvement in meaningful activities is crucial to personhood because individuals need to be more than simply occupied but engaged in past and current interests and sources of fulfillment and pleasure (Fazio, Pace, Flinner, \& Kallmyer, 2018). Acknowledging personhood involves recognizing the needs, desires, emotions, personality, and life story of the individual (Palmer, 2013), and this was particularly salient throughout the narratives presented by the participants. In fact, promoting their loved one's sense of personhood was the predominant story line among participants in this study when discussing the use of mobile apps for continued engagement of their relative in meaningful activities. A sense of continuity is critical in establishing personhood (Perry \& O’Connor, 2002) and as such, participants identified retained abilities of their loved ones and implemented situations that encouraged them to do as much as they could do. This was demonstrated by their use of mobile 
apps that required minimal effort on the part of the care recipient in order to become involved in a meaningful activity. People define themselves "in relation to their long-standing patterns of doing" (Phinney, Dahlke, \& Purves, 2013, p. 364) and by engaging in meaningful activities, participants believed that their relative was able to retain a sense of their personal identity as "identities are closely tied to what we do" (Christiansen, 1999, p. 549 as cited in Phinney et al., 2007). Consistent with other studies (e.g., Chung, Ellis-Hill, \& Coleman, 2017; Hasselkus \& Murray, 2007; Phinney et al., 2013), participants used mobile apps as a form of support and assistance in engaging their loved ones in meaningful activities which addressed many of their relative's psychosocial needs, such as comfort, pleasure, inclusion, occupation and identity - and by addressing those needs, it ultimately promoted a sense of personhood for the care recipient. The ability of the person to maintain personhood is not just reliant on their own cognition, but those around the individual also have a duty to support their personhood (Kitwood, 1997). Indeed, it is thought that personhood is bestowed by others "in the context of relationship and social being" (Kitwood, 1997, p. 8). Participants in this study illustrated how, regardless of their loved ones' level of dependency or cognitive impairment, they ensured that their relative remained actively involved in meaningful activities by reconciling the reality of their condition and the consequent changes. Participants believed that actively engaging in meaningful activities that preserved feelings of identity and pleasure was important to promoting their loved ones' sense of personhood and quality of life. By doing so, it ultimately contributed to feelings of a meaningful and normal life (Genoe \& Dupuis, 2014). The findings of the current study provide further support for the notion that involvement in meaningful activities can promote the personhood of the person living with dementia, and that family caregivers play an important role in helping maintain this kind of involvement. More importantly, the findings also 
expand on this existing work by describing how family caregivers do this with the help of mobile apps.

\section{Maintaining Family Routines and Traditions}

Davies and Gregory (2007) contended that research tends to view the persons living with dementia and the family as "individuals living in parallel life spaces" (p. 481) rather than considering the unified history and context of the familial relationship. Phinney and colleagues (2013) also argued that much of the findings from research have not been situated in a family context. In fact, few authors have considered the concept of biographical disruption in a family context. Exceptions include some studies that have specifically explored the biographical disruption experienced by family caregivers (Aasbø, Solbrække, Kristvik, \& Werner, 2016; Hasselkus \& Murray, 2007; Hudson et al., 2016; Phinney et al., 2013). What is unique about the present study in contrast to the work conducted by other researchers on biographical disruption is the exploration of the family caregivers' experiences of navigating those disruptions that occur at a family level using mobile apps.

Specifically, in the face of disruptions that are caused by dementia on the family unit, participants illustrated how they strove to maintain long-standing family routines and traditions by using mobile apps to facilitate, for example, the continuity of celebrating Eid and birthdays as well as capturing memories through photographs. The mobile apps they used helped them maintain their shared narrative as a family, as well as the routines, traditions, and practices that constituted as who they are as a family. Using the family systems perspective to further understand this experience on a family level, it is evident that families can experience collective biographical disruption as the routines and traditions that constituted as family life can begin to become difficult to uphold as a result of dementia (Phinney et al., 2013). Thus, families will try 
to overcome the disruption by doing what they can to maintain those routines and traditions to sustain a sense of shared family identity (Phinney et al., 2013). The promotion of continuity and normality by family caregivers through continued involvement in family routines and traditions as seen in this study is also a salient aspect in several studies involving family caregivers. For instance, Phinney and colleagues found in their study with persons living with dementia and their family caregivers that family identity can be sustained through activity whereby participants would continue to enact on important family habits and practices in order to maintain a shared sense of who they were as a family. Similarly, other studies involving spousal caregivers and their care recipients found that they believed it was important to continue to engage in previous interests and rituals which had always characterized them as a couple in order to sustain their relationship (Aasbø et al., 2016; Hellström, Nolan, \& Lundh, 2007). In another study that consisted of not only spousal caregivers but also adult child caregivers conducted by Hasselkus and Murray (2007), the findings also exemplified how participants were trying to navigate the disruptions caused by dementia by continuing to engage in long-established practices to maintain a sense of continuity in their lives.

\section{Summary}

In conclusion, the findings in this theme suggest that disruptions can occur on an individual and family level as a result of dementia. The study first drew from Bury's work on biographical disruption to understand the experiences of living with dementia and the associated disruptions for the individual, but then expanded to Phinney's research on collective biographical disruption to further explore disruptions that occur within families. Most salient in the participants' stories was how they addressed those disruptions through continued involvement not only in meaningful activities for the care recipient, but also in shared routines and traditions 
for the family as a whole. Participants also stressed how mobile apps helped harmonize the previous identity with the new identity that emerged from the biographical disruption process on an individual and family level. For participants, this meant that they were able to maintain a sense of personhood for the care recipient, as well as a sense of normality and stability for their family. Considering the findings, it is worth noting that developers should consider the enhancement of personhood and the continuity of family practices during app design. Additionally, health and social care professionals could also assist family caregivers by providing activity strategies and support resources, such as mobile apps, for continued engagement of their relative in meaningful activities. This study adds to the current state of knowledge on the experiences of living with dementia and the resultant disruptions it has on the care recipient and the family, as well as how family caregivers can leverage mobile apps to manage those disruptions. The findings further contribute to the theoretical understanding that mitigating biographical disruptions on an individual and family level can be achieved through the use of technology, such as mobile apps, which facilitates a return to one's previous identity or a coming to terms with one's new situation.

\section{Determining the Effectiveness of Mobile Apps}

\section{Identifying Useful Functions of Mobile Apps}

In this study, participants outlined several characteristics and functions of mobile apps that they identified as useful in addressing the practical, emotional and relational challenges in their caregiving role, including simplicity, easy navigation, and interactivity and integration. Additionally, when it came to what functions best facilitated continued use of mobile apps for their loved ones living with dementia, participants also emphasized simplicity and easy navigation. This finding is congruent with Rosenberg and colleagues' (2012) study which 
contended that technology needed to be simple to use if a person living with dementia were to be able to use it. They elaborated that this meant a lack of demands on the user and minimal chain of actions from the user required.

Of particular note is that many of the characteristics and functions voiced by this study's participants echoed the preferences of participants of other research that investigated user experiences with mobile apps. Perhaps most universal is the desire for simplicity and easy navigation wherein the app is easy to use and required low effort. For example, after examining the usability of existing mobile apps for diabetes, depression, and caregiving through interviews and direct observation, Sarkar and colleagues (2016) also recommended design features of apps to enhance usability of an app similar to those echoed by the study participants. Some design features include the "use of simple language supplemented by graphics through out; reducing the number of screens for completion of each task; and reducing manual data entry as much as possible" (p. 1424). Additionally, Peng and colleagues (2016) conducted a qualitative study to explore the user perceptions of mHealth apps and found that ease of use and simplicity are top desired features among participants that encouraged continued use of mHealth apps. Goodwin and colleagues (2016) also found that their participants who are patients with mental health conditions discussed the significance of apps being accessible, self-explanatory and simply laid out. Other studies (e.g. Dennison, Morrison, Conway, \& Yardley, 2013; Hilliard et al., 2014; Middelweerd et al., 2015) echoed similar sentiments as their participants asserted the importance of apps to be simple and require low effort. It is important to point out that the participants in these studies are predominantly general users of mobile apps as well as patients with chronic and non-chronic conditions, not always family caregivers. As such, the literature suggests that these desired qualities are nearly universal and should be applicable to most mobile app designs. 
A paucity of literature exists in terms of the interactivity and integration component of mobile apps. The desire for interactivity was a commonality found in a study by Peng and colleagues (2016) where their participants expressed interest in the social networking features and the sharing feature of apps as sharing information with family and friends increased informational and emotional social support from them. Moreover, a finding from the current study that is distinct from the literature is the area of integration when understanding factors that influence the usefulness of a mobile app. In the context of this study, integration meant the ability for a mobile app to be integrated and available in all forms of mobile devices, such as smartphones, tablets, and laptops. This integration was described to be particularly important as it meant that participants could use a specific app on whichever device that is suitable at the time and it also provided the opportunity for participants to use an app in different settings. For example, being able to use an app to complete tasks on a smartphone while commuting on the bus and then using the same app afterwards on a laptop once the individual has arrived home. Of note, these findings serve to complement and supplement the research of others as the literature provides additional characteristics not mentioned in this study, such as appropriate text sizes, a help button that is accessible and clear, and the use of warm colours (Kerkhof et al., 2017, p. 2021).

The participants' experiences with identifying useful functions of mobile apps reflect what embodies the concept of usability. Usability has been well-documented in the literature as one of the main factors that can determine the success of mobile apps (Zapata, FernandezAleman, Idri, \& Toval, 2015). According to the International Organization for Standardization (ISO; 2018), usability is understood to be the ability to use a product with effectiveness, efficiency, and satisfaction in a specific context of use. Effectiveness describes the completeness 
with which users achieve specified goals, efficiency refers to the resources (e.g. time, effort, costs) used in relation to the results achieved, and lastly, satisfaction pertains to the extent to which the user is satisfied as a result from actual use meeting the user's needs and expectations (ISO, 2018). In the context of this study, participants discussed factors of efficiency (e.g. simplicity and easy navigation wherein they ultimately wanted apps that required minimal resources) that promoted the effectiveness (as evidenced by participants achieving their goals when using apps to assist with caregiving) and the satisfaction (as demonstrated by participants stating that the apps met their needs which led to app adoption and continued use) towards the apps they use. With this understanding in mind, the findings further support existing evidence that usability is important to consider when designing and developing apps for this population. Overall, the findings and previous research (e.g. Zapata et al., 2015) suggest that increased usability of an app can lead to higher levels of adoption and continued use.

Moreover, after further examination, it is evident that the categories of useful functions identified by participants closely resemble constructs within prominent theories and models, such as the Technology Acceptance Model (TAM) (Davis, 1989) and the Unified Theory of Acceptance and Use of Technology (UTAUT) (Venkatesh et al., 2003), that predict technology acceptance. For instance, simplicity and easy navigation are related to the perceived ease of use (degree to which people believe that using a particular system would be free of effort) and the effort expectancy (degree of ease associated with the use of the system) construct in TAM and UTAUT respectively, which are used to predict one's intentions to use a piece of technology in these theories. While these theories and frameworks can be useful to uncover and examine user experiences and technology acceptance, it may not be possible to examine the areas unique to mobile apps and the experiences of this population through this lens. Consistent with other 
studies, my study findings suggest that there are other factors that are overlooked in these theories related to aging, including specific biophysical (e.g. cognitive and physical decline) and psychosocial (e.g. loneliness) characteristics (Chen \& Chan, 2011; Peek et al., 2014), as well as related to living with dementia, such as particular issues experienced by this population (e.g. social isolation, desire for meaningful activities, maintaining longstanding practices, need for safety) (Thordardottir, Fange, Lethin, Gatta, \& Chiatti, 2019). These theories also offer an explanation of areas that impact acceptance of multiple types of technology rather than factors that are more technology specific, such as those unique to mobile apps. For instance, participants spoke about benefits, such as interactivity and integration, which are functions of mobile apps that are distinct from other forms of technology. Moreover, these theories have disregarded the fact that technology acceptance can fluctuate over time as they primarily focussed on the preimplementation stage (when a piece of technology has not been used) instead of the postimplementation stage (when users have used and experienced a technology) (Peek et al., 2014), which is the case in this study. In fact, research has found that perceived ease of use can differ between the pre-implementation and post-implementation stage (Bouwhuis, Meesters, \& Sponselee, 2012, as cited in Peek et al., 2014). Additionally, Peek and colleagues (2014) also found that different types of implementation factors for older adults, such as fear of stigmatization and desire for aging in place, exist which are not considered by prominent technology acceptance theories. Thus, more investigation towards technology acceptance in the form of mobile apps specifically by persons living with dementia and their family caregivers are warranted in order to develop theories suitable for this population. 


\section{Envisioning the Potential of Mobile apps}

Gaps in available mobile apps were identified by participants as they discussed the potential of apps to better support them as family caregivers. In fact, in a study that systematically identified and appraised current apps targeting and available for caregivers of persons living with dementia, Brown and colleagues (2019) found that most apps available have limited features and do not provide the multiple features required to meet the complex needs of caregivers. Supporting those findings, participants in this study indicated two kinds of gaps that currently exist: (1) areas of app functionality; and (2) caregiving needs that apps are addressing.

Specific areas of app functionality that can be improved, such as having multiple language options and multiple functions within a single app, identified by participants finds support in findings from other research. Similarly, in a study by Kerkhof and colleagues (2017) that involved persons living with dementia and their family caregivers, findings indicated the need for the language used in apps to be adjustable in the user's own language. Brown and colleague (2019) found that few apps targeting caregivers were available in languages other than in English. This gap is particularly problematic in a country like Canada as nearly 6.6 million, or 20\%, of Canadians reported speaking a language other than English or French at home (Statistics Canada, 2018).

Participants also wanted apps that are multifunctional, such that the app integrates multiple functions within a single, cohesive program. They explained that this form of a "onestop-shop" in a mobile app would reduce the need to navigate several apps to achieve various goals as they can accomplish it within one app. This was consistent with other studies whereby participants expressed preference towards having a multifunctional app (e.g. Hilliard et al., 2014). In particular, Zelinski and colleagues (2017) found, through their search of all apps 
focused on caregivers of older adults in Google Play and iTunes, that apps mainly addressed specific categories of support, such as information and resources, family communication, and/or caregiver-recipient interactions. They noted that most apps were not comprehensive in nature as only a few had features that addressed more than three categories. As such, the authors recommended that mobile apps provide multiple beneficial support functions to address caregiver's needs in a comprehensive manner. Brown and colleagues (2019) further supported this recommendation as they argued that the more functions an app has, the more likely a caregiver can address their various, complex, and changing caregiving needs within a single app, rather than relying on numerous apps with varying features.

In addition to app functionality, participants also described ideas in addressing caregiving needs that can be materialized into an app. The finding of having an app that enables communication and sharing of information with healthcare providers in this study is consistent with study findings in people living with HIV (Ramanathan, Swendeman, Comulada, Estrin, \& Rotheram-Borus, 2013) and eating disorders (Juarascio, Goldstein, Manasse, Forman, \& Butryn, 2015). The findings of the current study, along with other research, highlight the desire of patients and caregivers to have greater access to their healthcare team. However, Brown and colleagues (2019) found that none of the available apps they reviewed connect caregivers with the care recipient living with dementia's healthcare providers. It is important to note that mobile apps have the ability to collect and organize information and promote true family-centered care by promoting sharing of information and decision-making between healthcare providers and family caregivers (Brown et al., 2019). As such, developing apps to facilitate communication between family caregivers and healthcare providers can address an unmet need in this population. 
A common and priority need that was envisioned were for mobile apps to address safetyrelated issues with the care recipient, such as falls, emergencies, wayfinding, and forgetting to turn off appliances. These issues are prominent among persons living with dementia as they are exposed to higher risk of incidents (e.g. falls, wayfinding) due to gait and balance problems as well as cognitive impairments (Gagnon-Roy et al., 2017). In a study examining the unmet needs in community-dwelling persons living with dementia and their caregivers, Black and colleagues (2013) found that the most common unmet need reported was in the domain of safety, such as fall risk management, home safety evaluation and wander risk management. For family caregivers, managing these issues and balancing risk reduction with encouraging an active lifestyle with their relative living with dementia often presents to be a major challenge and thus, they have been especially receptive to using technology for assistance in this area (Gagnon-Roy et al., 2017). The findings of this study highlight the pressing need of developing apps to address these areas of concern. Supporting this aim, many researchers have started to develop and test apps to assist in home-hazard modification in falls prevention (Ward, Walker-Clarke, \& Holliday, 2017) and to help older adults self-assess and monitor their individual fall risk (Rasche et al., 2017). By supporting persons living with dementia and their family caregivers in these safety-related needs, it can reduce the risk of incidents which can ultimately improve outcomes for the dyad.

\section{Summary}

To summarize, continued use among participants was facilitated by an immediate recognition of the benefits of the mobile app and this was influenced by the characteristics and functions of the apps, including simplicity, easy navigation, and interactivity and integration. Participants also described aspects of apps that required improvement and areas of concerns that 
they wanted mobile apps to address. These findings provide a further understanding of the requirements necessary to meet the needs and preferences of this population. In fact, the findings of this study highlight the need for mobile apps to possess characteristics and functionalities matching the end-users' preferences in order to promote sustained engagement and continued use from the end-users.

\section{Study Implications}

\section{Implications for Education}

The increasing use of mobile apps is facilitating patient- and family-centered care as well as patient engagement in health promotion and maintenance activities (Billings, 2015). Indeed, it is estimated that more than 100,000 health-related apps exist, while more are under development (Xu \& Liu, 2015). The emergence of mobile apps in healthcare will require health and social care professionals to acquire additional skills and competencies, including knowing how to use the technology as well as how to evaluate the quality and appropriateness of a particular app. In fact, the College of Nurses of Ontario (2014a; 2014b) indicated in the entry-to-practice competencies of both Registered Nurses and Registered Practical Nurses that nurses need to understand the implications of informatics and technologies in healthcare as well as to utilize technology in promoting and providing safe nursing care. Similarly, the Canadian Gerontological Nursing Association (2010) noted in the gerontological nursing competencies and standards of practice that gerontological nurses should promote and maintain optimal function, independence and safety among older adults through the use of appropriate technology and assistive devices. Thus, educators have a significant role in updating curricula and programs to equip students in training to be health and social care professionals with those skills and competencies (Billings, 2015). The National League for Nursing (NLN; 2015) recommended that educators integrate the 
use of mobile technology into learning experiences and clinical experiences. Furthermore, they asserted that faculty must align curricula with current technological advances and incorporate competencies in informatics and technology through the program of learning (NLN, 2015). It is imperative to consider these recommendations in order to prepare students for the technological world of health care, especially considering the growing use of mobile apps.

\section{Implications for Practice}

The present findings have implications for practice regarding dementia care and family caregiving. Participants in this study were most likely to have received information about various kinds of mobile apps from doing searches on their own or less frequently, from family and friends. This is in line with other research where family caregivers of persons living with dementia reported that rely on their own personal knowledge rather than health and social care professionals when it comes to using technology in caregiving (Gibson et al., 2015; Newton et al., 2016). They stressed that minimal information and support from health and social care professionals about how to access pieces of technology and how it can be used to assist in their caregiving role is provided to them (Gibson et al., 2015; Newton et al., 2016). Thus, health and social care professionals who frequently meet with persons living with dementia and their family members should have up-to-date knowledge of available and credible mobile apps for use in dementia care and caregiving. They should also be aware of the capabilities and limitations of the apps and provide appropriate support and education. Persons living with dementia and their

family caregivers should be offered possible mobile app resources throughout the progression of the condition as their needs may change or expand. This can be accomplished through regular information and training for health and social care professionals, and including information about mobile apps and mHealth in healthcare education. 


\section{Implications for Future App Development}

The study findings underscore the need for developers and researchers to involve endusers (e.g. persons living with dementia and their family caregivers) throughout the app development process. The value of incorporating end-user perspectives during the early stages of design and development of new mobile apps is well supported by a growing body of research (Hirchey et al., 2018; Meiland et al., 2017; Reynoldson et al., 2014). Researchers argue that the potential of mobile app interventions can not be materialized unless the design and development take in account of the users' experiences (Hilliard et al., 2014; Reynoldson et al., 2014). The findings unveiled the complex and nuanced caregiving experiences of family caregivers and thus, it is essential to holistically examine the context in which family caregivers and their care recipients use mobile apps in order to develop appropriate suitable apps.

The findings also suggest that the usefulness of an app often changes based on the family caregivers' needs or the condition of the care recipient. Hence, designers will need to consider and adapt the mobile app to the changing needs of its end-users in order for it to continue to be perceived as useful over a long period. In other words, it is important to evaluate whether an app can continue to provide support throughout each stage of the care recipient's condition as needs may evolve as the condition progresses and/or as changes arise in the life of the family caregiver. Overall, aligning with the current literature, the study findings suggest that the functionalities and features of apps should match the individual needs, wishes and abilities of persons living with dementia and their family caregivers.

\section{Implications for Policy}

An increasingly number of care recipients and their family caregivers are using mobile apps in their lives. However, participants in this study identified areas of caregiving needs that 
they wish mobile apps could address, which is presently not available. Thus, government bodies and health care organizations should consider providing more funding towards projects aimed at developing apps for this population and their needs. It is important to note that some organizations, although scarce, have already funded and developed mobile apps for persons living with dementia and their family caregivers with promising outcomes. They include the Dementia Advisor app funded in part by the Government of Canada's Social Development Partnership Program as well as the Dementia Talk app funded in part by the Centre for Aging and Brain Health Innovation at Baycrest and the Government of Canada's New Horizons for Seniors Program - which were both developed by Sinai Health System and the Reitman Centre in Toronto, Canada. However, both apps are only addressing one important caregiving need as they were designed to support caregivers in tracking and responding to changing behaviours and functions of persons living with dementia. As shown in the findings, family caregivers experience a variety of complex challenges and needs in their caregiving role and thus, more apps need to be developed to address those needs. Lastly, legislators should also consider creating policies that will support research and development towards technological solutions that can address the needs of this population.

\section{Implications for Research}

The demographic profile of the participants in this study warrant additional studies to explore the experiences of different groups of family caregivers. For instance, all the participants in the study were female and between the ages of 18 and 35 years old and thus, future studies should explore the experiences of family caregivers who are male as well as family caregivers who are younger than 18 years old and above 35 years old. This is important to consider as researchers have found that the family caregiving experiences for male caregivers differ from 
those of female caregivers (Mc Donnell \& Ryan, 2011). Researchers have also found that when compared to their younger counterparts, people older than 30 years old have different knowledge and attitudes towards the use of mobile apps (Rahman et al., 2017). Additionally, given that none of the participants were living in a rural setting, further research can explore experiences of family caregivers in rural communities in consideration of geographic disparities and barriers to technology use that could exist between rural and non-rural populations (Ruggiano, Brown, Li, \& Scaccianoce, 2018). Studies could also examine the experiences of using mobile apps among other types of caregivers to assist with care, such as spouses and friends.

Moreover, since this study only focused on the experiences of adult child family caregivers, further research should explore the experiences of the care recipient using mobile apps. The findings indicated that persons living with dementia, with the support of the family caregivers, are using mobile apps in their daily lives. To develop technologies that are useful and valuable for persons living with dementia, a thorough understanding of the different types and stages of dementia and its associated experiences are required as persons living with dementia should not be treated as a homogenous group given that experiences will be different for people with various stages or types of dementia (Meiland et al., 2017; Suijkerbuijk et al., 2019). Thus, future studies should involve persons living with dementia to explore their experiences of living with dementia, their needs, and their perspectives as researchers have found that persons living with dementia can express their needs and preferences consistently, even in an advanced stage of dementia (Meiland et al., 2017; Span et al., 2013). Researchers have also indicated that their participation in research have improved the usefulness and acceptability of the various technological devices developed (Span, Hettinga, Vernooij-Dassen, Eefsting, \& Smits, 2013). Persons living with dementia's extensive insight into their dynamic needs, wishes and abilities 
can also inform health and social care professionals on what types of mobile apps are meaningful, appropriate and suitable for this population during care provision. Ultimately, in line with person-centered care, future studies should provide persons living with dementia their desired level of involvement in the development of mobile apps as well as design research methods that are inclusive and accessible for this population. To facilitate involvement from this population, methods employed in the literature and recommended by researchers include interviews, observations, focus groups, workshops, and questionnaires (Suijkerbuijk et al., 2019; Span et al., 2013).

This study also revealed several new findings that offer direction for future research in this area. For example, investigating the impact of apps in facilitating meaningful activities on the care recipient's well-being. Additionally, research evaluating features and functions of mobile apps that promote usability and continued use among this population would be beneficial when designing future apps targeted specifically for persons living with dementia. Longitudinal studies can also be implemented to examine the use, benefits, and barriers of mobile apps over time. These studies can provide information on when is the most appropriate time to introduce mobile apps and for what areas as well as what type of apps are most helpful during the various stages of dementia. Future studies can also involve both the family caregiver and care recipient as a dyad in order to further explore the dynamics of dyadic interaction and their effects on both parties when mobile apps are used in caregiving. Overall, additional research is necessary to assess the impact of app use on the outcomes for the care recipient and their family caregivers.

There is also a dearth of research on health and social care professionals' perceptions of apps (Peng et al., 2016). This is problematic as persons living with dementia and their family caregivers require reliable, accurate and up-to-date information in order to access mobile apps 
for assistance and they often rely on health and social care professionals for guidance (Gibson et al., 2016). As such, future research regarding their awareness about and experience with mobile apps is warranted.

\section{PART II: PARTICIPANTS' EXPERIENCE WITH PHOTO-ELICITATION INTERVIEWING (PEI)}

The use of photographs in studies is prevalent in the literature, and in the field of dementia research (e.g. Evans, Robertson, \& Candy, 2016; Genoe \& Dupuis, 2013; Wiersma, 2011). Yet, very little of the previous research have specifically explored the experiences of using photographs in interviews from the perspective of participants (see, for instance, Edmondson, Brennan \& House, 2018; Warner, Johnson, \& Andrews, 2016). Unlike a majority of studies, the study findings focused on the participants' own perspectives, rather than those of the researchers, of engaging in PEI.

Participants found that the photography activity was flexible enough to fit within their everyday schedule and was suitable for the range of differing abilities they possessed. For example, Cassandra opted to use a professional camera and computer software to edit her photographs, while others took the photographs through their smartphones. All participants were able to produce five photographs that they were willing to share and discuss. While at first, participants were concerned about the difficulty of transforming abstract ideas into photographs, the overall consensus was that it was an enjoyable and worthwhile activity. Using a visual methodology that focused on mobile apps and how they are used in their caregiving role, participants were enabled to portray their experiences in a comprehensive manner. Specifically, by using the autodriving approach whereby the photographs were taken by participants, participants in this study were able to choose the information they wanted to share and how they 
wanted to represent their experiences (Shell, 2014). Importantly, this process enabled participants to control the process of image production, dissemination, and narration (Asiedu, Carroll, Griffin, Hurt, \& Mundi, 2018).

As with other research (Shell, 2014), the findings unveiled that the photographs and the ensuing dialogue provided participants the opportunity to fully explore and reflect more in-depth on their experiences. Fascinatingly, the photographs and the subsequent dialogue also encouraged participants to talk about things they viewed as normal parts of everyday life which often goes unnoticed (Marsh, Shawe, Robinson, \& Leamon, 2016). This was particularly salient when participants, through our discussion together, came to realize aspects of their caregiving experiences and their use of mobile apps that were overlooked. Additionally, the reflexive effect of using photographs in interviews also led to the discussion of thoughts and feelings that have laid dormant (Marsh et al., 2016). Using photographs to represent caregiving experiences required effort, abstract thinking, and reflexivity from participants (Edmondson et al., 2018). Taking photographs gave participants a new perspective of their experiences and also a greater appreciation for the role of mobile apps in their caregiving role. Some of photographs portrayed a variety of uses of mobile apps and conveyed a sense of the participant's innovative, organized and well-thought-out strategies that constituted their caregiving practices. Overall, participants felt that the photographs encouraged them to talk about aspects of their experiences that may not have been discussed in a verbal-only interview.

The photographs also promoted richer dialogue and introduced new dimensions of the participants' experiences that I, as the researcher, had not considered. Furthermore, the photographs helped build rapport between the participant and myself by bridging the distance between the researcher and participant. This can be seen in the depth and extent of the topics that 
were discussed in the second interview which was facilitated by the use of photographs. The photographs gave enlightening and personal re-interpretations of known phenomena. For example, grief and loss is an accepted impact of dementia (Lindauer \& Harvath, 2014) but seeing an image of blurred Polaroids and hearing Cassandra describe how this represents her reflection of the effect of dementia on her grandmother gave a much more vivid understanding of its impact. Arguably, the photographs provided insight into difficult, emotional or otherwise sensitive issues and experiences (Clark \& Morriss, 2017). Such issues, including grief and loss, decline in health of a loved one, and management of changes in life associated with dementia as discussed by many of the participants, can be difficult to articulate and through the use of photography, participants felt it made describing challenging topics easier. In cases such as these, Clark and Morriss (2017) explained that photographs are beneficial because they can be viewed as a neutral or displaced element around which to express and advance discussion. In other words, they act "as a kind of 'third object' around which participants and researchers can focus" (Clark \& Morriss, 2017, p. 36), making sensitive topics less difficult to discuss and articulate.

The experiences of the participants who engaged in the use of photographs in the followup interview align with findings from other studies that have explored participants' experiences with PEI (e.g. Edmondson et al., 2018; Warner et al., 2016). Also consistent with other studies, I found that participant-produced photographs with subsequent interviews to be a useful approach for generating rich data. Using participant-produced photographs in PEI enabled participants to have control over image creation and meaning-making. As a result, this yielded a more in-depth discussion of participants' experiences and elicited longer and more comprehensive follow-up interviews. Including photographs facilitated richer dialogue and offered greater insight than could be accomplished through verbal-only interviews when I compared the depth of the 
discussion in the first interview versus the second in this study. Meo (2010) also argued that compared to verbal-only interviews, interviews that involved photographs allowed for the emergence of unexpected topics, enabled making sense of some data which otherwise would have been challenging to interpret, and elicited longer and more enjoyable discussions.

Ultimately, given that the photographs are generated by the participants themselves, the process led to a more comprehensive exploration and discussion of the experiences that are most salient to participants. As a result, the photographs and subsequent dialogues offered a glimpse to the realities of the lives of participants that was not previously explored (Wong, George, Godfrey, Le, \& Pereira, 2019).

\section{Implications for Research}

While a wealth of literature attests to the benefits of PEI, researchers need to carefully evaluate whether this technique would help them gain a deeper exploration of the phenomenon under study. They will also need to assess the feasibility of carrying out PEI given that the time and effort involved is more demanding than traditional, verbal-only interviews (Meo, 2010). As demonstrated in Chapter 4, researchers will have to manage the different types of ethical, methodological and practical challenges that can emerge before, during and after photoelicitation interviews (Meo, 2010). Nevertheless, drawing on my own positive experiences, PEI can ultimately lead to a richer understanding of the phenomenon under study. Past research endeavours have also demonstrated that including photographs in the research process as suitable for use with many different populations, ranging from children (Torre \& Murphy, 2015) to persons living with dementia (Genoe \& Dupuis, 2013), and different lived experiences, such as living with depression (Sandhu, Ives, Birchwood, \& Upthegrove, 2013) and cancer (Wong et al., 2019). Riley and Manias (2004) emphasized that the use of photography needs to be promoted in 
nursing as a method that can enrich traditional forms of data collection. Moreover, Balmer and colleagues (2015) highlighted that nurse researchers are able to access patients' social environments and lived experiences in a way that may be richer than interviewing alone. They also contended that this will result in a greater understanding of the impact of illness. By presenting the study data in the form of photographs, the authors further elaborated that it can offer a powerful way of communicating the experiences of participants to various professional and lay groups, and has the potential to reach a far greater and more diverse audience. Based on my experiences and those of other researchers, PEI has the potential to not only empower participants to become more engaged in the research process, but also enable researchers to obtain more rich, holistic and multifaceted data.

\section{Strengths and Limitations}

To the best of my knowledge, this is the first study that explored the experiences of family caregivers of persons living with dementia who use mobile apps in their caregiving role. Thus, the findings contribute to a growing body of evidence about the potential benefits of mobile apps for family caregivers as well as the importance of considering the needs of the persons living with dementia and their family caregivers during all stages of mobile app development. Furthermore, in this study, the use of photograph added a supplementary layer of explanation to participants' narratives and experiences. In doing so, this enabled me to have a richer understanding and to make more comprehensive interpretations of the participants' experiences. Sandhu and colleagues (2013) emphasized that PEI has a unique focus on involving and engaging participants in the process of data collection (taking photographs) and initial analysis (explaining what the photographs mean during the interview) which adds to the credibility of the data. The authors argued that it allows for participant's own views about the 
meaning and importance of various aspects of the data to be highlighted. As such, in this study, I was able to collect ample data using multiple methods and data collection points which ultimately enhanced the richness of the findings.

Several limitations to this study should be considered. Participants were all female and between the age of 18 to 35 years old which does not fully represent the population of adult child family caregivers of persons living with dementia. Confirming the trends in literature, there is a paucity of studies pertaining to male caregiving in the dementia context (Mc Donnell \& Ryan, 2011); perhaps due to barriers to participation in research that exist for men or gendered burden of care as family caregivers are more likely to be women in Canada (Turcotte, 2013). Moreover, adult child family caregivers not within the ages of 18 to 35 years old did not participate in this study and thus, the experiences and perspectives of this particular group of family caregivers who belong to different generations than the participants in this study are missing from the findings.

Given the purpose of this study, only family caregivers were recruited rather than both the care recipient and the family caregiver together as a dyad. As such, the findings may be limited in providing a comprehensive understanding of the shared changes and experiences that occur as dementia progresses in the dyad, including their relationship, reciprocal interaction, and level of interdependence (Moon \& Adams, 2012). Persons living with dementia also did not participate in this study and while family caregivers can provide important insight into the care recipient's experiences, it cannot act as a substitution as the care recipient may have different needs and perspectives regarding mobile apps.

In addition, the study was conducted in Ontario with the majority of participants from Toronto, thus the contexts of their caregiving experiences may not be the same as those from 
other geographical regions. Lastly, although participants were offered the option to have the interviews in-person, all of them opted to have telephone interviews. With telephone interviews, I might have missed important visual cues and nonverbal gestures. This was an unavoidable limitation given that it was the participants' decision. Considering that all of the participants were either working or in school full-time in addition to caregiving, it could be plausible that participants felt telephone interviews were more convenient to fit into their busy schedules. Alternatively, researchers have argued that participants may have felt more comfortable and relaxed in sharing information in telephone interviews than in-person (Novick, 2008).

\section{Conclusion}

What are the user experiences of family caregivers using mobile apps to assist in caregiving for their loved ones living with dementia in the community?

Pursuing this line of inquiry, I gained a richer understanding of the myriad and complex everyday challenges that family caregivers face and the impact mobile apps have on their caregiving role. I began this thesis research with my own story as it related to my experiences as a care recipient, family caregiver, and formal healthcare provider. I explicated how this experience informed the direction of my thesis. As I began my thesis journey, through reviewing the literature, I came to understand that no research has explored the experiences of family caregivers of persons living with dementia in the context of mobile apps and how as a result, limited mobile apps are developed to address this population's needs.

With the exploratory approach used in this study, user experiences of family caregivers of persons living with dementia were discovered which aptly illustrated the multidimensional nature of family caregiving. This study adds to our growing understanding of the complexity of family caregiving to persons living with dementia and how mobile apps can address the diverse 
needs and issues that the care recipient and their family caregivers may face on a regular basis. Additionally, this study demonstrated how technology can mitigate disruptions associated with dementia on the individual and the family as a whole through the novel use of mobile apps. These findings illuminated that apps not only helped with care provision, but also allowed for richer interpersonal connections to form as it enabled for relationships to flourish and in some cases, served as a platform for reciprocal caring. The findings also broadened the concept of self extension (Belk, 1988) as it revealed that mobile apps can serve as an extension of the caring self. Through this study, the narratives of the participants highlighted the need to understand their experiences as their preferences, perspectives, and most importantly, their creative solutions to everyday challenges with which they are intimately familiar with are imperative to future mobile app developments. As a result, this study builds on existing models of person-centered care to recognize the significance of technologies for family caregivers in supporting the social world of care recipients. This study further expands our understanding that person-centred care can extend to include tailored use of technology such as mobile apps. Such understanding, while preliminary, provides some beginning direction in this regard for those who are involved in dementia care, including family caregivers, persons living with dementia, and health and social care professionals. While this study has focused on family caregivers of persons living with dementia residing at home, findings can potentially inform practice in both community and facility care settings. 


\section{EPILOGUE}

FINAL THOUGHTS 
As I reflected upon the experiences in my thesis journey, I realized that the narratives of participants which showcased their resilience and fortitude during some of the most difficult and challenging times will have a lasting impact in my life. Thus, I wanted to end my thesis simply with the following photograph and quote:

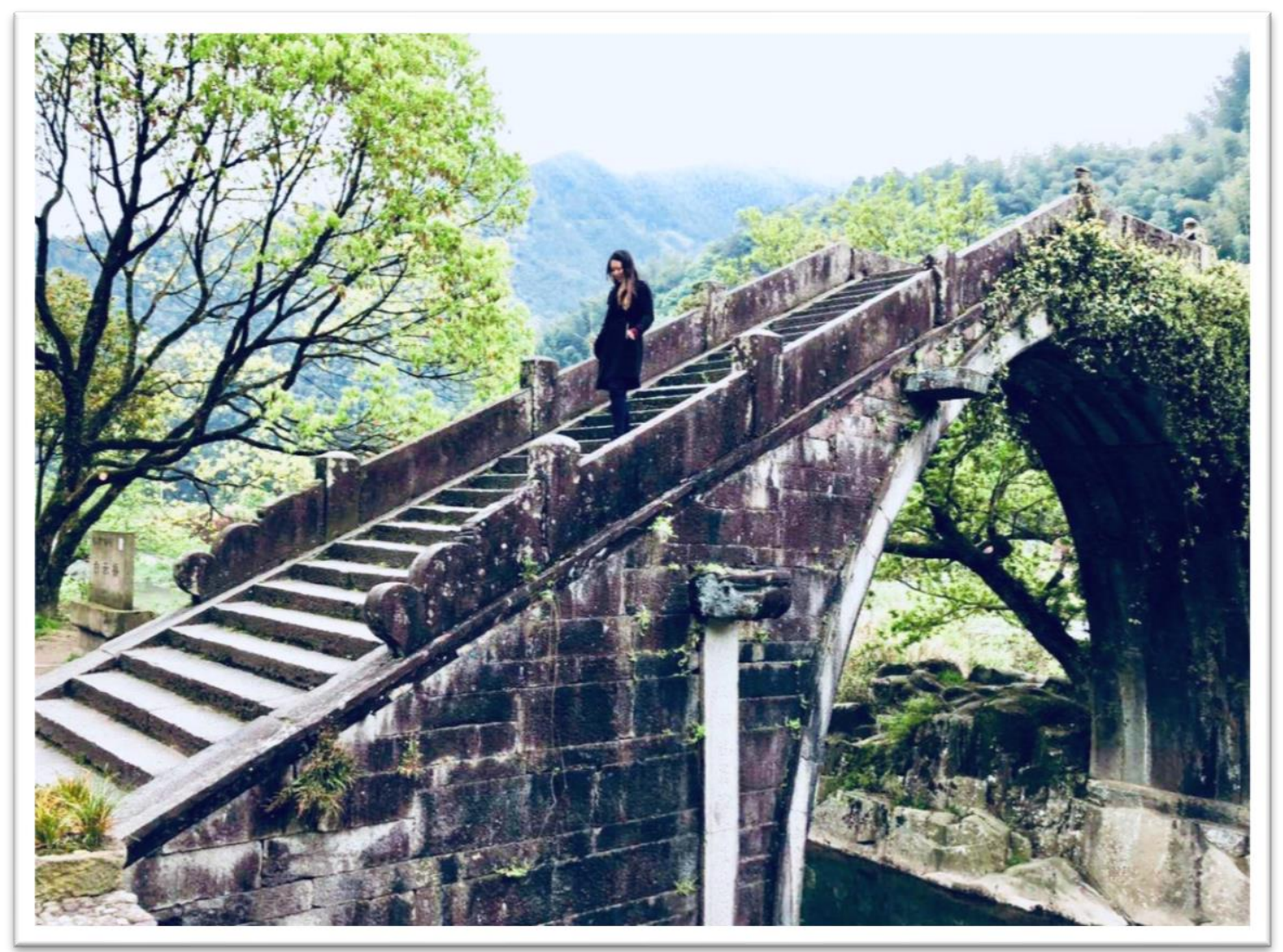

A photograph of me taken by my father in his hometown in China.

"A bridge can still be built, while the bitter waters are flowing beneath."

- Anthony Liccione

(American Poet and Author) 


\section{APPENDIX A}

Mobile Apps in the Dementia Context

\begin{tabular}{|c|c|c|c|c|c|c|c|}
\hline \# & $\begin{array}{l}\text { Authors } \\
\text { \& Year }\end{array}$ & Name of App & $\begin{array}{l}\text { Description of } \\
\text { App }\end{array}$ & $\begin{array}{l}\text { Targeted } \\
\text { Group }\end{array}$ & Design/Methods & $\begin{array}{l}\text { Domain } \\
\text { of App }\end{array}$ & Significant Findings \\
\hline 1 & $\begin{array}{l}\text { Abu- } \\
\text { Dalbouh } \\
\text { et al., } \\
2015\end{array}$ & $\begin{array}{l}\text { A Mobile } \\
\text { Reminder } \\
\text { App for } \\
\text { Elderly and } \\
\text { Alzheimer's } \\
\text { patients } \\
\text { (prototype) }\end{array}$ & $\begin{array}{l}\text { To remind } \\
\text { individuals who are } \\
\text { elderly and living } \\
\text { with Alzheimer's } \\
\text { disease the dates } \\
\text { and dose of their } \\
\text { medications, food } \\
\text { times, and } \\
\text { important events. }\end{array}$ & $\begin{array}{l}\text { Individuals } \\
\text { who are } \\
\text { elderly and } \\
\text { living with } \\
\text { Alzheimer's } \\
\text { disease }\end{array}$ & $\begin{array}{l}\text { Designed by authors based } \\
\text { on the literature. }\end{array}$ & $\begin{array}{l}\text { Manage- } \\
\text { ment } \\
\text { Care } \\
\text { provision } \\
\text { Quality } \\
\text { of life }\end{array}$ & $\begin{array}{l}\text { Has potential to assist } \\
\text { individuals who are } \\
\text { elderly and living with } \\
\text { Alzheimer's disease live } \\
\text { independently and } \\
\text { improve their quality of } \\
\text { life. }\end{array}$ \\
\hline 2 & $\begin{array}{l}\text { Acharya } \\
\text { et al., } \\
2017\end{array}$ & $\begin{array}{l}\text { Dementia } \\
\text { HelpDesk }\end{array}$ & $\begin{array}{l}\text { To provide } \\
\text { practical support } \\
\text { for family } \\
\text { caregivers of a } \\
\text { loved one with } \\
\text { dementia, including } \\
\text { GPS navigation, } \\
\text { fall detection, mind } \\
\text { games, and a } \\
\text { doctor registry. }\end{array}$ & $\begin{array}{l}\text { Persons } \\
\text { living with } \\
\text { dementia } \\
\text { and their } \\
\text { family } \\
\text { caregivers }\end{array}$ & Not mentioned. & $\begin{array}{l}\text { Manage- } \\
\text { ment } \\
\text { Care } \\
\text { provision } \\
\text { Quality } \\
\text { of life }\end{array}$ & $\begin{array}{l}\text { In the preliminary stages. } \\
\text { Authors hope to involve } \\
\text { healthcare professionals in } \\
\text { future stages. }\end{array}$ \\
\hline 3 & $\begin{array}{l}\text { Bhatta- } \\
\text { charyya et } \\
\text { al., } 2017\end{array}$ & $\begin{array}{l}\text { Living and } \\
\text { Dying Well }\end{array}$ & $\begin{array}{l}\text { To provide an } \\
\text { accessible } \\
\text { educational } \\
\text { resource to }\end{array}$ & $\begin{array}{l}\text { Persons } \\
\text { living with } \\
\text { dementia }\end{array}$ & $\begin{array}{l}\text { Developed with a wide } \\
\text { range of healthcare } \\
\text { professionals and } \\
\text { educationalists in }\end{array}$ & $\begin{array}{l}\text { Manage- } \\
\text { ment }\end{array}$ & $\begin{array}{l}\text { Preliminary evaluation } \\
\text { from caregivers indicate } \\
\text { that the app can enhance } \\
\text { family preparedness of }\end{array}$ \\
\hline
\end{tabular}




\begin{tabular}{|c|c|c|c|c|c|c|c|}
\hline \# & $\begin{array}{l}\text { Authors } \\
\text { \& Year }\end{array}$ & Name of App & $\begin{array}{c}\text { Description of } \\
\text { App }\end{array}$ & $\begin{array}{l}\text { Targeted } \\
\text { Group }\end{array}$ & Design/Methods & $\begin{array}{l}\text { Domain } \\
\text { of App }\end{array}$ & Significant Findings \\
\hline & & $\begin{array}{l}\text { with } \\
\text { Dementia }\end{array}$ & $\begin{array}{l}\text { promote } \\
\text { understanding of } \\
\text { dementia, its } \\
\text { impact on those } \\
\text { affected, and the } \\
\text { key issues in end of } \\
\text { life care. }\end{array}$ & $\begin{array}{l}\text { and their } \\
\text { caregivers }\end{array}$ & $\begin{array}{l}\text { collaboration with the } \\
\text { Alzheimer's Society, the } \\
\text { Gold Standards Framework } \\
\text { and the End of Life } \\
\text { Partnership (EOLP). }\end{array}$ & $\begin{array}{l}\text { Care } \\
\text { provision } \\
\text { Quality } \\
\text { of life }\end{array}$ & $\begin{array}{l}\text { informal/family caregivers } \\
\text { as well as can be for } \\
\text { formal healthcare } \\
\text { providers in care home } \\
\text { settings. }\end{array}$ \\
\hline 4 & $\begin{array}{l}\text { Brown et } \\
\text { al., } 2016\end{array}$ & CareHeroes & $\begin{array}{l}\text { To support } \\
\text { individuals caring } \\
\text { for loved ones with } \\
\text { Alzheimer's } \\
\text { disease or other } \\
\text { forms of dementia. }\end{array}$ & $\begin{array}{l}\text { Family } \\
\text { caregivers, } \\
\text { case } \\
\text { managers, } \\
\text { and primary } \\
\text { care } \\
\text { providers }\end{array}$ & $\begin{array}{l}\text { Informed by the Family- } \\
\text { Centered Theory. } \\
\text { Employed a user-centered } \\
\text { design and involved end- } \\
\text { users in the content } \\
\text { development. }\end{array}$ & $\begin{array}{l}\text { Manage- } \\
\text { ment } \\
\text { Care } \\
\text { provision } \\
\text { Quality } \\
\text { of life }\end{array}$ & $\begin{array}{l}\text { Caregivers used and } \\
\text { valued the capacity to } \\
\text { assess and document care } \\
\text { recipient behaviour } \\
\text { disturbances and mood on } \\
\text { a regular basis through an } \\
\text { app. The resulting } \\
\text { information are helpful to } \\
\text { healthcare providers } \\
\text { working with caregivers to } \\
\text { manage care recipient } \\
\text { behaviours and caregiver } \\
\text { distress. }\end{array}$ \\
\hline 5 & $\begin{array}{l}\text { Burns et } \\
\text { al., } 2017\end{array}$ & BPSD Guide & $\begin{array}{l}\text { To support } \\
\text { clinicians by } \\
\text { providing ready } \\
\text { access to concise, } \\
\text { evidence-based } \\
\text { information } \\
\text { relevant to the ten }\end{array}$ & $\begin{array}{l}\text { Formal } \\
\text { caregivers }\end{array}$ & $\begin{array}{l}\text { Content incorporates } \\
\text { evidence-based outcomes } \\
\text { from an extensive literature } \\
\text { review and consultation } \\
\text { with experienced clinicians } \\
\text { across Australia. }\end{array}$ & $\begin{array}{l}\text { Manage- } \\
\text { ment } \\
\text { Care } \\
\text { provision } \\
\text { Quality } \\
\text { of life }\end{array}$ & $\begin{array}{l}\text { Clinicians reported that the } \\
\text { app was useful in } \\
\text { supporting them to care for } \\
\text { persons living with } \\
\text { dementia presenting with } \\
\text { BPSD by providing them }\end{array}$ \\
\hline
\end{tabular}




\begin{tabular}{|c|c|c|c|c|c|c|c|}
\hline$\#$ & $\begin{array}{l}\text { Authors } \\
\text { \& Year }\end{array}$ & Name of App & $\begin{array}{c}\text { Description of } \\
\text { App }\end{array}$ & $\begin{array}{l}\text { Targeted } \\
\text { Group }\end{array}$ & Design/Methods & $\begin{array}{l}\text { Domain } \\
\text { of App }\end{array}$ & Significant Findings \\
\hline & & & $\begin{array}{l}\text { most commonly } \\
\text { presenting } \\
\text { behavioural and } \\
\text { psychological } \\
\text { symptoms of } \\
\text { dementia (BPSD). }\end{array}$ & & & & $\begin{array}{l}\text { ready access to evidence- } \\
\text { based information. }\end{array}$ \\
\hline 6 & $\begin{array}{l}\text { Cook \& } \\
\text { Twidle, } \\
2016\end{array}$ & $\begin{array}{l}\text { Serious } \\
\text { Games }\end{array}$ & $\begin{array}{l}\text { To increase } \\
\text { awareness of } \\
\text { symptoms of } \\
\text { dementia - } \\
\text { specifically } \\
\text { Alzheimer's } \\
\text { Disease. }\end{array}$ & $\begin{array}{l}\text { General } \\
\text { Public }\end{array}$ & $\begin{array}{l}\text { Employed a user-centric } \\
\text { design and lean user } \\
\text { experience methodology to } \\
\text { develop the prototypes. } \\
\text { Evaluation of the app was } \\
\text { undertaken with three stages } \\
\text { and } 15 \text { end-users. }\end{array}$ & $\begin{array}{l}\text { Aware- } \\
\text { ness }\end{array}$ & $\begin{array}{l}\text { Shown to increase } \\
\text { knowledge of symptoms } \\
\text { and behaviours relating to } \\
\text { Alzheimer's disease } \\
\text { among users during } \\
\text { evaluation. }\end{array}$ \\
\hline 7 & $\begin{array}{l}\text { Davis et } \\
\text { al., } 2014\end{array}$ & Story-Call & $\begin{array}{l}\text { To deliver } \\
\text { personalized story- } \\
\text { telling support, } \\
\text { dementia care } \\
\text { information, and } \\
\text { community } \\
\text { resources to } \\
\text { caregivers of } \\
\text { community- } \\
\text { dwelling persons } \\
\text { living with } \\
\text { dementia. }\end{array}$ & $\begin{array}{l}\text { Informal } \\
\text { caregivers }\end{array}$ & $\begin{array}{l}\text { Grounded in the Resiliency } \\
\text { Model of Family Stress, } \\
\text { Adjustment and Adaptation. } \\
\text { Developed by a team of } \\
\text { experts in mobile } \\
\text { technology and dementia } \\
\text { care (e.g. nurse, software } \\
\text { specialist, gerontologist, } \\
\text { linguist). }\end{array}$ & $\begin{array}{l}\text { Manage- } \\
\text { ment } \\
\text { Care } \\
\text { provision } \\
\text { Quality } \\
\text { of life }\end{array}$ & $\begin{array}{l}\text { Family caregivers who } \\
\text { evaluated the app felt that } \\
\text { continuing to use it would } \\
\text { enable them to handle } \\
\text { stress and family } \\
\text { relationships more } \\
\text { effectively, as well as find } \\
\text { community healthcare } \\
\text { resources more easily. }\end{array}$ \\
\hline
\end{tabular}




\begin{tabular}{|c|c|c|c|c|c|c|c|}
\hline \# & $\begin{array}{l}\text { Authors } \\
\text { \& Year }\end{array}$ & Name of App & $\begin{array}{c}\text { Description of } \\
\text { App }\end{array}$ & $\begin{array}{c}\text { Targeted } \\
\text { Group }\end{array}$ & Design/Methods & $\begin{array}{l}\text { Domain } \\
\text { of App }\end{array}$ & Significant Findings \\
\hline 8 & $\begin{array}{l}\text { Docking } \\
\text { et al., } \\
2018\end{array}$ & $\begin{array}{l}\text { The Pain } \\
\text { Assessment } \\
\text { App }\end{array}$ & $\begin{array}{l}\text { To help formal } \\
\text { caregivers (health } \\
\text { professionals) } \\
\text { assess pain in } \\
\text { patients living with } \\
\text { advanced dementia. }\end{array}$ & $\begin{array}{l}\text { Formal } \\
\text { caregivers }\end{array}$ & $\begin{array}{l}\text { Developed in collaboration } \\
\text { with the Computing and } \\
\text { Mathematical Sciences } \\
\text { department at the University } \\
\text { of Greenwich. } \\
\text { Designed based on the } \\
\text { Abbey Pain Scale. } \\
\text { Conducted usability testing } \\
\text { with paramedic students and } \\
\text { a Delphi panel of qualified } \\
\text { paramedics. Usability } \\
\text { testing focus groups were } \\
\text { conducted. }\end{array}$ & $\begin{array}{l}\text { Manage- } \\
\text { ment } \\
\text { Care } \\
\text { provision }\end{array}$ & $\begin{array}{l}\text { The results showed that } \\
\text { the app was a potentially } \\
\text { useful tool in the } \\
\text { prehospital setting. } \\
\text { "By providing access to a } \\
\text { tool specifically developed } \\
\text { to help identify/assess pain } \\
\text { in a user-friendly format, } \\
\text { paramedics are likely to } \\
\text { have increased knowledge } \\
\text { and confidence in } \\
\text { assessing pain in patients } \\
\text { with dementia" (p. 1121). }\end{array}$ \\
\hline 9 & $\begin{array}{l}\text { Filoteo et } \\
\text { al., } 2018\end{array}$ & Remin $X$ & $\begin{array}{l}\text { To provide a } \\
\text { platform for users } \\
\text { to upload pictures } \\
\text { and narrations to } \\
\text { create slideshow } \\
\text { stories depicting } \\
\text { important moments } \\
\text { in the person living } \\
\text { with dementia's } \\
\text { life. }\end{array}$ & $\begin{array}{l}\text { Informal } \\
\text { caregivers }\end{array}$ & $\begin{array}{l}\text { Developed by Dthera } \\
\text { Sciences and based on the } \\
\text { concept of reminiscence } \\
\text { therapy. } \\
\text { Involved } 14 \text { persons living } \\
\text { with mild to moderate } \\
\text { dementia and their } \\
\text { caregivers in the evaluation } \\
\text { of the app using various } \\
\text { questionnaires. }\end{array}$ & $\begin{array}{l}\text { Quality } \\
\text { of life }\end{array}$ & $\begin{array}{l}\text { Persons living with } \\
\text { dementia reported } \\
\text { significantly less anxiety, } \\
\text { depression, and overall } \\
\text { emotional distress after } \\
\text { having viewed their story } \\
\text { via the app. } \\
\text { Caregivers also reported } \\
\text { that the care recipient } \\
\text { appeared less emotionally } \\
\text { distressed. }\end{array}$ \\
\hline
\end{tabular}




\begin{tabular}{|c|c|c|c|c|c|c|c|}
\hline$\#$ & $\begin{array}{l}\text { Authors } \\
\text { \& Year }\end{array}$ & Name of App & $\begin{array}{c}\text { Description of } \\
\text { App }\end{array}$ & $\begin{array}{c}\text { Targeted } \\
\text { Group }\end{array}$ & Design/Methods & $\begin{array}{c}\text { Domain } \\
\text { of App }\end{array}$ & Significant Findings \\
\hline 10 & $\begin{array}{l}\text { Halbach } \\
\text { et al., } \\
2018\end{array}$ & mYouTime & $\begin{array}{l}\text { To provide help on } \\
\text { demand and covers } \\
\text { topics on medical } \\
\text { and psychological } \\
\text { issues; } \\
\text { communication, } \\
\text { legal and financial } \\
\text { issues; and } \\
\text { practical advice for } \\
\text { everyday } \\
\text { challenges. }\end{array}$ & $\begin{array}{l}\text { Informal } \\
\text { caregivers }\end{array}$ & $\begin{array}{l}\text { The learning units were } \\
\text { developed by the academic } \\
\text { community at } \\
\text { Diakonhjemmet and } \\
\text { Western Norway University } \\
\text { of Applied Sciences. } \\
\text { The authors also surveyed } \\
\text { available literature and } \\
\text { research as well as } \\
\text { complemented findings with } \\
\text { data from focus group } \\
\text { interviews with informal } \\
\text { caregivers. }\end{array}$ & $\begin{array}{l}\text { Manage- } \\
\text { ment } \\
\text { Care } \\
\text { provision } \\
\text { Quality } \\
\text { of life }\end{array}$ & $\begin{array}{l}\text { The authors reported a } \\
\text { high degree of user } \\
\text { satisfaction and a high } \\
\text { rating of experienced } \\
\text { usefulness of the app } \\
\text { among users. }\end{array}$ \\
\hline 11 & $\begin{array}{l}\text { Imbeault } \\
\text { et al., } \\
2018\end{array}$ & AP@LZ & $\begin{array}{l}\text { To support the day- } \\
\text { to-day activities of } \\
\text { persons living with } \\
\text { Alzheimer's } \\
\text { disease. }\end{array}$ & $\begin{array}{l}\text { Persons } \\
\text { living with } \\
\text { Alzheimer's } \\
\text { disease }\end{array}$ & $\begin{array}{l}\text { Created by the authors, } \\
\text { which is made up of a } \\
\text { multidisciplinary team. } \\
\text { Five persons living with } \\
\text { Alzheimer's disease were } \\
\text { recruited to test the app. }\end{array}$ & $\begin{array}{l}\text { Manage- } \\
\text { ment } \\
\text { Care } \\
\text { provision } \\
\text { Quality } \\
\text { of life }\end{array}$ & $\begin{array}{l}\text { Participants, who were } \\
\text { persons living with } \\
\text { Alzheimer's disease whose } \\
\text { profiles differed notably } \\
\text { (age, cognitive and social } \\
\text { characteristics), found the } \\
\text { app beneficial. All of the } \\
\text { participants were able to } \\
\text { learn how to operate } \\
\text { AP@LZ's functions and to } \\
\text { use them in their activities } \\
\text { of daily living. }\end{array}$ \\
\hline
\end{tabular}




\begin{tabular}{|c|c|c|c|c|c|c|c|}
\hline$\#$ & $\begin{array}{l}\text { Authors } \\
\text { \& Year }\end{array}$ & Name of App & $\begin{array}{c}\text { Description of } \\
\text { App }\end{array}$ & $\begin{array}{c}\text { Targeted } \\
\text { Group }\end{array}$ & Design/Methods & $\begin{array}{l}\text { Domain } \\
\text { of App }\end{array}$ & Significant Findings \\
\hline 12 & $\begin{array}{l}\text { Kerkhof } \\
\text { et al., } \\
2019\end{array}$ & FindMyApps & $\begin{array}{l}\text { To help users find } \\
\text { apps for self- } \\
\text { management and } \\
\text { meaningful } \\
\text { activities that suit } \\
\text { individual needs, } \\
\text { wishes, and } \\
\text { abilities of people } \\
\text { living with } \\
\text { dementia. }\end{array}$ & $\begin{array}{l}\text { Persons } \\
\text { living with } \\
\text { mild } \\
\text { dementia }\end{array}$ & $\begin{array}{l}\text { The development was } \\
\text { inspired by user experience } \\
\text { design and Agile } \\
\text { methodology. } \\
\text { Developed using a "user- } \\
\text { participatory design" that } \\
\text { involved users (persons } \\
\text { living with dementia and } \\
\text { informal caregivers) and } \\
\text { experts (designers, } \\
\text { developers and researchers) } \\
\text { closely collaborating. }\end{array}$ & $\begin{array}{l}\text { Manage- } \\
\text { ment } \\
\text { Quality } \\
\text { of life }\end{array}$ & $\begin{array}{l}\text { The development team } \\
\text { gained an understanding of } \\
\text { issues regarding usefulness } \\
\text { and issues to increase user- } \\
\text { friendliness through the } \\
\text { user-participatory design. } \\
\text { The authors "succeeded in } \\
\text { making a workable tool } \\
\text { with an unambiguous } \\
\text { routing for finding apps, } \\
\text { which requires minimal } \\
\text { effort from the target } \\
\text { group to master" (p. 13). }\end{array}$ \\
\hline 13 & $\begin{array}{l}\text { Krauskopf } \\
\text { \& Golden, } \\
2016\end{array}$ & $\begin{array}{l}\text { Dementi- } \\
\text { Assist App }\end{array}$ & $\begin{array}{l}\text { To offer support } \\
\text { strategies for } \\
\text { caregivers to assist } \\
\text { them in dealing } \\
\text { with difficult } \\
\text { behaviours of } \\
\text { persons living with } \\
\text { dementia. } \\
\text { Contain the most } \\
\text { common } \\
\text { behavioural } \\
\text { expressions by the } \\
\text { persons living with } \\
\text { dementia, possible }\end{array}$ & $\begin{array}{l}\text { Family } \\
\text { caregivers }\end{array}$ & $\begin{array}{l}\text { Created by Baylor Scott \& } \\
\text { White Health, the largest } \\
\text { not-for-profit Texan } \\
\text { healthcare system. } \\
\text { Launched in March } 2015 . \\
\text { Incorporates the Positive } \\
\text { Physical Approach. }\end{array}$ & $\begin{array}{l}\text { Manage- } \\
\text { ment } \\
\text { Care } \\
\text { provision } \\
\text { Quality } \\
\text { of life }\end{array}$ & $\begin{array}{l}\text { Information provided is } \\
\text { practical. } \\
\text { Guides the caregiver to } \\
\text { manage difficult } \\
\text { behaviours of care } \\
\text { recipients with a positive } \\
\text { demeanour. } \\
\text { Described as user-friendly } \\
\text { and easy to navigate. }\end{array}$ \\
\hline
\end{tabular}




\begin{tabular}{|c|c|c|c|c|c|c|c|}
\hline$\#$ & $\begin{array}{l}\text { Authors } \\
\text { \& Year }\end{array}$ & Name of App & $\begin{array}{c}\text { Description of } \\
\text { App }\end{array}$ & $\begin{array}{c}\text { Targeted } \\
\text { Group }\end{array}$ & Design/Methods & $\begin{array}{c}\text { Domain } \\
\text { of App }\end{array}$ & Significant Findings \\
\hline & & & $\begin{array}{l}\text { causes, and } \\
\text { suggested actions } \\
\text { to take for the } \\
\text { behavioural } \\
\text { expression. }\end{array}$ & & & & \\
\hline 14 & $\begin{array}{l}\text { Meiland } \\
\text { et al., } \\
2012\end{array}$ & $\begin{array}{l}\text { COGKNO-W } \\
\text { Day } \\
\text { Navigator } \\
(\mathrm{CDN})\end{array}$ & $\begin{array}{l}\text { To support persons } \\
\text { living with mild } \\
\text { dementia in their } \\
\text { daily lives in terms } \\
\text { of memory, social } \\
\text { contacts, daily } \\
\text { activities, and } \\
\text { safety. }\end{array}$ & $\begin{array}{l}\text { Persons } \\
\text { living with } \\
\text { mild } \\
\text { dementia }\end{array}$ & $\begin{array}{l}\text { A user participatory method } \\
\text { was implemented, which } \\
\text { consisted of three one-year } \\
\text { cycles with field tests in } \\
\text { Amsterdam, Belfast, and } \\
\text { Lulea. } \\
\text { Persons living with } \\
\text { dementia and their } \\
\text { caregivers participated in } \\
\text { interviews, observations, } \\
\text { questionnaires, logging, and } \\
\text { diaries to collect data on } \\
\text { usability. }\end{array}$ & $\begin{array}{l}\text { Manage- } \\
\text { ment } \\
\text { Care } \\
\text { provision } \\
\text { Quality } \\
\text { of life }\end{array}$ & $\begin{array}{l}\text { Evaluation results showed } \\
\text { that participants found } \\
\text { CDN as user-friendly and } \\
\text { useful. }\end{array}$ \\
\hline 15 & $\begin{array}{l}\text { Moreira et } \\
\text { al., } 2013\end{array}$ & STAIz & $\begin{array}{l}\text { To provide } \\
\text { healthcare } \\
\text { providers with } \\
\text { relevant } \\
\text { information about } \\
\text { persons living with } \\
\text { dementia's } \\
\text { progress. }\end{array}$ & $\begin{array}{l}\text { Persons } \\
\text { living with } \\
\text { dementia } \\
\text { and their } \\
\text { caregivers }\end{array}$ & $\begin{array}{l}\text { Throughout the } \\
\text { development phase, } \\
\text { usability tests were } \\
\text { performed with caregivers } \\
\text { in order to evaluate the } \\
\text { app's usability. }\end{array}$ & $\begin{array}{l}\text { Manage- } \\
\text { ment } \\
\text { Care } \\
\text { provision }\end{array}$ & $\begin{array}{l}\text { Despite the app being in } \\
\text { the initial phases, the } \\
\text { results are promising in } \\
\text { that it can support the } \\
\text { diagnosis, tracking and } \\
\text { rehabilitation of persons } \\
\text { living with dementia as } \\
\text { well as provide }\end{array}$ \\
\hline
\end{tabular}




\begin{tabular}{|c|c|c|c|c|c|c|c|}
\hline$\#$ & $\begin{array}{l}\text { Authors } \\
\text { \& Year }\end{array}$ & Name of App & $\begin{array}{c}\text { Description of } \\
\text { App }\end{array}$ & $\begin{array}{l}\text { Targeted } \\
\text { Group }\end{array}$ & Design/Methods & $\begin{array}{l}\text { Domain } \\
\text { of App }\end{array}$ & Significant Findings \\
\hline & & & $\begin{array}{l}\text { To empower } \\
\text { persons living with } \\
\text { dementia with a } \\
\text { way to perform } \\
\text { cognitive } \\
\text { rehabilitation } \\
\text { exercises. }\end{array}$ & & & & $\begin{array}{l}\text { improvements in cognitive } \\
\text { rehabilitation. }\end{array}$ \\
\hline 16 & $\begin{array}{l}\text { Peisachov } \\
\text {-ich et al., } \\
2018\end{array}$ & SafeHome & $\begin{array}{l}\text { To train users to } \\
\text { identify common } \\
\text { safety hazards in } \\
\text { the home through } \\
\text { gaming. } \\
\text { The game presents } \\
\text { users with a } \\
\text { furnished digital } \\
\text { kitchen containing } \\
\text { potential hazards } \\
\text { and users must } \\
\text { identify hazards. } \\
\text { They are guided } \\
\text { through this game } \\
\text { with a mascot who } \\
\text { provides } \\
\text { encouragement and } \\
\text { explanations for } \\
\text { correct and }\end{array}$ & $\begin{array}{l}\text { Informal } \\
\text { caregivers }\end{array}$ & $\begin{array}{l}\text { Designed using best- } \\
\text { practice guidelines to ensure } \\
\text { the app content was of high } \\
\text { clinical quality. A content } \\
\text { expert, in collaboration with } \\
\text { a design team, informed the } \\
\text { process and the design } \\
\text { choices. The team drew on } \\
\text { gaming-design theory and } \\
\text { used interactive 3D game } \\
\text { technology. } \\
\text { An empirical usability } \\
\text { approach was employed. } \\
\text { Heuristic evaluation was } \\
\text { conducted by content } \\
\text { experts. Usability testing } \\
\text { with caregivers was } \\
\text { implemented where they } \\
\text { completed a questionnaire } \\
\text { and participated in focus }\end{array}$ & $\begin{array}{l}\text { Manage- } \\
\text { ment } \\
\text { Care } \\
\text { provision }\end{array}$ & $\begin{array}{l}\text { Participants' report of the } \\
\text { app was overwhelmingly } \\
\text { positive. } \\
\text { Majority }(71 \%) \text { of } \\
\text { responses indicated the } \\
\text { experience with the app as } \\
\text { "wonderful" and "almost } \\
\text { wonderful". } \\
\text { Majority ( } 71 \%) \text { of } \\
\text { responses indicated that } \\
\text { the end-user experience } \\
\text { was "satisfying" or "near } \\
\text { satisfying". }\end{array}$ \\
\hline
\end{tabular}




\begin{tabular}{|c|c|c|c|c|c|c|c|}
\hline$\#$ & $\begin{array}{l}\text { Authors } \\
\text { \& Year }\end{array}$ & Name of App & $\begin{array}{l}\text { Description of } \\
\text { App }\end{array}$ & $\begin{array}{l}\text { Targeted } \\
\text { Group }\end{array}$ & Design/Methods & $\begin{array}{l}\text { Domain } \\
\text { of App }\end{array}$ & Significant Findings \\
\hline & & & $\begin{array}{l}\text { incorrect } \\
\text { selections. }\end{array}$ & & $\begin{array}{l}\text { groups after using the app } \\
\text { for } 30 \text { minutes. }\end{array}$ & & \\
\hline 17 & $\begin{array}{l}\text { Pitts et al., } \\
2015\end{array}$ & $\begin{array}{l}\text { An adapted } \\
\text { version of the } \\
\text { Yammer app }\end{array}$ & $\begin{array}{l}\text { To support } \\
\text { reflective learning } \\
\text { to deliver more } \\
\text { person-centred } \\
\text { care. }\end{array}$ & $\begin{array}{l}\text { Formal } \\
\text { caregivers }\end{array}$ & $\begin{array}{l}\text { Design informed by an } \\
\text { existing model of computer- } \\
\text { support reflective learning } \\
\text { and instantiated with } \\
\text { findings from a pre-design } \\
\text { study in one residential } \\
\text { home. }\end{array}$ & $\begin{array}{l}\text { Manage- } \\
\text { ment } \\
\text { Care } \\
\text { provision } \\
\text { Quality } \\
\text { of life }\end{array}$ & $\begin{array}{l}\text { Use of the app both } \\
\text { motivated and increased } \\
\text { different styles of care note } \\
\text { recording. } \\
\text { Little reflective learning } \\
\text { was identified or reported. }\end{array}$ \\
\hline 18 & $\begin{array}{l}\text { Sasaki, } \\
2016\end{array}$ & $\begin{array}{l}\text { No name } \\
\text { mentioned: A } \\
\text { smart-phone } \\
\text { app provides } \\
\text { preventive } \\
\text { care for the } \\
\text { elderly with } \\
\text { dementia }\end{array}$ & $\begin{array}{l}\text { A mobile app in the } \\
\text { early stages of } \\
\text { development that } \\
\text { provides preventive } \\
\text { care and } \\
\text { information about } \\
\text { early signs of } \\
\text { dementia. }\end{array}$ & $\begin{array}{l}\text { General } \\
\text { Public }\end{array}$ & $\begin{array}{l}\text { Design informed by the } \\
\text { literature. }\end{array}$ & $\begin{array}{l}\text { Prevent- } \\
\text { ion }\end{array}$ & $\begin{array}{l}\text { The designed app is } \\
\text { expected to provide } \\
\text { preventive care for the } \\
\text { elderly. }\end{array}$ \\
\hline 19 & $\begin{array}{l}\text { Savita et } \\
\text { al., } 2018\end{array}$ & MyDem & $\begin{array}{l}\text { To help users in } \\
\text { recognizing } \\
\text { frequently } \\
\text { encountered } \\
\text { objects through } \\
\text { image processing } \\
\text { and to encourage } \\
\text { users in }\end{array}$ & $\begin{array}{l}\text { Persons } \\
\text { living with } \\
\text { dementia } \\
\text { and their } \\
\text { caregivers. }\end{array}$ & $\begin{array}{l}\text { Three major stages were } \\
\text { conducted: (1) data } \\
\text { collection where interviews } \\
\text { with persons living with } \\
\text { dementia and their } \\
\text { caregivers took place; (2) } \\
\text { prototype development; and } \\
\text { (3) validation. }\end{array}$ & $\begin{array}{l}\text { Manage- } \\
\text { ment }\end{array}$ & $\begin{array}{l}\text { All of the participants } \\
\text { agreed that they could } \\
\text { easily identify the intended } \\
\text { object with the help of } \\
\text { MyDem. }\end{array}$ \\
\hline
\end{tabular}




\begin{tabular}{|c|c|c|c|c|c|c|c|}
\hline \# & $\begin{array}{l}\text { Authors } \\
\text { \& Year }\end{array}$ & Name of App & $\begin{array}{c}\text { Description of } \\
\text { App }\end{array}$ & $\begin{array}{c}\text { Targeted } \\
\text { Group }\end{array}$ & Design/Methods & $\begin{array}{l}\text { Domain } \\
\text { of App }\end{array}$ & Significant Findings \\
\hline & & & $\begin{array}{l}\text { remembering their } \\
\text { past through past } \\
\text { memory narration } \\
\text { of old photos. }\end{array}$ & & $\begin{array}{l}\text { User testing was } \\
\text { implemented with four } \\
\text { persons living with } \\
\text { dementia in Alzheimer } \\
\text { Center Perak in Malaysia. }\end{array}$ & & $\begin{array}{l}\text { Being able to identify their } \\
\text { daily used items without } \\
\text { depending on their } \\
\text { caregiver made the } \\
\text { participants feel that the } \\
\text { app satisfied their needs. }\end{array}$ \\
\hline 20 & $\begin{array}{l}\text { Sindi et } \\
\text { al., } 2015\end{array}$ & $\begin{array}{l}\text { CAIDE } \\
\text { Dementia } \\
\text { Risk Score } \\
\text { App }\end{array}$ & $\begin{array}{l}\text { Based on the } \\
\text { CAIDE Dementia } \\
\text { Risk Score. } \\
\text { An evidence-based } \\
\text { tool that allows } \\
\text { users to detect their } \\
\text { individual risk, } \\
\text { provides guidance } \\
\text { for risk } \\
\text { modification, and } \\
\text { suggests consulting } \\
\text { a healthcare } \\
\text { practitioner if } \\
\text { required to discuss } \\
\text { appropriate } \\
\text { lifestyle } \\
\text { interventions to } \\
\text { reduce risk factors. }\end{array}$ & $\begin{array}{l}\text { General } \\
\text { public }\end{array}$ & $\begin{array}{l}\text { Authors collaborated with } \\
\text { Merz Pharmaceuticals } \\
\text { GmbH (Germany). }\end{array}$ & $\begin{array}{l}\text { Prevent- } \\
\text { ion } \\
\text { Diagnose }\end{array}$ & $\begin{array}{l}\text { First app of its kind to } \\
\text { predict the risk for } \\
\text { dementia through an } \\
\text { important evidence-based } \\
\text { tool. } \\
\text { Encourages users to } \\
\text { actively decrease their } \\
\text { modifiable risk factors and } \\
\text { postpone cognitive } \\
\text { impairment and dementia. }\end{array}$ \\
\hline 21 & $\begin{array}{l}\text { Solanas et } \\
\text { al., } 2013\end{array}$ & m-Carer & $\begin{array}{l}\text { Privately monitors } \\
\text { the location of } \\
\text { persons living with }\end{array}$ & $\begin{array}{l}\text { Persons } \\
\text { living with } \\
\text { dementia }\end{array}$ & Not mentioned. & $\begin{array}{l}\text { Manage- } \\
\text { ment }\end{array}$ & $\begin{array}{l}\text { A non-invasive tool aimed } \\
\text { at assisting caregivers to } \\
\text { supervise the regular }\end{array}$ \\
\hline
\end{tabular}




\begin{tabular}{|c|c|c|c|c|c|c|c|}
\hline$\#$ & $\begin{array}{l}\text { Authors } \\
\text { \& Year }\end{array}$ & Name of App & $\begin{array}{c}\text { Description of } \\
\text { App }\end{array}$ & $\begin{array}{c}\text { Targeted } \\
\text { Group }\end{array}$ & Design/Methods & $\begin{array}{c}\text { Domain } \\
\text { of App }\end{array}$ & Significant Findings \\
\hline & & & $\begin{array}{l}\text { dementia and } \\
\text { allows their safe } \\
\text { recovery if "they } \\
\text { get lost or } \\
\text { disoriented" (p. } \\
20 \text { ). }\end{array}$ & $\begin{array}{l}\text { and their } \\
\text { caregivers }\end{array}$ & & $\begin{array}{l}\text { Care } \\
\text { provision } \\
\text { Quality } \\
\text { of life }\end{array}$ & $\begin{array}{l}\text { activities of persons living } \\
\text { with dementia whilst } \\
\text { respecting their } \\
\text { fundamental right to } \\
\text { privacy. }\end{array}$ \\
\hline 22 & $\begin{array}{l}\text { Sposaro et } \\
\text { al., } 2010\end{array}$ & iWander & $\begin{array}{l}\text { Designed to } \\
\text { alleviate stress, } \\
\text { financial burden, } \\
\text { and offer easier } \\
\text { remote monitoring } \\
\text { for caregivers of } \\
\text { persons living with } \\
\text { dementia by } \\
\text { notifying the } \\
\text { caregiver of } \\
\text { wandering } \\
\text { behaviour. } \\
\text { Has Global } \\
\text { Positioning System } \\
\text { (GPS) and } \\
\text { communication } \\
\text { capabilities that } \\
\text { help navigate } \\
\text { persons living with } \\
\text { dementia to a safe } \\
\text { location, notify }\end{array}$ & $\begin{array}{l}\text { Persons } \\
\text { living with } \\
\text { dementia } \\
\text { and their } \\
\text { caregivers }\end{array}$ & $\begin{array}{l}\text { Designed by the authors } \\
\text { using the Bayesian network } \\
\text { techniques, which is a } \\
\text { model for determining the } \\
\text { probability that an event } \\
\text { occurs given other relevant } \\
\text { variables. }\end{array}$ & $\begin{array}{l}\text { Manage- } \\
\text { ment } \\
\text { Care } \\
\text { provision } \\
\text { Quality } \\
\text { of life }\end{array}$ & $\begin{array}{l}\text { More use of this app will } \\
\text { increase its accuracy. } \\
\text { Has a significant potential } \\
\text { for caregivers to cost- } \\
\text { effectively monitor } \\
\text { persons living with } \\
\text { dementia. }\end{array}$ \\
\hline
\end{tabular}




\begin{tabular}{|c|c|c|c|c|c|c|c|}
\hline$\#$ & $\begin{array}{l}\text { Authors } \\
\text { \& Year }\end{array}$ & Name of App & $\begin{array}{c}\text { Description of } \\
\text { App }\end{array}$ & $\begin{array}{c}\text { Targeted } \\
\text { Group }\end{array}$ & Design/Methods & $\begin{array}{l}\text { Domain } \\
\text { of App }\end{array}$ & Significant Findings \\
\hline & & & $\begin{array}{l}\text { caregivers, and } \\
\text { provide the current } \\
\text { location of persons } \\
\text { living with } \\
\text { dementia and call } \\
911 .\end{array}$ & & & & \\
\hline 23 & $\begin{array}{l}\text { Varshney } \\
\text { et al., } \\
2016\end{array}$ & AlzhaTV & $\begin{array}{l}\text { To allow family } \\
\text { members and } \\
\text { friends to stay } \\
\text { connected with } \\
\text { their loved ones } \\
\text { living with } \\
\text { dementia residing } \\
\text { at a nursing home } \\
\text { through making } \\
\text { and uploading } \\
\text { videos which are } \\
\text { instantly displayed } \\
\text { on the care } \\
\text { recipient's } \\
\text { television. }\end{array}$ & $\begin{array}{l}\text { Informal } \\
\text { caregivers }\end{array}$ & $\begin{array}{l}\text { Design informed by } \\
\text { literature. }\end{array}$ & $\begin{array}{l}\text { Manage- } \\
\text { ment } \\
\text { Care } \\
\text { provision } \\
\text { Quality } \\
\text { of life }\end{array}$ & $\begin{array}{l}\text { Preliminary responses of } \\
\text { care recipients have been } \\
\text { extremely positive and } \\
\text { family members are also } \\
\text { very satisfied. } \\
\text { Patients are showing some } \\
\text { improvements in their } \\
\text { symptoms as well as a } \\
\text { reduction of as-needed } \\
\text { medication use. }\end{array}$ \\
\hline 24 & $\begin{array}{l}\text { Zachos et } \\
\text { al., } 2013\end{array}$ & $\begin{array}{l}\text { The Carer } \\
\text { App }\end{array}$ & $\begin{array}{l}\text { To support } \\
\text { caregivers to } \\
\text { generate new ideas } \\
\text { to reduce } \\
\text { challenging } \\
\text { behaviours. }\end{array}$ & $\begin{array}{l}\text { Formal } \\
\text { caregivers }\end{array}$ & $\begin{array}{l}\text { Design informed by } \\
\text { observations of care work, } \\
\text { interviews with caregivers, } \\
\text { and pilot-testing in a } \\
\text { residential home in England. }\end{array}$ & $\begin{array}{l}\text { Manage- } \\
\text { ment } \\
\text { Care } \\
\text { provision }\end{array}$ & $\begin{array}{l}\text { Evaluation of the app in } \\
\text { the residential home } \\
\text { indicated that caregivers } \\
\text { were able to use the app to } \\
\text { deliver novel care as well } \\
\text { as generate, reflect on, and }\end{array}$ \\
\hline
\end{tabular}




\begin{tabular}{|c|c|c|c|c|c|c|c|}
\hline$\#$ & $\begin{array}{l}\text { Authors } \\
\text { \& Year }\end{array}$ & Name of App & $\begin{array}{c}\text { Description of } \\
\text { App }\end{array}$ & $\begin{array}{c}\text { Targeted } \\
\text { Group }\end{array}$ & Design/Methods & $\begin{array}{c}\text { Domain } \\
\text { of App }\end{array}$ & Significant Findings \\
\hline & & & & & & $\begin{array}{l}\text { Quality } \\
\text { of life }\end{array}$ & $\begin{array}{l}\text { improve ideas to reduce } \\
\text { challenging behaviours in } \\
\text { residents. }\end{array}$ \\
\hline 25 & $\begin{array}{l}\text { Zorluoglu } \\
\text { et al., } \\
2015\end{array}$ & $\begin{array}{l}\text { Mobile } \\
\text { Screening } \\
\text { Test }\end{array}$ & $\begin{array}{l}\text { A medium to } \\
\text { conduct neuro- } \\
\text { psychological tests } \\
\text { for early detection } \\
\text { of dementia. }\end{array}$ & $\begin{array}{l}\text { General } \\
\text { public }\end{array}$ & $\begin{array}{l}\text { Design informed by } 33 \\
\text { questions from } 14 \text { neuro- } \\
\text { psychological tests of } 8 \\
\text { different cognitive functions } \\
\text { (arithmetic, orientation, } \\
\text { abstraction, attention, } \\
\text { memory, language, visual, } \\
\text { and executive functions). }\end{array}$ & Diagnose & $\begin{array}{l}\text { The app could differentiate } \\
\text { people with healthy } \\
\text { cognitive abilities from } \\
\text { individuals who were } \\
\text { diagnosed with dementia } \\
\text { with statistical } \\
\text { significance. }\end{array}$ \\
\hline
\end{tabular}




\section{APPENDIX B}

\section{Literature Review Framework}

1. Provide a grounding question to guide the review.

2. Document the databases used.

3. Specify time limits applied to the search.

4. List the inclusion and exclusion criteria.

5. List the search terms used.

6. Document the search process.

7. Assess retrieved articles for relevance.

8. Provide a statement specifying the number of retrieved articles at the end of the search.

9. Conduct quality appraisal of retrieved literature.

10. Critically review the literature.

11. Check the reference list for accuracy.

Adapted from Kable, A. K., Pich, J., \& Maslin-Prothero, S. E. (2012). A structured approach to documenting a search strategy for publication: A 12 step guideline for authors. Nurse Education Today, 32(8), 878-886. 


\section{APPENDIX C}

Table of Inclusion and Exclusion Criteria Used for Literature Review Search

\begin{tabular}{|c|c|c|c|}
\hline & & Inclusion Criteria & Exclusion Criteria \\
\hline \multirow[t]{5}{*}{$\begin{array}{l}\text { Qualitative } \\
\text { Studies }\end{array}$} & $\begin{array}{l}\text { Population 1: } \\
\text { Family caregivers }\end{array}$ & $\begin{array}{l}\text { A relative/family } \\
\text { caregiver, such as spouse, } \\
\text { partner, sibling, son, } \\
\text { daughter, son-in-law, } \\
\text { daughter-in-law, cousin, } \\
\text { etc. }\end{array}$ & $\begin{array}{l}\text { Non-family informal } \\
\text { caregivers, such as } \\
\text { friends; Formal } \\
\text { caregivers, such as paid } \\
\text { caregivers, healthcare } \\
\text { professionals. }\end{array}$ \\
\hline & $\begin{array}{l}\text { Population 2: } \\
\text { People living with } \\
\text { dementia }\end{array}$ & $\begin{array}{l}\text { Any type of dementia that } \\
\text { is the primary and sole } \\
\text { issue under investigation. }\end{array}$ & $\begin{array}{l}\text { Dementia is a } \\
\text { secondary or joint issue } \\
\text { under investigation. }\end{array}$ \\
\hline & $\begin{array}{l}\text { Phenomena of } \\
\text { Interest: } \\
\text { Experience of caring } \\
\text { for a family member } \\
\text { living with dementia }\end{array}$ & $\begin{array}{l}\text { Studies that explore the } \\
\text { experience of caregiving } \\
\text { for a relative living with } \\
\text { dementia. }\end{array}$ & $\begin{array}{l}\text { Studies that do not } \\
\text { include the experience } \\
\text { of caregiving for a } \\
\text { relative living with } \\
\text { dementia. }\end{array}$ \\
\hline & $\begin{array}{l}\text { Phenomena of } \\
\text { Interest: } \\
\text { User experiences of } \\
\text { family caregivers of } \\
\text { Persons living with } \\
\text { dementia using } \\
\text { technology or } \\
\text { mobile apps in } \\
\text { caregiving activities }\end{array}$ & $\begin{array}{l}\text { Studies that explore the } \\
\text { user experiences of family } \\
\text { caregivers of persons } \\
\text { living with dementia } \\
\text { using technology or } \\
\text { mobile apps in caregiving } \\
\text { activities. }\end{array}$ & $\begin{array}{l}\text { Studies that do not } \\
\text { include the user } \\
\text { experiences of family } \\
\text { caregivers of persons } \\
\text { living with dementia } \\
\text { using technology or } \\
\text { mobile apps in } \\
\text { caregiving activities. }\end{array}$ \\
\hline & $\begin{array}{l}\text { Context: } \\
\text { Caring for a family } \\
\text { member living with } \\
\text { dementia who is } \\
\text { living in the } \\
\text { community }\end{array}$ & $\begin{array}{l}\text { Relative living with } \\
\text { dementia is residing in a } \\
\text { community setting/at } \\
\text { home. }\end{array}$ & $\begin{array}{l}\text { Relative living with } \\
\text { dementia is residing in } \\
\text { residential/nursing } \\
\text { home. } \\
\text { Relative living with } \\
\text { dementia is deceased. }\end{array}$ \\
\hline $\begin{array}{l}\text { Quantitative } \\
\text { Studies }\end{array}$ & $\begin{array}{l}\text { Population 1: } \\
\text { Family caregivers }\end{array}$ & $\begin{array}{l}\text { A relative/family } \\
\text { caregiver, such as spouse, } \\
\text { partner, sibling, son, } \\
\text { daughter, son-in-law, }\end{array}$ & $\begin{array}{l}\text { Non-family informal } \\
\text { caregivers, such as } \\
\text { friends; Formal } \\
\text { caregivers, such as paid }\end{array}$ \\
\hline
\end{tabular}




\begin{tabular}{|c|c|c|c|}
\hline & & $\begin{array}{l}\text { daughter-in-law, cousin, } \\
\text { etc. }\end{array}$ & $\begin{array}{l}\text { caregivers, healthcare } \\
\text { professionals. }\end{array}$ \\
\hline & $\begin{array}{l}\text { Population 2: } \\
\text { People living with } \\
\text { dementia }\end{array}$ & $\begin{array}{l}\text { Any type of dementia that } \\
\text { is the primary and sole } \\
\text { issue under investigation. }\end{array}$ & $\begin{array}{l}\text { Dementia is a } \\
\text { secondary or joint issue } \\
\text { under investigation. }\end{array}$ \\
\hline & $\begin{array}{l}\text { Intervention: } \\
\text { The use of a mobile } \\
\text { app }\end{array}$ & $\begin{array}{l}\text { Any mobile app designed } \\
\text { for dementia care, } \\
\text { particularly for family } \\
\text { caregivers of persons } \\
\text { living with dementia. }\end{array}$ & $\begin{array}{l}\text { Any mobile app not } \\
\text { designed for dementia } \\
\text { care. }\end{array}$ \\
\hline & Comparator: & & \\
\hline & $\begin{array}{l}\text { Non-use of a mobile } \\
\text { app or not applicable }\end{array}$ & & \\
\hline & $\begin{array}{l}\text { Outcome }(s) \text { of } \\
\text { Interest: } \\
\text { Improved caregiver } \\
\text { outcomes and/or } \\
\text { meeting the needs of } \\
\text { the caregiver }\end{array}$ & $\begin{array}{l}\text { Studies that evaluated the } \\
\text { impact of the designed } \\
\text { mobile app on improving } \\
\text { caregiver outcomes and/or } \\
\text { meeting their caregiving } \\
\text { needs. }\end{array}$ & $\begin{array}{l}\text { Studies that did not } \\
\text { evaluate the impact of } \\
\text { the designed mobile } \\
\text { app on improving } \\
\text { caregiver outcomes } \\
\text { and/or meeting their } \\
\text { caregiving needs. }\end{array}$ \\
\hline $\begin{array}{l}\text { Article } \\
\text { Characteristics }\end{array}$ & & $\begin{array}{l}\text { English language, full } \\
\text { text, studies published } \\
\text { from } 1997 \text { (inclusive) to } \\
\text { present, empirical studies } \\
\text { from any geographical } \\
\text { location, and peer- } \\
\text { reviewed. }\end{array}$ & $\begin{array}{l}\text { Non-English language, } \\
\text { no full text, studies } \\
\text { published before } 1997 .\end{array}$ \\
\hline $\begin{array}{l}\text { Grey } \\
\text { Literature } \\
\text { Characteristics }\end{array}$ & & $\begin{array}{l}\text { English language, full } \\
\text { text, literature published } \\
\text { from } 1997 \text { (inclusive) to } \\
\text { present, any geographical } \\
\text { location, and published by } \\
\text { reputable government, } \\
\text { healthcare, or non-profit } \\
\text { organizations. }\end{array}$ & $\begin{array}{l}\text { Non-English language, } \\
\text { no full text, studies } \\
\text { published before } 1997 .\end{array}$ \\
\hline
\end{tabular}




\section{APPENDIX D}

\section{PRISMA Flow Diagram}
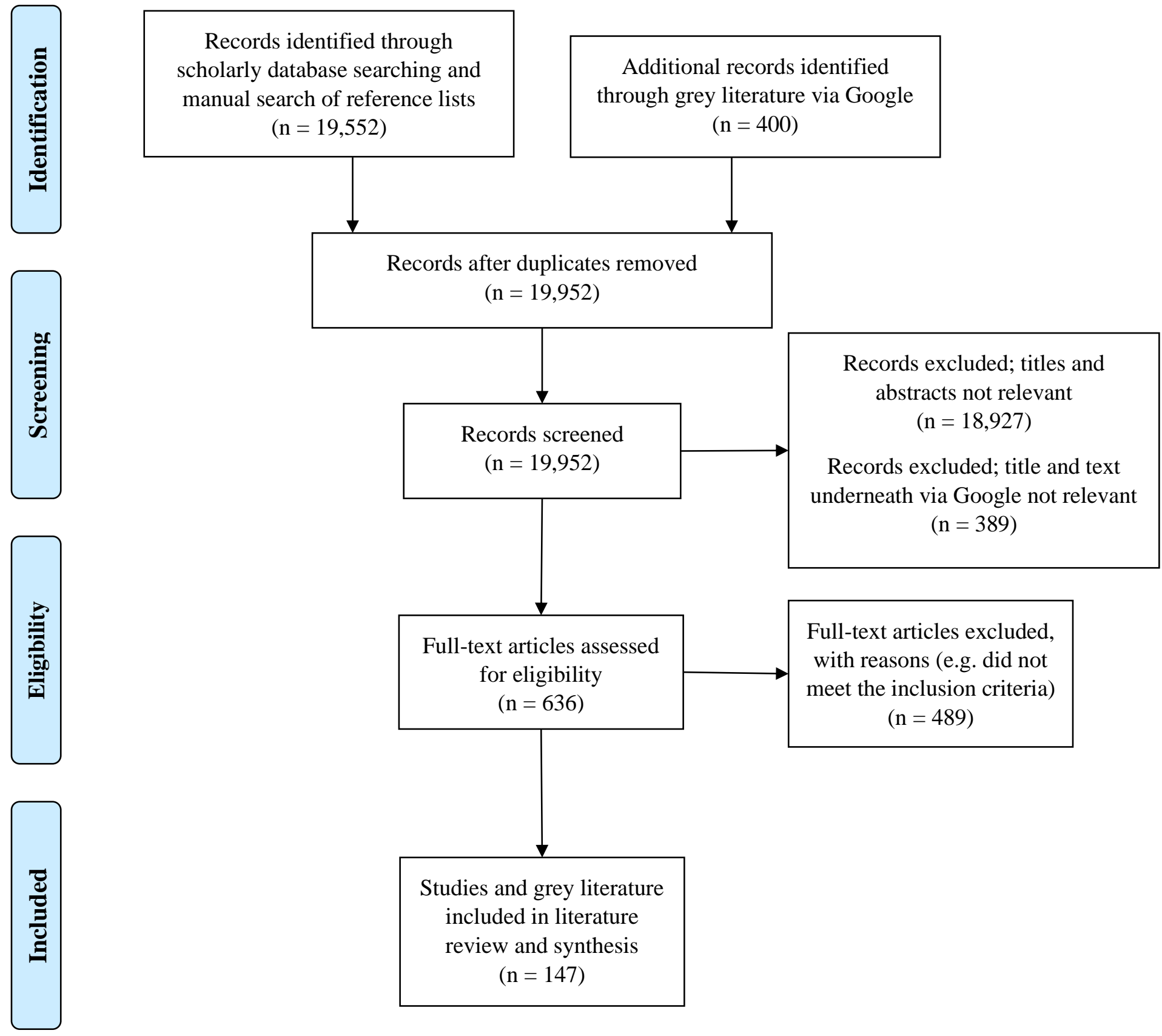

Adapted from: Moher, D., Liberati, A., Tetzlaff, J., Altman, D. G., \& The PRISMA Group. (2009). Preferred Reporting Items for Systematic Reviews and Meta-Analyses: The PRISMA Statement. PLOS Medicine, 6(6), e1000097. doi:10.1371/journal.pmed1000097 
APPENDIX E

Literature Review References

\begin{tabular}{cccc}
\hline $\begin{array}{c}\text { Article } \\
\text { Number }\end{array}$ & Author(s) & Year & Title \\
\hline
\end{tabular}

\section{Family Caregiving}

\begin{tabular}{|c|c|c|c|}
\hline 1. & Armstrong, P., \& Kits, O. & 2001 & One hundred years of caregiving. \\
\hline 2. & Bowers, B. J. & 1987 & $\begin{array}{l}\text { Intergenerational caregiving: Adult } \\
\text { caregivers and their aging parents. }\end{array}$ \\
\hline 3. & $\begin{array}{l}\text { British Columbia Law } \\
\text { Institute }\end{array}$ & n.d. & Chapter 2 - Family caregiving in BC. \\
\hline 4. & $\begin{array}{l}\text { Chappell, N. L., \& Funk, L. } \\
\text { M. }\end{array}$ & 2011 & Social support, caregiving, and aging. \\
\hline 5. & $\begin{array}{l}\text { Etters, L., Goodall, D., \& } \\
\text { Harrison, B. E. }\end{array}$ & 2008 & $\begin{array}{l}\text { Caregiver burden among dementia patient } \\
\text { caregivers: A review of the literature. }\end{array}$ \\
\hline 6. & Farran, C. J. & 2002 & $\begin{array}{l}\text { Family caregivers: A critical resource in } \\
\text { today's changing health-care climate. }\end{array}$ \\
\hline 7. & $\begin{array}{l}\text { Llanque, S., Savage, L., } \\
\text { Rosenburg, N., \& Caserta, } \\
\text { M. }\end{array}$ & 2016 & $\begin{array}{l}\text { Concept analysis: Alzheimer's caregiver } \\
\text { stress. }\end{array}$ \\
\hline 8. & $\begin{array}{l}\text { Schumacher, K., Beck, C. } \\
\text { A., \& Marren, J. M. }\end{array}$ & 2006 & $\begin{array}{l}\text { Family caregivers: Caring for older adults, } \\
\text { working with their families. }\end{array}$ \\
\hline 9. & Scott, L. D. & 2001 & $\begin{array}{l}\text { Technological caregiving: A qualitative } \\
\text { perspective. }\end{array}$ \\
\hline 10. & $\begin{array}{l}\text { Tam-Tham, H., Nettel- } \\
\text { Aguirre, A., Silvius, J., } \\
\text { Dalziel, W., Garcia, L., } \\
\text { Molnar, F., \& Drummond, } \\
\text { N. }\end{array}$ & 2016 & $\begin{array}{l}\text { Provision of dementia-related services in } \\
\text { Canada: A comparative study. }\end{array}$ \\
\hline 11. & $\begin{array}{l}\text { Yap, L. K. P., Seow, C. C. } \\
\text { D., Henderson, L. M., \& } \\
\text { Goh, Y. N. J. }\end{array}$ & 2005 & $\begin{array}{l}\text { Family caregivers and caregiving in } \\
\text { dementia. }\end{array}$ \\
\hline
\end{tabular}




\section{Experiences of Family Caregivers of Persons Living with Dementia}

$\begin{array}{lll}\text { 12. Alzheimer Society of } & 2010 & \text { Rising Tide: The impact of dementia on } \\ & \text { Canadian society. }\end{array}$

13. Andrén, S., \& Elmståhl, S. $2005 \quad$ Family caregivers’ subjective experiences of satisfaction in dementia care: Aspects of burden, subjective health and sense of coherence.

14. Ashwill, R., Mulhall, S.,

2015 Caregiving experience for people with Johnson, D. K., \& Galvin, J. E. Lewy Body Dementia: Spouse versus adult child.

15. Brodaty, H., \& Donkin, M. 2009 Family caregivers of people with dementia.

16. Brodaty, H., Thomson, C., 2005 Why caregivers of people with dementia Thompson, C., \& Fine, M. and memory loss don't use services.

17. Canadian Institute for

2018 Almost half of unpaid caregivers of seniors Health Information with dementia experience distress: New report paints national picture of dementia and its impact on health systems and Canadians.

18. Capistrant, B. D. 2016 Caregiving for older adults and the caregivers' health: An epidemiologic review.

19. Chappell, N. L., Dujela, C., 2014 \& Smith, A.

2013 Improving caregiving competence, stress

20. Chiu, M., Wesson, V., Sadavoy, J. coping, and mental well-being in informal dementia carers.

21. Chua, J., \& Pachana, N. A. 2016 Use of a psychoeducational skill training DVD program to reduce stress in Chinese Australian and Singaporean dementia caregivers: A pilot study.

22. Clyburn, L. D., Stones, M. $2000 \quad$ Predicting caregiver burden and depression J., Hadjistavropoulos, T., \& Tuokko, H.

23. Colantonio, A., Kositsky, A. 2001 J., Cohen, C., \& Vernich, L.

24. Conde-Sala, J. L., Garre- 2010a in Alzheimer's disease.

What support do caregivers of elderly want? Results from the Canadian Study of Health and Aging.

Olmo, J., Turró-Garriga, O.,

Differential features of burden between spouse and adult-child caregivers of 
Vilalta-Franch, J., \& LópezPousa, S.

25. Conde-Sala, J. L., GarreOlmo, J., Turró-Garriga, O., Vilalta-Franch, J., \& LópezPousa, S.

26. Connell, C. M., Janevic, M. R., \& Gallant, M. P.

27. Cooper, C., Katona, C., Orrell, M., \& Livingston, G.

28. Crespo, M., López, J., \& Zarit, S. H.

29. de Rotrou, J., Cantegreil, I., Faucounau, V., Wenisch, E., Chausson, C., Jegou, D., Grabar, S., \& Rigaud, A. S.

30. del-Pino-Casado, R., Cardosa, M. R., LópezMartínez, C., \& Orgeta, V.

31. Devor, M., \& Renvall, M. 2008

32. Di Mattei, V.E., Prunas, A., Novella, L., Marcone, A., Cappa, S. F., \& Sarno, L.

33. Eloniemi-Sulkava, U., Notkola, I. L., Hentinen, M., Kivela, S. L., Sivenius, J., \& Sulkava, R.

34. Forbes, D. A., Markle-Reid, 2008 M., Hawranik, P., Peacock, S., Kingston, D., Morgan, D., Henderson, S., Leipert, B., \& Jansen, S. L.

2001 patients with Alzheimer's disease: An exploratory comparative design.

2010b Quality of life of patients with Alzheimer's disease: Differential perceptions between spouse and adult child caregivers.

The costs of caring: Impact of dementia on family caregivers.

2008 Coping strategies, anxiety and depression in caregivers of people with Alzheimer's disease.

2005 Depression and anxiety in primary caregivers: A comparative study of caregivers of demented and nondemented older persons.

2011 Do patients diagnosed with Alzheimer's disease benefit from a psycho-educational programme for family caregivers? A randomised controlled study.

2019 The association between subjective caregiver burden and depressive symptoms in carers of older relatives: A systematic review and meta-analysis.

An educational intervention to support caregivers of elders with dementia.

2008 The burden of distress in caregivers of elderly demented patients and its relationship with coping strategies.

2001 Effects of supporting community-living demented patients and their caregivers: A randomized trial.

Availability and acceptability of Canadian home and community-based services: Perspectives of family caregivers of persons with dementia. 
35. Gallagher, D., Mhaolain, A. 2011 N., Crosby, L., Ryan, D., Lacey, L., Coen, R. F., Walsh, C., Coakley, D., Walsh, J. B., Cunningham, C., \& Lawlor, B. A.

36. Gottlieb, B. H., \& Wolfe, J. 2002

37. Hebert, R., Dubois, M. F., Wolfson, C., Chambers, L., \& Cohen, $\mathrm{C}$.

38. Hong, G. R. S., \& Kim, H. 2008

39. Hsu, C. C., Wang, Y. M., Huang, C. R., Sun, F. J., Lin, J. P., Yip, P. K., \& Liu, S. I.

40. Hunt, C. K.

41. Karg, N., Graessel, E., Randzio, O., \& Pendergrass, A.

42. Kim, H., Chang, M., Rose, 2012 K., \& Kim, S.

43. Kneebone, I. I., \& Martin, 2003 P. R.

44. Lai, D. W. L., \& Surood, S. 2008

45. Lee, Y., \& Smith, L.

46. Lewis, M. L., Hobday, J. V., 2010 \& Hepburn, K. W.
Self-efficacy for managing dementia may protect against burden and depression in Alzheimer's caregivers.

Coping with family caregiving to persons with dementia: A critical review.

Factors associated with long-term institutionalization of older people with dementia: Data from the Canadian Study of Health and Aging.

Family caregiver burden by relationship to care recipient with dementia in Korea.

2017 Sustained benefit of a psycho-educational training program for dementia caregivers in Taiwan.

Concepts in caregiver research.

Dementia as a predictor of care-related quality of life in informal caregivers: A cross-sectional study to investigate differences in health-related outcomes between dementia and non-dementia caregivers.

Predictors of caregiver burden in caregivers of individuals with dementia.

Coping and caregivers of people with dementia.

Service barriers of Chinese family caregivers in Canada.

Qualitative research on Korean American dementia caregivers' perception of caregiving: Heterogeneity between spouse caregivers and child caregivers.

Internet-based program for dementia caregivers. 
47. Lilly, M. B., Robinson, C. A., Holtzman, S., \& Bottorff, J.

48. Lloyd, J., Patterson, T., \& Muers, J.

49. Lwi, S. J., Ford, B. Q., Casey, J. J., Miller, B. L., \& Levenson, R. W.

50. Madaleno, T. R., Moriguti, J. C., Ferriolli, E., De Carlo, M. M. R., \& Lima, N. K.

51. Mavounza, C., Ouellet, M. 2018 C., \& Hudon, C.

52. Milne, A., Guess, R., \& Russ, A.

53. Morgan, D. G., Semchuk, K. M., Stewart, N. J., \& D'arcy, C.

54. Novais, T., Dauphinot, V., Krolak-Salmon, P., \& Mouchoux, C.

55. Ory, M., Hoffman, P., Yee, J., Tennstedt, S., \& Schulz, $\mathrm{R}$.

56. Ott, C. H., Sanders, S., \& Kelber, S. T.
2012 Can we move beyond burden and burnout to support the health and wellness of family caregivers to persons with dementia? Evidence from British Columbia, Canada.

2016 The positive aspects of caregiving in dementia: A critical review of the qualitative literature.

2017 Poor caregiver mental health predicts mortality of patients with neurodegenerative disease.

2019 Mood, lifestyle and cardiovascular risk factors among older caregivers of patients with Alzheimer's disease dementia: A case-control study.

Caregivers' emotional distress due to neuropsychiatric symptoms of persons with amnestic mild cognitive impairment or Alzheimer's disease.

2014 Pyscho-educational support for relatives of people with a recent diagnosis of mild to moderate dementia: An evaluation of a 'Course for Carers'.

2002 Rural families caring for a relative with dementia: Barriers to use of formal services.

2017 How to explore the needs of informal caregivers of individuals with cognitive impairment in Alzheimer's disease or related diseases? A systematic review of quantitative and qualitative studies.

1999 Prevalence and impact of caregiving: A detailed comparison between dementia and nondementia caregivers.

Grief and personal growth experience of spouses and adult-child caregivers of individuals with Alzheimer's disease and related dementias. 
57. Papastavrou, E.,

Kalokerinou, A.,

Papacostas, S. S., Tsangari, H., \& Sourtzi, P.

58. Peacock, S., Forbes, D., Markle-Reid, M., Hawranik, P., Morgan, D., Jansen, L., Leipert, B. D., \& Henderson, S. R.

59. Pinquart, M., \& Sörensen, S.

60. Quinn, C., Nelis, S. M., Martyr, A., Morris, R. G., Victor, C., \& Clare, L.

61. Reed, C., Belger, M., Dell'Agnello, G., Wimo, A., Argimon, J. M., Bruno, G., Dodel, R., Haro, J. M., Jones, R. W. \& Vellas, B.

62. Rinaldi, P., Spazzafumo, L. Mastriforti, R., Mattioli, P., Marvardi, M., Polidori, M. C., Abate G, Bartorelli, L., Bonaiuto, S., Capurso, A., Cucinotta, D., Gallucci, M., Giordano, M., Martorelli, M., Masaraki, G., Nieddu, A., Pettenati, C., Putzu, P., Tammaro, V.A,, Tomassini, P.F., Vergani, C., Senin, U., $\&$ Capurso, A.

63. Schoenmakers, B., Buntinx, F., \& Delepeleire, J.

64. Shim, B., Barroso, J., \& Davis, L. L.
2007 Caring for a relative with dementia: Family caregiver burden.

2010 The positive aspects of the caregiving journey with dementia: Using a strengthsbased perspective to reveal opportunities.

2011 Spouses, adult children, and children-inlaw as caregivers of older adults: A metaanalytic comparison.

2019 Caregiver influences on 'living well' for people with dementia: Findings from the IDEAL study.

2014 Caregiver burden in Alzheimer's disease: differential associations in adult-child and spousal caregivers in the GERAS observational study.

2005 Predictors of high level of burden and distress in caregivers of demented patients: Results of an Italian multicenter study.

2010

Factors determining the impact of caregiving on caregivers of elderly patients with dementia. A systematic literature review.

2012 A comparative qualitative analysis of stories of spousal caregivers of people with dementia: Negative, ambivalent, and positive experiences. 
65. Springate, B. A., \&

Tremont, G.

66. Stall, N. M., Kim, S. J., Hardacre, K. A., Shah, P. S., Straus, S. E., Bronskill, S. E., Lix, L. M., Bell, C. M., \& Rochon, P. A.

67. Stewart, M., Barnfather, A., 2006

Neufeld, A., Warren, S., Letourneau, N., \& Liu, L.

68. Sun, F.

69. Tremont, G., Davis, J. D., Bishop, D. S., \& Fortinsky, R. H.

70. Vandepitte, S., Putman, K., Van Den Noortgate, N., Verhaeghe, S., Mormont, E., Van Wilder, L., De Smedt., D., \& Annemans, L.

71. Van Mierlo, L. D., Meiland, 2011 F. J. M., Van der Roest, H. G., \& Droes, R. M.

72. Ward-Griffin, C., Hall, J., DeForge, R., St-Amant, O., McWilliam, C., Oudshoorn, A., Forbes, D., \& Klosek, M.

73. Yu, S. F. D., Cheng, S. T., 2018 \& Wang, J.
2012 Dimensions of caregiver burden in dementia: Impact of demographic, mood, and care recipient variables.

2019 Association of informal caregiver distress with health outcomes of communitydwelling dementia care recipients: A systematic review.

Accessible support for family caregivers of seniors with chronic conditions: From isolation to inclusion.

2014 Caregiving stress and coping: A thematic analysis of Chinese family caregivers of persons with dementia.

2008 Telephone-delivered psychosocial intervention reduces burden in dementia caregivers.

2018 Factors associated with the caregivers' desire to institutionalize persons with dementia: A cross-sectional study.

Personalised caregiver support: Effectiveness of psychosocial interventions in subgroups of caregivers of people with dementia.

2012 Dementia home care resources: How are we managing?

Unravelling positive aspects of caregiving in dementia: An integrative review of research literature.

\section{Technology and Caregiving}

74. Benefield, L. E., \& Beck, C. $2007 \quad \begin{aligned} & \text { Reducing the distance in distance- } \\ & \text { caregiving by technology innovation. }\end{aligned}$

75. Bier, N., Paquette, G., \& 2018 Smartphone for smart living: Using new Macoir, J. 
76. Boots, L. M., de Vugt, M. 2014 E., van Knippenberg, R. J., Kempen, G. I. J. M., \& Verhey, F. R. J.

77. Buettner, L. L., Yu, F., \& 2010 Burgener, S. C.

78. Godwin, K. M., Mills, W. L., Anderson, J. A., \& Kunik, M. E.

79. Hopwood, J., Walker, N., McDonagh, L., Rait, G., Walters, K., Iliffe, S., Ross, J., \& Davies, N.

80. Imbeault, H., Langlois, F., Bocti, C., Gagnon, L., \& Bier, N.

81. Kerkhof, Y. J. F., Graff, M. 2016 J. L., Bergsma, A., de Vocht, H. H. M., \& Droes, R.

82. Klimova, B., Valis, M., \& Kuca, K.

83. Kong, A. P.

2015

84. Lapid, M. I., Atherton, P. J., 2015 Clark, M. M., Kung, S., Sloan, J. A., \& Rummans, T. A.

85. Lee, E. technologies to cope with everyday limitations in semantic dementia.

A systematic review of Internet-based supportive interventions for caregivers of patients with dementia.

Evidence supporting technology-based interventions for people with early-stage Alzheimer's Disease.

2013 Technology-driven interventions for caregivers of persons with dementia: A systematic review.

2018 Internet-based interventions aimed at supporting family caregivers of people with dementia: Systematic review.

2018 Can people with Alzheimer's disease improve their day-to-day functioning with a tablet computer?

Better self-management and meaningful activities thanks to tablets? Development of a person-centered program to support people with mild dementia and their carers through use of hand-held touch screen devices.

2018 Exploring assistive technology as a potential beneficial intervention tool for people with Alzheimer's disease-a systematic review.

Conducting cognitive exercises for early dementia with the use of apps on iPads.

Cancer caregiver: Perceived benefits of technology.

2015 Do technology-based support groups reduce care burden among dementia caregivers? A review. 
86. Lee, J. A., Nguyen, H.,

Park, J., Tran, L., Nguyen,

T., \& Huynh, Y.

87. Lorenz, K., Freddolino, P. P., Comas-Herrera, A., Knapp, M., \& Damant, J.

88. Mao, H. F., Chang, L. H., Yao, G., Chen, W. Y., \& Huang, W. N. W.

89. Maresova, P., Tomsone, S., Lameski, P., Madureira, J., Mendes, A., Zdravevski, E., Chorbev, I., Trajkovik, V., Ellen, M., \& Rodil, K.

90. Martin, F., Turner, A., Wallace, L. M., \& Bradbury, N.

91. Nygard, L.

92. Nygard, L., \& Starkhammar, S.

93. Peterson, K., Hahn, H., Lee, A. J., Madison, C. A., \& Atri, A.

94. Powell, J., Chiu, T., \& Eysenbach, G.

95. Shreve, J., Baier, R., Epstein-Lubow, G., \& Gardner, R. L.

96. Silva, B. M., Rodrigues, J. J., de la Torre Díez, I., López-Coronado, M., \& Saleem, K.
2017 Usages of computers and smartphones to develop dementia care education program for Asian American family caregivers.

2019 Technology-based tools and services for people with dementia and carers: Mapping technology onto the dementia care pathway.

2015 Indicators of perceived useful dementia care assistive technology: Caregivers' perspectives.

2018 Technological solutions of older people with Alzheimer's disease: Review.

2013 Conceptualisation of self-management intervention for people with early stage dementia.

2008 The meaning of everyday technology as experienced by people with dementia who live alone.

2007 The use of everyday technology by people with dementia living alone: Mapping out the difficulties.

2016 In the information age, do dementia caregivers get the information they need? Semi-structured interviews to determine informal caregivers' education needs, barriers, and preferences.

2008 A systematic review of networked technologies supporting carers of people with dementia.

2016 Dementia caregivers' technology preferences: Design insights from qualitative interviews.

2015 Mobile-health: A review of current state in 2015. 
97. Smith, S. K., \& Mountain, G. A.

98. Topo, P.

99. Torp, S., Hanson, E., Hauge, S., Ulstein, I., \& Magnusson, L.

100. Tyack, C., \& Camic, P. M.

101. Washington, K. T., Meadows, S. E., Elliot, S. G., \& Koopman, R. J.

102. Zhao, Y., Feng, H., Hu, M., Hu, H., Li, H., Ning, H., Chen, H., Liao, L., \& Peng, L.
2012 New forms of information and communication technology (ICT) and the potential to facilitate social and leisure activity for people living with dementia.

2009 Technology studies to meet the needs of people with dementia and their caregivers.

2008 A pilot study how information and communication technology may contribute to health promotion among elderly spousal carers in Norway.

2017 Touchscreen interventions and the wellbeing of people with dementia and caregivers: A systematic review.

2011 Information needs of informal caregivers of older adults with chronic health conditions.

2019 Web-based interventions to improve mental health in home caregivers of people with dementia: Meta-analysis.

\section{Mobile Apps}

103. Abu-Dalbouh, H., Al- $2015 \quad$ A mobile reminder system for elderly and

Habeeb, A., Al-Kholifi, A., Alzheimer's patients.

Al-Motiry, I., \& Al-

Buhairy, $\mathrm{M}$.

104. Acharya, M. H., Gokani, T. 2017 Android application for dementia patient. B., Chauhan, K. N., \& Pandya, B. P.

105. Bhattacharyya, S., Benbow, 2017 S. M., \& Collins, E.

106. Brown, E. L., Ruggiano, N., 2016 Roberts, L., Hristidis, V., Whiteman, K. L., Castro, J., \& Page, T. F.

107. Burns, K., Jayasinha, R., \& Brodaty, H.
2017

Evaluation of an electronic App developed to assist clinicians in the management of behavioral and psychological symptoms of dementia (BPSD). 
108. Cook, B., \& Twidle, P.

109. Davis, B., Nies, M., Shehab, 2014 M., \& Shenk, D.

110. Docking, R. E., Lane, M., \& 2017 Schofield, P. A.

111. Filoteo, J. V., Cox, E. M., 2018 Split, M., Gross, M., Culjat, M., \& Keene, D.

112. Halbach, T., Solheim, I., Ytrehus, S., \& Schulz, T.

113. Imbeault, H., Gagnon, L., Pigot, H., Giroux, S., Marcotte, N., CribierDelande, P., Duval, J., Bocti, C., Lacombe, G., Fulop, T., \& Bier, N.

114. Kerkhof, Y., Pelgrum-

Keurhorst, M.,

Mangiaracina, F., Bergsma, A., Vrauwdeunt, G., Graff, M., \& Dröes, R. M.

115. Krauskopf, P. B., \& Golden, 2016 A.

116. Meiland, F. J., Bouman, A. I., Sävenstedt, S., Bentvelzen, S., Davies, R. J., Mulvenna, M. D., Nugent, C. D., Moelaert, F., Hettinga, M. E., Bengtsson, J. E., \& Dröes, R. M.

117. Moreira, H., Oliveira, R., \& 2013 Flores, N.
2016 Increasing awareness of Alzheimer's Disease through a mobile game.

Developing a pilot e-mobile app for dementia caregiver support: Lessons learned.

Usability testing of the iPhone app to improve pain assessment for older adults with cognitive impairment (prehospital setting): A qualitative study.

Evaluation of ReminX as a behavioral intervention for mild to moderate dementia.

2018 A mobile application for supporting dementia relatives: A case study.

2018 Impact of AP@LZ in the daily life of three persons with Alzheimer's disease: Long-term use and further exploration of its effectiveness.

2019 User-participatory development of FindMyApps; A tool to help people with mild dementia find supportive apps for self-management and meaningful activities.

AIDSInfo HIV/AIDS Guidelines App and DementiAssist App.

2012 Usability of a new electronic assistive device for community-dwelling persons with mild dementia.

STAlz: Remotely supporting the diagnosis, tracking and rehabilitation of patients with Alzheimer's. 
118. Peisachovich, E., Jokel, R., Appel, L., Da Silva, C., Sinclair, D., \& Soroor, W.

119. Pitts, K., Pudney, K., Zachos, K., Maiden, N., Krogstie, B., Jones, S., Rose, M., MacManus, J., \& Turner, I.

120. Sasaki, H.

121. Savita, K. S., Marrima, J. J. D. A., Muniandy, M., Abidin, A. I. Z., \& Taib, S.

M.

122. Sindi, S., Calov, E., Fokkens, J., Ngandu, T., Soininen, H., Tuomilehto, J., \& Kivipelto, M.

123. Solanas, A., Martinez-

Balleste, A., Perez-

Martinez, P. A., de la Pena, A. F., \& Ramos, J.

124. Sposaro, F., Danielson, J., \& Tyson, $\mathrm{G}$.

125. Varshney, S., Varshney, J., Varshney, U., \& Varshney, $\mathrm{S}$.

126. Zachos, K., Maiden, N., Pitts, K., Jones, S., Turner, I., Rose, M., Pudney, K., \& MacManus, J.

127. Zorluoglu, G., Kamasak, M. 2015

E., Tavacioglu, L., \&

Ozanar, P. O.

2018

2016
2018 SafeHome app: Design and usability for a digital application to promote physical safety to persons living with dementia.

2015 Using mobile devices and apps to support reflective learning about older people with dementia.

2016 A smartphone app provides preventive care for the elderly with dementia.

Help me! MyDem application for early stage dementia patients.

2015 The CAIDE Dementia Risk Score App: The development of an evidence-based mobile application to predict the risk of dementia.

2013 m-Carer: Privacy-aware monitoring for people with mild cognitive impairment and dementia.

iWander: An Android application for dementia patients.

AlzhaTV: A smart phone app for managing depression, anxiety, and agitation in nursing home dementia patients.

2013 A software app to support creativity in dementia care.

A mobile application for cognitive screening of dementia.

\section{User Experience}

128. Anderson, K., Burford, O., $2016 \quad \begin{aligned} & \text { Mobile health apps to facilitate self-care: A } \\ & \text { qualitative study of user experiences. }\end{aligned}$
\& Emmerton, L.


129. Bateman, D. R., Srinivas, B., Emmett, T. W., Schleyer, T. K., Holden, R. J., Hendrie, H. C., \& Callahan, C. M.

130. Colantonio, Cohen, \& Pon

131. Czarnuch, S., \& Mihailidis, A.

132. Davis, S. W., \& OakleyGirvan, I.

133. Forlizzi, J., \& Battarbee, K.

134. Gibson, G., Dickinson, C., Brittain, K., \& Robinson, L.

135. Holthe, T., Jentoft, R., Arntzen, C., \& Thorsen, K.

136. International Organization for Standardization

137. Khan, K.

138. Laidlaw, R., Dixon, D., Morse, T., Beattie, T. K., Kumwenda, S., \& Mpemberera, G.

139. Law, E. L. C., Roto, V., Hassenzahl, M., Vermeeren, A. P., \& Kort, J.
2017 Categorizing health outcomes and efficacy of mHealth apps for persons with cognitive impairment: A systematic review.

2001 Assessing support needs of caregivers of persons with dementia: Who wants what?

2011 The design of intelligent in-home assistive technologies: Assessing the needs of older adults with dementia and their caregivers.

2017 Achieving value in mobile health applications for cancer survivors.

2004 Understanding experience in interactive systems.

2015 The everyday use of assistive technology by people with dementia and their family carers: A qualitative study.

2018 Benefits and burdens: Family caregivers' experiences of assistive technology (AT) in everyday life with persons with youngonset dementia (YOD).

2010

ISO 9241-210:2010. Ergonomics of human-system interaction - Part 210: Human-centred design for interactive systems.

2012 User experience in mobile phones by using semantic differential methodology.

2017 Using participatory methods to design an mHealth intervention for a low income country, a case study in Chikwawa, Malawi.

2009 Understanding, scoping and defining user experience: A survey approach. 
140. Liu, L., Miguel Cruz, A., Rios Rincon, A., Buttar, V., Ranson, Q., \& Goertzen, D.

141. Mahoney, D. F.

2010

142. Park, J., Han, S. H., Kim, H. 2013 K., Oh, S., \& Moon, H.

143. Rathnayake, S., Moyle, W., 2018 Jones, C., \& Calleja, P.

144. Rialle, V., Ollivet, C., Guigui, C., \& Hervé, C.

145. Rosenberg, L., Kottorp, A., \& Nygård, L.

146. Ruggiano, N., Brown, E. L., 2019 Shaw, S., Geldmacher, D., Clarke, P., Hristidis, V., \& Bertram, J.

147. Tamim, S. R., \& Grant, M. 2016 M.
2015 What factors determine therapists' acceptance of new technologies for rehabilitation-a study using the Unified Theory of Acceptance and Use of Technology (UTAUT).

An evidence-based adoption of technology model for remote monitoring of elders' daily activities.

Modeling user experience: A case study on a mobile device.

mHealth applications as an educational and supportive resource for family carers of people with dementia: An integrative review.

2008 What do family caregivers of Alzheimer's disease patients desire in smart home technologies?

2012 Readiness for technology use with people with dementia: The perspectives of significant others.

The potential of information technology to navigate caregiving systems: Perspectives from dementia caregivers.

Exploring how health professionals create eHealth and mHealth education interventions. 


\section{APPENDIX F: RECRUITMENT DOCUMENTS}

\section{Recruitment Email}

Dear [Insert name/organization],

My name is Angel Wang and I am a Master of Nursing student at Ryerson University in the thesis stream. As part of the program requirements, I am conducting a research study about family caregivers' experiences of using mobile applications (apps on their cell phones) in their caregiving role of a relative living with dementia.

Would you consider assisting me to recruit participants for this study? Note: the study has received approval from the Research Ethics Board at Ryerson University and is being conducted under the supervision of Dr. Kristine Newman and Dr. Lori Schindel Martin.

The study will involve interviewing five to ten family caregivers who care for a relative living with dementia and use mobile apps in caregiving activities. My review of the literature showed that mobile technologies, particularly mobile apps, are a cost-effective and accessible method to assist family caregivers and improve their well-being. The findings of this study will offer an understanding of family caregivers' experiences of using mobile apps to support caregiving for a loved one living with dementia. This in turn can inform future mobile app developments to address caregiver needs of family caregivers and promote more effective caregiving and improved quality of life for care recipients and their family caregivers.

Attached in this email are the email message and recruitment poster that you can forward to members of your organization or who you think would be interested in participating. Should you encounter any inquiries about the study from interested participants, please direct these individuals to contact me directly via email at he.wang@ ryerson.ca. Feel free to contact me if you have any further questions about the study.

Thank you very in much in advance for your time and assistance. It is much appreciated.

Kind regards, Angel Wang, RN, BScN, MN Student

Daphne Cockwell School of Nursing, Ryerson University

Principal Investigator 


\section{Email Recruitment Message}

You're invited to participate in a research study!

My name is Angel Wang and I am a Master of Nursing student at Ryerson University in the thesis stream. I am writing to let you know about the opportunity to participate in my thesis study, titled: "Using mobile apps in caregiving: The user experiences of family caregivers of a relative living with dementia".

The purpose of this study is to explore the experiences of family caregivers of a relative living with dementia who uses mobile applications (apps) in their caregiving role. This in turn can inform future mobile app developments to address caregiver needs of family caregivers and promote more effective caregiving and improved quality of life for care recipients and their family caregivers.

Participation is voluntary and is open to anyone who meets the following criteria:

- 18 to 55 years-old;

- Able to understand and provide consent in English;

- Self-identifies as an adult child family caregiver (i.e. child or grandchild) of a relative living with dementia;

- Own a mobile device (e.g. smartphone, tablet); and

- Have prior experience using mobile apps to assist in caregiving for a relative living with dementia within the last 12 months (e.g. medication reminders, "brain-training" games, calendars, social network platform).

\section{WHAT WILL YOU BE ASKED TO DO}

1. Complete a short demographic form.

2. Participate in two in-person interviews at a private location of your choosing.

3. Take pictures with your mobile device.

\section{THANK YOU}

To thank you for your participation in the study, you will be offered a $\$ 20$ gift card.

If you would like more information or are interested in participating, please contact me at he.wang@ ryerson.ca and I will be happy to answer any questions you have about the study.

This study has been approved by the Ryerson University Research Ethics Board.

Sincerely,

Angel Wang, RN, BScN, MN Student

Ryerson University

Principal Investigator 


\title{
PARTICIPANTS NEEDED FOR CAREGIVING RESEARCH!
}

\section{Do you use mobile apps to assist with caregiving for your parent(s) or grandparent(s) living with dementia?}

If so, you may be eligible to take part in this study, which will explore the user experiences of family caregivers of a relative

living with dementia who use mobile apps* in caregiving.

*Mobile apps such as medication reminders, appointment

trackers/calendars, "brain-training" games, and social media platforms.

If you volunteer, your participation will include:

(1) Two interviews either in person or through the phone

(2) Take pictures with your mobile device

\section{Participants will receive a $\$ 10$ gift card after each interview in appreciation of their time.}

To participate in the study or for more information, please contact:

\author{
Principal Investigator \\ Angel Wang, RN, MN Student \\ Ryerson University \\ Email: he.wang@ryerson.ca
}




\section{Recruitment Posts on Social Media}

\section{Tweet on Twitter}

Character limit: 280; character count: 280

Hi [Insert name/organization], my name is Angel Wang \& I'm a graduate student at Ryerson University recruiting participants for my research about mobile apps \& \#dementia. Would you share/re-tweet the study's poster to your members or whoever you think would be interested? Thank you in advance!

\section{Facebook message}

Hi [Insert name/organization],

My name is Angel Wang and I am a Master of Nursing student at Ryerson University in the thesis stream. As part of the program requirements, I am conducting a research study about family caregivers' experiences of using mobile applications (apps on their cell phones) in their caregiving role of a relative living with dementia.

Would you consider assisting me to recruit participants for this study? Note: the study has received approval from the Research Ethics Board at Ryerson University and is being conducted under the supervision of Dr. Kristine Newman and Dr. Lori Schindel Martin.

The study will involve interviewing five to ten family caregivers who care for a relative living with dementia and use mobile apps in caregiving activities. My review of the literature showed that mobile technologies, particularly mobile apps, are a cost-effective and accessible method to assist family caregivers and improve their well-being. The findings of this study will offer an understanding of family caregivers' experiences of using mobile apps to support caregiving for a loved one living with dementia. This in turn can inform future mobile app developments to address caregiver needs of family caregivers and promote more effective caregiving and improved quality of life for care recipients and their family caregivers.

Attached in this message is the recruitment poster that you can post on your Facebook page/group to share to members of your organization. Should you encounter any inquiries about the study from interested participants, please direct these individuals to contact me directly via email at he.wang@ ryerson.ca. Feel free to contact me if you have any further questions about the study.

Thank you very much in advance for your time and assistance. It is much appreciated.

Kind regards, Angel Wang, RN, MN Student

Ryerson University

Principal Investigator 


\section{Facebook posts in Facebook dementia interest groups}

You're invited to participate in a research study!

My name is Angel Wang and I am a Master of Nursing student at Ryerson University in the thesis stream. I am posting to let you know about the opportunity to participate in my thesis study, titled: "Using mobile apps in caregiving: The user experiences of family caregivers of a relative living with dementia". Attached in this post is the recruitment poster.

The purpose of this study is to explore the experiences of family caregivers of a relative living with dementia who uses mobile applications (apps) in their caregiving role. This in turn can inform future mobile app developments to address caregiver needs of family caregivers and promote more effective caregiving and improved quality of life for care recipients and their family caregivers.

Participation is voluntary and is open to anyone who meet the following criteria:

- 18 to 55 years-old;

- Able to understand and provide consent in English;

- Self-identifies as an adult child family caregiver (i.e. child or grandchild) of a relative living with dementia;

- Own a mobile device (e.g. smartphone, tablet); and

- Have prior experience using mobile apps to assist in caregiving for a relative living with dementia within the last 12 months (e.g. medication reminders, "brain-training" games, calendars, social network platform).

\section{WHAT WILL YOU BE ASKED TO DO}

1. Complete a short demographic form.

2. Participate in two in-person interviews at a private location of your choosing.

3. Take pictures with your mobile device.

\section{THANK YOU}

To thank you for your participation in the study, you will be offered a $\$ 20$ gift card.

If you would like more information or are interested in participating, please contact me at he.wang@ ryerson.ca and I will be happy to answer any questions you have about the study.

This study has been approved by the Ryerson University Research Ethics Board.

Sincerely,

Angel Wang, RN, BScN, MN Student

Ryerson University

Principal Investigator 


\section{APPENDIX G}

\section{Telephone Screening Script}

Hello, my name is Angel Wang, and I am a Ryerson University graduate student. I am conducting this study as part of my Master of Nursing degree requirements. Thank you for your interest in participating in my study. Is this a good time for us to speak about the possibility of you participating in this study? (If yes, continue to 1)

1. Great. If you change your mind and want to stop the conversation or reschedule, that is not a problem.

Are you in a location that affords you some privacy?

If not: Would you like to call me back when you are? We can arrange another time to speak together.

If yes: I'd like to take a little time to briefly explain the study to you, confirm that you are an eligible participant, and explain the next steps if you are eligible. Is that okay for you? (If yes, continue to 2)

2. The purpose of this study is to explore the user experiences of family caregivers of a relative living with dementia who uses mobile applications (apps) in their caregiving role. The findings of this study can inform future mobile app developments to address caregiver needs of family caregivers and promote more effective caregiving and improved quality of life for care recipients and their family caregivers. I hope to interview 5 to 10 individuals. Participation in this study involves three components: (1) completing a short demographic form; (2) participating in two in-person interviews; and (3) taking photographs with your mobile device.

I will now confirm your eligibility for the study. To do this, I will have to ask you a couple of questions:

- Are you between the ages of 18 to 55 years-old?

- Do you self-identify as an adult child family caregiver (i.e. child or grandchild) of a relative living with dementia?

- Do you own a mobile device, such as a smartphone or tablet?

- Do you have any prior experience using mobile apps to assist in caregiving for a relative living with dementia within the last 12 months (e.g. medication reminders, "brain-training" games, calendars, social network platform)?

If the criteria are met: You meet all the eligibility criteria. Are you still interested in participating in this study?

If yes, continue to 3 .

If not: Thank you so much for expressing an interested in participating and taking the time to speak with me. All the best. Good-bye.

If the criteria are not met: Unfortunately, you are not able to participate in this study because you did meet [specific criteria]. Thank you so much for expressing an interested in participating and taking the time to speak with me. All the best. Good-bye.

3. Because you are interested in participating in this study, would you like to schedule a time for the first interview? 
If yes, schedule a time and location for the first interview.

4. Before the interview, I will send you the Consent Agreement via email which you can review. Is that okay? What is an email address that I can reach you at? After you have read the Consent Agreement, feel free to email me should you have any additional questions. I will have two copies of the Consent Agreement ready at the beginning of the interview which you can sign. One copy will be given to you for your own records. I want to remind you that you can stop participating in the study anytime without any consequences. Do you have any other questions? Thank you again for taking the time to speak with me today. I will email the Consent Agreement to you now. We will be in touch! Good-bye. 


\section{APPENDIX H}

\section{Ryerson}

University

\section{Ryerson University}

Consent Agreement

You are being invited to participate in a research study that is being conducted as part of the requirements for the completion of a Master in Nursing degree at Daphne Cockwell School of Nursing, Ryerson University.

Please read this consent form so that you understand what your participation will involve.

Before you consent to participate, please ask any questions to be sure you understand what your participation will involve.

TITLE: Using mobile apps in caregiving: The user experiences of family caregivers of persons living with dementia

\section{INVESTIGATORS:}

Principal Investigator: Angel Wang, RN, MN Student, Daphne Cockwell School of Nursing, Faculty of Community Services, Ryerson University, Toronto, Ontario.

\section{Thesis Supervisors:}

Dr. Kristine Newman, RN, CRN(C), PhD, Thesis Co-Supervisor, Daphne Cockwell School of Nursing, Faculty of Community Services, Ryerson University, Toronto, Ontario.

Dr. Lori Schindel Martin, RN, PhD, Thesis Co-Supervisor, Daphne Cockwell School of Nursing, Faculty of Community Services, Ryerson University, Toronto, Ontario.

If you have any questions or concerns about the research, please feel free to contact Angel Wang at he.wang@ryerson.ca

\section{PURPOSE OF THE STUDY:}

This study aims to explore the user experiences and perspectives of family caregivers of persons living with dementia on using mobile apps to assist with caregiving activities. For this study, mobile apps can include any apps you use on your mobile device (e.g. iPhone, iPad, Android phone, Android tablet) to assist you in providing care, including medication reminders, appointment trackers/calendars, "brain-training" games, and social media platforms.

You are eligible to participate in this study if meet the following criteria:

a) 18 to 55 years-old; 
b) Able to understand and provide consent in English;

c) Self-identifies as an adult child family caregiver (i.e. child or grandchild) of a relative living with dementia;

d) Own a mobile device (e.g. smartphone, tablet); and

e) Have prior experience using mobile apps to assist in caregiving for a relative living with dementia within the last 12 months.

\section{WHAT PARTICIPATION MEANS:}

If you choose to participate in this study, you will be asked to participate in two one-on-one interviews. The interview will be at a location that is convenient for you which is private and be free of excessive noise. The interviews will take range from 30 to 60 minutes and with your permission, will be audio-recorded. The study aims to conduct a total of 10 to 20 interviews with 5 to 10 individual participants.

\section{Overview of the Participation Process}

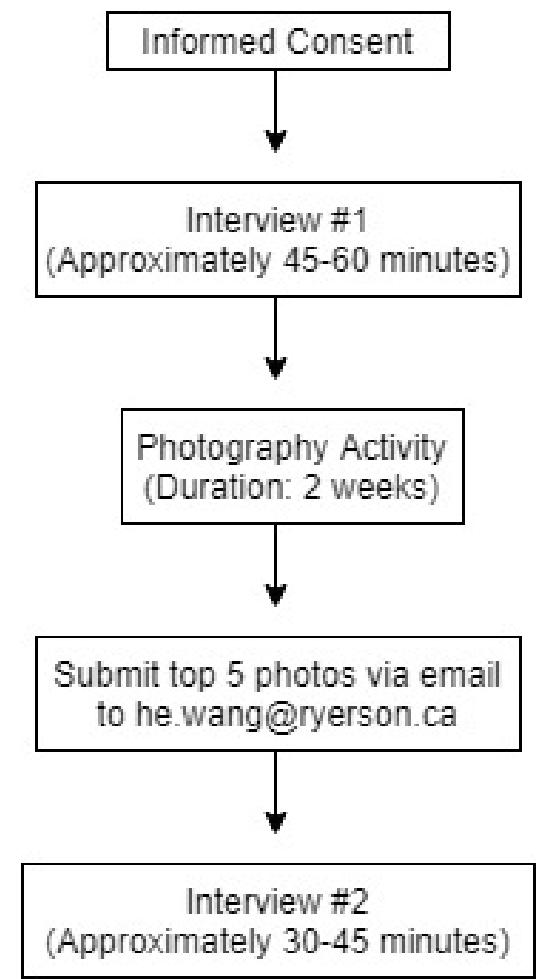

If you agree to participate in this study, you will be asked to:

1. Demographic Form: Complete a short demographic form to further understand your background.

2. Interviews: Take part in a total of two face-to-face interviews. The first interview will take approximately 45 to 60 minutes. After two weeks allocated for the photography activity, you will be asked to do a second, follow-up interview for roughly 30 to 45 minutes. 
3. Photography Activity: Take at least 5 photographs using your mobile device of images that reflect your thoughts and feelings with respect to using health-related mobile apps to support caregiving activities. The photographs can be images of inanimate objects, nature, animals, foods, etc. However, photographs are to exclude people, including you, and organizations. These photographs are to be sent to the principal investigator through email at least 3 days before the scheduled follow-up interview. The content of these photographs, and your experience of taking the photographs, will then be discussed during the second interview. They will also be used in conjunction with all of your interview data to help depict your experiences in both written language and visual representation.

4. Consent: Be asked to provide consent to the following: (a) both interviews; (b) to being audio-recorded during the two interviews; (c) to use the photographs taken by you in dissemination activities (e.g. final thesis report, publications, conference presentations).

You will not receive a copy of the research results; however, dissemination strategies will be implemented upon the completion of the study to ensure the general public, including you, will have access to the published study data (e.g. from websites of organizations, conferences, journal articles).

POTENTIAL BENEFITS: It cannot be guaranteed that your participation in the study will yield any direct benefits for you. Through participation, you may develop a greater understanding of your own experiences of using mobile applications to support caregiving activities. Further, this research can add value to the broader dialogue on family caregiving for a relative living with dementia and the potential for mobile applications to support caregiving. Findings from this research could impact public policy and draw attention to the conditions of family caregivers who care for persons living with dementia and may need technological strategies to support them in providing care. As Canada's aging population grows and continues to have complex healthcare needs, there is much potential that this research can help identify technological solutions that are inclusive and accessible and ultimately, improve outcomes for care recipients and their family caregivers.

\section{WHAT ARE THE POTENTIAL RISKS TO YOU AS A PARTICIPANT: There are} minimal risks to participating in this study. You may experience emotional discomfort or distress during the interview as a result of elucidating your experiences with caregiving. If you become distressed during the interview process, I will stop the interview and the interview will resume only if you give permission to do so. You can also decide to skip a question or end the interview at any time. You will also be given a copy of resources for family caregivers developed by Mount Sinai Hospital before the interview: Mount Sinai Hospital (March, 2017). Support for caregivers. Retrieved from https://www.mountsinai.on.ca/care/psych/patient-programs/geriatricpsychiatry/prc-dementia-resources-for-primary-care/community-resources-anddirectories/caregiver-support-services-directory 
CONFIDENTIALITY: We will respect your privacy. All information obtained during this study is strictly confidential and no information that reveals your identity will be released or published without consent unless required by law. This legal obligation includes circumstances such as suspected child or elder abuse, or expression of suicidal ideation where researchers are obliged to report to the appropriate authorities.

Interviews will first be audio recorded before being transcribed. The written interview transcript will be the focus of analysis. Once the audio file of your interview has been transferred from the recording device to a password-protected computer, your interview recording will be removed from the recording device. The audio recording device will be kept in a locked container. You will be identified only by a study number. All research data will be stored in a secure, locked location. Only members of the research team will have access to the data. This consent form and the identifying data that it contains as well as any other identifying information will be stored in a secure, locked cabinet that is separate from the study data. Following completion of the research study the data will be kept as long as required (7 years) then destroyed as required by Ryerson University's policy.

All data, including visual and interview information, that will be provided by you will be used to fulfill the purpose of this study, as well as for related presentations and potential publications. Your name or any other identifying information will not be included on the interview transcripts. Some of your comments from the interviews may be used as word-for-word quotations to present the results of the study, but you will not be directly identified as the author of those quotations. The photographs that you provide will be used in their original form for the study and in the final research report, but your identity as the photographer will remain confidential. If you express preference not to have your photographs used in the final research report (or select only a few out of the set), this will be respected.

You may withdraw your permission to use your personal information at any time by letting the study team know, and the study team will then no longer collect or share your personal information in connection with the study. However, if you withdraw your permission, you also withdraw from the study. If you withdraw from the study, you have the option to withdraw all study data by informing the contact person listed prior to data analysis, otherwise the study team will continue to use your study data that was recorded before you withdrew.

When the results of the study are published or presented, your name will not be used and no information that could identify you will be released. No identifying information will be shared when the data is disseminated.

While every effort will be made to protect the privacy of your information, absolute confidentiality cannot be guaranteed. This does not limit the duty of the researchers and others to protect your privacy. 
INCENTIVES FOR PARTICIPATION: To thank you for your participation in the study, you will receive a $\$ 10$ gift card at the end of each interview. If at any time during the interview process you decide to withdraw from the study, you will still receive this incentive for your time.

COSTS TO PARTICIPATION: There is no cost associated with participation.

VOLUNTARY PARTICIPATION AND WITHDRAWAL: Participation in this study is completely voluntary. You can choose whether to be in this study or not. If any question makes you uncomfortable, you can skip that question. You may stop participating at any time of the study. You can also request that audio-recording be stopped either temporarily or permanently at any time during the interviews. If you choose to stop participating, you may also choose to not have your data included in the study. Your choice of whether or not to participate will not influence your future relations with Ryerson University.

QUESTIONS ABOUT THE STUDY: If you have any questions about the research now, please ask. If you have questions later about the research, you may contact:

Angel Wang, RN, MN Student

he.wang@ryerson.ca

or

Kristine Newman, RN, CRN(C), PhD (Thesis Co-Supervisor)

Kristine.newman@ryerson.ca

Lori Schindel Martin, RN, PhD (Thesis Co-Supervisor)

Lori.schindelmartin@ryerson.ca

This study has been reviewed by the Ryerson University Research Ethics Board. If you have questions regarding your rights as a participant in this study, please contact:

Research Ethics Board

c/o Office of the Vice President, Research and Innovation

Ryerson University

350 Victoria Street

Toronto, ON M5B 2K3

416-979-5042

rebchair@ryerson.ca 
Ryerson

University

\section{Using mobile apps in caregiving: The user experiences of family caregivers of persons living with dementia}

\section{CONFIRMATION OF AGREEMENT:}

Your signature below indicates that you have read the information in this agreement and have had a chance to ask any questions you have about the study. Your signature also indicates that you agree to participate in the study and have been told that you can change your mind and withdraw your consent to participate at any time. You have been given a copy of this agreement. You have been told that by signing this consent agreement you are not giving up any of your legal rights.

Name of Participant (please print)

Signature of Participant

Date

\section{AUDIO-RECORDING:}

I agree to be audio-recorded for the purposes of this study. I understand how these recordings will be stored and destroyed. 
Ryerson

University

\section{Using mobile apps in caregiving: The user experiences of family caregivers of persons living with dementia}

\section{PHOTOGRAPHY ACTIVITY:}

I understand and agree that the photographs I take as part of the visual data collection step of the study will be used for presentation and dissemination purposes, and that my authorship will not be associated with these images. I understand that the photos I will be taking as part of this study will not depict people (with defining features) or organizations. I understand how these photographs will be stored and used.

Signature of Participant

Date 


\section{APPENDIX I}

\section{Demographic Data Form}

I am requesting the following information about you to help us understand your background. Remember that you may choose to skip any question you are not comfortable answering.

Participant Study ID:

1. Age:

$\square 18$ to 21 years-old

$\square 22$ to 25 years-old

26 to 29 years-old

$\square 30$ to 35 years-old

36 to 39 years old

$\square 40$ to 45 years old

46 to 49 years old

$\square 50$ to 55 years old

2. Gender:

$\square$ Female

Male

Another option

3. What city do you reside in?

4. Highest level of education:

$\square$ High school diploma

$\square$ Post-secondary (e.g. college/university)

Other:

5. My relative(s) living with dementia is/are my:

6. On which platform do/did you use to access the mobile apps?

$\square$ iPhone

$\square$ Android phone

$\square$ Personal Digital Assistant (PDA)

$\square$ Other: $\square \mathrm{iPad}$

$\square$ Android tablet

$\square$ Laptop 


\section{APPENDIX J}

\section{First Interview - Interview Guide}

\section{Dear Participant,}

My name is Angel Wang and I am a Master of Nursing student at Ryerson University. I am doing this study as part of the requirement to complete my program with the university. Thank you for agreeing to participate in this research study. I appreciate you taking the time to share your experiences with me.

The inspiration for this study came out of my own experience of being a family caregiver. After reviewing the literature, I noticed that there are limited studies incorporating the perspectives of family caregivers when developing and evaluating technological innovations to support care recipients and their family caregivers. In particular, there is currently no research on the perspectives and user experiences of family caregivers of individuals living with dementia on using mobile apps to assist in caregiving activities. As a result, I decided to develop a research study that will use the qualitative description approach to address the main research question: What are the user experiences of family caregivers using mobile apps to assist in caregiving for their loved ones living with dementia in the community?

It is anticipated that the results of the study will help better understand the perspectives and user experiences of family caregivers of individuals living with dementia on using mHealth apps to assist in caregiving activities. As such, the results may be used to inform future development and evaluations of mobile apps for family caregivers in the dementia context.

You are free to state at any time if you feel uncomfortable with a question or want to stop the interview. I will be taking brief notes during the interview. The interview should take approximately 45 to 60 minutes. The interview will be audio-recorded, do you consent to be audio-recorded? If yes: Do you have any questions before we begin with the interview?

\section{Open-ended questions:}

1. Tell me about the caregiving activities that you perform?

2. What mobile app(s) do you currently use or have used to help you with those caregiving activities?

- Do you still use that/those app(s)? How long have you used (did you use) this app for? How often do/did you use it?

- If discontinued: Why did you stop using the app(s)?

3. How do/did the mobile app(s) support you in caregiving for a loved one living with dementia?

- How do you use it to do so?

4. What prompted you to use a health-related mobile app?

- How did you 'discover' the app(s)?

$\circ$ Prompts: friend and family recommendation, healthcare professional recommendation, self-search

5. What do/did you like about this app?

- Does/did the app fulfil your needs? Why or why not?

- Is/was your app worth recommending to others?

6. How easy is/was using your app? 
- What makes/made the app information clear and understandable?

- Are/were there any parts of the app you don't use? Why?

- What app features do/did you find unintuitive?

- Who do/would/did you turn to for help using the app (prompts: family, friends, or online forum)?

7. What are some concerns you have related to using a health-related mobile app?

- Prompt: invasion of privacy, too complicated, can't find a relevant one?

- If you could redesign the app, what would you do?

8. What types of health-related mobile apps do you feel were/can be most helpful in your caregiving experience?

\section{Probing questions:}

- Could you please expand on/explain/tell me more about...?

- Can you elaborate on....? Can you tell me more about...?

- Can you explain what you meant when you said, “..."?

- I am not quite sure I understood... Could you tell more about that some more?

- I am not certain what you mean by... Could you give me some examples?

- This is what I thought I heard... Did I understand you correctly?

- So what you are saying is....?

- What are some of your reasons for thinking/feeling this way?

- Could you tell me more about your thinking on that?

- Can you give me an example?

- Would you explain that further?

- You mentioned... Could you tell me more about that?

- It sounds like you are saying, “...” Is that a correct?

\section{Closing remarks:}

- Before we close, is there anything you would like to add that was not discussed in the interview?

- Do you have any questions for me before we finish?

- Is it okay if we discuss the next steps of the study?

If yes: I will now explain the photography portion of the study.

○ Refer to Appendix K or the Photography Activity Handout.

- Is it okay if we schedule the next interview?

○ If yes: Schedule time, date, and location of the next interview.

As a token of my gratitude for your participation in this study, please accept this $\$ 10$ gift card. Thank you for your time! 


\section{APPENDIX K}

\section{Photography Activity - Information Handout}

Dear Participant,

During our first interview, I had an opportunity to engage in a conversation with you to gain a deeper understanding of your experiences of using mobile apps to assist in caregiving for a loved one living with dementia. The information you shared with me in the first interview will make up for one component of the data used for the study.

The other component of the data that will be used for the study is photographs taken by participants. I am offering you an opportunity to engage in an artistic and creative way of expressing your experiences of using mobile apps to assist in caregiving activities through the medium of photography. You can use your phone to take photographs of objects, such as stills of nature, inanimate objects (i.e. clothing), pets/animals, or foods meant to be representative of your experiences. You can also take pictures of people, but no defining features to respect their privacy such as your hand holding another person's hand. These photographs should reflect the feelings, values and ideas of your experiences of using mobile apps to assist in caregiving.

You can take as many photographs as you wish, but please only send the top five photographs that you feel reflect your experiences, feelings, values and ideas the best to my email (he.wang@ryerson.ca) at least 3 days before our second, follow-up interview. The five photographs you send will be used along with the verbal accounts you have shared to enhance and provide further insight into the meaning of your experiences. The photographs will be used in their original form as part of the final research report, and may also be used for publication or presentation purposes. You have the decision on whether to give me permission to use these photographs for the purposes of the report, publication and/or other dissemination activities. If you wish for the photographs to not be included in the final research report, publications and/or other dissemination, your decision will be respected, and your photographs will not be used.

Please be advised that the content of photographs should not depict anything illegal or be deemed sensitive in nature. Please refrain from including an individual, especially yourself, or any organizations in any of the taken photographs. Any photographs that depict people or is violating an individual or organization's privacy will not be included in the study to ensure confidentiality and anonymity.

Approximately two weeks after the initial interview, we will schedule a brief follow-up interview where we will explore the content and the meaning of the photographs. If you have any questions about this photography activity, please do not hesitate to ask me at any time during the study.

Thank you, Angel Wang, RN, BScN, MN Student

Email: he.wang@ryerson.ca 


\section{APPENDIX L}

\section{Second Interview: Interview Guide}

Dear Participant,

Thank you for meeting with me for our second, follow-up interview. I really appreciate your time. During our first interview, we discussed your experiences of using mobile apps to assist in caregiving activities. Today, I would like to focus on two things. First, I would like both of us to reflect on our initial discussion and to address any questions/clarifications we might have about the dialogue we shared. Second, we will review, reflect, and discuss the photographs that you have taken as a metaphorical representation of your experiences of using mobile apps to assist in caregiving activities.

I would like to remind you that the consent form you previously signed indicated that you can the interview at any point, and have the right not to answer any questions that might make you feel uncomfortable. I will be taking brief notes during the interview. The interview should take approximately 30 to 45 minutes. The interview will be audio-recorded, do you consent to be audio-recorded? If yes: Do you have any questions before we begin with the interview?

\section{Open-ended questions:}

1. Now that you have had some time to reflect on our initial discussion, are there any other comments that you would like to share with me today that you did not have an opportunity to bring up in our last meeting?

2. Tell me about your experience in taking photographs about your experiences of using mobile apps to assist in caregiving activities.

For each of the five pictures:

3. Can you tell me about this photograph? What is the meaning behind this photograph for you?

- What does this represent? What is the message you are trying to convey through this photograph?

- Why did you take this photograph?

4. What does this photograph mean to your caregiving experiences using mobile apps?

5. Can you tell me about what is in this photograph?

6. Where was the photograph taken?

7. Is there anything that you would want to add or change about this photograph so that it better represents your experiences of using mobile apps to assist in caregiving activities?

8. Out of the photographs that you have taken, which one, in your opinion, most closely reflects your experience of using mobile apps in caregiving activities? Please explain.

About the photography activity: Let's talk a little bit more about the photography activity itself. 
9. What were some of your thoughts and feelings as you engaged in this photography activity?

10. How did you feel completing this activity?

11. Did you encounter any challenges with this activity? If yes, please elaborate.

12. Did you gain any benefits from this activity? If yes, please elaborate.

\section{Probing questions:}

- Could you please expand on/explain/tell me more about...?

- Can you elaborate on....? Can you tell me more about...?

- Can you explain what you meant when you said, “..."?

- I am not quite sure I understood... Could you tell more about that some more?

- I am not certain what you mean by... Could you give me some examples?

- This is what I thought I heard... Did I understand you correctly?

- So what you are saying is....?

- What are some of your reasons for thinking/feeling this way?

- Could you tell me more about your thinking on that?

- Can you give me an example?

- Would you explain that further?

- You mentioned... Could you tell me more about that?

- It sounds like you are saying, “...” Is that a correct?

\section{Closing remarks:}

As our time together is coming to a close, is there anything else that you would like to share with me about your experiences of using mobile apps to support your caregiving activities? Do you have any questions for me at time?

If you do have any additional questions after today, feel free to email me at he.wang@ryerson.ca.

As a token of my gratitude for your participation in this study, please accept this $\$ 10$ gift card. Thank you for your time and participation! 


\section{APPENDIX M}

\section{List of Mobile Apps Used by Participants}

\begin{tabular}{|c|c|}
\hline Name of App & Description of App \\
\hline \multicolumn{2}{|r|}{ Social Media/Communication Apps } \\
\hline Facebook $^{\mathrm{TM}}$ & $\begin{array}{l}\text { A social media app that allows registered users to create profiles, upload } \\
\text { photos and videos, and send messages and connect with friends, family and } \\
\text { colleagues (Facebook, 2019a). }\end{array}$ \\
\hline $\begin{array}{l}\text { Facebook } \\
\text { Messenger }\end{array}$ & $\begin{array}{l}\text { A messaging app connected to Facebook that enables chat, voice and video } \\
\text { communications between Facebook users (Facebook, 2019b). }\end{array}$ \\
\hline FaceTime $^{\mathrm{TM}}$ & $\begin{array}{l}\text { An Apple app that allows users to engage in video and audio calls (Apple, } \\
\text { 2019a). }\end{array}$ \\
\hline Instagram $^{\mathrm{TM}}$ & $\begin{array}{l}\text { A photo-sharing app and social network platform that allows users to edit } \\
\text { and upload photos and short videos (Google Play, 2019a). }\end{array}$ \\
\hline Twitter $^{\mathrm{TM}}$ & $\begin{array}{l}\text { A social networking microblogging platform that allows registered users to } \\
\text { create short posts, upload photos and videos, and send messages to other } \\
\text { users (Google Play, 2019b). }\end{array}$ \\
\hline WeChat $^{\mathrm{TM}}$ & $\begin{array}{l}\text { A Chinese multi-purpose messaging, social media and mobile payment app } \\
\text { (Google Play, 2019c). }\end{array}$ \\
\hline WhatsApp ${ }^{\mathrm{TM}}$ & $\begin{array}{l}\text { An instant messaging app that allows users to exchange text, image, video } \\
\text { and audio messages as well as make video and audio calls (WhatsApp, } \\
\text { 2019). }\end{array}$ \\
\hline \multicolumn{2}{|r|}{ Productivity/Organization Apps } \\
\hline Reminder & $\begin{array}{l}\text { An app that allows users to set notifications for themselves which have } \\
\text { multiple versions on various operating systems, such as Google's Android } \\
\text { and Apple's iOS. }\end{array}$ \\
\hline $\begin{array}{l}\text { Google } \\
\text { Calendar }\end{array}$ & $\begin{array}{l}\text { A time-management and scheduling calendar service developed by Google } \\
\text { that allows users to create and edit events as well as to enable reminders for } \\
\text { events (Google Play, 2019d). }\end{array}$ \\
\hline Notes & $\begin{array}{l}\text { A note-taking app developed by Apple that allows users to create notes, } \\
\text { scan and sign documents, create checklists, add attachments, insert photos } \\
\text { and videos, as well as sketch (Apple, 2019b). Note: there are similar apps } \\
\text { for Android users. }\end{array}$ \\
\hline
\end{tabular}

Food-Related Apps 


\begin{tabular}{ll}
\hline DoorDash $^{\mathrm{TM}}$ & $\begin{array}{l}\text { An app that allows users to order food delivery from local restaurants on } \\
\text { demand across cities in the United States of America and Canada (Google } \\
\text { Play, 2019e). }\end{array}$ \\
InstaCart $^{\mathrm{TM}}$ & $\begin{array}{l}\text { An app that offers same-day grocery delivery and pick up service in the } \\
\text { United States of America and Canada (Google Play, 2019f). }\end{array}$ \\
SkipTheDishes & $\begin{array}{l}\text { TM } \\
\text { An app that allows users to order food delivery from local restaurants on } \\
\text { demand (Google Play, 2019g). }\end{array}$ \\
Uber Eats ${ }^{\mathrm{TM}}$ & $\begin{array}{l}\text { An app that allows users to order food delivery from local restaurants on } \\
\text { demand (Google Play, 2019h). }\end{array}$ \\
\hline Uber $^{\mathrm{TM}}$ & \multicolumn{1}{c}{ Transportation Apps } \\
Lyft $^{\mathrm{TM}}$ & $\begin{array}{l}\text { A ride-sharing app that allows users to request a ride from Uber drivers to } \\
\text { go to their chosen location (Google Play, 2019i). }\end{array}$ \\
& $\begin{array}{l}\text { A ride-sharing app that allows users to request a ride from Lyft drivers to } \\
\text { go to their chosen location (Google Play, 2019j). }\end{array}$
\end{tabular}

\section{Entertainment Apps}

Colouring apps A category of apps that allow users to paint mandalas, patterns, animals, florals, and thematic images.

Gardening apps A category of apps that allow users to garden virtually, such as plant seeds and care for the plants.

Netflix ${ }^{\mathrm{TM}} \quad$ A subscription-based streaming service app that offers users online streaming of a library of films and television programs (Google Play, 2019k).

Puzzle apps A category of apps that enables users to complete various puzzles.

YouTube $^{\text {TM }} \quad$ A video-sharing app that allows users to upload, view, rate and share videos (Google Play, 2019l).

\section{Miscellaneous}

\begin{tabular}{ll}
\hline Banking apps & $\begin{array}{l}\text { A category of apps that allow users to complete banking activities and } \\
\text { transactions, such as checking their balances, depositing a cheque, and } \\
\text { paying bills, with their chosen bank. }\end{array}$ \\
$\begin{array}{l}\text { Camera/Photo } \\
\text { album apps }\end{array}$ & $\begin{array}{l}\text { A category of apps that enables users to capture and store pictures and } \\
\text { videos. }\end{array}$ \\
Kijiji ${ }^{\text {TM }}$ & $\begin{array}{l}\text { A classified advertising service that allows users to post and search local } \\
\text { advertisements (Google Play, 2019m). }\end{array}$
\end{tabular}




\section{REFERENCES}

Aasbø, G., Solbrække, K. N., Kristvik, E., \& Werner, A. (2016). Between disruption and continuity: Challenges in maintaining the 'biographical we' when caring for a partner with a severe, chronic illness. Sociology of Health \& Illness, 38(5), 782-796.

Abu-Dalbouh, H., Al-Habeeb, A., Al-Kholifi, A., Al-Motiry, I., \& Al-Buhairy, M. (2015). A mobile reminder system for elderly and Alzheimer's patients. International Journal of Computer Science Issues (IJCSI), 12(5), 95.

Acharya, M. H., Gokani, T. B., Chauhan, K. N., \& Pandya, B. P. (2016, August 26-27). Android application for dementia patient. Paper presented at the International Conference on Inventive Computation Technologies (ICICT), India. doi:

10.1109/INVENTIVE.2016.7823231

Alzheimer's Association. (2013). Alzheimer's caregiving - there's an app for that! Retrieved from http://www.alzheimersblog.org/2013/08/19/alzheimers-caregiving-theres-app-that/

Alzheimer's Disease International (ADI). (2016). World Alzheimer Report 2016: Improving healthcare for people living with dementia. London, UK: Alzheimer's Disease International

Alzheimer's Disease International (ADI). (2010). World Alzheimer Report 2010: The Global Economic Impact of Dementia. London, UK: Alzheimer's Disease International Alzheimer Society of Canada. (2010). Rising Tide: The impact of dementia on Canadian society. Toronto, ON: Alzheimer Society of Canada.

Alzheimer Society of Canada. (2017a, November 8). A new way of looking at the impact of dementia in Canada. Retrieved from https://alzheimer.ca/en/cornwall/Awareness/A-newway-of-looking-at-dementia 
Alzheimer Society of Canada. (2017b, January 18). Dementia numbers in Canada. Retrieved from http://www.alzheimer.ca/en/About-dementia/What-is-dementia/Dementia-numbers

Alzheimer Society of Canada. (2017c, April 7). What is dementia? Retrieved from http://www.alzheimer.ca/en/About-dementia/What-is-dementia

Alzheimer Society of Canada (2017d, June 22). Your guide to Canada's national dementia strategy. Retrieved from https://alzheimer.ca/en/Home/Get-involved/Advocacy/Nationaldementia-strategy-guide\#what-is-a-national-dementia-strategy

Alzheimer Society of Canada. (2019, March 20). Alzheimer Society welcomes $\$ 50$ million funding for national dementia strategy. Newswire. Retrieved from https://www.newswire.ca/news-releases/alzheimer-society-welcomes-50-millionfunding-for-national-dementia-strategy-802554998.html

American Psychological Association. (2001). Publication manual of the American Psychological Association (5thed.). Washington, DC: American Psychological Association.

Anderson, K., Burford, O., \& Emmerton, L. (2016). Mobile health apps to facilitate self-care: A qualitative study of user experiences. PLoS ONE, 11(5), e0156164. doi:

10.1371/journal.pone.0156164

Andrén, S., \& Elmståhl, S. (2005). Family caregivers' subjective experiences of satisfaction in dementia care: Aspects of burden, subjective health and sense of coherence. Scandinavian Journal of Caring Sciences, 19(2), 157-168.

Andrén, S., \& Elmståhl, S. (2008). The relationship between caregiver burden, caregivers’ perceived health and their sense of coherence in caring for elders with dementia. Journal of Clinical Nursing, 17(6), 790-799.

Ansello, E. F., \& Rosenthal, C. J. (2007). Hidden costs and invisible contributions in family 
caregiving: An introduction. Canadian Journal on Aging, 26(1), 1-6.

Apple. (2019a, February 19). Use FaceTime with your iPhone, iPad, or iPod touch. Retrieved from https://support.apple.com/en-ca/HT204380

Apple. (2019b, April 22). Use Notes on your iPhone, iPad, and iPod touch. Retrieved from https://support.apple.com/en-ca/HT205773

Applebaum, A. (2015). Isolated, invisible, and in-need: There should be no "I" in caregiver. Palliative \& Supportive Care, 13(3), 415-416.

Appleton, J. V., \& King, L. (2002). Journeying from the philosophical contemplation of constructivism to the methodological pragmatics of health services research. Journal of Advanced Nursing, 40(6), 641-648.

Armstrong, P., \& Kits, O. (2001). One hundred years of caregiving. Retrieved from http://www.womenandhealthcarereform.ca/publications/care-giving-100yrs.pdf

Arntzen, C., Holthe, T., \& Jentoft, R. (2016). Tracing the successful incorporation of assistive technology into everyday life for younger people with dementia and family carers. Dementia, 15(4), 646-662.

Asghar, I., Cang, S., \& Yu, H. (2018). Usability evaluation of assistive technologies through qualitative research focusing on people with mild dementia. Computers in Human Behavior, 79, 192-201.

Ashwill, R., Mulhall, S., Johnson, D. K., \& Galvin, J. E. (2015). Caregiving experience for people with Lewy Body Dementia: Spouse versus adult child. Alzheimer's \& Dementia: The Journal of the Alzheimer's Association, 11(7), P601.

Asiedu, G. B., Carroll, K., Griffin, J. M., Hurt, R. T., \& Mundi, M. (2018). Home enteral 
nutrition: Use of photo-elicitation to capture patient and caregiver experiences. Health Science Reports, 1(8), e56.

Assistive Technology Industry Association (ATIA). (n.d.). What is AT? Retrieved from https://www.atia.org/at-resources/what-is-at/

Atchley, R.C. (2000). Social forces and aging: An introduction to social gerontology (9th edition). Belmont, CA: Wadsworth Thomson Learning.

Aubeeluck, A., \& Buchanan, H. (2006). Capturing the Huntington's disease spousal carer experience: A preliminary investigation using the 'Photovoice' method. Dementia, 5(1), 95-116. doi: $10.1177 / 1471301206059757$

Bainbridge, H. T., Cregan, C., \& Kulik, C. T. (2006). The effect of multiple roles on caregiver stress outcomes. Journal of Applied Psychology, 91(2), 490.

Balmer, C., Griffiths, F., \& Dunn, J. (2015). A review of the issues and challenges involved in using participant-produced photographs in nursing research. Journal of Advanced Nursing, 71(7), 1726-1737.

Bargas-Avila, J. A., \& Hornbæk, K. (2011, May 7-11). Old wine in new bottles or novel challenges: A critical analysis of empirical studies of user experience. Paper presented at the SIGCHI Conference on Human Factors in Computing Systems, Canada. doi: $10.1145 / 1978942.1979336$

Barnett, A. E. (2015). Adult child caregiver health trajectories and the impact of multiple roles over time. Research on Aging, 37(3), 227-252.

Bastawrous, M., Gignac, M. A., Kapral, M. K., \& Cameron, J. I. (2015). Factors that contribute to adult children caregivers' well-being: A scoping review. Health \& Social Care in the Community, 23(5), 449-466. 
Bates, E. A., McCann, J. J., Kaye, L. K., \& Taylor, J. C. (2017). “Beyond words”: A researcher's guide to using photo elicitation in psychology. Qualitative Research in Psychology, 14(4), 459-481. doi: 10.1080/14780887.2017.1359352

Bateman, D. R., Srinivas, B., Emmett, T. W., Schleyer, T. K., Holden, R. J., Hendrie, H. C., \& Callahan, C. M. (2017). Categorizing health outcomes and efficacy of mHealth apps for persons with cognitive impairment: A systematic review. Journal of Medical Internet Research, 19(8), e301.

Behind the Names. (2017a). Cassandra. Retrieved from www.behindthename.com/name/cassandra

Behind the Names. (2017b). Sophia. Retrieved from www.behindthename.com/name/sophia Behind the Names. (2018). Abigail. Retrieved from www.behindthename.com/name/abigail Behind the Names. (2019a). Sierra. Retrieved from www.behindthename.com/name/sierra Behind the Names. (2019b). Nadia. Retrieved from www.behindthename.com/name/nadia-1

Belk, R. W. (1988). Possessions and the extended self. Journal of Consumer Research, 15(2), 139-168.

Benefield, L. E., \& Beck, C. (2007). Reducing the distance in distance-caregiving by technology innovation. Clinical Interventions in Aging, 2(2), 267-272.

Bengtsson, M. (2016). How to plan and perform a qualitative study using content analysis. NursingPlus Open, 2, 8-14. doi: 10.1016/j.npls.2016.01.001

Bhattacharyya, S., Benbow, S. M., \& Collins, E. (2017). Mobile app: Living and dying well with dementia. Progress in Neurology and Psychiatry, 21(3), 4-5.

Bhattarai, P., Newton-John, T. R. O., \& Phillips, J. L. (2017). Quality and usability of arthritic 
pain self-management apps for older adults: A systematic review. Pain Medicine, 19(3), 471-484.

Bhavnani, S. P., Narula, J., \& Sengupta, P. P. (2016). Mobile technology and the digitization of healthcare. European Heart Journal, 37, 1428-1438. doi: 10.1093/eurheartj/ehv770

Bier, N., Bottari, C., Hudon, C., Joubert, S., Paquette, G., \& Macoir, J. (2013). The impact of semantic dementia on everyday actions: Evidence from an ecological study. Journal of the International Neuropsychological Society, 19(2), 162-172.

Bier, N., Paquette, G., \& Macoir, J. (2018). Smartphone for smart living: Using new technologies to cope with everyday limitations in semantic dementia. Neuropsychological Rehabilitation, 28(5), 734-754.

Billings, D. M. (2015). There's an "app” for that: Tips for preparing nurses for roles in mobile health. The Journal of Continuing Education in Nursing, 46(9), 390-391.

BinDhim, N. F., Shaman, A. M., Trevena, L., Basyouni, M. H., Pont, L. G., \& Alhawassi, T. M. (2014). Depression screening via a smartphone app: Cross-country user characteristics and feasibility. Journal of the American Medical Informatics Association, 22, 29-34. doi: 10.1136/amiajnl-2014-002840

Birkhoff, S. D., \& Smeltzer, S. C. (2017). Perceptions of smartphone user-centered mobile health tracking apps across various chronic illness populations: An integrative review. Journal of Nursing Scholarship, 49(4), 371-378.

Black, B. S., Johnston, D., Rabins, P. V., Morrison, A., Lyketsos, C., \& Samus, Q. M. (2013). Unmet needs of community-residing persons with dementia and their informal caregivers: Findings from the maximizing independence at home study. Journal of the American Geriatrics Society, 61(12), 2087-2095. 
Boots, L. M., de Vugt, M. E., van Knippenberg, R. J., Kempen, G. I. J. M., \& Verhey, F. R. J. (2013). A systematic review of internet-based supportive interventions for caregivers of patients with dementia. International Journal of Geriatric Psychiatry, 29, 331-334. doi:10.1002/gps.4016

Bowers, B. J. (1987). Intergenerational caregiving: Adult caregivers and their aging parents. Advanced Nursing Science, 9(2), 20-31.

Boydell, K. M., Gladstone, B. M., Volpe, T., Allemang, B., \& Stasiulis, E. (2012). The production and dissemination of knowledge: A scoping review of arts-based health research. Forum: Qualitative Social Research, 13(1), 1-30.

Bradshaw, C., Atkinson, S., \& Doody, O. (2017). Employing a qualitative description approach in health care research. Global Qualitative Nursing Research, 4, 1-8. doi: $10.1177 / 2333393617742282$

Braun, V., \& Clarke, V. (2006). Using thematic analysis in psychology. Qualitative Research in Psychology, 3, 77-101.

British Columbia Law Institute (BCLI). (n.d.). Chapter 2 - Family caregiving in BC. Retrieved from https://www.bcli.org/sites/default/files/Family\%20Caregiving\% 20-\%20Chapter\%202.pdf

Brodaty, H., \& Donkin, M. (2009). Family caregivers of people with dementia. Dialogues in Clinical Neuroscience, 11(2), 217-228.

Brodaty, H., Green, A., \& Koschera, A. (2003). Meta-analysis of psychosocial interventions for caregivers of people with dementia. Journal of American Geriatrics Society, 51, 657-664.

Brodaty, H., Thomson, C., Thompson, C., \& Fine, M. (2005). Why caregivers of people with 
dementia and memory loss don't use services. International Journal of Geriatric Psychiatry, 20, 537-546. doi: 10.1002/gps.1322

Brown, E. L., Ruggiano, N., Roberts, L., Hristidis, V., Whiteman, K. L., Castro, J., \& Page, T. F. (2016). CareHeroes web and Android ${ }^{\mathrm{TM}}$ apps for dementia caregivers: A feasibility study. Research in Gerontological Nursing, 9(4), 193-203.

Brown, E. L., Ruggiano, N., Li, J., Clarke, P. J., Kay, E. S., \& Hristidis, V. (2019). Smartphonebased health technologies for dementia care: Opportunities, challenges, and current practices. Journal of Applied Gerontology, 38(1), 73-91.

Buettner, L. L., Yu, F., \& Burgener, S. C. (2010). Evidence supporting technology-based interventions for people with early-stage Alzheimer's Disease. Journal of Gerontological Nursing, 36(10), 15-19. doi: 10.3928/00989134-20100831-01

Burns, K., Jayasinha, R., \& Brodaty, H. (2017). Evaluation of an electronic app developed to assist clinicians in the management of behavioral and psychological symptoms of dementia (BPSD). International Journal of Human-Computer Interaction, 1-9.

Bury, M. (1982). Chronic illness as biographical disruption. Sociology of Health \& Illness, 4(2), $167-182$.

Canada Health Infoway. (2017). One third of Canadians use mobile apps to track health: Study. Retrieved from http://www.longwoods.com/newsdetail/10966?utm_source= eLetter\&utm_campaign=c0c5fc161-Longwoods+eLetter+September+19\%2C+2017\& utm_medium=email\&utm_term=0_0f88455429-c0c5fc1616-48440873

Canadian Caregiver Coalition (CCC). (2014). A Canadian caregiver strategy: Are we making progress? Retrieved from http://www.carerscanada.ca/wp-content/uploads/2015/09/PanCanadian-Family-Caregiver-2013_WEB-PAGES-2.pdf 
Canadian Gerontological Nursing Association (CGNA). (2010). Gerontological nursing competencies and standards of practice 2010. Retrieved from https://cgna.net/uploads/CGNAStandardsOfPractice_English.pdf

Canadian Institute for Health Institute (CIHI) (2018, June 26). Almost half of unpaid caregivers of seniors with dementia experience distress: New report paints national picture of dementia and its impact on health systems and Canadians. Retrieved from https://www.cihi.ca/en/almost-half-of-unpaid-caregivers-of-seniors-with-dementiaexperience-distress

Capistrant, B. D. (2016). Caregiving for older adults and the caregivers' health: An epidemiologic review. Current Epidemiology Reports, 3, 72-80. doi: 10.1007/s40471016-0064-X

Carmeli, E. (2014). The invisibles: Unpaid caregivers of the elderly. Frontiers in Public Health, 2, 91. doi: 10.3389/fpubh.2014.00091

Catalyst. (2016). Smartphone behaviour in Canada and the implications for marketers in 2016. Retrieved from http://catalyst.ca/2016-canadian-smartphone-behaviour/

Center for Technology and Aging (CTA). (2014). The new era of connected aging: A framework for understanding technologies that support older adults in aging in place. Retrieved from http://www.techandaging.org/ConnectedAgingFramework.pdf

Chafe, R. (2017). The value of qualitative description in health services and policy research. Healthcare Policy, 22(3), 12-18.

Chappell, N. L., Dujela, C., \& Smith, A. (2014). Spouse and adult child differences in caregiving burden. Canadian Journal on Aging, 33(4), 462-472.

Chappell, N. L., \& Funk, L. M. (2011). Social support, caregiving, and aging. Canadian Journal 
on Aging, 30(3), 355-370. doi:10.1017/S0714980811000316

Chen, K., \& Chan, A. H. (2011). A review of technology acceptance by older adults. Gerontechnology, 10(1), 1-12.

Chiu, M., Wesson, V., Sadavoy, J. (2013). Improving caregiving competence, stress coping, and mental well-being in informal dementia carers. World Journal of Psychiatry, 3(3), 65-73. doi: 10.5498/wjp.v3.i3.65

Christiansen, C. H. (1999). Defining lives: Occupation as identity: An essay on competence, coherence, and the creation of meaning. The American Journal of Occupational Therapy, 53(6), 547-558.

Chua, J., \& Pachana, N. A. (2016). Use of a psychoeducational skill training DVD program to reduce stress in Chinese Australian and Singaporean dementia caregivers: A pilot study. Clinical Gerontologist, 39(1), 3-14.

Chung, P. Y. F., Ellis-Hill, C., \& Coleman, P. (2017). Supporting activity engagement by family carers at home: Maintenance of agency and personhood in dementia. International Journal of Qualitative Studies on Health and Well-being, 12(1), 1267316.

Cimperman, M., Brenčič, M. M., \& Trkman, P. (2016). Analyzing older users' home telehealth services acceptance behavior-applying an Extended UTAUT model. International Journal of Medical Informatics, 90, 22-31.

Clark, A., \& Morriss, L. (2017). The use of visual methodologies in social work research over the last decade: A narrative review and some questions for the future. Qualitative Social Work, 16(1), 29-43.

Clark-Ibáñez, M. (2004). Framing the social world with photo-elicitation interviews. American Behavioral Scientist, 47(12), 1507-1527. doi: 10.1177/0002764204266236 
Clyburn, L. D., Stones, M. J., Hadjistavropoulos, T., \& Tuokko, H. (2000). Predicting caregiver burden and depression in Alzheimer's disease. Journals of Gerontology, 55B(1), S2-S13.

Cohen, S. A., Cook, S., Kelley, L., Sando, T., \& Bell, A. E. (2015). Psychosocial factors of caregiver burden in child caregivers: Results from the new national study of caregiving. Health and Quality of Life Outcomes, 13(1), 120.

Colantonio, A., Cohen, C., \& Pon, M. (2001). Assessing support needs of caregivers of persons with dementia: Who wants what? Community Mental Health Journal, 37(3), 231-243.

Colantonio, A., Kositsky, A. J., Cohen, C., \& Vernich, L. (2001). What support do caregivers of elderly want? Results from the Canadian Study of Health and Aging. Canadian Journal of Public Health, 92(5), 376-379.

College of Nurses of Ontario (CNO). (2014a). Entry-to-practice competencies for Ontario Registered Nurses. Retrieved from https://www.cno.org/globalassets/docs/reg/41037_entrytopracitic_final.pdf

College of Nurses of Ontario (CNO). (2014b). Entry-to-practice competencies for Ontario Registered Practical Nurses. Retrieved from http://www.cno.org/globalassets/docs/reg/41042_entrypracrpn.pdf

Colorafi, K. J., \& Evans, B. (2016). Qualitative descriptive methods in health science research. Health Environments Research \& Design Journal, 9(4), 16-25. doi: $10.1177 / 1937586715614171$

Conde-Sala, J. L., Garre-Olmo, J., Turró-Garriga, O., Vilalta-Franch, J., \& López-Pousa, S. (2010a). Differential features of burden between spouse and adult-child caregivers of patients with Alzheimer's disease: An exploratory comparative design. International Journal of Nursing Studies, 47(10), 1262-1273. 
Conde-Sala, J. L., Garre-Olmo, J., Turró-Garriga, O., Vilalta-Franch, J., \& López-Pousa, S. (2010b). Quality of life of patients with Alzheimer's disease: Differential perceptions between spouse and adult child caregivers. Dementia and Geriatric Cognitive Disorders, 29(2), 97-108.

Connell, C. M., Janevic, M. R., \& Gallant, M. P. (2001). The costs of caring: Impact of dementia on family caregivers. Journal of Geriatric Psychiatry and Neurology, 179-187.

Cook, B., \& Twidle, P. (2016, October 26-27). Increasing awareness of Alzheimer's Disease through a mobile game. Paper presented at the 2016 International Conference on Interactive Technologies and Games (iTAG), United Kingdom. doi:

10.1109/iTAG.2016.16

Cooper, C., Katona, C., Orrell, M., \& Livingston, G. (2008). Coping strategies, anxiety and depression in caregivers of people with Alzheimer's disease. International Journal of Geriatric Psychiatry, 23, 929-936. doi:10.1002/gps.2007

Crespo, M., López, J., \& Zarit, S. H. (2005). Depression and anxiety in primary caregivers: A comparative study of caregivers of demented and nondemented older persons. International Journal of Geriatric Psychiatry, 20(6), 591-592. doi: 10.1002/gps. 1321

Creswell, J. W. (2013). Qualitative inquiry and research design: Choosing among five approaches. ( $\left.{ }^{\text {rd }} \mathrm{ed}.\right)$. Thousand Oaks, CA: Sage.

Creswell, J. W., \& Miller, D. L. (2000). Determining validity in qualitative inquiry. Theory into Practice, 39(3), 124-130.

Critical Appraisal Skills Programme (CASP). (2017). CASP Checklists. Retrieved from http://www.casp-uk.net/casp-tools-checklists 
Crotty, M. (1998). The foundation of social research: Meaning and perspective in the research process. Thousand Oaks, CA: Sage.

Czarnuch, S., \& Mihailidis, A. (2011). The design of intelligent in-home assistive technologies: Assessing the needs of older adults with dementia and their caregivers. Gerontechnology, 10(3), 169-182.

Davies, J., \& Gregory, D. (2007). Entering the dialogue: Marriage biographies and dementia care. Dementia, 6(4), 481-488.

Davis, B., Nies, M., Shehab, M., \& Shenk, D. (2014). Developing a pilot e-mobile app for dementia caregiver support: Lessons learned. Online Journal of Nursing Informatics, 18(1), 1-10. Retrieved from http://ojni.org/issues/?p=3095

Davis, F. D. (1989). Perceived usefulness, perceived ease of use, and user acceptance of information technology. MIS Quarterly, 13(3), 319-340.

Davis, S. W., \& Oakley-Girvan, I. (2017). Achieving value in mobile health applications for cancer survivors. Journal of Cancer Survivorship, 11, 498-504. doi: 10.1007/s11764-0170608-1

de Rotrou, J., Cantegreil, I., Faucounau, V., Wenisch, E., Chausson, C., Jegou, D., Grabar, S., \& Rigaud, A. S. (2011). Do patients diagnosed with Alzheimer's disease benefit from a psycho-educational programme for family caregivers? A randomised controlled study. International of Geriatric Psychiatry, 26, 833-842. doi: 10.1002/gps.2611

Decker, S. E., Naugle, A. E., Carter-Visscher, R., Bell, K., \& Seifert, A. (2011). Ethical issues in research on sensitive topics: Participants' experiences of distress and benefit. Journal of Empirical Research on Human Research Ethics, 6(3), 55-64.

del-Pino-Casado, R., Cardosa, M. R., López-Martínez, C., \& Orgeta, V. (2019). The association 
between subjective caregiver burden and depressive symptoms in carers of older relatives: A systematic review and meta-analysis. PLoS ONE, 14(5), e0217648.

DementiaToday. (2016). Apps to help caregivers stay organized. Retrieved from http://www.dementiatoday.com/apps-to-help-caregivers-stay-organized/

Dennison, L., Morrison, L., Conway, G., \& Yardley, L. (2013). Opportunities and challenges for smartphone applications in supporting health behavior change: Qualitative study. Journal of Medical Internet Research, 15(4), e86.

Denzin, N. K., \& Lincoln, Y. S. (2005). The Sage handbook of qualitative research (3rd ed.). Thousand Oaks, CA: Sage.

Devor, M., \& Renvall, M. (2008). An educational intervention to support caregivers of elders with dementia. American Journal of Alzheimer's Disease \& Other Dementias, 23(3), 233-241. doi: $10.1177 / 1533317508315336$

Di Mattei, V.E., Prunas, A., Novella, L., Marcone, A., Cappa, S. F., \& Sarno, L. (2008). The burden of distress in caregivers of elderly demented patients and its relationship with coping strategies. Neurological Sciences, 29, 383-389. doi:10.1007/ s10072-008-1047-6

Docking, R. E., Lane, M., \& Schofield, P. A. (2017). Usability testing of the iPhone app to improve pain assessment for older adults with cognitive impairment (prehospital setting): A qualitative study. Pain Medicine, 19(6), 1121-1131.

Dupuis, S. L., Mitchell, G. J., Jonas-Simpson, C. M., Whyte, C. P., Gillies, J. L., \& Carson, J. D. (2016). Igniting transformative change in dementia care through research-based drama. The Gerontologist, 56(6), 1042-1052.

Edmondson, A. J., Brennan, C., \& House, A. O. (2018). Using photo-elicitation to understand reasons for repeated self-harm: A qualitative study. BMC Psychiatry, 18(1), 98. 
Eloniemi-Sulkava, U., Notkola, I. L., Hentinen, M., Kivela, S. L., Sivenius, J., \& Sulkava, R. (2001). Effects of supporting community-living demented patients and their caregivers: A randomized trial. Journal of American Geriatrics Society, 49, 1282-1827.

El-Saifi, N., Moyle, W., \& Jones, C. (2018). Family caregivers' perspectives on medication adherence challenges in older people with dementia: A qualitative study. Aging \& Mental Health, 1-7.

Epstein, I., Aligato, A., Krimmel, T., \& Mihailidis, A. (2016). Older adults' and caregivers' perspectives on in-home monitoring technology. Journal of Gerontological Nursing, 42(6), 43-50. doi: http://dx.doi.org.ezproxy.lib.ryerson.ca/10.3928/0098913420160308-02

Etters, L., Goodall, D., \& Harrison, B. E. (2008). Caregiver burden among dementia patient caregivers: A review of the literature. Journal of the American Association of Nurse Practitioners, 20(8), 423-428. doi: 10.1111/j.1745-7599.2008.00342.x

Evans, D., Robertson, J., \& Candy, A. (2016). Use of photovoice with people with younger onset dementia. Dementia, 15(4), 798-813. doi: 10.1177/1471301214539955

Facebook. (2019a). Facebook. Retrieved from https://www.facebook.com/

Facebook. (2019b). Conversations come to life on Messenger. Retrieved from https://www.messenger.com/features

Family Caregiver Alliance. (2012). Digital technology for the family caregiver. Retrieved from https://www.caregiver.org/digital-technology-family-caregiver

Farran, C. J. (2002). Family caregivers: A critical resource in today's changing health-care climate. CHART, 99(4), 4-6.

Faulkner, L. (2003). Beyond the five-user assumption: Benefits of increased sample sizes in 
usability testing. Behaviour Research Methods, Instruments, and Computers, 35(3), 379383.

Fazio, S., Pace, D., Flinner, J., \& Kallmyer, B. (2018). The fundamentals of person-centered care for individuals with dementia. The Gerontologist, 58(1), S10-S19.

Filoteo, J. V., Cox, E. M., Split, M., Gross, M., Culjat, M., \& Keene, D. (2018, July 17-21). Evaluation of ReminX as a behavioral intervention for mild to moderate dementia. Paper presented at the 2018 40th Annual International Conference of the IEEE Engineering in Medicine and Biology Society (EMBC), Hawaii. doi: 10.1109/EMBC.2018.8513049

Forbes, D. A., Markle-Reid, M., Hawranik, P., Peacock, S., Kingston, D., Morgan, D., Henderson, S., Leipert, B., \& Jansen, S. L. (2008). Availability and acceptability of Canadian home and community-based services: Perspectives of family caregivers of persons with dementia. Home Health Care Services Quarterly, 27(2), 75-99. doi: $10.1080 / 01621420802022548$

Forlizzi, J., \& Battarbee, K. (2004, August 1-4). Understanding experience in interactive systems. Paper presented at the 5th Conference on Designing Interactive Systems: Processes, practices, methods, and techniques, USA. doi: 10.1145/1013115.1013152

Fusch, P. I., \& Ness, L. R. (2015). Are we there yet? Data saturation in qualitative research. The Qualitative Report, 20(9), 1408-1416.

Gagnon-Roy, M., Bourget, A., Stocco, S., Courchesne, A. C. L., Kuhne, N., \& Provencher, V. (2017). Assistive technology addressing safety issues in dementia: A scoping review. American Journal of Occupational Therapy, 71(5), 7105190020p1$7105190020 \mathrm{p} 10$.

Gallagher, D., Mhaolain, A. N., Crosby, L., Ryan, D., Lacey, L., Coen, R. F., Walsh, C., 
Coakley, D., Walsh, J. B., Cunningham, C., \& Lawlor, B. A. (2011). Self-efficacy for managing dementia may protect against burden and depression in Alzheimer's caregivers. Aging \& Mental Health, 15(6), 663-670. doi:10.1080/13607863.2011.562179

Genoe, R., \& Dupuis, S. (2013). Picturing leisure: Using photovoice to understand the experience of leisure and dementia. The Qualitative Report, 18(11), 1-21.

Genoe, M. R., \& Dupuis, S. L. (2014). The role of leisure within the dementia context. Dementia, 13(1), 33-58.

Gibson, G., Dickinson, C., Brittain, K., \& Robinson, L. (2015). The everyday use of assistive technology by people with dementia and their family carers: A qualitative study. $B M C$ Geriatrics, 15(89), 1-10. doi: 10.1186/s12877-015-0091-3

Giebel, C. M., Challis, D., \& Montaldi, D. (2015). Understanding the cognitive underpinnings of functional impairments in early dementia: A review. Aging \& Mental Health, 19(10), $859-875$.

Giebel, C. M., Challis, D. J., \& Montaldi, D. (2016). A revised interview for deterioration in daily living activities in dementia reveals the relationship between social activities and well-being. Dementia, 15(5), 1068-1081.

Giebel, C. M., \& Sutcliffe, C. (2018). Initiating activities of daily living contributes to well-being in people with dementia and their carers. International Journal of Geriatric Psychiatry, 33(1), e94-e102.

Giebel, C. M., Sutcliffe, C., \& Challis, D. (2017). Hierarchical decline of the initiative and performance of complex activities of daily living in dementia. Journal of Geriatric Psychiatry and Neurology, 30(2), 96-103.

Giebel, C. M., Sutcliffe, C., Stolt, M., Karlsson, S., Renom-Guiteras, A., Soto, M., Verbeek, H, 
Zabalegui, A., \& Challis, D. (2014). Deterioration of basic activities of daily living and their impact on quality of life across different cognitive stages of dementia: A European study. International Psychogeriatrics, 26(8), 1283-1293.

Giosa, J. L., Stolee, P., Dupuis, S. L., Mock, S. E., \& Santi, S. (2014). An examination of family caregiver experiences during care transitions of older adults. Canadian Journal on Aging, 33(2), 137-153. doi: 10.1017/S0714980814000026

Gleason, A. W. (2015). mHealth - opportunities for transforming global health care and barriers to adoption. Journal of Electronic Resources in Medical Libraries, 12(2), 114-125. doi: $10.1080 / 15424065.2015 .1035565$

Godin, K., Stapleton, J., Kirkpatrick, S. I., Hanning, R. M., \& Leatherdale, S. T. (2015). Applying systematic review search methods to the grey literature: A case study examining guidelines for school-based breakfast programs in Canada. Systematic Reviews, 4(1), 138. doi: 10.1186/s13643-015-0125-0

Godwin, K. M., Mills, W. L., Anderson, J. A., \& Kunik, M. E. (2013). Technology-driven interventions for caregivers of persons with dementia: A systematic review. American Journal of Alzheimer's Disease \& Other Dementias, 28(3), 216-222. doi: $10.1177 / 1533317513481091$

Goodwin, J., Cummins, J., Behan, L., \& O’Brien, S. M. (2016). Development of a mental health smartphone app: Perspectives of mental health service users. Journal of Mental Health, 25(5), 434-440.

Google Play. (2019a). Instagram. Retrieved from https://play.google.com/store/apps/details?id=com.instagram.android Google Play. (2019b). Twitter. Retrieved from 
https://play.google.com/store/apps/details?id=com.twitter.android

Google Play. (2019c). WeChat. Retrieved from

https://play.google.com/store/apps/details?id=com.tencent.mm

Google Play. (2019d). Google Calendar. Retrieved from

https://play.google.com/store/apps/details?id=com.google.android.calendar

Google Play. (2019e). DoorDash-Food delivery. Retrieved from

https://play.google.com/store/apps/details?id=com.dd.doordash

Google Play. (2019f). Instacart: Grocery delivery. Retrieved from

https://play.google.com/store/apps/details?id=com.instacart.client

Google Play. (2019g). SkipTheDishes - Food delivery. Retrieved from

https://play.google.com/store/apps/details?id=com.ncconsulting.skipthedishes_android

Google Play. (2019h). Uber Eats: Local food delivery. Retrieved from

https://play.google.com/store/apps/details?id=com.ubercab.eats

Google Play. (2019i). Uber. Retrieved from

https://play.google.com/store/apps/details?id=com.ubercab

Google Play. (2019j). Lyft. Retrieved from

https://play.google.com/store/apps/details?id=me.lyft.android

Google Play. (2019k). Netflix. Retrieved from

https://play.google.com/store/apps/details?id=com.netflix.mediaclient

Google Play. (20191). YouTube. Retrieved from

https://play.google.com/store/apps/details?id=com.google.android.youtube

Google Play. (2019m). Kijiji: Buy, sell and save on local deals. Retrieved from

https://play.google.com/store/apps/details?id=com.ebay.kijiji.ca 
Gottlieb, B. H., \& Wolfe, J. (2002). Coping with family caregiving to persons with dementia: A critical review. Aging \& Mental Health, 6(4), 325-342.

Government of Canada. (2010). eHealth. Retrieved from https://www.canada.ca/en/health-canada/services/health-care-system/ehealth.html

Government of Canada. (2014). Tri-council policy statement: Ethical conduct for research involving humans 2014. Retrieved from http://www.pre.ethics.gc.ca/pdf/eng/tcps22014/TCPS_2_FINAL_Web.pdf

Government of Canada. (2015a). Communications monitoring report 2015: Canada's Communications System: An overview for citizens, consumers, and creators. Retrieved from http://www.crtc.gc.ca/eng/publications/reports/policymonitoring/2015/cmr2.htm

Government of Canada. (2015b). Digital Canada 150. Retrieved from http://www.ic.gc.ca/eic/site/028.nsf/eng/home

Graneheim, U. H., \& Lundman, B. (2004). Qualitative content analysis in nursing research: Concepts, procedures and measures to achieve trustworthiness. Nurse Education Today, 24(2), 105-112.

Gray, B., Robinson, C., \& Seddon, D. (2008). Invisible children: Young carers of parents with mental health problems-the perspectives of professionals. Child and Adolescent Mental Health, 13(4), 169-172. doi: 10.1111/j.1475-3588.2007.00477.x

Guba, E. G. (1981). Criteria for assessing the trustworthiness of naturalistic inquiries. Educational Technology Research and Development, 29(2), 75-91.

Guba, E. G., \& Lincoln, Y. S. (1981). Effective evaluation. San Francisco, CA: Joey-Bass.

Guba, E. G., \& Lincoln, Y. S. (1985). Naturalistic Inquiry. Thousand Oaks, CA: Sage.

Guba, E. G., \& Lincoln, Y. S. (1998). Competing paradigms in qualitative research. In N. K. 
Denzin \& Y. S. Lincoln (Eds.), The landscape of qualitative research: Theories and issues (pp. 195-220). Thousand Oaks: Sage Publications

Hagedorn, M. (1994). Hermeneutic photography: An innovative aesthetic technique for generating data in nursing research. Advances in Nursing Science, 17(1), 44-50.

Halbach, T., Solheim, I., Ytrehus, S., \& Schulz, T. (2018). A mobile application for supporting dementia relatives: A case study. Studies in Health Technology and Informatics, 256, 839-846.

Hammer, C. S. (2011). The importance of participant demographics. American Journal of Speech-Language Pathology, 20(4), 261-261.

Han, A., Radel, J., McDowd, J. M., \& Sabata, D. (2016). Perspectives of people with dementia about meaningful activities: A synthesis. American Journal of Alzheimer's Disease \& Other Dementias, 31(2), 115-123.

Hanssen, I., \& Kuven, B. M. (2016). Moments of joy and delight: The meaning of traditional food in dementia care. Journal of Clinical Nursing, 25(5-6), 866-874.

Harper, D. (2002). Talking about pictures: A case for photo elicitation. Visual Studies, 17, 1326.

Hasselkus, B. R., \& Murray, B. J. (2007). Everyday occupation, well-being, and identity: The experience of caregivers in families with dementia. The American Journal of Occupational Therapy, 61(1), 9-20.

Health Central Healthline. (2017). New dementia app for family caregivers. Retrieved from https://www.torontocentralhealthline.ca/displayArticle.aspx $? \mathrm{id}=31774$

Hebert, R., Dubois, M. F., Wolfson, C., Chambers, L., \& Cohen, C. (2001). Factors associated 
with long-term institutionalization of older people with dementia: Data from the Canadian Study of Health and Aging. Journal of Gerontology, 56A(11), M693-M699.

Hebert, R., Levesque, L., Vezina, J., Lavoie, J. P., Ducharme, F., Gendron, C., Preville, M., Voyer, L., \& Dubois, M. F. (2003). Efficacy of a psychoeducative group program for caregivers of demented persons living at home: A randomized controlled trial. Journal of Gerontology: Social Sciences, 58B(1), S58-S67.

Hellström, I., Nolan, M., \& Lundh, U. (2007). Sustaining 'couplehood': Spouses' strategies for living positively with dementia. Dementia, 6(3), 383-409.

Hensel, B. K., Parker-Oliver, D., \& Demiris, G. (2007). Videophone communication between residents and family: A case study. Journal of the American Medical Directors Association, 8(2), 123-127.

Hilliard, M. E., Hahn, A., Ridge, A. K., Eakin, M. N., \& Riekert, K. A. (2014). User preferences and design recommendations for an mHealth app to promote cystic fibrosis selfmanagement. JMIR mHealth and uHealth, 2(4), e44.

Hirschey, J., Bane, S., Mansour, M., Sperber, J., Agboola, S., Kvedar, J., \& Jethwani, K. (2018). Evaluating the usability and usefulness of a mobile app for atrial fibrillation using qualitative methods: Exploratory pilot study. JMIR Human Factors, 5(1), e13.

Hollander, M. J., Liu, G., \& Chappell, N. L. (2009). Who cares and how much? The imputed economic contribution to the Canadian healthcare system of middle-aged and older unpaid caregivers providing care to the elderly. Healthcare Quarterly, 12(2), 42-49. doi:10.12927/hcq.2009.20660

Holthe, T., Jentoft, R., Arntzen, C., \& Thorsen, K. (2018). Benefits and burdens: Family 
caregivers' experiences of assistive technology (AT) in everyday life with persons with young-onset dementia (YOD). Disability and Rehabilitation: Assistive Technology, 13(8), 754-762.

Hong, G. R. S., \& Kim, H. (2008). Family caregiver burden by relationship to care recipient with dementia in Korea. Geriatric Nursing, 29(4), 267-274.

Hopwood, J., Walker, N., McDonagh, L., Rait, G., Walters, K., Iliffe, S., Ross, J., \& Davies, N. (2018). Internet-based interventions aimed at supporting family caregivers of people with dementia: Systematic review. Journal of Medical Internet Research, 20(6), e216.

Hsu, C. C., Wang, Y. M., Huang, C. R., Sun, F. J., Lin, J. P., Yip, P. K., \& Liu, S. I. (2017). Sustained benefit of a psycho-educational training program for dementia caregivers in Taiwan. International Journal of Gerontology, 11, 31-35. doi: http://dx.doi.org/10.1016/j.ijge.2016.05.004

Hudson, N., Culley, L., Law, C., Mitchell, H., Denny, E., \& Raine-Fenning, N. (2016). ‘We needed to change the mission statement of the marriage': Biographical disruptions, appraisals and revisions among couples living with endometriosis. Sociology of Health \& Illness, 38(5), 721-735.

Hunt, C. K. (2003). Concepts in caregiver research. Journal of Nursing Scholarship, 35(1), $27-$ 32.

Ienca, M., Fabrice, J., Elger, B., Caon, M., Pappagallo, A. S., Kressig, R. W., \& Wangmo, T. (2017). Intelligent assistive technology for Alzheimer's disease and other dementias: A systematic review. Journal of Alzheimer's Disease, 56(4), 1301-1340.

Imbeault, H., Gagnon, L., Pigot, H., Giroux, S., Marcotte, N., Cribier-Delande, P., Duval, J., Bocti, C., Lacombe, G., Fulop, T., \& Bier, N. (2018). Impact of AP@LZ in the daily life 
of three persons with Alzheimer's disease: Long-term use and further exploration of its effectiveness. Neuropsychological Rehabilitation, 28(5), 755-778. doi: https://doi.org/10.1080/09602011.2016.1172491

Imbeault, H., Langlois, F., Bocti, C., Gagnon, L., \& Bier, N. (2018). Can people with Alzheimer's disease improve their day-to-day functioning with a tablet computer? Neuropsychological Rehabilitation, 28(5), 779-796.

International Organization for Standardization (ISO). (2010). ISO 9241-210:2010. Ergonomics of human-system interaction - Part 210: Human-centred design for interactive systems. Retrieved from https://www.iso.org/obp/ui/\#iso:std:iso:9241:-210:ed-1:v1:en International Organization for Standardization (ISO). (2018). ISO 9241-11:2018(en): Ergonomics of human-system interaction - Part 11: Usability: Definitions and concepts. Retrieved from https://www.iso.org/obp/ui/\#iso:std:iso:9241:-11:ed-2:v1:en

Iribarren, S. J., Cato, K., Falzon, L., \& Stone, P. W. (2017). What is the economic evidence for mHealth? A systematic review of economic evaluations of mHealth solutions. PLoS ONE, 12(2), e0170581. doi: 10.1371/journal.pone.0170581

Joanna Briggs Institute. (2011). Reviewers' manual: 2011 edition. University of Adelaide, AUS: Joanna Briggs Institute.

Joddrell, P., \& Astell, A. J. (2017). Acto Dementia: Implementing accessibility options for dementia in existing touchscreen apps. Alzheimer's \& Dementia: The Journal of the Alzheimer's Association, 13(7), P162-P163.

Jootun, D., McGhee, G., \& Marland, G. R. (2009). Reflexivity: Promoting rigour in qualitative research. Nursing Standard, 23(23), 42-46.

Juarascio, A. S., Goldstein, S. P., Manasse, S. M., Forman, E. M., \& Butryn, M. L. (2015). 
Perceptions of the feasibility and acceptability of a smartphone application for the treatment of binge eating disorders: Qualitative feedback from a user population and clinicians. International Journal of Medical Informatics, 84(10), 808-816.

Kable, A. K., Pich, J., \& Maslin-Prothero, S. E. (2012). A structured approach to documenting a search strategy for publication: A 12 step guideline for authors. Nurse Education Today, 32(8), 878-886.

Karg, N., Graessel, E., Randzio, O., \& Pendergrass, A. (2018). Dementia as a predictor of carerelated quality of life in informal caregivers: A cross-sectional study to investigate differences in health-related outcomes between dementia and non-dementia caregivers. BMC Geriatrics, 18(1), 189.

Karr, D. (2017, September 13). How each generation has adapted to and utilizes technology. Retrieved from https://martech.zone/generation-technology/

Keller, H. H. (2016). Improving food intake in persons living with dementia. Annals of the New York Academy of Sciences, 1367(1), 3-11.

Kelly, K., \& Wolff, J. (2015). Family caregiving is finally gaining public and policy notice. Generations, 39(4), 6-10.

Kerkhof, Y. J. F., Bergsma, A., Graff, M. J. L., \& Dröes, R. M. (2017). Selecting apps for people with mild dementia: Identifying user requirements for apps enabling meaningful activities and self-management. Journal of Rehabilitation and Assistive Technologies Engineering, 4, 1-21.

Kerkhof, Y. J. F., Graff, M. J. L., Bergsma, A., de Vocht, H. H. M., \& Droes, R. (2016). Better self-management and meaningful activities thanks to tablets? Development of a personcentered program to support people with mild dementia and their carers through use of 
hand-held touch screen devices. International Psychogeriatrics, 28(11), 1917-1929. doi: $0.1017 / \mathrm{S} 1041610216001071$

Kerkhof, Y., Pelgrum-Keurhorst, M., Mangiaracina, F., Bergsma, A., Vrauwdeunt, G., Graff, M., \& Dröes, R. M. (2019). User-participatory development of FindMyApps; A tool to help people with mild dementia find supportive apps for self-management and meaningful activities. Digital Health, 5, 1-19. doi: 10.1177/2055207618822942

Khan, K. (2012, September). User experience in mobile phones by using semantic differential methodology. Paper presented at the European Conference on Information Management and Evaluation (p. 143). Academic Conferences International Limited.

Kim, B. J., Liu, L., Nakaoka, S., Jang, S., \& Browne, C. (2018). Depression among older Japanese Americans: The impact of functional (ADL \& IADL) and cognitive status. Social Work in Health Care, 57(2), 109-125.

Kim, H., Chang, M., Rose, K., \& Kim, S. (2012). Predictors of caregiver burden in caregivers of individuals with dementia. Journal of Advanced Nursing, 68(4), 846-855.

Kim, H., Sefcik, J. S., \& Bradway, C. (2016). Characteristics of qualitative descriptive studies: A systematic review. Research in Nursing \& Health, 1-20. doi: 10.1002/nur.21768

Kishchuk, O. (2018, December 31). Millennials and technology: Their digital nativity. Retrieved from https://abacusdata.ca/millennials-and-technology-their-digital-nativity/

Kitwood, T (1997). Dementia reconsidered: The person comes first. Philadelphia: Open University Press.

Klimova, B. (2017). Mobile phone apps in the management and assessment of mild cognitive impairment and/or mild-to-moderate dementia: An opinion article on recent findings. Frontiers in Human Neuroscience, 11, 461. doi: 10.3389/fnhum.2017.00461 
Klimova, B., Valis, M., \& Kuca, K. (2018). Exploring assistive technology as a potential beneficial intervention tool for people with Alzheimer's disease-a systematic review. Neuropsychiatric Disease and Treatment, 14, 3151-3158.

Kneebone, I. I., \& Martin, P. R. (2003). Coping and caregivers of people with dementia. British Journal of Health Psychology, 8, 1-17. doi:10.1348/135910703762879174

Kong, A. P. (2015). Conducting cognitive exercises for early dementia with the use of apps on iPads. Communication Disorders Quarterly, 36(2), 102-106. doi: $10.1177 / 1525740114544026$

Krauskopf, P. B., \& Golden, A. (2016). AIDSInfo HIV/AIDS Guidelines App and DementiAssist App. The Journal for Nurse Practitioners, 12(8), e373-e375.

Lai, D. W. L., \& Surood, S. (2008). Service barriers of Chinese family caregivers in Canada. Journal of Gerontological Social Work, 51(3-4), 315-336. doi:10.1080/01634370802039650

Laidlaw, R., Dixon, D., Morse, T., Beattie, T. K., Kumwenda, S., \& Mpemberera, G. (2017). Using participatory methods to design an mHealth intervention for a low income country, a case study in Chikwawa, Malawi. BMC Medical Informatics and Decision Making, 17, 98. doi: 10.1186/s12911-017-0485-6

Lapid, M. I., Atherton, P. J., Clark, M. M., Kung, S., Sloan, J. A., \& Rummans, T. A. (2015). Cancer caregiver: Perceived benefits of technology. Telemedicine and E-health, 21(11), 893-902. doi: 10.1089/tmj.2014.0117

Lapum, J. L., Liu, L., Church, K., Hume, S., Harding, B., Wang, S., Nguyen, M., Cohen, G., \& Yau, T. M. (2016). Knowledge translation capacity of arts-informed dissemination: A narrative study. Art Research International: A Transdisciplinary Journal, 1(1), 259-282. 
Law, E. L. C., Roto, V., Hassenzahl, M., Vermeeren, A. P., \& Kort, J. (2009, April 4-9). Understanding, scoping and defining user experience: A survey approach. Paper presented at the SIGCHI Conference on Human Factors in Computing Systems, USA. doi: $10.1145 / 1518701.1518813$

Lee, E. (2015). Do technology-based support groups reduce care burden among dementia caregivers? A review. Journal of Evidence-Informed Social Work, 12(5), 474-487. doi: $10.1080 / 15433714.2014 .930362$

Lee, J. A., Nguyen, H., Park, J., Tran, L., Nguyen, T., \& Huynh, Y. (2017). Usages of computers and smartphones to develop dementia care education program for Asian American family caregivers. Healthcare Informatics Research, 23(4), 338-342.

Lee, Y., \& Smith, L. (2012). Qualitative research on Korean American dementia caregivers' perception of caregiving: Heterogeneity between spouse caregivers and child caregivers. Journal of Human Behavior in the Social Environment, 22(2), 115-129.

Leff, B. (2009). Defining and disseminating the hospital-at-home model. Canadian Medical Association Journal, 180(2), 156 - 157.

Leng, F. Y., Yeo, D., George, S., \& Barr, C. (2014). Comparison of iPad applications with traditional activities using person-centred care approach: Impact on well-being for persons with dementia. Dementia, 13(2), 265-273.

Lewis, M. L., Hobday, J. V., \& Hepburn, K. W. (2010). Internet-based program for dementia caregivers. American Journal of Alzheimer's Disease \& Other Dementias, 25(8), 674679.

Lieffers, J. R. L., Vance, V. A., \& Hanning, R. M. (2014). Use of mobile device applications in 
Canadian dietetic practice. Canadian Journal of Dietetic Practice and Research, 75(1), 41-47. doi: 10.3148/75.1.2014.41

Lilly, M. B., Robinson, C. A., Holtzman, S., \& Bottorff, J. (2012). Can we move beyond burden and burnout to support the health and wellness of family caregivers to persons with dementia? Evidence from British Columbia, Canada. Health and Social Care in the Community, 20(1), 103-112. doi: 10.1111/j.1365-2524.2011.01025.x

Lim, F. S., Wallace, T., Luszcz, M. A., \& Reynolds, K. J. (2013). Usability of tablet computers by people with early-stage dementia. Gerontology, 59(2), 174-182.

Lincoln, Y. S., \& Guba, E. G. (1986). But is it rigorous? Trustworthiness and authenticity in naturalistic evaluation. New Directions for Evaluation, 1986(30), 73-84.

Lindauer, A., \& Harvath, T. A. (2014). Pre-death grief in the context of dementia caregiving: A concept analysis. Journal of Advanced Nursing, 70(10), 2196-2207.

Liu, L., Miguel Cruz, A., Rios Rincon, A., Buttar, V., Ranson, Q., \& Goertzen, D. (2015). What factors determine therapists' acceptance of new technologies for rehabilitation-a study using the Unified Theory of Acceptance and Use of Technology (UTAUT). Disability and Rehabilitation, 37(5), 447-455.

Llanque, S., Savage, L., Rosenburg, N., \& Caserta, M. (2016). Concept analysis: Alzheimer's caregiver stress. Nursing Forum, 51(1), 21-31. doi: 10.1111/nuf.12090

Lloyd, J., Patterson, T., \& Muers, J. (2016). The positive aspects of caregiving in dementia: A critical review of the qualitative literature. Dementia, 15(6), 1534-1561.

Lorenz, K., Freddolino, P. P., Comas-Herrera, A., Knapp, M., \& Damant, J. (2019). Technologybased tools and services for people with dementia and carers: Mapping technology onto the dementia care pathway. Dementia, 18(2), 725-741. 
Lwi, S. J., Ford, B. Q., Casey, J. J., Miller, B. L., \& Levenson, R. W. (2017). Poor caregiver mental health predicts mortality of patients with neurodegenerative disease. Proceedings of the National Academy of Sciences, 114(28), 7319-7324. doi:

10.1073/pnas. 1701597114

Madaleno, T. R., Moriguti, J. C., Ferriolli, E., De Carlo, M. M. R., \& Lima, N. K. (2019). Mood, lifestyle and cardiovascular risk factors among older caregivers of patients with Alzheimer's disease dementia: A case-control study. Aging Clinical and Experimental Research, 1-6.

Mahoney, D. F. (2010). An evidence-based adoption of technology model for remote monitoring of elders' daily activities. Ageing International, 36(1), 66-81. doi: 10.1007/s12126-0109073-0

Mansbach, W. E., Mace, R. A., Clark, K. M., \& Firth, I. M. (2017). Meaningful activity for longterm care residents with dementia: A comparison of activities and raters. The Gerontologist, 57(3), 461-468.

Mao, H. F., Chang, L. H., Yao, G., Chen, W. Y., \& Huang, W. N. W. (2015). Indicators of perceived useful dementia care assistive technology: Caregivers' perspectives. Geriatrics \& Gerontology International, 15(8), 1049-1057. doi: 10.1111/ggi.12398

Maresova, P., Tomsone, S., Lameski, P., Madureira, J., Mendes, A., Zdravevski, E., Chorbev, I., Trajkovik, V., Ellen, M., \& Rodil, K. (2018). Technological solutions of older people with Alzheimer's disease: Review. Current Alzheimer Research, 15, 975-983. doi: $10.2174 / 1567205015666180427124547$

Marsh, W., Shawe, J., Robinson, A., \& Leamon, J. (2016). Moving pictures: The inclusion of 
photo-elicitation into a narrative study of mothers' and midwives' experiences of babies removed at birth. Evidence Based Midwifery, 14(2), 44-48.

Martin, F., Turner, A., Wallace, L. M., \& Bradbury, N. (2013). Conceptualisation of selfmanagement intervention for people with early stage dementia. European Journal of Ageing, 10, 75-87. doi: 10.1007/s10433-012-0253-5

Mason, M. (2010). Sample size and saturation in PhD studies using qualitative interviews. Forum: Qualitative Social Research, 11(3).

Mavounza, C., Ouellet, M. C., \& Hudon, C. (2018). Caregivers' emotional distress due to neuropsychiatric symptoms of persons with amnestic mild cognitive impairment or Alzheimer's disease. Aging \& Mental Health, 1-8.

Mc Donnell, E., \& Ryan, A. (2013). Male caregiving in dementia: A review and commentary. Dementia, 12(2), 238-250.

Meiland, F. J., Bouman, A. I., Sävenstedt, S., Bentvelzen, S., Davies, R. J., Mulvenna, M. D., Nugent, C. D., Moelaert, F., Hettinga, M. E., Bengtsson, J. E., \& Dröes, R. M. (2012). Usability of a new electronic assistive device for community-dwelling persons with mild dementia. Aging \& Mental Health, 16(5), 584-591.

Meiland, F., Innes, A., Mountain, G., Robinson, L., van der Roest, H., García-Casal, J. A., Gove, D., Thyrian, J. R., Evans, S., Droes, R., Kelly, F., Kurz, A., Casey, D., Szczesniak, D., Dening, T., Craven, M. P., Span, M., Felzmann, H., Tsolaki, M., \& Franco-Martin, M. (2017). Technologies to support community-dwelling persons with dementia: A position paper on issues regarding development, usability, effectiveness and cost-effectiveness, deployment, and ethics. JMIR Rehabilitation and Assistive Technologies, 4(1), e1.

Menne, H. L., Kinney, J. M., \& Morhardt, D. J. (2002). 'Trying to continue to do as much as 
they can do' Theoretical insights regarding continuity and meaning making in the face of dementia. Dementia, 1(3), 367-382.

Meo, A. I. (2010). Picturing students' habitus: The advantages and limitations of photoelicitation interviewing in a qualitative study in the city of Buenos Aires. International Journal of Qualitative Methods, 9(2), 149-171.

Merriam-Webster. (n.d.). App. Retrieved from https://www.merriam-webster.com/dictionary/app Mickus, M. A., \& Luz, C. C. (2002). Televisits: Sustaining long distance family relationships among institutionalized elders through technology. Aging \& Mental Health, 6(4), 387396.

Middelweerd, A., van der Laan, D. M., van Stralen, M. M., Mollee, J. S., Stuij, M., te Velde, S. J., \& Brug, J. (2015). What features do Dutch university students prefer in a smartphone application for promotion of physical activity? A qualitative approach. International Journal of Behavioral Nutrition and Physical Activity, 12(1), 31.

Milani, R. V., Bober, R. M., \& Lavie, C. J. (2016). The role of technology in chronic disease care. Progress in Cardiovascular Diseases, 58, 579-583. doi: http://dx.doi.org/10.1016/j.pcad.2016.01.001

Milne, A., Guess, R., \& Russ, A. (2014). Pyscho-educational support for relatives of people with a recent diagnosis of mild to moderate dementia: An evaluation of a 'Course for Carers'. Dementia, 13(6), 768-787. doi: 10.1177/147130121348523

Milne, J., \& Oberle, K. (2005). Enhancing rigor in qualitative description: A case study. Journal of Wound Ostomy Continence Nursing, 32(6), 413-420.

Miranda-Castillo, C., Woods, B., \& Orrell, M. (2013). The needs of people with dementia living at home from user, caregiver and professional perspectives: A cross-sectional survey. BMC Health Services Research, 13(1), 43. 
Moher, D., Liberati, A., Tetzlaff, J., Altman, D. G., \& The PRISMA Group. (2009). Preferred Reporting Items for Systematic Reviews and Meta-Analyses: The PRISMA Statement. PLOS Medicine, 6(6), e1000097. doi:10.1371/journal.pmed1000097

Moon, H., \& Adams, K. B. (2013). The effectiveness of dyadic interventions for people with dementia and their caregivers. Dementia, 12(6), 821-839.

Moore, J. (2012). The benefits of mobile apps for patients and providers. British Journal of Healthcare Management, 18(9), 465-467.

Moreira, H., Oliveira, R., \& Flores, N. (2013, October 9-12). STAlz: Remotely supporting the diagnosis, tracking and rehabilitation of patients with Alzheimer's. Paper presented at the 2013 IEEE 15th International Conference on e-Health Networking, Applications \& Services (Healthcom 2013), Portugal. doi: 10.1109/HealthCom.2013.6720743

Morgan, D. G., Semchuk, K. M., Stewart, N. J., \& D’arcy, C. (2002). Rural families caring for a relative with dementia: Barriers to use of formal services. Social Science \& Medicine, 55(7), 1129-1142.

Morse, J. M. (2000). Determining sample size. Qualitative Health Research, 10(1), 3-5.

Murray, L., \& Nash, M. (2017). The challenges of participant photography: A critical reflection on methodology and ethics in two cultural contexts. Qualitative Health Research, 27(6), 923-937. doi: 10.1177/1049732316668819

National League for Nursing (NLN). (2015). A vision for the changing faculty role: Preparing students for the technological world of health care. Retrieved from http://www.nln.org/docs/default-source/about/nln-vision-series-\%28positionstatements\%29/nlnvision_8.pdf?sfvrsn=4

Neergaard, M. A., Olesen, F., Andersen, R. S., \& Sondergaard, J. (2009). Qualitative 
description-the poor cousin of health research? BMC Medical Research Methodology, 9(1), 52.

Newton, L., Dickinson, C., Gibson, G., Brittain, K., \& Robinson, L. (2016). Exploring the views of GPs, people with dementia and their carers on assistive technology: A qualitative study. BMJ Open, 6(5), e011132. doi:10.1136/bmjopen-2016-011132

Nielsen, J. (2012, June 4). How many test users in a usability study? Retrieved from http://www.nngroup.com.

Nielsen, J. \& Landauer, T.K. (1993, April 24-29). A mathematical model of the finding of usability problems. Paper presented at the ACM INTERCHI'93 Conference, The Netherlands.

Novais, T., Dauphinot, V., Krolak-Salmon, P., \& Mouchoux, C. (2017). How to explore the needs of informal caregivers of individuals with cognitive impairment in Alzheimer's disease or related diseases? A systematic review of quantitative and qualitative studies. BMC Geriatrics, 17(1), 86.

Novick, G. (2008). Is there a bias against telephone interviews in qualitative research? Research in Nursing \& Health, 31(4), 391-398.

Nygard, L. (2008). The meaning of everyday technology as experienced by people with dementia who live alone. Dementia, 7(4), 481-502. doi: 10.1177/1471301208096631

Nygard, L., \& Starkhammar, S. (2007). The use of everyday technology by people with dementia living alone: Mapping out the difficulties. Aging \& Mental Health, 11(2), 144-155. doi: $10.1080 / 13607860600844168$

Ory, M., Hoffman, P., Yee, J., Tennstedt, S., \& Schulz, R. (1999). Prevalence and impact of 
caregiving: A detailed comparison between dementia and nondementia caregivers. The Gerontologist, 39, 177-185.

Ott, C. H., Sanders, S., \& Kelber, S. T. (2007). Grief and personal growth experience of spouses and adult-child caregivers of individuals with Alzheimer's disease and related dementias. The Gerontologist, 47(6), 798-809.

Palmer, J. L. (2013). Preserving personhood of individuals with advanced dementia: Lessons from family caregivers. Geriatric Nursing, 34(3), 224-229.

Papastavrou, E., Kalokerinou, A., Papacostas, S. S., Tsangari, H., \& Sourtzi, P. (2007). Caring for a relative with dementia: Family caregiver burden. Journal of Advanced Nursing, 58(5), 446-457. doi: 10.1111/j.1365-2648.2007.04250.x

Paré, G., Bourget, C., Aguirre, M., Beaudoin, J., Boutin, S., Vachon, K., Leaver, C., Frazer, C., Ducharme, G., \& Lavoie, A. (2017). Diffusion of smart devices for health in Canada. Montreal, CA: CEFRIO and Canada Health Infoway.

Park, J., Han, S. H., Kim, H. K., Oh, S., \& Moon, H. (2013). Modeling user experience: A case study on a mobile device. International Journal of Industrial Ergonomics, 43(2), 187196. doi: 10.1016/j.ergon.2013.01.005

Parkinson, M., Carr, S. M., Rushmer, R., \& Abley, C. (2016). Investigating what works to support family carers of people with dementia: A rapid realist review. Journal of Public Health, 39(4), e290-e301.

Peacock, S., Forbes, D., Markle-Reid, M., Hawranik, P., Morgan, D., Jansen, L., Leipert, B. D., \& Henderson, S. R. (2010). The positive aspects of the caregiving journey with dementia: Using a strengths-based perspective to reveal opportunities. Journal of Applied Gerontology, 29(5), 640-659. 
Peek, S. T., Wouters, E. J., van Hoof, J., Luijkx, K. G., Boeije, H. R., \& Vrijhoef, H. J. (2014).

Factors influencing acceptance of technology for aging in place: A systematic review. International Journal of Medical Informatics, 83(4), 235-248.

Peisachovich, E., Jokel, R., Appel, L., Da Silva, C., Sinclair, D., \& Soroor, W. (2018, July 2325). SafeHome app: Design and usability for a digital application to promote physical safety to persons living with dementia. Paper presented at the 20189 th International Conference on Information, Intelligence, Systems and Applications (IISA), Greece. doi: 10.1109/IISA.2018.8633634

Penfold, J. (2014). Improving pain assessment in patients with dementia: Julie Penfold describes a high-tech tool for community nurses to use when working with confused older people. Primary Health Care, 24(6), 8-9.

Peng, W., Kanthawala, S., Yuan, S., \& Hussain, S. A. (2016). A qualitative study of user perceptions of mobile health apps. BMC Public Health, 16(1), 1158. doi:

$10.1186 / \mathrm{s} 12889-016-3808-0$

Pérès, K., Helmer, C., Amieva, H., Orgogozo, J. M., Rouch, I., Dartigues, J. F., \& BarbergerGateau, P. (2008). Natural history of decline in instrumental activities of daily living performance over the 10 years preceding the clinical diagnosis of dementia: A prospective population-based study. Journal of the American Geriatrics Society, 56(1), 37-44.

Perry, J., \& O'Connor, D. (2002). Preserving personhood: (Re)membering the spouse with dementia. Family Relations, 51(1), 55-62.

Peterson, K., Hahn, H., Lee, A. J., Madison, C. A., \& Atri, A. (2016). In the information age, do 
dementia caregivers get the information they need? Semi-structured interviews to determine informal caregivers' education needs, barriers, and preferences. $B M C$ Geriatrics, 16, 164. doi: 10.1186/s12877-016-0338-7

Phillippi, J., \& Lauderdale, J. (2017). A guide to field notes for qualitative research: Context and conversation. Qualitative Health Research, 28(3), 381-388.

Phinney, A. (2006). Family strategies for supporting involvement in meaningful activity by persons with dementia. Journal of Family Nursing, 12(1), 80-101.

Phinney, A., Chaudhury, H., \& O’Connor, D. L. (2007). Doing as much as I can do: The meaning of activity for people with dementia. Aging and Mental Health, 11(4), 384-393.

Phinney, A., Dahlke, S., \& Purves, B. (2013). Shifting patterns of everyday activity in early dementia: Experiences of men and their families. Journal of Family Nursing, 19(3), 348374.

Pinquart, M., \& Sörensen, S. (2011). Spouses, adult children, and children-in-law as caregivers of older adults: A meta-analytic comparison. Psychology and Aging, 26(1), 1-14.

Pitts, K., Pudney, K., Zachos, K., Maiden, N., Krogstie, B., Jones, S., Rose, M., MacManus, J., \& Turner, I. (2015). Using mobile devices and apps to support reflective learning about older people with dementia. Behaviour \& Information Technology, 34(6), 613-631.

Powell, J., Chiu, T., \& Eysenbach, G. (2008). A systematic review of networked technologies supporting carers of people with dementia. Journal of Telemedicine and Telecare, 14, 154-156. doi: $10.1258 / \mathrm{jtt} .2008 .003018$

Primeau, L. A. (2003). Reflections on self in qualitative research: Stories of family. American Journal of Occupational Therapy, 57(1), 9-16.

Prince, M., Bryce, R., Albanese, E., Wimo, A., Ribeiro, W., \& Ferri, C. P. (2013). The global 
prevalence of dementia: A systematic review and metaanalysis. Alzheimer's \& Dementia: The Journal of the Alzheimer's Association, 9(1), 63-75. doi: 10.1016/j.jalz.2012.11.007

Prizer, L. P., \& Zimmerman, S. (2018). Progressive support for activities of daily living for persons living with dementia. The Gerontologist, 58(1), S74-S87.

Prorok, J. C., Horgan, S., \& Seitz, D. (2013). Health care experiences of people with dementia and their caregivers: A meta-ethnographic analysis of qualitative studies. Canadian Medical Association Journal, 185(14), E669-E680. doi: 10.1503/cmaj.121795

Polit, D. F., \& Beck, C. T. (2009). International differences in nursing research, 2005-2006. Journal of Nursing Scholarship, 41, 44-53.

Polit, D. F., \& Beck, C. T. (2014). Supplement for Chapter 14: Qualitative descriptive studies. In Essentials of nursing research: Appraising evidence for nursing practice (8th ed.). Philadelphia, PA: Wolters Kluwer Health; Lippincott Willians \& Wilkins. Retrieved from http://downloads.lww.com/wolterskluwer_vitalstream_com/samplecontent/9781451176797_Polit/samples/CS_Chapter_14.pdf

Parry, R. A. (2004). The complete idiot's guide to the Talmud. Indianapolis, USA: Penguin Books USA Inc.

Quinn, C., Nelis, S. M., Martyr, A., Morris, R. G., Victor, C., \& Clare, L. (2019). Caregiver influences on 'living well' for people with dementia: Findings from the IDEAL study. Aging \& Mental Health, 1-9.

Ramanathan, N., Swendeman, D., Comulada, W. S., Estrin, D., \& Rotheram-Borus, M. J. (2013). Identifying preferences for mobile health applications for self-monitoring and selfmanagement: Focus group findings from HIV-positive persons and young mothers. International Journal of Medical Informatics, 82(4), e38-e46. 
Rasche, P., Mertens, A., Bröhl, C., Theis, S., Seinsch, T., Wille, M., Pape, H., \& Knobe, M. (2017). The "Aachen fall prevention App"-a smartphone application app for the selfassessment of elderly patients at risk for ground level falls. Patient Safety in Surgery, 11(1), 14.

Rath, T. (n.d.). You can't be anything you want to be... Retrieved from http://www.tomrath.org/can-anything-want-myth/

Rathnayake, S., Moyle, W., Jones, C., \& Calleja, P. (2018). mHealth applications as an educational and supportive resource for family carers of people with dementia: An integrative review. Dementia, O(0), 1-22. doi: 10.1177/1471301218768903

Reed, C., Belger, M., Dell'Agnello, G., Wimo, A., Argimon, J. M., Bruno, G., Dodel, R., Haro, J. M., Jones, R. W. \& Vellas, B. (2014). Caregiver burden in Alzheimer's disease: Differential associations in adult-child and spousal caregivers in the GERAS observational study. Dementia and Geriatric Cognitive Disorders, 4(1), 51-64.

Reynoldson, C., Stones, C., Allsop, M., Gardner, P., Bennett, M. I., Closs, S. J., Jones, R., \& Knapp, P. (2014). Assessing the quality and usability of smartphone apps for pain selfmanagement. Pain Medicine, 15(6), 898-909.

Rialle, V., Ollivet, C., Guigui, C., \& Hervé, C. (2008). What do family caregivers of Alzheimer's disease patients desire in smart home technologies? Methods of Information in Medicine, 47(01), 63-69.

Riley, R. G., \& Manias, E. (2004). The uses of photography in clinical nursing practice and research: A literature review. Journal of Advanced Nursing, 48(4), 397-405.

Rinaldi, P., Spazzafumo, L., Mastriforti, R., Mattioli, P., Marvardi, M., Polidori, M. C., Abate G, 
Bartorelli, L., Bonaiuto, S., Capurso, A., Cucinotta, D., Gallucci, M., Giordano, M., Martorelli, M., Masaraki, G., Nieddu, A., Pettenati, C., Putzu, P., Tammaro, V.A,, Tomassini, P.F., Vergani, C., Senin, U., \& Capurso, A. (2005). Predictors of high level of burden and distress in caregivers of demented patients: Results of an Italian multicenter study. International Journal of Geriatric Psychiatry, 20(2), 168-174.

Roland, K. P., \& Chappell, N. L. (2015). Meaningful activity for persons with dementia: Family caregiver perspectives. American Journal of Alzheimer's Disease \& Other Dementias, 30(6), 559-568.

Rolfe, G. (2006). Validity, trustworthiness and rigour: Quality and the idea of qualitative research. Journal of Advanced Nursing, 53(3), 304-310. doi:10.1111/j.13652648.2006.03727.x

Rosenberg, L., Kottorp, A., \& Nygård, L. (2012). Readiness for technology use with people with dementia: The perspectives of significant others. Journal of Applied Gerontology, 31(4), $510-530$.

Rouse, M. (2005, September). Connection. Retrieved from https://searchnetworking.techtarget.com/definition/connection

Rozario, P. A., Morrow-Howell, N., \& Hinterlong, J. E. (2004). Role enhancement or role strain: Assessing the impact of multiple productive roles on older caregiver wellbeing. Research on Aging, 26(4), 413-428.

Ruggiano, N., Brown, E. L., Li, J., \& Scaccianoce, M. (2018). Rural dementia caregivers and technology: What is the evidence? Research in Gerontological Nursing, 11(4), 216-224.

Ruggiano, N., Brown, E. L., Shaw, S., Geldmacher, D., Clarke, P., Hristidis, V., \& Bertram, J. 
(2019). The potential of information technology to navigate caregiving systems:

Perspectives from dementia caregivers. Journal of Gerontological Social Work, 62(4), 432-450.

Sandelowski, M. (1986). The problem of rigor in qualitative research. Advances in Nursing Science, 8(3), 27-37.

Sandelowski, M. (1993). Rigor or rigor mortis: The problem of rigor in qualitative research revisited. Advances in Nursing Science, 16(2), 1-8.

Sandelowski, M. (1995). Sample size in qualitative research. Research in Nursing \& Health, 18(2), 179-183. doi:10.1002/nur.4770180211

Sandelowski, M. (2000). Whatever happened to qualitative description? Research in Nursing \& Health, 23(4), 334-340. doi: 10.1002/1098-240X(200008)23:4<334::AIDNUR9>3.0.CO;2-G

Sandelowski, M. (2010). What's in a name? qualitative description revisited. Research in Nursing \& Health, 33(1), 77-84. doi: 10.1002/nur.20362

Sandhu, A., Ives, J., Birchwood, M., \& Upthegrove, R. (2013). The subjective experience and phenomenology of depression following first episode psychosis: A qualitative study using photo-elicitation. Journal of Affective Disorders, 149(1-3), 166-174.

Sanjari, M., Bahramnezhad, F., Fomani, F. K., Shoghi, M., \& Cheraghi, M. A. (2014). Ethical challenges of researchers in qualitative studies: The necessity to develop a specific guideline. Journal of Medical Ethics and History of Medicine, 7(14), 1-6.

Sarkar, U., Gourley, G. I., Lyles, C. R., Tieu, L., Clarity, C., Newmark, L., Singh, K., \& Bates, D. W. (2016). Usability of commercially available mobile applications for diverse patients. Journal of General Internal Medicine, 31(12), 1417-1426. 
Sasaki, H. (2016, May 16-20). A smartphone app provides preventive care for the elderly with dementia. Paper presented at the 2016 IEEE 32nd International Conference on Data Engineering Workshops (ICDEW), Finland. doi: 10.1109/ICDEW.2016.7495615

Sävenstedt, S., Brulin, C., \& Sandman, P. O. (2003). Family members' narrated experiences of communicating via video-phone with patients with dementia staying at a nursing home. Journal of Telemedicine and Telecare, 9(4), 216-220.

Savita, K. S., Marrima, J. J. D. A., Muniandy, M., Abidin, A. I. Z., \& Taib, S. M. (2018, November 21-22). Help me! MyDem application for early stage dementia patients. Paper presented at the 2018 IEEE Conference on e-Learning, e-Management and e-Services (IC3e), Malaysia. doi: 10.1109/IC3e.2018.8632632

Schumacher, K., Beck, C. A., \& Marren, J. M. (2006). Family caregivers: Caring for older adults, working with their families. The American Journal of Nursing, 106(8), 40-49.

Schoenmakers, B., Buntinx, F., \& Delepeleire, J. (2010). Factors determining the impact of caregiving on caregivers of elderly patients with dementia. A systematic literature review. Maturitas, 66(2), 191-200.

Scott, L. D. (2001). Technological caregiving: A qualitative perspective. Home Health Care Management and Practice, 13(3), 227-235.

Semple, J. L., \& Armstrong, K. A. (2017). Mobile applications for postoperative monitoring after discharge. Canadian Medical Association Journal, 189, E22-34. doi: 10.1503/cmaj.160195

Senate of Canada. (2016). Dementia in Canada: A national strategy for dementia-friendly communities. Retrieved from https://sencanada.ca/content/sen/committee/421/SOCI/ Reports/SOCI_COM_ExecutiveSummary_dementia_2016-11-14_E_Final.pdf 
Shell, L. (2014). Photo-elicitation with autodriving in research with individuals with mild to moderate Alzheimer's disease: Advantages and challenges. International Journal of Qualitative Methods, 13(1), 170-184.

Shim, B., Barroso, J., \& Davis, L. L. (2012). A comparative qualitative analysis of stories of spousal caregivers of people with dementia: Negative, ambivalent, and positive experiences. International Journal of Nursing Studies, 49(2), 220-229.

Short, J., Williams, E., \& Christie, B. (1976). The social psychology of telecommunications. London: John Wiley \& Sons.

Shreve, J., Baier, R., Epstein-Lubow, G., \& Gardner, R. L. (2016). Dementia caregivers’ technology preferences: Design insights from qualitative interviews. Gerontechnology, 14(2), 89-96. doi: 10.4017/gt.2016.14.2.004.00

Silva, B. M., Rodrigues, J. J., de la Torre Díez, I., López-Coronado, M., \& Saleem, K. (2015). Mobile-health: A review of current state in 2015. Journal of Biomedical Informatics, 56, 265-272.

Siminerio, L. M. (2010). The role of technology and the chronic care model. Journal of Diabetes Science and Technology, 4(2), 471-475.

Sindi, S., Calov, E., Fokkens, J., Ngandu, T., Soininen, H., Tuomilehto, J., \& Kivipelto, M. (2015). The CAIDE Dementia Risk Score App: The development of an evidence-based mobile application to predict the risk of dementia. Alzheimer's \& Dementia: Diagnosis, Assessment \& Disease Monitoring, 1(3), 328-333.

Smith, S. K., \& Mountain, G. A. (2012). New forms of information and communication technology (ICT) and the potential to facilitate social and leisure activity for people living with dementia. International Journal of Computers in Healthcare, 1(4), 332-345. 
Solanas, A., Martinez-Balleste, A., Perez-Martinez, P. A., de la Pena, A. F., \& Ramos, J. (2013). m-Carer: Privacy-aware monitoring for people with mild cognitive impairment and dementia. IEEE Journal on Selected Areas in Communications, 31(9), 19-27.

Span, M., Hettinga, M., Vernooij-Dassen, M., Eefsting, J., \& Smits, C. (2013). Involving people with dementia in the development of supportive IT applications: A systematic review. Ageing Research Reviews, 12(2), 535-551.

Spitznagel, M. B., Tremont, G., Davis, J. D., \& Foster, S. M. (2006). Psychosocial predictors of dementia caregiver desire to institutionalize: Caregiver, care recipient, and family relationship factors. Journal of Geriatric Psychiatry and Neurology, 19(1), 16-20.

Sposaro, F., Danielson, J., \& Tyson, G. (2010, August). iWander: An Android application for dementia patients. Paper presented at the 2010 Annual International Conference of the IEEE Engineering in Medicine and Biology Society (EMBC), Argentina. doi: 10.1109/IEMBS.2010.5627669

Spradley, J. P. (1979). The ethonographic interview. New York, USA: Holt, Rinehart and Winston.

Springate, B. A., \& Tremont, G. (2014). Dimensions of caregiver burden in dementia: Impact of demographic, mood, and care recipient variables. The American Journal of Geriatric Psychiatry, 22(3), 294-300.

Stall, N. M., Kim, S. J., Hardacre, K. A., Shah, P. S., Straus, S. E., Bronskill, S. E., Lix, L. M., Bell, C. M., \& Rochon, P. A. (2019). Association of informal caregiver distress with health outcomes of community-dwelling dementia care recipients: A systematic review. Journal of the American Geriatrics Society, 67(3), 609-617.

Statistics Canada. (2012, May). Generations in Canada. Retrieved from 
https://www12.statcan.gc.ca/census-recensement/2011/as-sa/98-311-x/98-311x2011003_2-eng.cfm

Statistics Canada. (2018, July 3). Linguistic characteristics of Canadians. Retrieved from https://www12.statcan.gc.ca/census-recensement/2011/as-sa/98-314-x/98-314-x2011001eng.cfm

Steinhubl, S. R., Muse, E. D., \& Topol, E. J. (2013). Can mobile health technologies transform health care? Journal of the American Medical Association, 310(22), 23-24. doi: 10.1001/jama.2013.281078

Stewart, M., Barnfather, A., Neufeld, A., Warren, S., Letourneau, N., \& Liu, L. (2006). Accessible support for family caregivers of seniors with chronic conditions: From isolation to inclusion. Canadian Journal on Aging, 25(2), 179-192.

Streubert, H. J., \& Carpenter, D. R. (2013). Qualitative research in nursing: Advancing the humanistic imperative. (6 $6^{\text {th }}$ ed.). Philadelphia: Lippincott, Williams \& Wilkins.

Suijkerbuijk, S., Nap, H. H., Cornelisse, L., IJsselsteijn, W. A., de Kort, Y. A., \& Minkman, M. (2019). Active involvement of people with dementia: A systematic review of studies developing supportive technologies. Journal of Alzheimer's Disease, 69(4), 1041-1065.

Sullivan-Bolyai, S., Bova, C., \& Harper, D. (2005). Developing and refining interventions in persons with health disparities: The use of qualitative description. Nursing Outlook, 53(3), 127-133. doi: 10.1016/j.outlook.2005.03.005

Sun, F. (2014). Caregiving stress and coping: A thematic analysis of Chinese family caregivers of persons with dementia. Dementia, 13(6), 803-818.

Suri, H. (2011). Purposeful sampling in qualitative research synthesis. Qualitative Research Journal, 11(2), 63-75. doi: 10.3316/QRJ1102063 
Szabo, S. M., Whitlatch, C. J., Orsulic-Jeras, S., \& Johnson, J. D. (2016). Recruitment challenges and strategies: Lessons learned from an early-stage dyadic intervention (innovation practice). Dementia, 1-6. doi: 10.1177/1471301216659608

Tam-Tham, H., Nettel-Aguirre, A., Silvius, J., Dalziel, W., Garcia, L., Molnar, F., \& Drummond, N. (2016). Provision of dementia-related services in Canada: A comparative study. BMC Health Services Research, 16, 184. doi: 10.1186/s12913-016-1435-1

Tamim, S. R., \& Grant, M. M. (2016). Exploring how health professionals create eHealth and mHealth education interventions. Educational Technology Research and Development, 64, 1053-1081. doi: 10.1007/s11423-016-9447-4

Tao, H., McRoy, S., Kovach, C. R., \& Wang, L. (2016). Performance and usability of tablet computers by family caregivers in the United States and China. Research in Gerontological Nursing, 9(4), 183-192. doi: 10.3928/19404921-20160229-01

Thordardottir, B., Malmgren Fänge, A., Lethin, C., Rodriguez Gatta, D., \& Chiatti, C. (2019). Acceptance and use of innovative assistive technologies among people with cognitive impairment and their caregivers: A systematic review. BioMed Research International, 2019. doi: https://doi.org/10.1155/2019/9196729

Thorne, S., Kirkham, S.R., \& MacDonald-Emes, J. (1997). Interpretive description: A noncategorical qualitative alternative for developing nursing knowledge. Research in Nursing \& Health, 20, 169-177.

Tobin, G. A., \& Begley, C. M. (2004). Methodological rigour within a qualitative framework. Journal of Advanced Nursing, 48(4), 388-396. doi:10.1111/j.1365-2648.2004.03207.x Topo, P. (2009). Technology studies to meet the needs of people with dementia and their 
caregivers. Journal of Applied Gerontology, 28(1), 5-37. doi:

$10.1177 / 0733464808324019$

Torp, S., Hanson, E., Hauge, S., Ulstein, I., \& Magnusson, L. (2008). A pilot study how information and communication technology may contribute to health promotion among elderly spousal carers in Norway. Health and Social Care in the Community, 16(1), 7585. doi: $10.1111 / \mathrm{j} .1365-2524.2007 .00725 . \mathrm{x}$

Torre, D. \& Murphy, J. (2015) A different lens: Changing perspectives using photoelicitation interviews. Education Policy Analysis Archives, 23(111), n111. doi: http://dx.doi.org/10.14507/epaa.v23.2051

Tremont, G., Davis, J. D., Bishop, D. S., \& Fortinsky, R. H. (2008). Telephone-delivered psychosocial intervention reduces burden in dementia caregivers. Dementia, 7(4), 503520.

Tsai, H. H., \& Tsai, Y. F. (2010). Older nursing home residents' experiences with videoconferencing to communicate with family members. Journal of Clinical Nursing, 19(11-12), 1538-1543.

Tuckett, A. G. (2005). Part II. Rigour in qualitative research: complexities and solutions. Nurse Researcher, 13(1), 29-42.

Turcotte, M. (2013). Family caregiving: What are the consequences? Retrieved from https://www.statcan.gc.ca/pub/75-006-x/2013001/article/11858-eng.htm

Tyack, C., \& Camic, P. M. (2017). Touchscreen interventions and the well-being of people with dementia and caregivers: A systematic review. International Psychogeriatrics, 1-20. doi: $10.1017 /$ S1041610217000667

University Health Network (UHN). (2017). Health \& wellness mobile apps. Retrieved from 
http://www.uhn.ca/PatientsFamilies/Health_Information/Patient_Family_Education/Libra ries/Documents/Libraries_Mobile_Health_List.pdf

Vaismoradi, M., Turunen, H., \& Bondas, T. (2013). Content analysis and thematic analysis:

Implications for conducting a qualitative descriptive study. Nursing and Health Sciences, 15, 398-405. doi: 10.1111/nhs. 12048

Vandepitte, S., Putman, K., Van Den Noortgate, N., Verhaeghe, S., Mormont, E., Van Wilder, L., De Smedt., D., \& Annemans, L. (2018). Factors associated with the caregivers' desire to institutionalize persons with dementia: A cross-sectional study. Dementia and Geriatric Cognitive Disorders, 46(5-6), 298-309.

Van der Roest, H. G., Meiland, F. J., Comijs, H. C., Derksen, E., Jansen, A. P., Van Hout, H. P., Jonker, C., \& Dröes, R. M. (2009). What do community-dwelling people with dementia need? A survey of those who are known to care and welfare services. International Psychogeriatrics, 21(5), 949-965.

Van Mierlo, L. D., Meiland, F. J. M., Van der Roest, H. G., \& Droes, R. M. (2011). Personalised caregiver support: Effectiveness of psychosocial interventions in subgroups of caregivers of people with dementia. International Journal of Geriatric Psychiatry, 27, 1-14. doi: $10.1002 / g p s .2694$

Varshney, S., Varshney, J., Varshney, U., \& Varshney, S. (2016). AlzhaTV: A smart phone app for managing depression, anxiety, and agitation in nursing home dementia patients. The American Journal of Geriatric Psychiatry, 24(3), S148-S149.

Venkatesh, V., Morris, M. G., Davis, G. B., \& Davis, F. D. (2003). User acceptance of information technology: Toward a unified view. MIS Quarterly, 425-478.

Vernooij-Dassen, M. J. F. J. (2007). Meaningful activities for people with dementia. Aging \& 
Mental Health, 11(4), 359-360.

Virzi, R.A. (1992). Redefining the test phase of usability evaluation: How many subjects is enough? Human Factors, 34, 457-468.

Wang, J., Yao, N., Shen, M., Zhang, X., Wang, Y., Liu, Y., Geng, Z., \& Yuan, C. (2016). Supporting caregivers of children with acute lymphoblastic leukemia via a smartphone app: A pilot study of usability and effectiveness. CIN: Computers, Informatics, Nursing, 34(11), 520-527.

Wang, Y. N., Shyu, Y. I. L., Chen, M. C., \& Yang, P. S. (2011). Reconciling work and family caregiving among adult-child family caregivers of older people with dementia: Effects on role strain and depressive symptoms. Journal of Advanced Nursing, 67(4), 829-840.

Ward, G., Walker-Clarke, A., \& Holliday, N. (2017). Evaluation of a web-based app to assist home-hazard modification in falls prevention. British Journal of Occupational Therapy, 80(12), 735-744.

Ward-Griffin, C. (2012). Supportive care to family caregivers is not supportive enough: Moving towards an equitable approach to dementia home care. Neurodegenerative Disease Management, 2(2), 173-181. doi: 10.2217/nmt.11.83

Ward-Griffin, C., Hall, J., DeForge, R., St-Amant, O., McWilliam, C., Oudshoorn, A., Forbes, D., \& Klosek, M. (2012). Dementia home care resources: How are we managing? Journal of Aging Research, 2012. doi: 10.1155/2012/590724

Warner, E., Johnson, L., \& Andrews, F. (2016). Exploring the suburban ideal: Residents' experiences of photo elicitation interviewing (PEI). International Journal of Qualitative Methods, 15(1), 1-9.

Washington, K. T., Meadows, S. E., Elliot, S. G., \& Koopman, R. J. (2011). Information needs 
of informal caregivers of older adults with chronic health conditions. Patient Education and Counseling, 83, 37-44. doi: 10.1016/j.pec.2010.04.017

WhatsApp. (2019). Features. Retrieved from https://www.whatsapp.com/features/

Whicher, D. M., Miller, J. E., Dunham, K. M., \& Joffe, S. (2015). Gatekeepers for pragmatic clinical trials. Clinical Trials, 12(5), 442-448.

Whitebird, R. R., Kreitzer, M. J., Lewis, B. A., Hanson, L. R., Crain, L., Enstad, C. J., \& Mehta, A. (2011). Recruiting and retaining family caregivers to a randomized controlled trial on mindfulness-based stress reduction. Contemporary Clinical Trials, 32(5), 654-661. doi: 10.1016/j.cct.2011.05.002

Wiersma, E. C. (2011). Using photovoice with people with early-stage Alzheimer's disease: A discussion of methodology. Dementia, 10, 203-216. doi:10.1177/1471301211398990

Willis, D. G., Sullivan-Bolyai, S., Knafl, K., \& Zichi-Cohen, M. (2016). Distinguishing features and similarities between descriptive phenomenological and qualitative description research. Western Journal of Nursing Research, 38, 1185-1204. doi:

$10.1177 / 0193945916645499$

Wong, S. S., George, T. J., Godfrey, M., Le, J., \& Pereira, D. B. (2019). Using photography to explore psychological distress in patients with pancreatic cancer and their caregivers: A qualitative study. Supportive Care in Cancer, 27(1), 321-328.

World Health Organization (WHO). (2011). mHealth: New horizons for health through mobile technologies. Geneva, Switzerland: WHO.

World Health Organization (WHO). (2017, May 29). World Health Assembly endorses global action plan on dementia. Retrieved from http://www.who.int/mental_health/neurology/dementia/en/ 
Xu, W., \& Liu, Y. (2015). mHealthApps: A repository and database of mobile health apps. JMIR mHealth and uHealth, 3(1), e28.

Yap, L. K. P., Seow, C. C. D., Henderson, L. M., \& Goh, Y. N. J. (2005). Family caregivers and caregiving in dementia. Reviews in Clinical Gerontology, 15(3-4), 263-271. doi:

$10.1017 / \mathrm{S} 0959259806001900$

Yasuda, K., Kuwahara, N., Kuwabara, K., Morimoto, K., \& Tetsutani, N. (2013). Daily assistance for individuals with dementia via videophone. American Journal of Alzheimer's Disease \& Other Dementias, 28(5), 508-516.

Young Dementia UK. (n.d.). Helpful technology. Retrieved from https://www.youngdementiauk.org/helpful-technology

Yu, S. F. D., Cheng, S. T., \& Wang, J. (2018). Unravelling positive aspects of caregiving in dementia: An integrative review of research literature. International Journal of Nursing Studies, 79, 1-26.

Zachos, K., Maiden, N., Pitts, K., Jones, S., Turner, I., Rose, M., Pudney, K., \& MacManus, J. (2013, June 17-20). A software app to support creativity in dementia care. Paper presented at the 9th ACM Conference on Creativity \& Cognition, Australia. doi: $10.1145 / 2466627.2466637$

Zapata, B. C., Fernandez-Aleman, J. L., Idri, A., \& Toval, A. (2015). Empirical students on usability of mHealth apps: A systematic literature review. Journal of Medical Systems, 39(1) 1-19. doi: 10.1007/s10916-014-0182-2

Zelinski, E. M., Zak, D. K., \& Grossman, M. (2017). Mobile apps for caregivers generally address some but not all forms of evidence-based support. Alzheimer's \& Dementia: The Journal of the Alzheimer's Association, 13(7), P162. 
Zhao, Y., Feng, H., Hu, M., Hu, H., Li, H., Ning, H., Chen, H., Liao, L., \& Peng, L. (2019). Web-based interventions to improve mental health in home caregivers of people with dementia: Meta-analysis. Journal of Medical Internet Research, 21(5), e13415. doi: $10.2196 / 13415$

Zimmermann, M. (2013). 'Journeys' in the life-writing of adult-child dementia caregivers. Journal of Medical Humanities, 34(3), 385-397.

Zorluoglu, G., Kamasak, M. E., Tavacioglu, L., \& Ozanar, P. O. (2015). A mobile application for cognitive screening of dementia. Computer Methods and Programs in Biomedicine, 118(2), 252-262. 